NUREG/CR-2449

PNL-4122

\title{
Survey of Radionuclide Distributions Resulting from the Church Rock, New Mexico, Uranium Mill Tailings Pond Dam Failure
}

Prepared by W. C. Weimer, R. R. Kinnison, J. H. Reeves

Pacific Northwest Laboratory

Operated by

Battelle Memorial Institute

Prepared for

U.S. Nuclear Regulatory

Commission 


\section{NOTICE}

This report was prepared as an account of work sponsored by an agency of the United States Government. Neither the United States Government nor any agency thereof, or any of their employees, makes any warranty, expressed or implied, or assumes any legal liability or responsibility for any third party's use, or the results of such use, of any information. apparatus product or process disclosed in this report. or represents that its use by such third party would not infringe privately owned rights.

\section{Available from}

GPO Sales Program

Division of Technical Information and Document Control

U. S. Nuclear Regulatory Commission Washington, D. C. 20555

Printed copy price: $\$ 6.50$

$$
\text { and }
$$

National Technical Information Service

Springfield, Virginia 22161 


\section{Survey of Radionuclide Distributions Resulting from the Church Rock, New Mexico, Uranium Mill Tailings Pond Dam Failure}

Manuscript Completed: November 1981

Date Published: December 1981

Prepared by

W. C. Weimer, R. R. Kinnison, J. H. Reeves

Pacific Northwest Laboratory

Richland, WA 99352

\section{Prepared for}

Division of Waste Management

Office of Nuclear Material Safety and Safeguards

U.S. Nuclear Regulatory Commission

Washington, D.C. 20555

NRC FIN B2214 



\section{ACKNOWLEDGEMENTS}

This investigation required that consicierable time be spent at a field site, the United Nuclear Corporation (UNC) Church Rock, New Mexico, uranium mi11. Cooperative interaction between personnel from Pacific Northwest Laboratory (PNL), the Nuclear Regulatory Commission, the New Mexico EnvironmentaT Improvement Division (NMEID), and the United NuClear Corporation was required. This necessary cooperation was generously furnisheo in all cases and the provision of easy site access and communication facilitated the research. Several personnel were especially helpful and cooperative. The Project Manager for the Nuclear Regulatory Commission, Gregory Eadie, nas been extremely helpful and supportive and has functioned as an intermediary between al! of the organizations involved throughout the course of this stuay. The two persons from the New Mexico Environmental Improvement Division who have been the most involved in our research are Drs. Thomas Buhl and Jere Millard. A large portion of the work was carried out at the Uniteo Nuclear Corporation Church Rock Mill site and used UNC property and personnel. Harmonious interactions between UNC and PNL personnel allowed our research to progress smoothly. The UNC personnel to whom we are the most grateful are Todd Miller, Gus Swanquist, Robert Miller, and kee Benally. We thank the personnel from each of these organizations for their very helpfut assistance in this research. 



\section{ABSTRACT}

An intensive site survey and on-site analysis program were conducted to evaluate the distribution of four radionuclioes in the general vicinity of Gallup, New Mexico, subsequent to the accidental breach of a uranium mill tailings pond dam and the release of a large quantity of tailings pond materials. The objective of this work was to determine the distribution and concentration levels of ${ }^{210} \mathrm{~Pb},{ }^{226} \mathrm{Ra},{ }^{230} \mathrm{Th}$, and ${ }^{238} \mathrm{U}$ in the arroyo that is imrnediately adjacent to the uranium tailings pond (pipeline arroyo) and in the Rio Puerco arroyo into which the pipeline arroyo drains. An intensive survey between the United Nuclear Corporation (UNC) Church Rock Mill site and the New MexicoArizona state border was performed. Sampling locations were established at approximately 500-ft intervals along the arroyo. During the weeks of September 24 through 0ctober 5, 1979, a series of samples was collected from alternate sampling locations along the arroyo. The purpose of this collection of samples and their subsequent analysis was to provide an immediate evaluation of the extent and the levels of radioactive contamination. The data obtained from this extensive survey were then compared to action levels which had been proposed by the Nuclear Regulatory Commission and were adapted by the New Mexico

Environmental Improvement Division (NMEID) for ${ }^{230}$ Th and ${ }^{226}$ Ra concentrations that would require site cleanup.

The Pacific Northwest Laboratory/Nuclear Regulatory Commission mobile laboratory van was on-site at the UNC Church Rock Mill from September 22, 1979, through December 13, 1979, and was manned by one or more PNL personnel for all but four weeks of this time period. Approximately 1200 samples associated with the Rio Puerco survey were analyzed in the laboratory. An additional 1200 samples related to the Rio Puerco cleanup operations which the United Nuclear Corporation was conducting were analyzed on-site in the mobile laboratory. The purpose of these analyses was to determine the effectiveness of the cleanup operations that were ongoing and to evaluate what additional cleanup would be required. This on-site analysis of radioactive contamination constituted the principal task of this project, with the identification of those portions of the arroyo exceeding the NMEID proposed cleanup criteria being the 
major output. Additional tasks included an evaluation of the initial soil sampling scheme (letter from T. Wolff [NMEID] to J. Abiss [UNC], dated SeptemDer 25, 1979) and the proposed NMEID verification sampling scheme (letter from T. Buhl [NMEID] to H. Miller [NRC], dated April 23, 1980). 


\section{EXECUTIVE SUMMARY}

On July 16, 1979, a break in the tailings pond dam of the United Nuclear Corporation's Church Rock, New Mexico, mill occurred. Approximately 94 million gallons of the tailings liquid which had been impounded behind this dam were released into an adjacent arroyo along with an estimated 1,100 tons of tailings solids. The spilled solution traveled down the so-called "pipeline arroyo" and into the north branch of the Rio Puerco arroyo. Beyond this point it continued past the location where the north and south branches of the Rio Puerco join immediately northeast of Gallup, New Mexico, continued across the remainder of the state of New Mexico, and extended into the state of Arizona for $\sim 20$ to 25 miles, where the flow of the Rio Puerco terminates.

In September, 1979, Pacific Northwest Laboratory (PNL) responded to a request from the New Mexico Environmental Improvement Division and the U.S. Nuclear Regulatory Commission (NRC) to provide immediate on-site sampling and radionuclide analysis capability. The principal objective of PNL'S work on-site at the United Nuclear Corporation mill was to provide capabilities for immediate analyses of samples which had been collected from the Rio Puerco environment. The conditions for sample preparation and sample analysis were optimized so that the total amount of time required from the time the sample was collected until the data regarding radionuclide concentrations for that sample were available could be less than 48 hours. The concentrations of $210 \mathrm{~Pb},{ }^{226} \mathrm{Ra},{ }^{230} \mathrm{Th}$, and ${ }^{238} \mathrm{U}$ were determined in $\sim 2400$ samples from a survey of the Rio Puerco contamination and from subsequent cleanup operations initiated by the United Nuclear Corporation. The purpose of these later analyses was to determine the effectiveness of the cleanup operations that were ongoing and to evaluate what additional cleanup would be required. This on-site analysis of radioactive contamination constituted the principal task of this project, with the identification of those portions of the arroyo exceeding the NMEID proposed cleanup criteria being the major output.

The following conclusions have been drawn as a result of the investigation of the Church Rock uranium mill tailings pond dam failure: 
total inventory of ${ }^{230}$ Th background in the upper two inches of arroyo sediment is estimated to be $0.30 \mathrm{ci}$.

10. Present inability to differentiate between natural background ${ }^{230}$ Th and contamination-derived ${ }^{230}$ Th prohibits a clear definition of the 230 Th inventory from the tailings pond solution.

11. Sediment samples from the Grand Canyon National Park show no radionuclide levels in excess of normal background. 
1. On-site use of planar intrinsic germanium detectors provides adequate sensitivity and short analysis times for quantitation of $210 \mathrm{~Pb}$, ${ }^{226} \mathrm{Ra},{ }^{230} \mathrm{Th}$, and ${ }^{238} \mathrm{U}$ in sediments/soils.

2. The original sampling plan, which consisted of collecting surface soil/sediment samples at 1000-ft intervals, was adequate to determine those areas which were contaminated and required cleanup.

3. Clustering of samples within arroyo reaches defined by physical (morphological) features would provide a better estimation of the radionuclide spatial variability than did the sampling pattern which was used and which was based on equal distance increments.

4. The revised Rio Puerco cleanup criteria proposed by the NMEID are statistically adequate.

5. Concentrations of $210 \mathrm{~Pb},{ }^{226} \mathrm{Ra}$, and ${ }^{238} \mathrm{U}$ in samples throughout the length of the arroyo are not distinguishable from natural background concentrations.

6. Concentrations of ${ }^{230}$ Th range from background levels to levels elevated considerably greater than background. Plots of ${ }^{230}$ Th concentrations versus distance from the tailings pond show high variability. Therefore, a statistical smoothing function was applied to the data to facilitate interpretation.

7. Sediment samples from two site-variability studies indicate that there is considerable ${ }^{230}$ Th concentration variability within even limited areas of the arroyo (i.e, 5-ft square grids and $25-\mathrm{ft}$ square grids).

8. The concentrations of ${ }^{230}$ Th in the Rio Puerco show an apparent periodicity as a functon of distance. This period is $\sim 2.5 \mathrm{~km}$.

9. The estimated total inventory of ${ }^{230} \mathrm{Th}$ in the Rio Puerco from sampling stake 0-491 is $26.8 \mathrm{Ci}$, based on data from the upper two feet of the core samples. The inventory based on data from the firstterrace and second-terrace samples (upper two inches) is $4.9 \mathrm{Ci}$. The 



\section{CONTENTS}

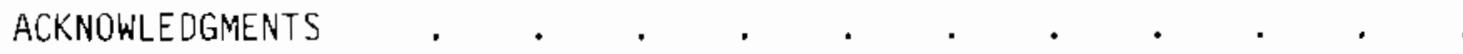

ABSTRACT

EXECUTIVE SUMMARY

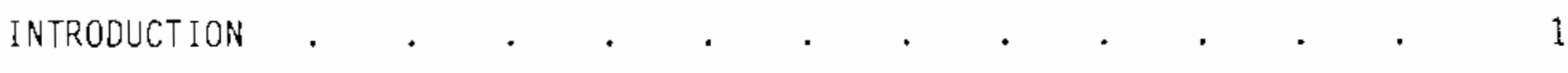

EXPERIMENTAL METHODS . . . . . . . . . . . . . . . 5

SAMPLE COLLECTION

PREPARATION OF SAMPLES FOR INSTRUMENTAL ANRLYSIS . . . . . 6

INSTRUMENTAL ANALYSES OF RADIONUCLIDE CONCENTRATIONS $\quad . \quad . \quad . \quad 8$

Detector Types

Calibration of 9-in. NaI well Crystal for $226 \mathrm{Ra}$

Quantitation . . . . . . . . . . . 8

Calibration of Intrinsic Germanium Detectors . . . . . 10

Selection of Counting Time Interval $\quad . \quad$. . . . 10

Reporting of Uranium Analysis Data . . . . . . . 11

STATISTICAL METHODS . . . . . . . . . . . . . . 12

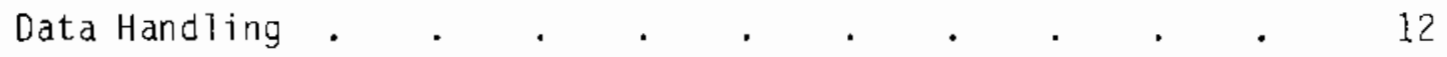

Data Smoothing Functions. . . . . . . . . . 13

EVALUATION OF INSTRUMENTAL COUNTING CAPABILITIES $\quad \cdot \quad \cdot \quad \cdot \quad \cdot \quad \cdot \quad 15$

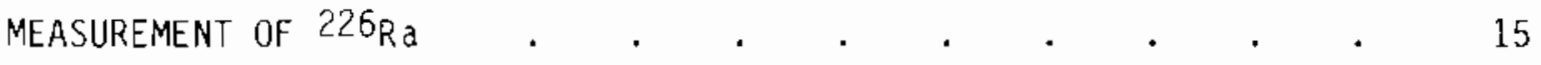

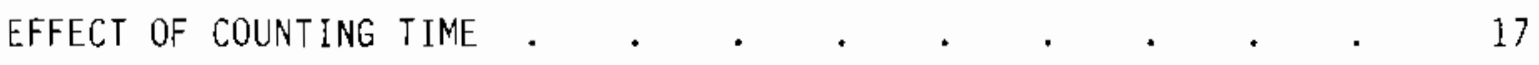

REPLICATE COUNTING OF SAMPLES . $. \quad . \quad . \quad . \quad . \quad . \quad . \quad . \quad 19$

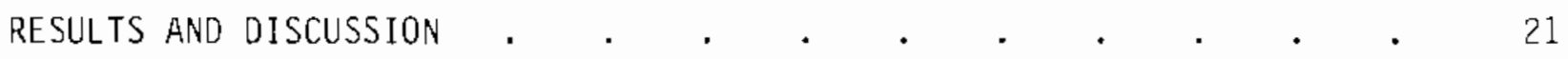

ANALYSIS OF GRAND CANYON SAMPLES $\quad \cdot \quad \cdot \quad \cdot \quad \cdot \quad \cdot \quad \cdot \quad \cdot \quad \cdot \quad 21$

PRESENTATION OF RIO PUERCO SAMPLE DATA $. \quad . \quad . \quad . \quad . \quad . \quad . \quad 24$ 
EVALUATION OF GRID SAMPLING DATA $\quad . \quad$. . . . . . . 27

EVALUATION OF BACKGROUND SAMPLES

ESTIMATION OF 230 Th INVENTORY . $. \quad . \quad . \quad . \quad . \quad . \quad . \quad . \quad . \quad 42$

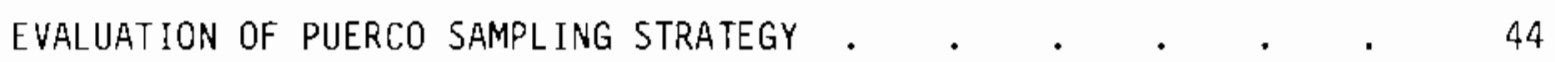

EVALUATION OF VERIFICATION SAMPLING PLAN AND PROPOSED CLEANUP

CRITERIA . . . . . . . . . . . . . . . . . 46

Number of Sample Replicates . . . . . . . . . . 49

EXPERIENCE GAINED FROM THE RIO PUERCO ENVIRONMENTAL STUDY • • • • 53

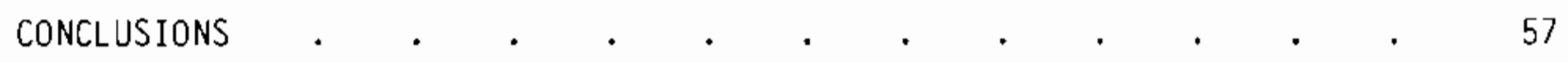

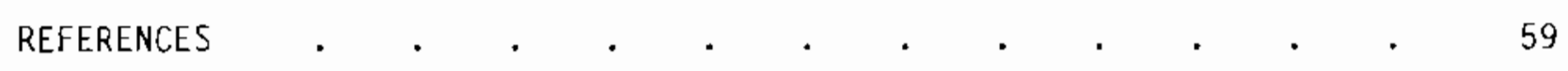

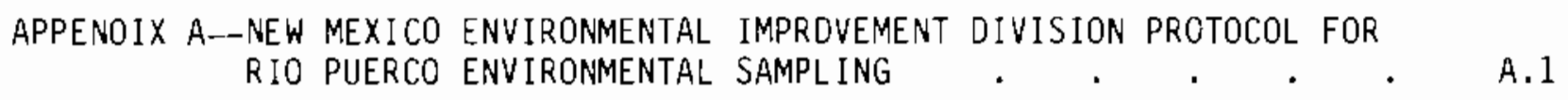

APPENDIX B--DATA FOR RIO PUERCO ENVIRONMENTAL SAMPLES . . . . . . . B.1

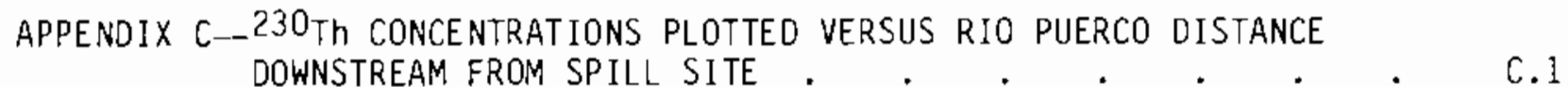

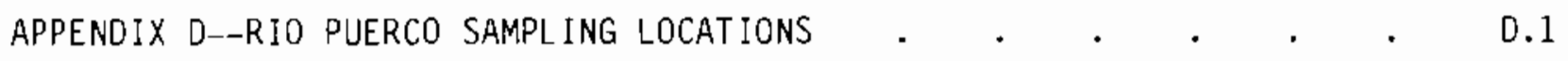

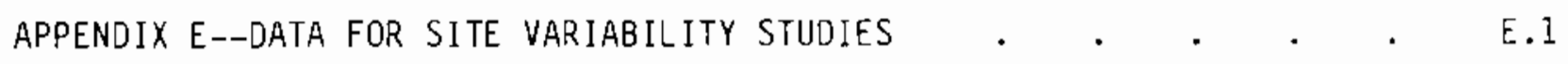

APPENDIX F--PROPOSED REVISED RIO PUERCO CLEANUP CRITERIA — . . . . F.1 
FIGURES

1. Rio Puerco Geographical Location . . . . . . . . . . . 3

2. Grand Canyon Sampling Locations . . . . . . . . . . . 7

3. Typical Spectra from Intrinsic Germanium Diode Count of Soil
Sample ...
.

4. 230 Th Concentration Versus Distance . . . . . . . . . 26

5. Rio Puerco Environmental Variadility Study No. 1 . . . . . . 39

6. Rio Puerco Environmental Variability Study No. 2 . . . . . 40

\section{TABLES}

1. Representative Analysis of UNC Tailings Pond Solution . . . . 2

2. 226Ra Concentrations Measured in Sealed and Unsealed Samples . 16

3. Effect of Counting Time on Precision of Sample Analysis . . 18

4. Variability of Short Counts on Individual Samples--1000-Sec
Counting Time. . . . . . . . . . . .

5. Radionuclide Concentrations in Grand Canyon Samples--pCi/g . . 21

6. Rio Puerco Background Samples . . . . . . . . . . . . . . 22

7. Rio Puerco Samples Having 230 Th Concentrations $>30 \mathrm{pCi} / \mathrm{g}$. $\quad 28$ 



\section{INTRODUCTION}

On July 16, 1979, a oreak in the tailings dam of the United Nuclear Corporation's (UNC) Church Rock, New Mexico, mill occurred. Approximately 94 million gallons of the tailings liquid which had been impounded behind this dam were released into an adjacent arroyo. An estimated 1100 tons of tailings solids also emptied from the dam into the adjoining toe dam and subsequently into the adjacent arroyo. A typical composition of the UNC tailings pond solution is listed in Table 1. The spilled solution traveled down the so-called "pipeline arroyo" and into the north branch of the Rio Puerco arroyo. Beyond this point it continued past the location where the north and south branches of the Rio Puerco join immediately northeast of Gallup, New Mexico, continued across the remainder of the state of New Mexico, and extended into the state of Arizona for 20 to 25 miles. At that point the flow of the Rio Puerco terminates. Figure 1 shows the location of the Rio Puerco in New Mexico.

Subsequent to the release of this tailings pond solution several organizations began environmental sampling and monitoring programs. These organizations include the U.S. Environmentat Protection Agency, the New Mexico Environmenta? Improvement Oivision (NMEID), and personili from the United Nuclear Corporation mine and mill at Church Rock. Intermittent samplings of soil, water, and air were performed ty these organizations between july 16 , 1979, and the middle of September, 1979. At that time Facific Northwest Laboratory (PNL) responded to a request from the U.S. Niclear Regulatory Commission to provide immediate on-site sampling and radionuclide analysis capability. The principal objective of PNL's work on-site at the United Nuclear Corporation mil: was to provide capabilities for immediat: analyses of samples which nas Deen collected from the Rio Puerco environment. Secondarily, assistance and advice on sampling methodology were requested. The conditions for sample preparation and sample analysis were optimized so that the total amount of time required from the time the sample was collected until the data regarding radionuclide concentrations for that sample were available could be less 
TABLE 1. Representative Analysis of UNC Tailings Pond Solution*

\begin{tabular}{|c|c|}
\hline Constituent & $\begin{array}{c}\text { Concentration } \\
(\mathrm{mg} / 1 \text { unless noted otherwise })\end{array}$ \\
\hline Arsenic & 0.07 \\
\hline Barium & $<0.1$ \\
\hline Chloride & 50.0 \\
\hline Chromiurn & 0.15 \\
\hline Cobalt & 0.95 \\
\hline Fluoride & 2.47 \\
\hline Iron & 157.5 \\
\hline Lead & 0.20 \\
\hline Magnesium & 50.8 \\
\hline Manganese & 14.0 \\
\hline Molybdenum & 0.04 \\
\hline Nitrogen (Ammonia) & 47.6 \\
\hline Nitrogen (Nitrate) & 1.9 \\
\hline Nickel & 0.32 \\
\hline $\mathrm{pH}$ & 1.92 \\
\hline Sodium & 519. \\
\hline Sulfate & 4802 \\
\hline Tota? Uranium & 4.09 \\
\hline Total ${ }^{226} \mathrm{Ra}$ & $209.5 \mathrm{pCi} / \mathrm{i}$ \\
\hline Total ${ }^{230}$ Th & $10225.2 \mathrm{pCi} / 1$ \\
\hline
\end{tabular}

than 48 hours. The concentrations of ${ }^{210} \mathrm{~Pb},{ }^{226} \mathrm{Ra},{ }^{230} \mathrm{Th}$, and ${ }^{238} \mathrm{U}$ were determined. Approximately 100 samples per day could be analyzed in the mobile laboratory facility that was placed on-site. 


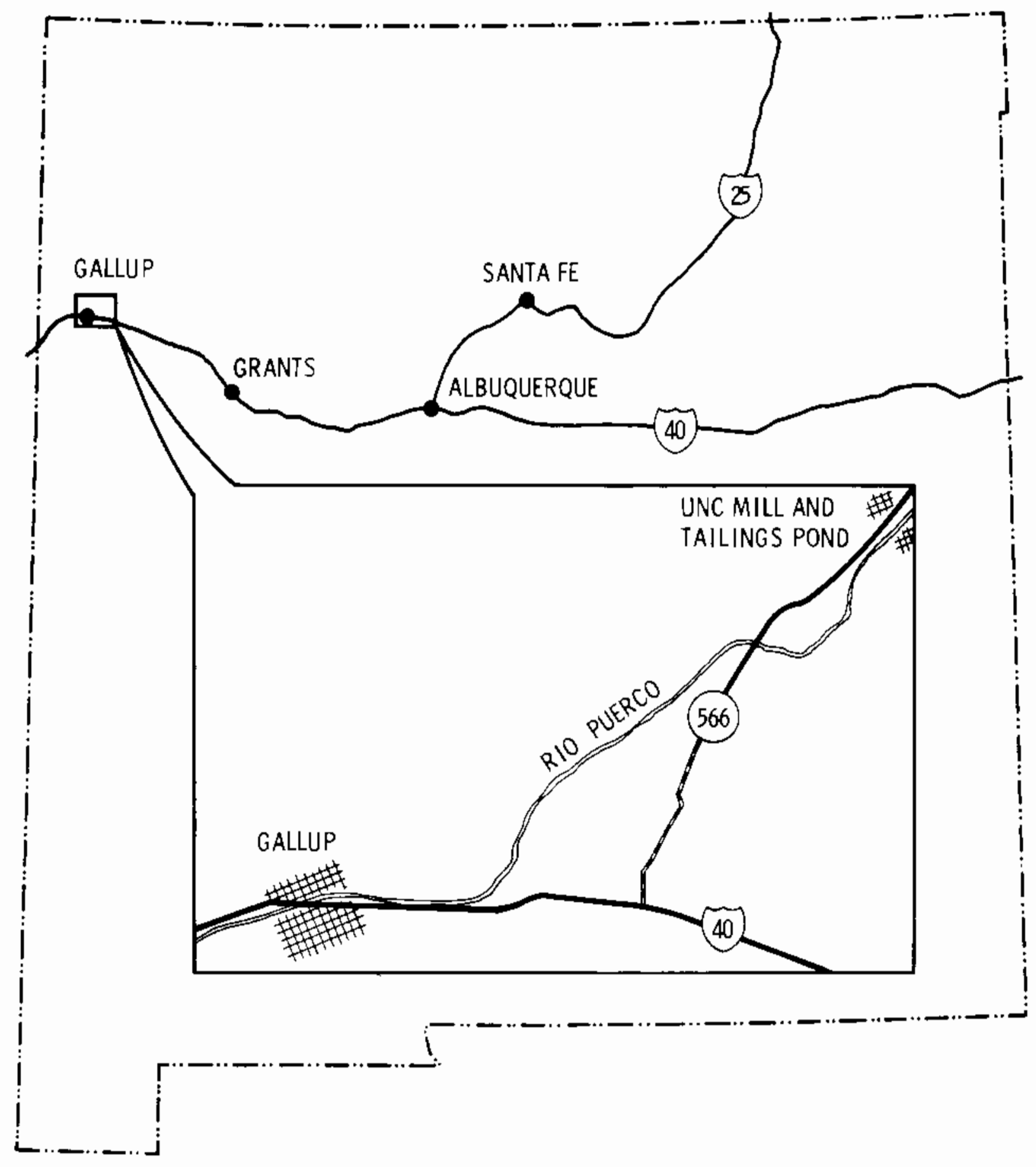

FIGURE 1. Rio Puerco Geographical Location 

EXPERIMENTAL METHODS

\section{SAMPLE COLLECTION}

The United Nuclear Corporation established permanent sampling sites from the point of the tailings dam break downstream to the Arizona-New Mexico border. These sites were established at approximately 500-ft intervals and were marked by the positioning of a stake on both sides of the stream. The first 305 sampling cross-sections were surveyed so that their exact positions could be noted on aerial photographs. From this survey the exact downstream distance of each of these sampling locations was determined. (The remaining sampling locations [through Stake No. 491] were not surveyed and, therefore, their locations cannot be accurately and precisely depicted on aerial photographs.) The New Mexico Environmental Improvement Division sampling protocol required that samples would be taken at every other stake location, or at approximately 1000-ft intervals, along the entire potentially contaminated watercourse. (The exact sampling protocol required by the NMEID is listed in Appendix $A$ of this report.)

A series of sample types was collected at each of the designated sampling locations. These various samples were intended to represent different terraces within the arroyo boundaries (the first terrace is the small plateau immediately adjacent to and higher than the water flow; the second terrace is the next well-defined, higher plateau proceeding away from the water flow), samples from locations of suspected high radionuclide concentrations, samples from areas where pools of suspected contamination had been observed, sediment core samples to evaluate potential penetration of contamination into the arroyo bottom, and samples intended to represent background radionuclide levels along the length of the arroyo.

Surface samples of soil or sediment were collected using a standardized cylindrical scoop of $10.16 \mathrm{~cm}$ (4 in.) diameter and $5 \mathrm{~cm}$ (2 in.) height. At any given sampling location three or four of these standardized scoop samples were composited to provide a total of approximately $1000 \mathrm{~g}$ of sample material. At 
selected locations soil cores were collected using a standard coring device constructed of PVC pipe. The soil cores were divided into 1-ft intervals to a total depth of 3 feet.

Some samples of streambed sediment were also obtained from the Grand Canyon National Park through the efforts of National Park Service employees. These samples were generally collected from backwater areas of the main stream of the Colorado River and from the Little Colorado River and represent samples from the sediment-water interface areas. The exact sampling locations are shown in Figure 2.

PREPARATION OF SAMPLES FOR INSTRUMENTAL ANALYSIS

The soil or sediment sample as received in the laboratory was split into two portions. One portion weighing approximately 750 to $800 \mathrm{~g}$ was double plastic bagged, weighed exactly, and sent to the mobile laboratory van for ${ }^{226} \mathrm{Ra}$ analyses in a 9-in. Nal crystal well counter. A second sample weighing approximately 150 to $200 \mathrm{~g}$ was weighed exactly, oven dried overnight at $110^{\circ} \mathrm{C}$, and then weighed exactly again. This dried sample was then pulverized with a mortar and pestle to a uniform particle size. An aliquot of $60 \mathrm{~g}$ of this ground soil sample was mixed with $9 \mathrm{~g}$ of binder material (either cellulose powder or powdered sugar was used). This sample was then placed into a hardened steel die which, in conjunction with a 25-ton press, was used to press the sample plus binder into a 2-in.-diameter pellet. The pressed pellet was then double plastic wrapped and sent to the mobile laboratory van for instrumental analysis.

Those samples processed prior to October 4, 1979, were split in the above described fashion. At that time it was determined that the analysis of the large volume sample by the 9 -in. well counter for ${ }^{226}$ Ra content was not required. Counting sensitivities were such that all radionuclide concentration data could be obtained from the pressed pellet sample. Therefore, splitting of the sample to provide a 750 - to $800-g$ aliquot was no longer performed. 


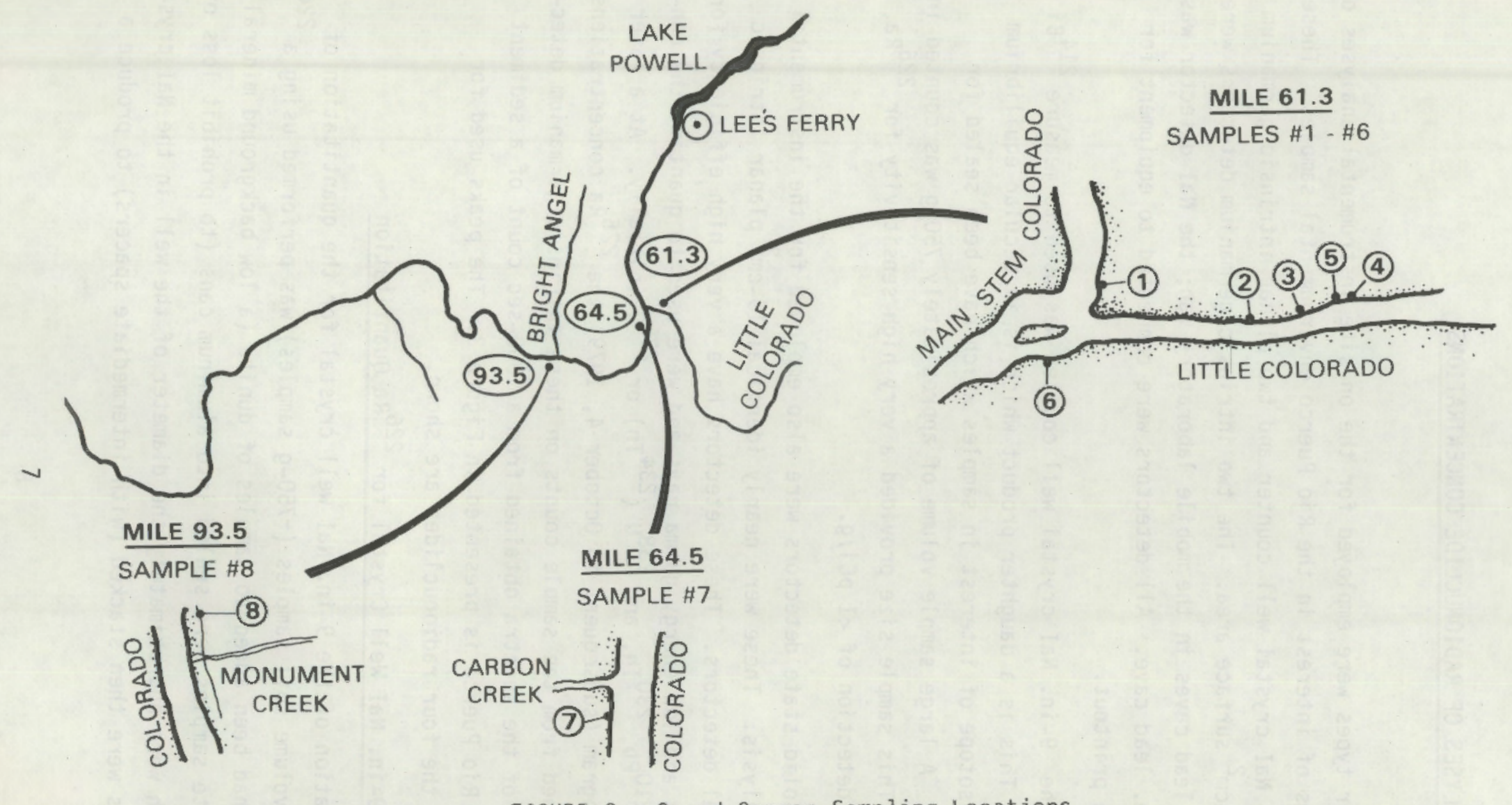

FIGURE 2. Grand Canyon Sampling Locations 


\section{INSTRUMENTAL ANALYSES OF RADIONUCLIDE CONCENTRATIONS}

\section{Detector Types}

Two detector types were employed for the on-site instrumental analyses of the radionuclides of interest in the Rio Puerco environmental samples. These included a 9-in. NaI crystal well counter and two planar intrinsic germanium detectors of $19-\mathrm{cm}^{2}$ surface area. The two intrinsic germanium detectors were housed in 4-in. lead caves in the movile laboratory van; the NaI detector was housed in a 2-in. lead cave. All detectors were connected to equipment for data storage and printout.

Initially the 9-in. NaI crystal well counter was used to measure ${ }^{214} \mathrm{Bi}$ concentrations. This is a daughter product which is in secular equilibrium with the ${ }^{226} \mathrm{Ra}$ isotope of interest in samples which have been sealed for 30 days or more. A large sample volume of approximately $750 \mathrm{~g}$ was counted in this detector. This sample size provided a very high sensitivity for ${ }^{226} \mathrm{Ra}$, with a limit of detection of $<1 \mathrm{pCi} / \mathrm{g}$.

Two other solid state detectors were also employed for the instrumental radionuclide analysis. These were nearly identical $19-\mathrm{cm}^{2}$ planar intrinsic germanium crystal detectors. These detectors have a very high efficiency for detecting $x$-rays and low energy gamma rays and were used to quantify the concentrations of ${ }^{210} \mathrm{~Pb},{ }^{230} \mathrm{Th}$, and ${ }^{238} \mathrm{U}\left({ }^{234} \mathrm{Th}\right)$ or ${ }^{235} \mathrm{U}$ initially. At a later stage in the program (subsequent to 0ctober 4,1979 ) the ${ }^{226} \mathrm{Ra}$ concentrations were also obtained from the sample counts on these intrinsic germanium detectors. A sample of the spectra obtained from a $1000-\mathrm{sec}$ count of a sediment sample from the Rio Puerco is presented in Figure 3. The peaks used for quantitation of the four radionuclides are shown.

Calibration of 9-in. NaI Well Crystal for ${ }^{226}$ Ra Quantitation

The calibration of the 9-in. NaI well crystal for the quantitation of ${ }^{226} \mathrm{Ra}$ in large volume soil samples ( 750-g samples) was performed using a ${ }^{226} \mathrm{Ra}$ standard which had been added to samples of dunite (a low background mineral). The spiked dunite samples were sealed into aluminum cans (to prohibit loss of ${ }^{222} \mathrm{Rn}$ gas) which were approximately the diameter of the well in the NaI crystal. Three cans were then stacked (with intermediate spacers) to produce a 


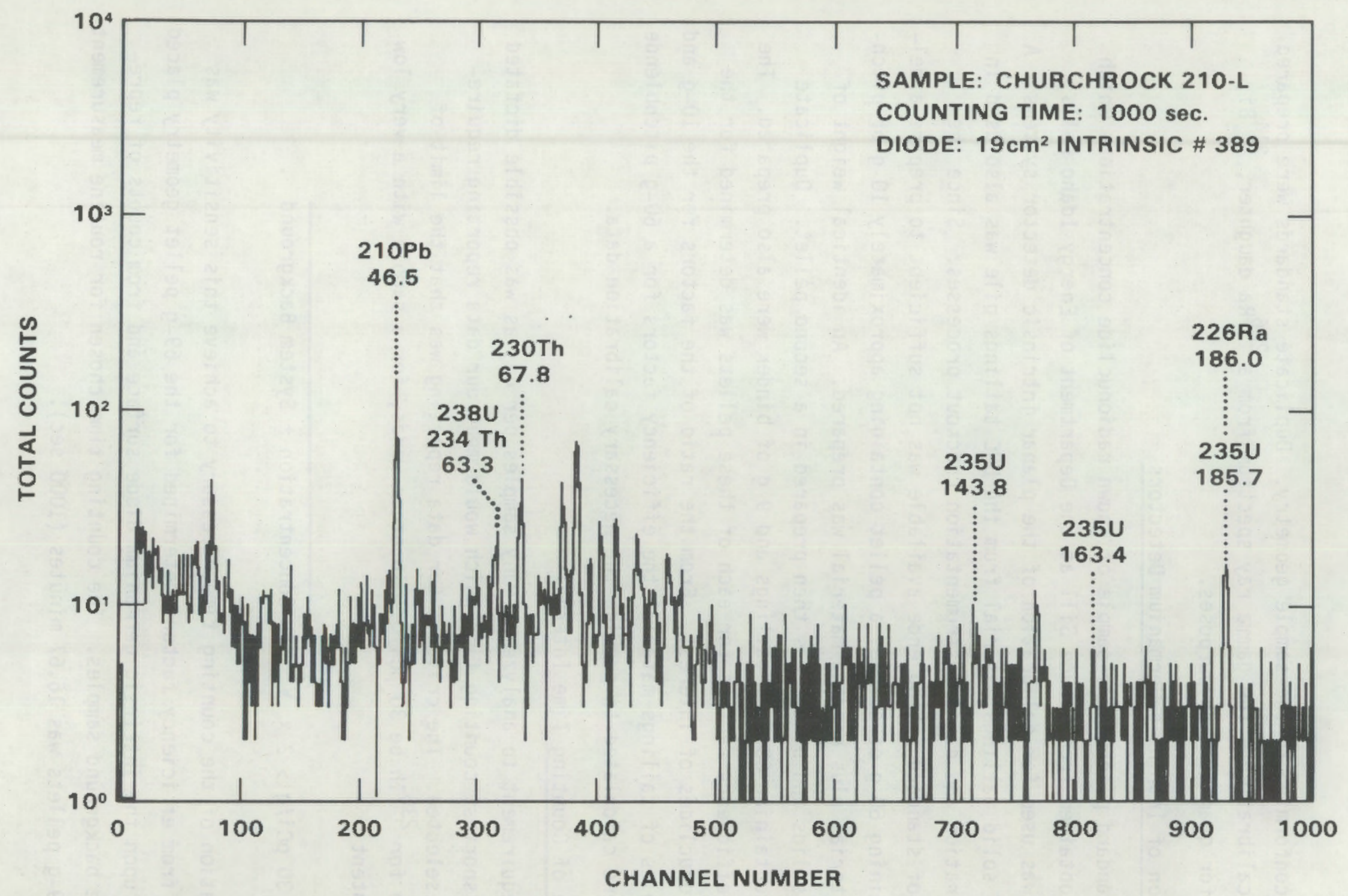

FIGURE 3. Typical Spectra from Intrinsic Germanium Diode Count of Soil Sample 
standard conforming to the sample geometry. Duplicate standards were prepared for this calibration. The gamma ray spectrum from a ${ }^{226} \mathrm{Ra}$ daughter, ${ }^{214} \mathrm{Bi}$, was used for quantitation purposes.

Calibration of Intrins ic Germanium Detectors

A standard pitchblende sample of known radionuclide concentrations which had been obtained from Claude Sill at the Department of Energy Idaho Falls facility was used for calibration of the planar intrinsic detector systems. A sample of solid tailings material from the UNC tailings pile was also used in the calibration and daily instrumentation checkout processes. Since the quantity of standard pitchblende available was not sufficient to prepare a pellet containing $60 \mathrm{~g}$ of sample, a pellet containing approximately $10 \mathrm{~g}$ of pitchblende material plus binding material was prepared. An identical weight of tailings solids and binder was then prepared in a second pellet. Ouplicate pellets containing $60 \mathrm{~g}$ of tailings and $\mathrm{g} \mathrm{g}$ of binder were also prepared. The counting efficiency factors for each of these pellets was determined for the four radionuclides of interest. From the ratio of the factors for the $10-g$ and 60-g samples of tailings material the efficiency factors for a 60-g pitchblende sample were calculated to provide the necessary calibration data.

Selection of Counting Time Interval

A requirement to analyze as many samples per day as was possible dictated that the shortest counting time which would meet our data reporting requirements be selected. The criteri, for data reporting was that the limit of detection for ${ }^{230}$ Th be $30 \mathrm{pCi} / \mathrm{g}$. In other words, for a sample with a very low ${ }^{230}$ Th content

$30 \mathrm{pCi} / \mathrm{g}>2 \times \sqrt{230}$ Th Concentration + System Background.

An estimation of the counting time necessary to achieve this sensitivity was obtained from efficiency factors determined for the 69-g pellet geometry placed directly upon the intrinsic germanium diode surface and from counts of representative background samples. The counting time chosen for routine measurement of the $69-g$ pellets was 16.67 minutes $(1000 \mathrm{sec})$. 
Reporting of Uranium Analysis Data

Uranium data which were obtained from direct counting of the samples on the intrinsic germanium detectors were reported in two different manners. Initially (from September 23, 1979, to October 16, 1979) the uranium concentrations were reported as ${ }^{235} \mathrm{U}$ and were based upon quantitation of the 163.4-keV peak. Since only approximately $5 \%$ of the decays go through this energy and ${ }^{235} U$ has only a 0.72 isotopic abundance, the limit of detection when using this peak is rather high, and the counting error associated with the concentration estimate is large. Sufficient data were available by mid-October to allow the measurement of ${ }^{234} \mathrm{Th}$ for ${ }^{238} \mathrm{U}$ quantitation. Therefore, the method of quantifying the uranium concentration was changed to one using two gamma rays (at 63 and $93 \mathrm{keV}$ ) from ${ }^{234} \mathrm{Th}$ and reporting the concentrations as ${ }^{238} \mathrm{U}$. This method assumes secular equilibrium between the parent ${ }^{238_{U}}$ and the daughter ${ }^{234} \mathrm{Th}$. Also implicit in this approach is the assumption that al1 ${ }^{234}$ Th in the sample came from ${ }^{238} U$ in the sample. This situation might not be met if a solution rich in thorium had recently been separated from the uranium, released into the environment as during the tailings pond dam failure, and analyzed relatively soon after the initial Th/U separation. Since the half-life of ${ }^{234}$ Th is approximately 24 days, more than three half-lives had passed between the time of the tailings solution release and the time that we began to use the ${ }^{234}$ Th measurement for the ${ }^{238} \mathrm{U}$ quantitation. Also, the $94 \times 10^{6}$ gallons of tailings solution that were released by the dam failure represents a uranium inil1 waste composite of at least 104 days of operation. Hence, there was considerable time for any excess ${ }^{234}$ Th present in the solution to decay prior to the time that the Rio Puerco soil/sediment samples were analyzed. Additiona)ly, any excess ${ }^{234}$ Th in the solution that contaminated the sediment/soil samples would serve to overestimate the actual ${ }^{238} \mathrm{U}$ concentration of the sample. That is, any errors would be in a conservative direction and would serve as an additional safety precaution. 
STATISTICAL METHODS

Data Handing

The descriptive statistical effort for the data consisted of three steps and made extensive use of PNL's computer facilities. The first step involved data editing for the purpose of correcting errors and arranging the data in an order convenient for plotting. The second step consisted of computing summary statistics such as group means and confidence intervals and of filtering the data. The third step was to plot the data for graphical presentation.

Most of the data were received from the state of New Mexico Environmental Improvement Division on 80-column punched cards. The first set of cards was followed by about 100 additions and numerous corrections given to us as listings of the new aata. To all of these data we added the distances from stake zero which were determined from maps. The data were first stored as a file on the high speed disk at the central computing facility. Backup copies were stored on mini-floppy disks kept in offices. The data editing step was implemented using a specially written BASIC language program. This program permitted an interactive examination and editing of the data for any sample. The program was used to correct later errors and to add new sample data to the computer file.

After the data were edited, several small BASIC and FORTRAN Ianguage programs were written to calculate confidence intervals and local averages and to filter the data. These programs utilized the stake and sample type coding developed by the NMEID to simplify the selection of samples for each computation. The output of these programs was also stored on computer disk.

The final step was to present the data, displayed in a manner convenient for human use. One special purpose and one general purpose computer program were used for this step. The special purpose program simply reformatted the data, mostly by inserting spaces between the columns of data and by replacing the missing value codes with blanks, then printed a listing of the data. The general purpose program was used to produce plots of the data. The program used was BMDP6D, the scatter plotting program from the UCLA Biomedical Computer Programs P-series. For this work the program was used to plot 
radionuclide concentrations against distance along the arroyo. Plotting on a computer line printer was used since this is much faster and much less expensive than using an $x-y$ plotter. The program finds the subset of data to use for each plot, computes summaries and descriptions of each plot, and automatically annotates the axis of the plots. BMDP6D was used to produce all the plots of data used during the preliminary data analysis steps. The final data presentations were produced using an $x-y$ plotter.

\section{Data Smoothing Functions}

Two types of data smoothing functions were used. The first of these was a weighted average type of smoothing. This weighting was performed between samples collected at individual sampling locations. (Unweighted averages were used when combining data from different sampling locations.) This type of smoothing considers the more precise sample measurements (those with smaller counting errors) to be more important than the less precise sample values (those with higher counting errors). Data were first segregated by sample type or sampling site so that all like samples were analyzed independently of all other types. Replicates at each sampling site were then averaged. The standard statistical weighting factors are the inverse of the variances (counting errors squared). Let $x_{1}, \ldots, x_{n}$ represent the replicates for one type of sample at one sampling point and $S_{1}, \ldots, s_{n}$ represent the corresponding counting errors. Then the weighted mean is:

$$
M=\frac{\sum_{i=1}^{N} x_{i} / s_{i}^{2}}{\sum_{i=1}^{N} 1 / s_{i}^{2}}
$$

and the corresponding standard error of the mean is:

$$
S . E .=\frac{1}{\sum_{i=1}^{N} 1 / S_{i}^{2}}
$$


Under the assumption that at each sampling point the repiicates are sampled from the same statistical population, it is inappropriate to include a betweensamples term in this standard error.

A second type of smoothing was performed, again for each type of sample, over a few successive sampling points. One of the simpliest low pass filters of time series analyses was used called a "First Order Low Pass Filter" (Ontes and Enocnson 1978, Chatfield 1975). It is computed by:

$$
Y_{j}=(a) Z_{j}+(1-a) Y_{j-1}
$$

where $Z_{j}$ is the series of data values, $Y_{j}$ is the series of filtered data values, and "a" is the filter factor. A filter factor of 0.25 was used to fiiter both the weighted means and standard errors of the means. This value for the filtering factor was determined by repeatedly evaluating the data for the first $10,000 \mathrm{~m}$ of the arroyo with various filter factors then arbitrarily choosing what subjectively was the "best" filter factor value. The "a" value was chosen to just average out noise but not remove any significant patterns from the data. This filtering scheme uses data from a few previous data points with an exponential weighting scheme. Data points more than 5 to 8 prior to the current point are essentially swamped out or weighted as almost zero so that they no longer influence the computations. This smoothing also gave estimates of the standard error of the smoothed averages, and these were used for the approximate confidence intervals plotted. 


\section{EVALUATION OF INSTRUMENTAL COUNTING CAPABILITIES}

MEASUREMENT OF $226_{\mathrm{Ra}}$

Measurements of ${ }^{226}$ Ra were made on-site using either of two systems, a 9-in. NaI well crystal and $19-\mathrm{cm}^{2}$ planar intrinsic germanium detectors. The $186 \mathrm{keV}$ y ray from ${ }^{226}$ Ra decay was used for quantitation by the intrinsic detectors. For the NaI measurements ${ }^{226} \mathrm{Ra}$ itself was not measured but rather the $Y$ rays from a daughter product, ${ }^{214} \mathrm{Bi}$, were quantified. In order to caiculate the ${ }^{226}$ Ra concentrations from measurements of this type it must be assumed 1) that the ${ }^{214} \mathrm{Bi}$ is in secular equilibrium with ${ }^{226} \mathrm{Ra}$ and 2) that one of the ${ }^{226} \mathrm{Ra}$ daughters, ${ }^{222} \mathrm{Rn}$, a gas, is not lost from the sample during the decay to $214 \mathrm{Bi}$.

To evaluate the validity of the assumption that no radon gas is lost from the sample after it has been pressed into a pellet, several samples were sealed into individual aluminum cans, which are known to retain radon gas. The samples in these cans were then counted on a multi-dimensional $\mathrm{NaI}$ detector system [see Wogman et a1. (1967) for a complete description of this counting system] at several different time interyals to determine if the apparent ${ }^{226} \mathrm{Ra}$ concentration varied as a function of time. In addition, other subsamples from these individual samples were also counted at the Church Rock site in the 9-in. $\mathrm{NaI}$ well counter. The data from this series of counts are presented in Table 2. The apparent ${ }^{226}$ Ra concentration increased in each of the seven samples that were examined. This increase ranged from $5 \%$ to $37 \%$ of the original ${ }^{226} \mathrm{Ra}$ concentration measured. These data indicate that during the oven drying, sample pulverizing, and pellet pressing operations that prepare a pe]letized sample for instrumental counting there is some loss of ${ }^{222}$ Rn gas from the sample into the laboratory environment. Based on data from these seven samples it would appear that while the loss is definitely measureable it is not of such magnitude as to affect the overail interpretation of the data gathered from direct counts on nonsealed pellets.

Table 2 also shows a comparison of the radium concentrations determined for the sealed samples versus those determined for unsealed samples in the g-in. well counter. There is, in general, good agreement between the data 
TA8LE 2. ${ }^{226} \mathrm{Ra}$ Concentrations Measured in Sealed and Unsealed Samples

\begin{tabular}{|c|c|c|c|c|c|c|}
\hline & & & & Unsealed & Samp les & \\
\hline Muiti-dimen & aled Samp & $\frac{\text { s* }^{*}}{\operatorname{ting}}$ System & 9-in. & Well Counter & Ir & $\begin{array}{l}\text { trinsic } \\
\text { ium Detector }\end{array}$ \\
\hline Sample & $\begin{array}{l}\text { Date } \\
\text { Counted }\end{array}$ & $\begin{array}{c}{ }^{226} \mathrm{Ra} \\
\text { Concentration } \\
\mathrm{pCi} / \mathrm{g} \\
\end{array}$ & $\begin{array}{l}\text { Oate } \\
\text { Counted }\end{array}$ & $\begin{array}{c}226_{\mathrm{Ra}} \\
\text { Concentration } \\
\mathrm{pC} \mathrm{i} / \mathrm{g}\end{array}$ & $\begin{array}{l}\text { Date } \\
\text { Counted }\end{array}$ & $\begin{array}{c}{ }^{226} \mathrm{Ra} \\
\text { Concentration } \\
\mathrm{pC} i / \mathrm{g} \\
\end{array}$ \\
\hline $203-L$ & $\begin{array}{l}10 / 03 / 79 \\
10 / 04 / 79 \\
10 / 05 / 79\end{array}$ & $\begin{array}{l}0.87 \pm 0.012 \\
0.89 \pm 0.011 \\
0.91 \pm 0.012\end{array}$ & $09 / 28 / 79$ & $0.75 \pm 0.12$ & & \\
\hline $210-L$ & $\begin{array}{l}10 / 03 / 79 \\
10 / 04 / 75 \\
10 / 05 / 79 \\
08 / 11 / 80\end{array}$ & $\begin{array}{l}8.24 \pm 0.039 \\
8.65 \pm 0.038 \\
8.94 \pm 0.036 \\
10.5 \pm 0.21\end{array}$ & $09 / 28 / 79$ & $6.8 \pm 0.70$ & $09 / 10 / 80$ & $6 \pm 4$ \\
\hline $217-\mathrm{L}$ & $\begin{array}{l}10 / 03 / 79 \\
10 / 04 / 79 \\
10 / 05 / 79 \\
08 / 24 / 80\end{array}$ & $\begin{array}{l}3.13 \pm 0.027 \\
3.27 \pm 0.026 \\
3.40 \pm 0.025 \\
3.88 \pm 0.078\end{array}$ & $09 / 28 / 79$ & $2.2 \pm 0.23$ & $09 / 10 / 80$ & $5.6 \pm 2.3$ \\
\hline $224-\mathrm{L}$ & $\begin{array}{l}10 / 02 / 79 \\
10 / 24 / 79 \\
08 / 25 / 80\end{array}$ & $\begin{array}{l}1.14 \pm 0.013 \\
1.34 \pm 0.058 \\
1.43 \pm 0.029\end{array}$ & $09 / 28 / 79$ & $0.76 \pm 0.11$ & $09 / 10 / 80$ & $2.3 \pm 2.2$ \\
\hline EID 8-B & $\begin{array}{l}10 / 02 / 79 \\
10 / 24 / 79 \\
08 / 22 / 80\end{array}$ & $\begin{array}{l}2.08 \pm 0.024 \\
2.69 \pm 0.029 \\
2.84 \pm 0.057\end{array}$ & $09 / 27 / 79$ & $2.5 \pm 0.34$ & $09 / 10 / 80$ & $3.7 \pm 2.5$ \\
\hline 102 Mud Cracks & $\begin{array}{l}10 / 02 / 79 \\
10 / 24 / 79 \\
08 / 21 / 80\end{array}$ & $\begin{array}{l}4.07 \pm 0.033 \\
4.74 \pm 0.098 \\
4.95 \pm 0.099\end{array}$ & $09 / 28 / 79$ & $5.5 \pm 0.63$ & & \\
\hline J0 $3-B$ & $\begin{array}{l}10 / 03 / 79 \\
10 / 04 / 79 \\
10 / 05 / 79\end{array}$ & $\begin{array}{l}1.87 \pm 0.027 \\
1.89 \pm 0.026 \\
1.98 \pm 0.026\end{array}$ & $09 / 28 / 79$ & $1.6 \pm 0.19$ & & \\
\hline
\end{tabular}

*Samples were sealed in cans on October 1, 1979. 
obtained from the two types of counting systems and two types of sample containment systems. Additional data presented in Table 2 show the comparisons between the ${ }^{226} \mathrm{Ra}$ concentrations determined by intrinsic germanium detector systems and by the multi-dimensional counting systems, the most accurate method of determination. These data indicate that the intrinsic germanium detectors do give reliable estimates of ${ }^{226} \mathrm{Ra}$ concentrations in pressed soil pellets. For three of the four cross-checks of the determinations by the intrinsic detectors, the original intrinsic counts yielded conservative, slight overestimates of ${ }^{226} \mathrm{Ra}$ concentrations. These relatively minor deviations are offset by the sensitivity and convenience of using the intrinsic detectors as tools for rapid radiologic assessments of environmental samples.

\section{EFFECT OF COUNTING TIME}

The 1000-sec counting time that was used routinely for on-site sample andysis was selected based upon the criteria which would provide a limit of detection for ${ }^{230}$ Th concentrations of approximately $30 \mathrm{pCi} / \mathrm{g}$. To compare the counting statistics precision for these short counts with what might be obtained for the same counting systems using a longer counting time, several samples were counted for both $1000-\mathrm{sec}$ and longer counting intervals. The data from these counts are given in Table 3 . These data are for two sets of samples; one of these sets of eight samples was counted for intervals of 1000 and $2000 \mathrm{sec}$. Some improvement in counting statistics was obtained by this doubling of the counting time. For some of the ${ }^{230}$ Th concentrations which were measured, significantly different concentrations were calculated for the 1000 - and 2000-sec counts; however, in all cases the two sigma confidence intervals did overlap. Also presented in this table are some data comparing $1000-\mathrm{sec}$ counts with $-500-m i n$ counts on 5 ix individual samples. Similar data are obtained. The counting statistics on the longer counts are considerably better but the concentrations of the radionuclides generally are not significantly different at their two sigma values. (However, one set of ${ }^{230} \mathrm{Th}$ and four sets of ${ }^{238} \mathrm{U}$ data were significantly different.) The primary net effect of counting samples for a longer period of time is to provide a narrower conf $i$ dence interval for the data which are reported. Since one of the overriding 
TABLE 3. Effect of Counting Time on Precision of Sample Analys is

\begin{tabular}{|c|c|c|c|c|c|c|c|}
\hline \multirow[b]{2}{*}{ SAMPLE } & \multirow[b]{2}{*}{ Counting } & \multirow[b]{2}{*}{ Time } & \multicolumn{5}{|c|}{ sotope Concentration--pCi/g } \\
\hline & & & ${ }^{230} \mathrm{Th} \pm \sigma^{1}$ & $226_{R}$ & $R a \pm 0$ & $210 \mathrm{pb} \pm \sigma$ & $238 \mathrm{U} \pm \mathrm{a}$ \\
\hline \multirow[t]{2}{*}{$\overline{0-4-0-5-1}$} & 1,000 & $\mathrm{Sec}$ & $16 \pm 9.8$ & & $N D^{2}$ & $4.3 \pm 1.2$ & $-1.1 \pm 0.99$ \\
\hline & 28,100 & $\mathrm{Sec}$ & $20 \pm 2.5$ & 0.50 & \pm 0.40 & $2.8 \pm 0.30$ & $4.7 \pm 0.30$ \\
\hline \multirow[t]{2}{*}{$0-3-0-5$} & 1,000 & $\mathrm{Sec}$ & $3.7 \pm 8.7$ & & ND & $2.8 \pm 1.1$ & $1.4 \pm 0.92$ \\
\hline & 27,600 & $\mathrm{Sec}$ & $12 \pm 0.30$ & 0.60 & \pm 0.30 & $2.3 \pm 0.20$ & $3.2 \pm 0.20$ \\
\hline \multirow[t]{2}{*}{$0-2$} & 1,000 & $\mathrm{Sec}$ & $17 \pm 8.6$ & & ND & $1.1 \pm 0.93$ & $0.0 \pm 0.88$ \\
\hline & 27,100 & $\mathrm{Sec}$ & $9.0 \pm 1.6$ & 0.30 & \pm 0.30 & $.5 \pm 0.20$ & $3.9 \pm 0.20$ \\
\hline \multirow[t]{2}{*}{$0-4$} & 0 & $\mathrm{Sec}$ & $-10 \pm 9.1$ & & ND & $1.0 \pm 0.95$ & $0.90 \pm 1.0$ \\
\hline & 27,100 & Sec & $18 * 1.7$ & 0.70 & \pm 0.30 & $2.5 \pm 0.20$ & $3.2 \pm 0.20$ \\
\hline \multirow[t]{2}{*}{$0-3-D-3$} & 1,000 & $\operatorname{Sec}$ & $19 \pm 11$ & & ND & $1.3 \pm 1.7$ & $-0.20 \pm 1.1$ \\
\hline & 30,700 & $\mathrm{Sec}$ & $10 \pm 1.0$ & & \pm 0.20 & $1.1 \pm 0.10$ & $2.6 \pm 0.10$ \\
\hline \multirow[t]{2}{*}{$0-4-0-5-4$} & 1,000 & $\mathrm{Sec}$ & $23 \pm 13$ & & NO & $4.4 \pm 1.7$ & $-1.6 \pm 0.91$ \\
\hline & 30,700 & $\mathrm{Sec}$ & $3 \pm 1.4$ & 0.30 & \pm 0.20 & $1.5 \pm 0.20$ & $3.1 \pm 0.10$ \\
\hline \multirow[t]{2}{*}{ EID-1001 } & 1,000 & Sec & $250 \pm 56$ & 150 & \pm 16 & $190 \pm 7.2$ & $330 \pm 7.8$ \\
\hline & 2,000 & $\mathrm{Sec}$ & $200 \pm 52$ & 130 & \pm 11 & $200 \pm 6.8$ & $340 \pm 6.8$ \\
\hline \multirow[t]{2}{*}{ EID-1002 } & 000 & Sec & $0 \pm 6$ & 20 & \pm 1 & $140 \pm 8.6$ & $280 \pm 8.6$ \\
\hline & 2,000 & Sec & $210 \pm 36$ & 100 & \pm 9.9 & $140 \pm 4.5$ & $240 \pm 4.5$ \\
\hline \multirow[t]{2}{*}{ E[0-1003 } & 1,000 & $\mathrm{Sec}$ & $130 \pm 43$ & 79 & \pm 12 & $88 \pm 5.2$ & $170 \pm 5.8$ \\
\hline & 2,000 & $\mathrm{Sec}$ & $110 \pm 29$ & 9 & \pm 8.1 & $93 \pm 3.7$ & $160 \pm 3.7$ \\
\hline \multirow[t]{2}{*}{ EID-1004 } & 1,000 & Sec & $-30 \pm 39$ & 37 & \pm 8.3 & $57 \pm 5.2$ & $95 \pm 5.1$ \\
\hline & 2,000 & $\mathrm{Sec}$ & $61 \pm 27$ & 41 & \pm 5.8 & $54 \pm 3.8$ & $94 \pm 3.6$ \\
\hline \multirow[t]{2}{*}{ EID-1005 } & 1,000 & $\mathrm{Sec}$ & $0.0 \pm 11$ & -0.50 & \pm 1.6 & $1.9 \pm 0.90$ & $3.0 \pm 1.1$ \\
\hline & 2,000 & $\mathrm{Sec}$ & $-4.3 \pm 5.9$ & 0.45 & \pm 1.2 & $1.7 \pm 0.76$ & $3.2 \pm 0.69$ \\
\hline \multirow[t]{2}{*}{ EIO-1006 } & 1,000 & $\mathrm{Sec}$ & $12 \pm 11$ & 1.0 & \pm 1.9 & $2.9 \pm 1.5$ & $4.5 \pm 1.3$ \\
\hline & 2,000 & $\mathrm{Sec}$ & $-9.3 \pm 6.2$ & 0.18 & \pm 1.1 & $1.5 \pm 0.63$ & $1.5 \pm 0.65$ \\
\hline \multirow[t]{2}{*}{ E ID-1007 } & 1,000 & $\mathrm{Sec}$ & $2.5 \pm 11$ & 4.1 & \pm 2.0 & $1.7 \pm 1.1$ & $2.4 \pm 1.0$ \\
\hline & 2,000 & $\mathrm{Sec}$ & $-8.0 \pm 7.8$ & 0.35 & \pm 0.40 & $1.6 \pm 1.0$ & $2.9 \pm 0.90$ \\
\hline \multirow[t]{2}{*}{ EID-1008 } & 1,000 & $\mathrm{Sec}$ & $-5.3 \pm 12$ & 4.6 & \pm 1.8 & $5.9 \pm 1.5$ & $1.3 \pm 1.4$ \\
\hline & 2,000 & $\mathrm{Sec}$ & $-7.1 \pm 7.7$ & 1.2 & \pm 1.3 & $4.2 \pm 0.95$ & $3.0 \pm 0.91$ \\
\hline
\end{tabular}

1-o--counting error. 2-ND--not determined. 
criteria in our on-site analysis program was to process and analyze as great a number of samples as was possible while still meeting the $30 \mathrm{pCi} / \mathrm{g}{ }^{230} \mathrm{Th}$ detection limit, we chose to use the minimum counting time possible. This was the 1000-sec counting time.

REPLICATE COUNTING OF SAMPLES

The data which were obtained from the short versus long counting time studies indicated that concentrations reported could be highly variable for some isotopes in some samples. To obtain a better indication of the variability that could be expected, each of two samples was sequentially counted seven times. The data from this study are presented in Table 4. The greatest variability was shown for the calculated concentrations of ${ }^{230}$ Th. These data indicate (Sample APR-106-P-11) that it may be possible to underestimate the ${ }^{230}$ Th concentrations such that they appear not to exceed the cleanup criteria when in fact they do. For this reason samples that may be used to identify regions requiring a significant amount of cleanup activity should be counted for a longer period of time in order to obtain better counting statistics. 
TABLE 4. Variability of Short Counts on Individual Samples-$1000-\mathrm{Sec}$ Counting Time

\begin{tabular}{|c|c|c|c|c|}
\hline \multirow[b]{2}{*}{ SAMPLE } & \multicolumn{4}{|c|}{ Isotope Concentration-- $\mathrm{pC} \mathrm{i} / \mathrm{g}$} \\
\hline & ${ }^{230} \mathrm{Th} \pm \sigma$ & $226_{\mathrm{Ra}} \pm \sigma$ & $210 \mathrm{~Pb} \pm \sigma$ & $238 \mathrm{U} \pm 0$ \\
\hline \multirow[t]{6}{*}{ APR-106-P-11 } & $37 \pm 13$ & $-0.70 \pm 2.5$ & $6.5 \pm 1.4$ & $11 \pm 1.4$ \\
\hline & $46 \pm 13$ & $-0.50 \pm 2.5$ & $7.3 \pm 1.4$ & $7.2 \pm 1.4$ \\
\hline & $81 \pm 14$ & $-2.2 \pm 2.4$ & $9.0 \pm 1.5$ & $10 \pm 1.5$ \\
\hline & $31 \pm 13$ & $1.2 \pm 2.5$ & $5.4 \pm 1.6$ & $5.3 \pm 1.4$ \\
\hline & $46 \pm 13$ & $-0.40 \pm 2.4$ & $7.7 \pm 1.4$ & $8.1 \pm 1.4$ \\
\hline & $27 \pm 13$ & $2.8 \pm 2.7$ & $5.8 \pm 1.4$ & $6.5 \pm 1.4$ \\
\hline $\bar{x} \pm \hat{\sigma}_{x}^{2}$ & $\frac{41 \pm 13}{44 \pm 18}$ & $\frac{1.8 \pm 2.3}{0.29 \pm 1.7}$ & $\frac{9.0 \pm 1.4}{7.2 \pm 1.4}$ & $\frac{6.3 \pm 1.3}{7.7 \pm 2.0}$ \\
\hline \multirow[t]{6}{*}{ APR-106-T-11 } & $21 \pm 12$ & $1.2 \pm 1.8$ & $-0.60 \pm 1.4$ & $4.2 \pm 1.2$ \\
\hline & $5.3 \pm 13$ & $1.3 \pm 2.0$ & $2.7 \pm 1.5$ & $4.5 \pm 1.4$ \\
\hline & $11 \pm 11$ & $3.1 \pm 1.9$ & $1.2 \pm 1.4$ & $3.9 \pm 1.2$ \\
\hline & $5.3 \pm 12$ & $2.4 \pm 1.6$ & $3.3 \pm 2.3$ & $2.4 \pm 1.2$ \\
\hline & $-0.70 \pm 12$ & $0.80 \pm 1.9$ & $1.5 \pm 1.4$ & $3.4 \pm 1.4$ \\
\hline & $11 \pm 10$ & $1.3 \pm 1.6$ & $1.5 \pm 1.3$ & $3.2 \pm 1.3$ \\
\hline $\bar{x} \pm \hat{\sigma}_{x}$ & $-\frac{11 \pm 10}{9.1 \pm 6.8}$ & $\frac{2.6 \pm 1.9}{1.8 \pm .87}$ & $\frac{1.9 \pm 1.2}{1.6 \pm 1.2}$ & $\frac{3.4 \pm 1.1}{3.6 \pm 0.70}$ \\
\hline
\end{tabular}

1- o--Counting error.

2- $\hat{\sigma}_{x}$-between-5amples error. 


\section{RESULTS AND DISCUSSION}

\section{ANALYSIS OF GRAND CANYON SAMPLES}

Eight samples were received from personnel of the Grand Canyon National Park who were concerned regarding the possible contamination of the Little Colorado and the Colorado rivers by the Church Rock Mill tailings pond solution since the Rio Puerco is a part of the Colorado River drainage system. Six of the samples contained sufficient sedimentary material for instrumental analysis; the remaining two samples (GC-2 and GC-4) were predominately water and were not andyzed. Counting times were approximately $1000 \mathrm{~min} / \mathrm{sample.} \mathrm{The}$ data for the radionuclide concentrations in these samples are listed below in Table 5. These concentrations reflect typical natural background levels even though these particular samples were collected in areas expected to indicate the presence of any potential contamination from the Church Rock dam failure. For comparison purposes, data for all of the background samples collected along the Rio Puerco are listed in Table 6.

TABLE 5. Radionuclide Concentrations in Grand Canyon Samples-aci/g

\begin{tabular}{|c|c|c|c|c|}
\hline Sample Number 1 & $230 T h \pm \sigma^{2}$ & $22.6 \mathrm{Ra} \pm \sigma$ & $210 \mathrm{~Pb} \pm 0$ & $238 U \pm 0$ \\
\hline Grand Canyon No. 1 & $0.14 \pm 1.1$ & $0.42 \pm 0.29$ & $0.47 \pm 0.11$ & $0.42 \pm 0.11$ \\
\hline Grand Canyon No. 3 & $-0.14 \pm 1.2$ & $-0.47 \pm 0.33$ & $0.43 \pm 0.13$ & $0.57 \pm 0.13$ \\
\hline Grand Canyon No. 5 & $-2.2 \pm 1.2$ & $0.46 \pm 0.21$ & $0.76 \pm 0.13$ & $1.1 \pm 0.14$ \\
\hline Grand Canyon No. 6 & $-0.73 \pm 1.3$ & $0.91 \pm 0.33$ & $0.98 \pm 0.14$ & $0.90 \pm 0.14$ \\
\hline Grand Canyon No. 7 & $-0.0001 \pm 1.3$ & $1.1 \pm 0.35$ & $0.97 \pm 0.14$ & $0.91 \pm 0.15$ \\
\hline Grand $\mathrm{C}$ anyon No. & $-1.5 \pm 1$ & $0.33 \pm 0.30$ & $0.89 \pm 0.12$ & $0.61 \pm 0.12$ \\
\hline
\end{tabular}

1-Samples No. 2 and No. 4 were predominately water and were, therefore, not analyzed.

2-a-counting error. 
TABLE 6. Rio Puerco Background Samples

\begin{tabular}{|c|c|c|c|c|c|c|c|c|c|c|c|}
\hline STAKE & Distance & SAM & & THO & & & & & & UR & \\
\hline no. & METERS & TYPE & REP. & LEVEL ${ }^{\text {l }}$ & $\operatorname{ERROR}^{2}$ & LEVEL & EnRor & LEVEL & ERROR & LEUEL & ERROR \\
\hline 0 & 0 & 6 & 1 & -6.1 & 7.7 & 1.8 & .2 & .6 & .9 & -.8 & .90 \\
\hline 0 & 0 & 6 & 2 & 10.0 & 9.5 & 1.2 & .2 & 3.0 & 1.1 & .0 & $.9:$ \\
\hline 1 & 178 & 6 & 1 & -1.7 & 11.0 & 1.4 & .2 & 4.6 & 1.6 & -.5 & 1.20 \\
\hline$i$ & 178 & 6 & J & 11.0 & 14.0 & 1.7 & .2 & 2.1 & 1.6 & -1.3 & $1.1 *$ \\
\hline 11 & 1734 & 6 & 1 & 25.0 & 12,0 & 1.1 & .1 & 2.2 & 1.6 & .9 & 1.10 \\
\hline 11 & 1754 & 6 & 2 & -3.6 & 7.7 & 1.2 & .2 & 2.6 & .9 & .5 & $.9 n$ \\
\hline 21 & 3255 & 6 & 1 & 2.5 & 6.8 & 4.3 & .6 & .7 & .8 & -.1 & $.4 t$ \\
\hline 21 & 3255 & 6 & 2 & -12.3 & 9.2 & -.7 & 2.2 & -.5 & 1.2 & .4 & $.1 *$ \\
\hline 31 & 4704 & 6 & 1 & 3.7 & 9.0 & 1.3 & .2 & .0 & 1.0 & -.6 & 1.10 \\
\hline 31 & 1704 & 6 & 2 & 3.7 & 7.3 & .8 & .1 & 1.7 & .9 & 1.4 & .90 \\
\hline 11 & 6223 & 6 & 1 & 17.0 & 9.7 & 1.4 & .2 & 1.9 & 1.0 & .0 & .90 \\
\hline 11 & 6223 & 6 & 2 & 18.0 & 12.3 & .9 & .1 & 1.7 & 1.3 & -.7 & 1.08 \\
\hline 51 & 7827 & 6 & 1 & 8.9 & 13.0 & & & 1.5 & 1.6 & -2.6 & $1.1 *$ \\
\hline 61 & 9431 & 6 & 1 & -8.7 & 13.0 & & & 3.1 & 1.8 & 1.4 & 1.10 \\
\hline 71 & 11071 & 6 & 1 & -6.1 & 0.1 & 7.6 & 1.1 & 1.1 & .9 & -.1 & $.4 x$ \\
\hline B1 & 12728 & 6 & 1 & -12.9 & 8.3 & & & 1.1 & .9 & .9 & $.9 x$ \\
\hline 91 & 14238 & 6 & 1 & -16.9 & 9.8 & 1.9 & .2 & .6 & 1.1 & -.4 & 1.20 \\
\hline 101 & 15712 & 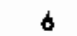 & ! & -1.7 & 11.0 & -4.2 & 2.2 & .4 & 1.1 & .5 & .5 \\
\hline 121 & 18807 & 6 & 1 & -9.8 & 9.1 & 2.0 & 1.7 & 1.0 & .9 & .2 & .40 \\
\hline 131 & 20427 & 6 & 1 & -1.7 & 9.2 & 1.1 & 1.5 & 2.5 & 1.1 & 1.8 & 1.0 \\
\hline 131 & 20427 & 6 & 2 & .0 & 12.0 & 1.4 & .2 & 4.2 & 1.7 & -3.8 & 1.10 \\
\hline 141 & 21942 & 6 & 1 & 11.0 & 9.2 & & & 1.1 & 1.1 & 1.1 & 1.00 \\
\hline 141 & 21942 & 6 & 2 & -10.9 & 13.0 & 3.5 & $t . \theta$ & 3.0 & 1.4 & 3.7 & 1,5 \\
\hline 141 & 21942 & 6 & 3 & 16.0 & 10.0 & 1.1 & 1.3 & 1.9 & 1.3 & 2.1 & 1.2 \\
\hline 151 & 23484 & $b$ & 1 & -8.9 & 9.8 & 1.0 & 1.4 & .2 & 1.3 & 1.6 & 1.1 \\
\hline 131 & 23484 & b & 2 & -1.1 & 7.3 & 2.4 & 1.3 & .8 & .9 & 3.6 & .9 \\
\hline 161 & 25162 & 6 & 1 & -8.7 & 9.5 & & & -.3 & 1.3 & 2.2 & $1.1 *$ \\
\hline 161 & 25162 & 6 & 2 & -8.6 & 7.5 & & & 2.6 & .8 & -.3 & .80 \\
\hline 171 & 26712 & 6 & $t$ & 25.0 & 13.0 & -.7 & 1.7 & 2.9 & 1.4 & 3.8 & 1.3 \\
\hline 171 & $267: 2$ & 6 & 2 & 1.8 & 9.9 & -2.1 & 1.8 & 1.7 & 1.3 & 4.8 & 1.3 \\
\hline 175 & 27315 & 6 & 1 & -6.1 & 8.7 & 1.2 & .2 & 1.0 & .9 & -.3 & 1.10 \\
\hline 173 & 27315 & 6 & 3 & 3.7 & 9.7 & 1.3 & .2 & 2.8 & 1.1 & .5 & $1.1 *$ \\
\hline $\mid \mathbf{B 1}$ & 28242 & 6 & $t$ & -8.6 & 7.7 & 4.7 & 1.7 & 2.6 & 1.0 & 2.1 & 1.0 \\
\hline 181 & 28242 & 6 & 2 & -4.9 & 12.0 & & & 2.3 & 1.6 & .2 & $1.1 *$ \\
\hline 181 & 28242 & 6 & 3 & -6.1 & 7.5 & 2.6 & 1.3 & 2.7 & 1.1 & 3.0 & 1.0 \\
\hline 191 & 29833 & 6 & 1 & -27.9 & 13,0 & 1.2 & 1.9 & .6 & 1.3 & -1.5 & $1.1 *$ \\
\hline 191 & 29033 & 6 & 2 & .0 & 9.4 & 1.7 & .2 & 1.3 & 1.0 & -.3 & $1.0 *$ \\
\hline 201 & 31239 & 1 & 1 & -17.9 & 11.0 & & & 6.3 & 1.6 & .2 & $1.1 *$ \\
\hline 201 & 31239 & 6 & 2 & 34.0 & 14.0 & 1.1 & .2 & 2.3 & 1.4 & 1.0 & $1.0 *$ \\
\hline 211 & 32695 & 6 & 1 & 5.3 & 12.0 & & & 2.3 & 1.1 & 1.0 & $1.0 *$ \\
\hline 221 & 34327 & 6 & 1 & -3.6 & 10.0 & 1.2 & .2 & 1.1 & 1.1 & .5 & $.9 *$ \\
\hline
\end{tabular}

1-Concentration in $\mathrm{pci} / \mathrm{g}$

2-Counting error. 
TABLE 6. (contd)

\begin{tabular}{|c|c|c|c|c|c|c|c|c|c|c|c|}
\hline \multirow{3}{*}{$\begin{array}{l}87 \text { AKE } \\
\text { No. } \\
23 i\end{array}$} & \multirow{3}{*}{$\begin{array}{l}\text { DISTANCE } \\
\text { HETERS } \\
\text { 35784 }\end{array}$} & \multirow{2}{*}{\multicolumn{2}{|c|}{$\begin{array}{l}\text { GAKPLE } \\
\text { TYPE REP, }\end{array}$}} & \multicolumn{2}{|c|}{ THDR I Uh } & \multicolumn{2}{|c|}{ RADIUA } & \multicolumn{2}{|c|}{ LEAD } & \multicolumn{2}{|c|}{ UAAUIUN } \\
\hline & & & & LEVEL & Enhon & LEVEL & ERR日R & LEVEL & ERROR & LEVEL & ERROR \\
\hline & & 6 & 1 & -15.9 & 10.0 & & & 1.9 & 1.1 & -1.2 & $1.1 \%$ \\
\hline 241 & 37477 & 6 & 1 & 12.0 & B. 0 & 1.1 & .1 & 2.3 & 1.0 & .0 & .94 \\
\hline 251 & 39005 & 6 & 2 & 3.7 & 9.5 & 2.8 & .3 & 1.4 & .9 & -1.5 & 1.0 \\
\hline 251 & 39005 & 6 & 3 & -8.6 & 8.3 & 1.0 & .1 & 1.0 & 1.0 &.- .4 & .94 \\
\hline 261 & 10551 & 6 & 1 & -8.6 & 9.5 & 1.1 & .1 & .0 & .8 & .4 & 1.00 \\
\hline 261 & 10551 & 6 & 2 & -7.3 & 0.2 & & & .8 & 1.1 & -1.3 & $.9 *$ \\
\hline 271 & 12184 & 6 & 1 & 11.0 & 9.7 & & & .6 & 1.1 & .0 & 1.00 \\
\hline 271 & 42184 & 6 & 2 & 3.5 & 13.0 & 1.5 & .2 & 2.1 & 1.7 & -1.1 & $1.0 *$ \\
\hline 281 & 13723 & 6 & 1 & 14.0 & 12.0 & 2.0 & 2.1 & 1.7 & 1.4 & 3.1 & 1.4 \\
\hline 281 & 43723 & 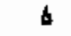 & 2 & 7.1 & 10.0 & 1.2 & 1.3 & 1.5 & 1.2 & 1.7 & 1.2 \\
\hline 291 & 45376 & 6 & 1 & .0 & 9.3 & 2.5 & 1.7 & 1.4 & 1.0 & 3.0 & 1.0 \\
\hline 311 & & 6 & $t$ & -13.9 & 8.0 & & & 2.5 & 1.1 & $1 . t$ & $.9 *$ \\
\hline 321 & & 6 & 1 & -1.9 & 11.9 & -.8 & 1.9 & 2.5 & 1.5 & 5.1 & 1.4 \\
\hline 331 & & 6 & 1 & 14.0 & 7.7 & & & 1.5 & 1.0 & -.3 & 1.00 \\
\hline 341 & & 6 & 1 & 3.5 & 10.9 & .0 & 1.8 & 2.3 & 1.5 & 4.2 & 1.3 \\
\hline 341 & & 6 & 2 & 9.9 & 10.0 & 1.1 & 2.1 & 2.3 & .9 & 4.5 & 1.2 \\
\hline 351 & & 6 & 1 & 7.1 & 10.6 & 2.7 & 1.9 & 1.7 & 1.5 & 5.7 & 1.4 \\
\hline 351 & & 6 & 2 & 7.4 & 9.0 & -.4 & 1.7 & 1.0 & 1.1 & 2.7 & 1.1 \\
\hline 361 & & 6 & 2 & -1.1 & 7.1 & -.4 & 1.8 & 1.2 & 1.0 & .2 & $.4 *$ \\
\hline 371 & & 6 & 1 & 8.7 & 9.0 & 1.3 & 2.0 & .7 & 1.0 & 1.2 & 1.0 \\
\hline 371 & & $b$ & 2 & 1.2 & 7.9 & -.0 & 1.9 & .6 & 1.0 & 3.2 & 1.0 \\
\hline 381 & & 6 & $t$ & 8.7 & 10.1 & 1.0 & 2.0 & 1.8 & 1.1 & 3.2 & 1.1 \\
\hline 381 & & 6 & 2 & -4.9 & 10.2 & 5.5 & 1.7 & .7 & 1.0 & 1.7 & 1.1 \\
\hline 391 & & 6 & 1 & -14.2 & 12.3 & .2 & 2.1 & .2 & 1.5 & 6.1 & 1.4 \\
\hline 391 & & 6 & 2 & 9.9 & 9.9 & 1.1 & 2.0 & 3.7 & 1.2 & 3.1 & 1.2 \\
\hline 101 & & 6 & 1 & -12.2 & 0.0 & .0 & 2.1 & 3.4 & 1.0 & .3 & .40 \\
\hline 101 & & 6 & 2 & -15.9 & 13.0 & & & 3.1 & 1.7 & 1.4 & 1.0 \\
\hline 411 & & 6 & $i$ & -1.1 & 9.2 & & & 1.9 & 1.0 & -.3 & $.9 *$ \\
\hline 421 & & 6 & 1 & B.7 & 5.4 & .4 & 1.3 & .8 & .8 & 1.2 & .8 \\
\hline 421 & & 6 & 2 & -4.9 & 7.6 & -.1 & 1.6 & 2.8 & 1.0 & 2.0 & 1.0 \\
\hline$|3|$ & & 6 & 1 & 23.0 & 11.3 & 3.1 & 2.0 & .2 & 1.5 & .1 & $.4 *$ \\
\hline 411 & & 6 & 1 & 15.9 & 13.1 & 7.7 & 1.6 & 1.3 & 1.3 & -.1 & $.5 *$ \\
\hline 141 & & 6 & 2 & -8.5 & 8.6 & -2.2 & 1.9 & .4 & 1.0 & .2 & $.4 *$ \\
\hline 431 & & 6 & I & 11.0 & 13.0 & & & .4 & 1.6 & 1.0 & $.9 *$ \\
\hline 451 & & 6 & 2 & -8.5 & 8.1 & & & 1.9 & 1.0 & -2.6 & 1.04 \\
\hline 481 & & 6 & 1 & 1.2 & 9.5 & -.7 & 1.8 & 1.0 & 1.0 & 3.1 & 1.1 \\
\hline 181 & & 6 & 2 & -5.2 & 10.5 & -.5 & 1.6 & .8 & 1.4 & 4.2 & 1.4 \\
\hline 491 & & 6 & 1 & .0 & 8.8 & 1.4 & 2.0 & 2.8 & 1.0 & 4.1 & 1.1 \\
\hline 491 & & 6 & 2 & -2.4 & 8.8 & -.8 & 1.8 & 1.4 & .9 & 2.5 & 1.0 \\
\hline & & $\bar{x}$ & $\hat{r}_{\mathrm{x}}$ & 0.89 & 11.2 & 1.3 & 2.0 & 1.7 & 1.2 & 1.1 & 2.0 \\
\hline
\end{tabular}


PRESENTATION OF RIO PUERCO SAMPLE DATA

All of the data that were obtained from the planar intrinsic germanium detectors were identified with only a sample number. These data were transmitted to Dr. Thomas Buhl of the New Mexico Environmental Improvement Division. The NMEID personnel associated the radionuclide concentration data with some descriptive information for each sampling site. This descriptive information included the stake number where the sample was collected and the sample type. The following identifications were given to the different sample types:

\begin{tabular}{ll}
$\frac{\text { Type }}{1}$ & \multicolumn{1}{c}{ Identification } \\
2 & First terrace \\
3 & Concentrated area \\
4 & Pool center \\
5 & Pool discharge point \\
6 & Background \\
7 & Core near stream \\
8 & Core near cut bank \\
9 & Associated salts
\end{tabular}

Also included in the information submitted by the NMEID was an indication of the number of replicates that were taken for each of the sample types at a given stake. All of this information was transferred to PNL on computer cards. A total of 1191 individual computer cards (data for individual samples) was received. The data from this deck of computer cards for the concentrations of the four radionuclides of interest and the associated counting errors are given in Appendix B. These data are listed for ${ }^{230}$ Th (listed as "THORIUM"), ${ }^{226}$ Ra ("RADIUM"), $210 \mathrm{~Pb}$ ("LEAD"), and either ${ }^{235} \mathrm{~J}$ or $238 \mathrm{U}$ ("URANIUM"). Those uranium concentrations which have an asterisk (*) printed beside the "ERROR" value are reported as ${ }^{235} \mathrm{U}$. All other uranium values are reported as ${ }^{238} \mathrm{U}$.

These data were statistically analyzed on the PNL biometrics computer, a POP-11/70. Concentrations of individual radionuclides for indiviaual sample types were plotted as a function of distance downstream from the point of tailings dam breaching or as a function of stake number downstream. All the 
replicates of each type of sample collected at each stake were averaged using a weighted average where the weights were proportional to the inverse of the counting error squared. This results in data with small counting errors being more important than data with large counting errors. The extreme heterogeneity and variability of the data were evident from these plots. Therefore, the smoothing function previously described in the statistical methods section was applied to provide graphical presentations that were more easily visualized and understood. The smoothed averages for ${ }^{210} \mathrm{~Pb},{ }^{226} \mathrm{Ra}$, and ${ }^{238} \mathrm{U}$ were plotted as a funcion of distance downstream from the tailings pond but displayed no significant variability from typical plots of "background" concentration versus distance for these three radionuclides. Therefore, no plots of concentration versus distance for these three radionuclides have been included in this report. However, the plots of ${ }^{230}$ Th concentration versus distance do display a downstream variability greater than typical background levels. The plots of the ${ }^{230}$ Th smoothed averages, which also contain the background sample concentrations, are given in Appendix C. Figure 4 is an example of these plots showing ${ }^{230}$ Th concentration versus distance.

All distance measurements start at stake number zero which is located at the point where the spill entered the arroyo. Large U.S.G.S. sector maps were provided by United Nuclear Corporation (UNC) with stake locations added to the maps for the first 305 stakes, which corresponds to a distance that is just beyond the downstream side of the city of Gallup. (These maps are presented in Appendix D.) It was possible to measure the actual distances between the stakes from these maps, and these distances were used where available. For samples collected beyond the range of these maps, the stake numbers were used. It was noticed that the distance between the stakes when measured along the watercourse of the arroyo varies substantially; this is due to the fact that stake distance was measured at the top of the arroyo cut. Because the arroyo often twists and turns several times between two stakes, these two measures of distance do not agree very well.

As shown in Figure 4 and in the remaining plots in Appendix $C$, the smoothed averages for thorium strongly suggest a periodic trend with a wavelength of approximately $2.5 \mathrm{~km}$. This possible periodicity suggests that 


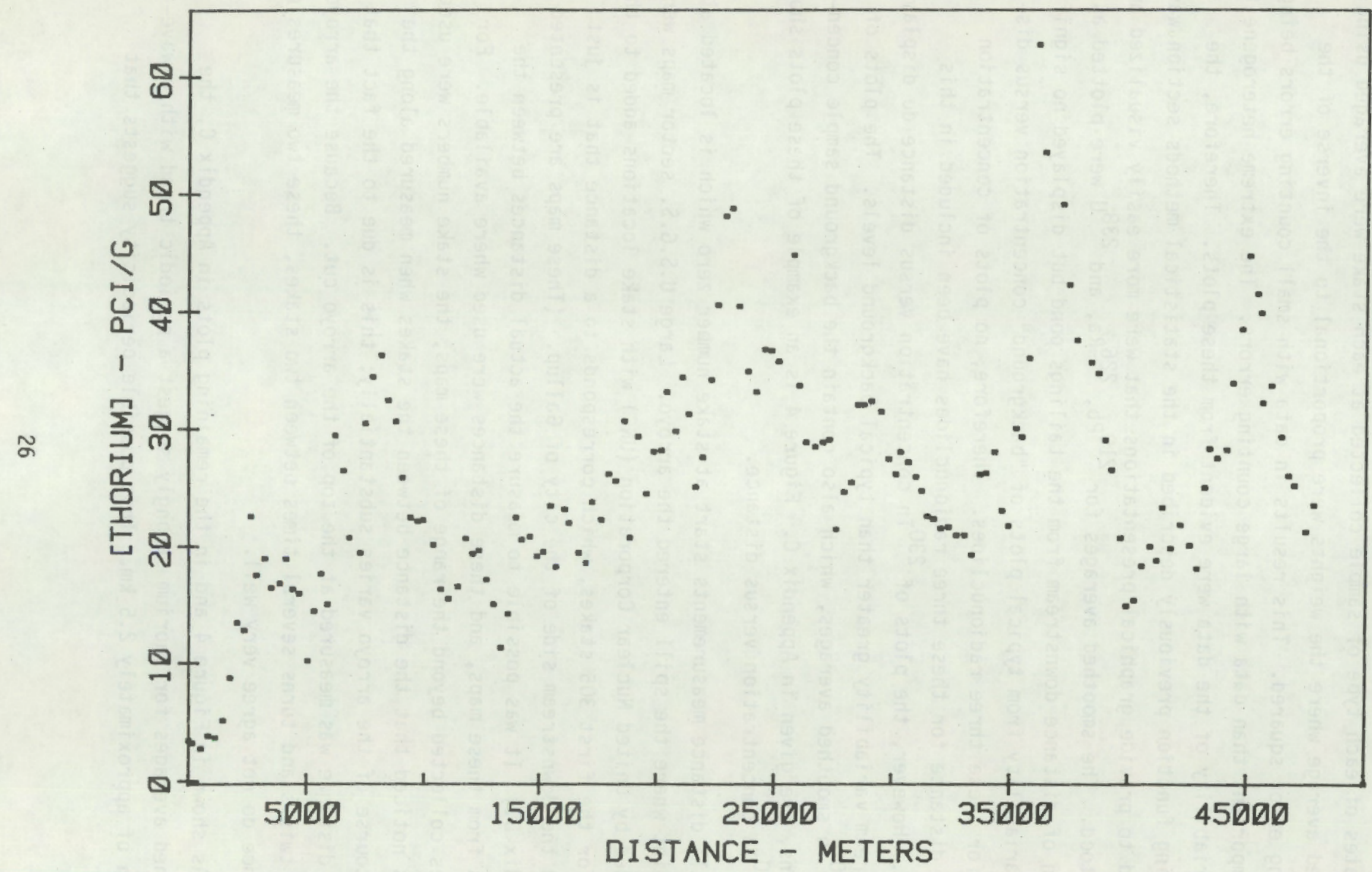

FIGURE 4. ${ }^{230}$ Th Concentration Versus Distance, First Terrace Samples 
extensive data interpretation not be performed until further statistical testing is performed. Such testing was beyond the scope of this research investigation. Therefore, our knowledge that a periodic structure is likely mandates some adjustments in any further data analysis. Time series theory tells us that in order to average out a periodic trend we must average over a minimum of two periods. The possible trend in the thorium data seems to have a wavelength of around $2.5 \mathrm{~km}$. To use any distance less than $5 \mathrm{~km}$ for determining average ${ }^{230}$ Th concentrations in a statistically rigorous analysis will require a formal time series analysis of the data.

The plots of the ${ }^{230}$ Th concentration versus distance may be used as a simple approach for determining those areas showing levels greater than the cleanup criteria of $30 \mathrm{pCi} / \mathrm{g}$. Table 7 lists the 415 individual samples which equal or exceed the $30 \mathrm{pCi} / \mathrm{g}^{230}$ Th cleanup criteria. Approximately 250 of these samples were from the first and second terrace areas. The remaining 165 samples were obtained specifically from areas expected to be "hot spots" along the Rio Puerco.

\section{EVALUATION OF GRID SAMPLING DATA}

The New Mexico Environmental Improvement Division personnel selected two sampling sites within the Rio Puerco arroyo which were subdivided into grids of 25-ft intervals and sampled on these intervals. At both sites additional samples were obtained at the corners and in the center of one or more 5-ft squares. The purpose of these samplings and the subsequent analysis of the data obtained from these samples was to evaluate the localized spatial variability at two typical sediment sampling locations. (These two sampling locations were both flat sandbars.) The sampling patterns for each of these sites are given in Figures 5 and 6 . The radionuclide concentration data for these sites are given in Appendix E.

A rigorous spatial analysis of these studies was not performed; however, the data were reviewed. The examination of these data relative to the sampling locations does not suggest any spatial pattern. At a few selected sampling locations within each of these grids replicate samples were obtained (the samples collected from the $5-\mathrm{ft}$ squares). The variability of the data from 
TABLE 7. Rio Puerco Samples Having 230 Th Concentrations $>30 \mathrm{pCi} / \mathrm{g}$

\begin{tabular}{|c|c|c|c|c|c|c|c|c|c|c|c|}
\hline \multirow{2}{*}{$\begin{array}{l}\text { STAKE } \\
\text { WO. }\end{array}$} & \multirow{2}{*}{$\begin{array}{l}\text { DISTAMCE } \\
\text { WE TERA }\end{array}$} & \multicolumn{2}{|c|}{ SAMPLE } & \multicolumn{2}{|c|}{ THORIUK } & \multicolumn{2}{|c|}{ RADIUM } & \multicolumn{2}{|c|}{ LEAD } & \multicolumn{2}{|c|}{ URAMIUH } \\
\hline & & TYPE & REP. & LEVEL ${ }^{1}$ & ERROR $^{2}$ & LEVEL & ERROR & LEUEL & ERROR & LEUEL & ERROR \\
\hline 0 & 0 & 7 & 2 & 130.0 & 22.0 & 2.3 & .3 & 19.0 & 2.7 & 1.4 & $1.2 *$ \\
\hline 0 & 0 & 8 & 3 & 157.0 & 22.0 & 6.8 & .7 & 59.0 & 3.4 & 4.0 & $1.4 *$ \\
\hline 1 & 178 & 7 & 1 & 57.0 & 13.0 & 2.2 & .2 & 8.5 & 1.5 & -.1 & $1.1 *$ \\
\hline 1 & 178 & 7 & 2 & 35.0 & 15.0 & 1.2 & .2 & 6.3 & 1.7 & -.5 & $1.1 \%$ \\
\hline 1 & 178 & 7 & 3 & 51.0 & 15.0 & 1.8 & .2 & 9.0 & 1.9 & -1.3 & $1.2 *$ \\
\hline 3 & 521 & 4 & 1 & 100.0 & 17.0 & 1.4 & .2 & 19.0 & 2.1 & .4 & $1.0 *$ \\
\hline 3 & 521 & 4 & 2 & 200.0 & 19.0 & & & 11.0 & 1.8 & .7 & $1.0 *$ \\
\hline 3 & 521 & 9 & 1 & 62.0 & 16.0 & .0 & .4 & 9.0 & 2.1 & 1.2 & 1.2 * \\
\hline 7 & 1147 & 2 & 1 & 89.0 & 19.0 & 2.8 & .3 & 7.1 & 2.0 & -.7 & $1.2 *$ \\
\hline 7 & 1147 & 1 & 1 & 200.0 & 21.0 & 3.8 & .4 & 44.0 & 3.0 & .2 & $1.4 *$ \\
\hline 9 & 1471 & 2 & 1 & 49.0 & 13.0 & 1.4 & .2 & 3.3 & 1.3 & .9 & $1.0 *$ \\
\hline 9 & 1471 & 2 & 2 & 36.0 & 12.0 & 0.6 & .2 & 9.9 & 1.5 & .5 & $1.0 \%$ \\
\hline 11 & 1754 & 1 & 1 & 34.0 & 15.0 & 1.9 & .2 & 1.9 & 1.8 & -.5 & $1.2 *$ \\
\hline 13 & 2073 & 1 & 1 & 84.0 & 15.0 & 2.7 & .3 & 24.0 & 2.1 & .2 & $1.2 *$ \\
\hline 17 & 2657 & 1 & 2 & 92.0 & 15.0 & 1.6 & .2 & 16.0 & 1.8 & -.1 & $1.2 *$ \\
\hline 19 & 2897 & 1 & 1 & 110.0 & 21.0 & 2.7 & .3 & 30.0 & 3.3 & 1.6 & $1.3 *$ \\
\hline 19 & 2897 & 3 & 1 & 270.0 & 22.0 & 1.1 & .2 & 11.0 & 1.6 & 2.0 & $1.3 *$ \\
\hline 21 & 3255 & 1 & 2 & 44.3 & 16.1 & .6 & 2.2 & 11.0 & 2.3 & 7.5 & 1.0 \\
\hline 21 & 3255 & 8 & 1 & 54.0 & 12.0 & .1 & 2.0 & 12.0 & 1.6 & 1.6 & 1.1 \\
\hline 27 & 1140 & 1 & 2 & 30.1 & 15.5 & 1.5 & 1.8 & 7.7 & 1.7 & 6.8 & 1.6 \\
\hline 27 & 4140 & 9 & 1 & 85.0 & 14.0 & & & 15.0 & 1.8 & .7 & $1.0 *$ \\
\hline 31 & 4704 & 7 & 2 & 47.8 & 15.9 & .0 & 2.1 & 10.7 & 2.0 & 6.5 & 1.6 \\
\hline 31 & 4704 & 7 & 3 & 14.6 & 10.1 & 1.6 & 1.9 & 6.3 & 1.3 & 3.1 & 1.1 \\
\hline 31 & 4704 & $B$ & 2 & 34.7 & 11.8 & 2.6 & 2.0 & 5.6 & 1.2 & 4.0 & 1.0 \\
\hline 35 & 5338 & 1 & 2 & 42.0 & 16.0 & 1.7 & .2 & .0 & 3.5 & $=.9$ & 2.30 \\
\hline 37 & 5616 & 3 & 1 & 140.0 & 24.0 & 2.2 & .3 & 26.0 & 3.0 & .2 & $1.2 *$ \\
\hline 37 & 5616 & 3 & 2 & 36.0 & 12.0 & 1.4 & .2 & 5.5 & 1.2 & .7 & $.9 *$ \\
\hline 39 & 5899 & 3 & 1 & 34.0 & 16.0 & 1.7 & .2 & 8.6 & 2.3 & -.1 & $1.1 *$ \\
\hline 41 & 6223 & 1 & 1 & 67.0 & 14.0 & 1.0 & .1 & 5.9 & 2.0 & .8 & .90 \\
\hline 41 & 6223 & 1 & 2 & 77.0 & 13.0 & & & 4.4 & 1.2 & .4 & $1.0 *$ \\
\hline 41 & 6223 & 7 & 1 & 51.0 & 16.0 & 1.2 & .2 & 8.4 & 1.8 & 1.2 & $1.0 *$ \\
\hline 47 & 7309 & 1 & 1 & 30.9 & 9.0 & -2.7 & 1.7 & 4.1 & 1.2 & 4.5 & 1.0 \\
\hline 47 & 7309 & 2 & 1 & 45.9 & 10.9 & 1.8 & 1.7 & 3.1 & 1.1 & 2.8 & 1.0 \\
\hline 49 & 7525 & 1 & 1 & 42.5 & 13.7 & -.1 & 2.0 & 6.7 & 1.8 & 6.3 & 1.3 \\
\hline 49 & 7525 & 1 & 2 & 103.0 & 19.0 & & & 10.0 & 2.0 & 2.0 & $1.2 *$ \\
\hline 51 & 7872 & 1 & 1 & 79.0 & 16.0 & 1.8 & 2.9 & 6.6 & 1.2 & 10.0 & 1.7 \\
\hline 51 & 7827 & 1 & 2 & 31.0 & 11.0 & 1.1 & 1.8 & 3.0 & 1.0 & 3.0 & 1.1 \\
\hline 51 & 7827 & 7 & 2 & 76.0 & 14.0 & 1.9 & 1.7 & 8.1 & 1.4 & .7 & .90 \\
\hline 53 & 8163 & 1 & 1 & 64.0 & 13.0 & 3.3 & 2.0 & 9.2 & 1.4 & 4.5 & 1.3 \\
\hline 53 & 8163 & 4 & 1 & 52.0 & 13.0 & & & 9.0 & 1.5 & 1.1 & $1.0 *$ \\
\hline 55 & 8473 & 1 & 2 & 83.0 & 18.0 & 1.1 & .2 & 6.1 & 1.9 & 1.0 & $1.1 \%$ \\
\hline
\end{tabular}

l-Concentration in $\mathrm{pCi} / \mathrm{g}$.

2-Counting error. 
TABLE 7. (contd)

\begin{tabular}{|c|c|c|c|c|c|c|c|c|c|c|c|}
\hline STAKE & JISTAMCE & sam & PLE & THO & & & & & & URA & \\
\hline Mo. & HETERS & TYPE & REP. & LEVEL & ERROR & LEVEL & ERROR & LEVEL & ERROR & LEUEL & ERROR \\
\hline 57 & 8002 & 1 & 2 & 53.0 & 13.0 &.- .5 & 2.2 & 8.8 & 1.3 & 6.6 & 1.3 \\
\hline 57 & 8802 & 3 & 1 & 112.0 & 21.0 & 7.2 & 2.3 & 13.0 & 2.5 & .6 & $1.1 *$ \\
\hline 61 & 9431 & 2 & 2 & 35.0 & 14.0 & -1.0 & 1.8 & 2.7 & 1.6 & 4.6 & 1.3 \\
\hline 61 & 9431 & 4 & 1 & 73.0 & 18.0 & 1.3 & .2 & 10.0 & 2.2 & -2.1 & $1.3 *$ \\
\hline 63 & 9721 & 1 & 2 & 35.0 & 11.0 & 3.3 & 1.7 & 4.3 & 1.1 & .7 & $.9 *$ \\
\hline 63 & 9721 & 2 & 1 & 33.7 & 13.6 & -2.8 & 2.2 & 7.1 & 1.8 & $? .2$ & 1.4 \\
\hline 63 & 9721 & 2 & 2 & 39.6 & 12.6 & .1 & 2.0 & 7.2 & 1.3 & 6.2 & 1.3 \\
\hline 65 & 9983 & 4 & 1 & 69.3 & 13.9 & -5.5 & 2.6 & 9.2 & 1.4 & .8 & $.5 *$ \\
\hline 73 & 11498 & 2 & 1 & 37.0 & 12.0 & 4.7 & 1.6 & 7.4 & 1.3 & -.0 & .5 \\
\hline 73 & 11498 & 4 & 1 & 170.0 & 23.0 & -13.9 & 3.7 & 12.0 & 2.3 & 1.9 & .7 \\
\hline 75 & 11829 & 1 & 1 & 46.0 & 11.0 & 1.0 & .1 & 5.1 & 1.3 & .5 & $.9 \%$ \\
\hline 75 & 11829 & 4 & 1 & 35.0 & 10.0 & 1.0 & .1 & 5.9 & 1.3 & .4 & $.9 *$ \\
\hline 79 & 12385 & 2 & 2 & 70.6 & 11.4 & -1.1 & 2.1 & 8.0 & 1.4 & .1 & $.5 *$ \\
\hline 79 & 12385 & 4 & 1 & 127.0 & 20.0 & & & 9.2 & 2.1 & 2.9 & $1.1 *$ \\
\hline 81 & 12728 & 1 & 1 & 96.0 & 20.0 & & & 6.1 & 2.1 & .8 & $1.1 *$ \\
\hline B1 & $1272 B$ & 7 & 2 & 62.0 & 15.5 & -16.3 & 3.0 & 5.2 & 1.6 & 1.4 & $.5 *$ \\
\hline 83 & 13021 & 4 & 1 & 58.0 & 12.0 & .8 & .2 & 6.5 & 1.2 & 1.1 & $.9 *$ \\
\hline 85 & 13362 & 4 & 1 & 69.0 & 18.0 & 4.3 & 2.0 & 11.1 & 2.1 & 2.3 & 1.30 \\
\hline 87 & 13643 & 3 & 1 & 85.0 & 18.0 & .9 & .2 & 4.2 & 2.1 & .0 & 1.10 \\
\hline 87 & 13643 & 1 & 1 & 33.0 & 12.0 & 5.0 & 2.0 & 4.1 & 1.2 & .0 & $.9 *$ \\
\hline 87 & 13643 & 4 & 2 & 32.1 & 12.0 & -.0 & 2.4 & 3.0 & 1.2 & .5 & .40 \\
\hline 89 & 13967 & 1 & 1 & 47.0 & 10.0 & -5.4 & 2.3 & 4.0 & 1.1 & .6 & .4 \\
\hline 89 & 13967 & 4 & 1 & 35.4 & 14.3 & 9.5 & 1.4 & 1.6 & 1.6 & -.1 & $.6 *$ \\
\hline 91 & 14238 & 2 & 1 & 90.3 & 16.3 & -2.0 & 2.7 & 7.7 & 1.8 & .7 & $.5 *$ \\
\hline 93 & 14586 & 1 & 1 & 40.8 & 11.6 & -11.8 & 2.8 & 6.6 & 1.3 & 1.1 & $.5 *$ \\
\hline 93 & 14586 & 2 & 1 & 88.6 & 16.8 & -8.0 & 2.7 & 7.1 & 1.8 & 1.1 & $.6 *$ \\
\hline 95 & 14874 & 4 & 2 & 139.0 & 17.0 & 12.0 & 2.3 & 6.2 & 1.5 & .9 & $1.1 \%$ \\
\hline 97 & 15130 & 1 & 2 & 49.6 & 15.0 & 1.3 & 1.7 & 3.3 & 1.8 & .1 & $.5 *$ \\
\hline 99 & 15448 & 1 & 1 & 34.0 & 12.0 & 2.8 & 2.0 & 1.0 & 1.5 & .3 & .6 \\
\hline 99 & 15448 & 1 & 2 & 36.0 & 11.0 & -9.2 & 2.6 & 2.1 & 1.1 & .9 & $.5 *$ \\
\hline 99 & 15448 & 4 & 1 & 38.0 & 11.0 & 8.0 & 2.0 & 4.8 & 1.3 & .5 & $1.0 *$ \\
\hline 99 & 15448 & 4 & 2 & 110.0 & 19.0 & & & .0 & 2.1 & 1.0 & $1.1 *$ \\
\hline 99 & 15448 & 1 & 4 & 79.0 & 15.0 & 2.5 & .3 & 15.0 & 1.8 & .7 & $1.3 *$ \\
\hline 101 & 15712 & 4 & 1 & 35.0 & 14.0 & 1.8 & .2 & 3.1 & 1.9 & -.3 & $1.2 *$ \\
\hline 103 & 16062 & 1 & 1 & 38.0 & 11.0 & -1.4 & 2.2 & 6.8 & 1.3 & .5 & $.5 *$ \\
\hline 103 & 16062 & 2 & 1 & 51.0 & 15.0 & -6.7 & 2.7 & b.1 & 1.7 & 1.0 & $.5 *$ \\
\hline 105 & 16264 & 9 & 1 & 210.0 & 20.0 & 2.9 & .3 & 8.8 & 1.6 & .4 & $1.1 *$ \\
\hline 107 & 16689 & 2 & 1 & 36.0 & 11.0 & -.7 & .7 & 1.6 & 1.0 & .2 & $.5 *$ \\
\hline 107 & 16609 & 4 & 1 & 37.0 & 10.0 & -7.3 & 2.3 & 2.3 & 1.1 & .6 & . 4* \\
\hline 109 & 17015 & 2 & 1 & 70.8 & 14.8 & -4.3 & 2.1 & 2.3 & 1.3 & .6 & $.5 *$ \\
\hline 111 & 17293 & 1 & 1 & 39.0 & 13.0 & -1.7 & 2.0 & 2.3 & 1.6 & .3 & $.5 *$ \\
\hline
\end{tabular}


TABLE 7. (contd)

\begin{tabular}{|c|c|c|c|c|c|c|c|c|c|c|c|}
\hline \multirow{3}{*}{$\begin{array}{l}\text { STAKE } \\
\text { MO. }\end{array}$} & \multirow{2}{*}{$\begin{array}{l}\text { DISTAWCE } \\
\text { METERS }\end{array}$} & \multicolumn{2}{|c|}{ SAMPLE } & \multicolumn{2}{|c|}{ THORIUK } & \multicolumn{2}{|c|}{ RADIUK } & \multicolumn{2}{|c|}{ LEAD } & \multicolumn{2}{|c|}{ URAUluy } \\
\hline & & TYPE & REP. & LEVEL & ERROR & LEVEL & ERROR & LEVEL & ERROR & LEVEL & ERROR \\
\hline & 17293 & 2 & 1 & 40.0 & 11.0 & -6.0 & 2.7 & 7.7 & 1.2 & 1.0 & $.4 *$ \\
\hline 111 & 17293 & 7 & 1 & 48.0 & 13.0 & -.2 & .4 & 2.1 & 1.2 & -2.7 & .40 \\
\hline 113 & 17663 & 2 & 1 & 50.8 & 11.0 & -2.3 & 2.2 & 6.5 & 1.3 & .5 & $.5 *$ \\
\hline 115 & 17965 & 1 & 1 & 38.0 & 10.0 & & & 4.1 & 1.1 & 1.1 & $1.0 *$ \\
\hline 115 & 17965 & 2 & 1 & 30.0 & 9.3 & .8 & .2 & -.3 & 1.0 & -.1 & $.9 *$ \\
\hline 117 & 18277 & 2 & 1 & 67.3 & 13.7 & -18.5 & 3.0 & 4.2 & 1.6 & 1.7 & $.5 *$ \\
\hline 119 & 18630 & 1 & 1 & 45.8 & 10.3 & 5.1 & 1.4 & 2.3 & 1.2 & -.0 & $.3 *$ \\
\hline 121 & 18887 & 2 & 1 & 12.1 & 11.3 & -1.2 & 2.2 & 3.2 & 1.1 & .5 & $.4 *$ \\
\hline 123 & 19209 & 1 & 1 & 54.9 & 13.1 & -1.1 & 2.2 & 4.2 & 1.4 & .5 & $.5 *$ \\
\hline 123 & 19209 & 2 & 1 & 34.6 & 9.6 & 2.2 & 2.0 & 1.0 & 1.0 & .2 & $.4 *$ \\
\hline 123 & 19209 & 2 & 2 & 43.3 & 11.8 & 7.6 & 1.2 & 7.2 & 1.2 & -.1 & $.4 *$ \\
\hline 125 & 19555 & 2 & 1 & 71.0 & 13.0 & 7.7 & 1.8 & 4.0 & 1.2 & -.6 & $1.1 *$ \\
\hline 127 & 19879 & 1 & 1 & 100.9 & 18.3 & -9.5 & 3.4 & 4.3 & 1.8 & 1.7 & $.6 *$ \\
\hline 127 & 19879 & 2 & 1 & 82.9 & 12.8 & -.4 & 2.5 & 6.6 & 1.3 & .5 & $.5 *$ \\
\hline 127 & 19879 & 2 & 2 & 84.2 & 14.7 & -5.5 & 2.6 & 7.6 & 1.4 & .8 & $.5 *$ \\
\hline 129 & 20189 & 2 & 1 & 65.0 & 17.0 & 1.3 & .2 & 4.0 & 1.8 & -.7 & $1.2 *$ \\
\hline 129 & 20189 & 2 & 2 & 37.0 & 13.0 & 1.0 & .1 & 1.9 & 1.7 & .8 & $.9 *$ \\
\hline 131 & 20427 & 1 & 1 & 48.0 & 13.0 & .4 & .1 & 4.0 & 1.6 & .6 & $1.0 *$ \\
\hline 131 & 20427 & 2 & 1 & 30.0 & 15.0 & 1.0 & .1 & 3.8 & 1.6 & .6 & $1.0 *$ \\
\hline 131 & 20427 & 4 & 1 & 58.0 & 13.0 & .7 & .1 & 2.2 & 1.2 & .0 & $1.0 *$ \\
\hline 131 & 20427 & 4 & 2 & 44.0 & 14.0 & 1.5 & .2 & 4.2 & 1.8 & .0 & $1.1 *$ \\
\hline 131 & 20427 & 4 & 3 & 120.0 & 16.0 & 8.5 & 2.1 & 9.1 & 1.6 & 11.0 & 1.5 \\
\hline 131 & 20427 & 8 & 2 & 14.0 & 12.0 & 2.6 & 1.4 & 1.8 & 1.3 & 5.1 & 1.3 \\
\hline 133 & 20789 & 1 & 2 & 30.0 & 10.0 & 3.7 & 1.6 & 2.1 & 1.3 & 3.6 & 1.2 \\
\hline 133 & 20709 & 2 & 2 & 100.0 & 16.0 & 16.0 & 1.7 & 5.4 & 1.4 & 1.3 & $1.0 *$ \\
\hline 135 & 21077 & 1 & 1 & 37.0 & 14.0 & 3.2 & 1.6 & 1.0 & 1.7 & .8 & $.9 *$ \\
\hline 135 & 21077 & 1 & 2 & 67.0 & 18.0 & & & 4.0 & 1.7 & .6 & $1.1 *$ \\
\hline 137 & 21635 & 2 & 1 & 55.0 & 16.0 & .6 & 1.6 & 2.8 & 1.5 & 2.8 & 1.4 \\
\hline 139 & 21644 & 2 & 1 & 35.0 & 11.0 & 1.4 & 1.7 & 1.7 & 1.5 & 4.1 & 1.2 \\
\hline 141 & 21942 & 1 & 1 & 35.0 & 12.0 & 1.4 & 1.5 & 5.4 & 1.2 & 6.7 & 1.2 \\
\hline 141 & 21942 & 8 & 1 & 44.0 & 11.0 & 4.9 & 1.7 & 6.2 & 1.2 & 6.3 & 1.2 \\
\hline 141 & 21942 & 8 & 2 & 30.0 & 12.0 & 4.3 & 1.7 & 2.5 & 1.3 & 3.8 & 1.1 \\
\hline 143 & 22295 & 1 & 1 & 66.0 & 12.0 & 2.5 & 1.6 & 1.6 & 1.2 & 6.7 & 1.3 \\
\hline 143 & 22295 & 1 & 2 & 35.0 & 15.0 & 5.5 & 1.6 & 1.5 & 1.4 & 2.7 & 1.3 \\
\hline 143 & 22295 & 2 & 1 & 48.0 & 11.0 & 8.6 & 1.8 & 3.3 & 1.2 & 4.8 & 1.1 \\
\hline 145 & 22583 & 1 & 1 & 14.0 & 14.0 & & & 5.0 & 1.7 & .6 & $.9 *$ \\
\hline 145 & 22583 & 1 & 2 & 80.0 & 16.0 & 5.5 & 1.8 & 2.9 & 1.8 & .4 & $1.2 \%$ \\
\hline 145 & 22503 & 2 & 3 & 31.0 & 10.0 & 3.8 & 1.5 & 3.4 & 1.0 & -.4 & 1.0 \\
\hline 147 & 22912 & 1 & 1 & 55.0 & 15.0 & .8 & 1.9 & 2.7 & 1.5 & 5.3 & 1.4 \\
\hline 147 & 22912 & 1 & 2 & 03.0 & 13.0 & . & 1.7 & 5.0 & 1.3 & 7.1 & 1.4 \\
\hline 147 & 22912 & 2 & 1 & 112.0 & 20.0 & 10.0 & 2.4 & 9.6 & 2.4 & 2.0 & $1.3 *$ \\
\hline
\end{tabular}


TABLE 7. (contd)

\begin{tabular}{|c|c|c|c|c|c|c|c|c|c|c|c|}
\hline STAKE & DISTAMCE & san & & Tho & & & & & & URA & \\
\hline No. & METERS & TYPE & REP. & LEVEL & ERROR & LE VEL & ERROR & LEVEL & ERROR & LEVEL & ERROR \\
\hline 149 & 23186 & 1 & 2 & 90.0 & 15.0 & 4.8 & 1.7 & 5.8 & 1.3 & 7.9 & 1.4 \\
\hline 151 & 23484 & 1 & 2 & 60.0 & 16.0 & -2.2 & 2.3 & 2.3 & 1.6 & 8.5 & 1.7 \\
\hline 151 & 23484 & 4 & 1 & 147.0 & 23.0 & -8.3 & 3.0 & 20.0 & 2.8 & 18.0 & 2.3 \\
\hline 153 & 23856 & 2 & 2 & 30.0 & 13.0 & -.3 & 1.9 & 4.0 & 1.4 & 2.9 & 1.3 \\
\hline 155 & 24209 & 1 & 1 & 77.0 & 13.0 & 6.6 & 1.9 & 3.4 & 1.2 & -.8 & $1.1 \%$ \\
\hline 155 & 24209 & 2 & 1 & 42.0 & 12.0 & 1.9 & 1.5 & 1.4 & 1.2 & -.3 & $1.0 *$ \\
\hline 157 & 24569 & 1 & 1 & 53.1 & 14.2 & 7.5 & 2.0 & 6.5 & 1.8 & .0 & $.5 *$ \\
\hline 157 & 24569 & 1 & 2 & 11.0 & 15.0 & 4.8 & 2.0 & 5.4 & 1.7 & .1 & $.6 *$ \\
\hline 139 & 24857 & 1 & 2 & 43.0 & 10.0 & 6.8 & 1.1 & 3.2 & 1.1 & -.1 & $.4 *$ \\
\hline 159 & 24857 & 2 & 1 & 14.0 & 11.0 & -7.8 & 2.4 & 1.5 & 1.1 & .8 & .40 \\
\hline 159 & 24857 & 4 & 1 & 237.0 & 25.0 & & & 16.0 & 2.6 & 1.4 & $1.3 *$ \\
\hline 159 & 24857 & 5 & 2 & 74.0 & 20.0 & & & 6.1 & 2.3 & .2 & $1.0 *$ \\
\hline 161 & 25162 & 1 & 1 & 35.0 & 16.0 & -1.6 & 2.4 & 4.0 & 1.7 & .5 & $.5 *$ \\
\hline 161 & 25162 & 1 & 3 & 35.0 & 11.0 & 1.0 & 2.0 & 5.1 & 1.1 & .2 & $.4 *$ \\
\hline 161 & 25162 & 1 & 1 & 152.0 & 19.0 & 9.0 & 2.3 & 0.1 & 1.7 & 11.0 & 1.6 \\
\hline 161 & 25162 & 5 & 1 & 74.0 & 17.0 & 3.7 & 1.6 & 3.2 & 1.7 & 6.8 & 1.6 \\
\hline 161 & 25162 & 9 & 1 & 226.8 & 25.4 & -13.6 & 4.0 & 22.3 & 2.9 & 2.1 & .70 \\
\hline 163 & 25445 & 2 & 2 & 32.0 & 13.0 & 1.1 & .1 & 1.7 & 1.7 & .2 & $1.0 *$ \\
\hline 163 & 25445 & 4 & 1 & 120.0 & 18.0 & 2.2 & .3 & 12.0 & 1.7 & -.1 & $1.0 *$ \\
\hline 165 & 25789 & 1 & 1 & 72.0 & 17.0 & 3.5 & 1.6 & 4.0 & 1.6 & 6.1 & 1.6 \\
\hline 167 & 26036 & 2 & 1 & 46.0 & 16.0 & 2.9 & 1.8 & 5.6 & 1.6 & 4.8 & 1.5 \\
\hline 169 & 26326 & 9 & 1 & 34.0 & 16.0 & 4.9 & 1.7 & 1.0 & 1.6 & -.3 & $1.0 *$ \\
\hline 171 & 26712 & 1 & 2 & 41.0 & 13.0 & 3.2 & 1.7 & 4.2 & 1.4 & 5.1 & 1.4 \\
\hline 171 & 26712 & 2 & 2 & 35.0 & 13.0 & 2.9 & 1.6 & .6 & 1.4 & 6.3 & 1.3 \\
\hline 171 & 26712 & 8 & 1 & 33.0 & 10.0 & 6.3 & 1.8 & 1.1 & 1.0 & 3.0 & 1.0 \\
\hline 173 & 27029 & 1 & 1 & 53.0 & 11.0 & 4.6 & 1.7 & 3.1 & 1.2 & 6.6 & 1.2 \\
\hline 173 & 27029 & 4 & 1 & 48.0 & 17.0 & 7.8 & 2.2 & 6.3 & 1.8 & .2 & $1.3 *$ \\
\hline 175 & 27315 & 1 & 2 & 32.0 & 11.0 & 3.6 & 1.7 & 2.5 & 1.1 & .9 & $.9 *$ \\
\hline 173 & 27315 & 2 & 1 & 105.0 & 17.0 & & & 8.3 & 1.5 & 2.0 & $1.0 *$ \\
\hline 175 & 27315 & 3 & 1 & 54.0 & 12.0 & 1.3 & .4 & 2.5 & 1.1 & -.1 & $1.0 *$ \\
\hline 175 & 27313 & 4 & 1 & 111.6 & 21.6 & .8 & 2.4 & 13.4 & 2.3 & .5 & $.7 \%$ \\
\hline 175 & 27315 & 5 & 1 & 39.0 & 13.0 & 3.7 & .6 & 5.9 & 1.8 & 3.3 & $1.0 *$ \\
\hline 177 & 27608 & 3 & 1 & 10.0 & 12.0 & 2.9 & .5 & 5.1 & 1.4 & -.6 & $1.0 *$ \\
\hline 177 & 27608 & 1 & 1 & 60.0 & 16.0 & 2.0 & 2.1 & 5.4 & 1.6 & 5.3 & 1.5 \\
\hline 177 & 27608 & 9 & 1 & 40.0 & 10.0 & -2.0 & 2.0 & 2.0 & 1.1 & 5.8 & 1.2 \\
\hline 177 & 27608 & 9 & 2 & 73.0 & 14.0 & 3.6 & 1.0 & 2.9 & 1.3 & 6.1 & 1.3 \\
\hline 179 & 27944 & 1 & 2 & 48.0 & 12.0 & -1.7 & 2.1 & 3.3 & 1.7 & 7.6 & 1.3 \\
\hline 179 & 27944 & 2 & 1 & 38.0 & 12.0 & 3.4 & 1.8 & 5.0 & 1.2 & 5.2 & 1.1 \\
\hline 179 & 27941 & 3 & 1 & 35.0 & 10.0 & 3.2 & .5 & 8.1 & 2.0 & .8 & $1.2 *$ \\
\hline 181 & 28242 & 1 & 1 & 60.2 & 14.8 & -3.2 & 2.3 & 2.9 & 1.3 & .6 & .4* \\
\hline 181 & 20242 & 2 & 1 & 47.0 & 11.0 & & & 3.7 & 1.2 & .0 & 1.00 \\
\hline
\end{tabular}


TABLE 7. (contd)

\begin{tabular}{|c|c|c|c|c|c|c|c|c|c|c|c|}
\hline \multirow{3}{*}{$\begin{array}{l}\text { STAKE } \\
\text { MO. } \\
181\end{array}$} & \multirow{2}{*}{$\begin{array}{l}\text { DISTAMCE } \\
\text { METERS }\end{array}$} & \multicolumn{2}{|c|}{ SAMPLE } & \multicolumn{2}{|c|}{ THORIUH } & \multicolumn{2}{|c|}{ RADIUn } & \multicolumn{2}{|c|}{ LEAD } & \multicolumn{2}{|c|}{ URANIUN } \\
\hline & & TYPE & REP. & LEVEL & ERROR & LEUEL & ERROR & LEVEL & ERROR & LEVEL & ERROR \\
\hline & 28242 & 2 & 2 & 90.0 & 17.0 & .1 & 2.1 & 4.2 & 1.6 & 6.2 & 1.5 \\
\hline 181 & 28242 & 3 & 1 & 165.0 & 23.0 & 1.4 & 3.1 & 1.8 & 2.6 & 14.0 & 2.0 \\
\hline 181 & 28242 & 7 & 1 & 30.0 & 12.0 & -4.8 & 1.8 & 2.7 & 1.4 & 6.0 & 1.2 \\
\hline 183 & 28525 & 1 & 1 & 72.6 & 16.7 & -2.6 & 2.2 & 3.0 & 1.5 & 8.1 & 1.6 \\
\hline 183 & 28525 & 1 & 2 & 37.0 & 14.0 & -10.9 & 2.7 & 3.5 & 1.6 & 1.1 & .5 \\
\hline 183 & 28525 & 2 & 1 & 40.0 & 11.0 & 3.2 & 1.9 & 1.7 & 1.0 & 3.9 & 1.1 \\
\hline 193 & 28525 & 2 & 2 & 59.4 & 12.1 & .1 & 2.0 & 1.1 & 2.0 & 5.8 & 1.2 \\
\hline 183 & 28525 & 5 & 1 & 39.0 & 13.0 & & & 2.9 & 1.7 & .0 & $1.1 \%$ \\
\hline 185 & 28772 & 1 & 1 & 35.0 & 12.0 & 1.6 & 2.0 & 3.9 & 1.2 & -.6 & $1.1 *$ \\
\hline 185 & 28772 & 1 & 2 & 39.0 & 15.0 & 3.7 & 1.8 & 4.6 & 1.6 & -.5 & $1.0 *$ \\
\hline 185 & 28772 & 2 & 1 & 35.0 & 11.0 & 5.7 & 1.7 & 1.7 & .9 & .5 & $1.1 *$ \\
\hline 185 & 28772 & 2 & 2 & 58.0 & 14.0 & & & 1.5 & 1.7 & -.1 & . B* \\
\hline 185 & 28772 & 4 & 1 & 51.0 & 16.0 & & & 4.4 & 1.9 & .6 & $1.1 *$ \\
\hline 187 & 29101 & 1 & 1 & 49.6 & 15.0 & 1.8 & 1.9 & 2.1 & 1.5 & 2.8 & 1.3 \\
\hline 187 & 29101 & 3 & 1 & 54.0 & 15.0 & & & 4.0 & 1.5 & 1.3 & $1.0 *$ \\
\hline 188 & 29271 & 5 & 1 & 30.0 & 13.0 & & & 2.3 & 1.5 & 1.0 & $.9 *$ \\
\hline 189 & 29514 & 1 & 1 & 32.0 & 13.0 & & & .4 & 1.6 & .8 & $1.0 *$ \\
\hline 189 & 29514 & 5 & 1 & 32.0 & 14.0 & & & 3.1 & 1.5 & -.7 & $1.2 *$ \\
\hline 191 & 29833 & 4 & 1 & 104.0 & 18.0 & 11.0 & 1.2 & 2.9 & 1.8 & .0 & $1.0 *$ \\
\hline 191 & 29833 & 4 & 2 & 71.0 & 18.0 & 1.2 & .2 & .2 & 1.6 & -.3 & $1.1 *$ \\
\hline 193 & 30123 & 1 & 2 & 45.0 & 12.0 & 1.0 & .1 & 3.2 & 1.1 & .5 & $.9 *$ \\
\hline 193 & 30123 & 2 & 2 & 73.0 & 15.0 & -1.8 & 2.1 & 5.4 & 1.7 & 7.4 & 1.5 \\
\hline 193 & 30123 & 4 & 1 & 57.0 & 12.0 & -.9 & 2.0 & 1.1 & 1.1 & 5.0 & 1.2 \\
\hline 195 & 30320 & 1 & 1 & 30.0 & 10.0 & & & 1.6 & 1.0 & -.1 & $1.1 \%$ \\
\hline 195 & 30320 & 1 & 2 & 44.0 & 17.0 & & & 3.8 & 1.8 & -.7 & $1.0 *$ \\
\hline 195 & 30320 & 3 & 1 & 115.0 & 23.0 & 8.1 & 2.5 & 13.0 & 2.6 & 2.0 & $1.5 *$ \\
\hline 195 & 30320 & 5 & 1 & 30.0 & 9.1 & & & .8 & .8 & -.6 & $.9=$ \\
\hline 197 & 30644 & 1 & 2 & 122.2 & 19.0 & -.3 & 2.3 & 3.6 & 1.6 & 9.8 & 1.7 \\
\hline 197 & 30644 & 2 & 1 & 37.0 & 13.0 & & & 2.3 & 1.7 & -.1 & $.8 *$ \\
\hline 198 & 30819 & 4 & 1 & 43.0 & 15.0 & & & 3.4 & 1.8 & -.7 & $.9 *$ \\
\hline 198 & 30819 & 5 & 1 & 81.0 & 19.0 & 1.8 & .5 & 2.3 & 2.0 & -1.3 & $1.1 *$ \\
\hline 199 & 30970 & 1 & 1 & 34.0 & 12.0 & .1 & 2.0 & 3.3 & 1.3 & 5.7 & 1.3 \\
\hline 199 & 30970 & 2 & 1 & 40.7 & 12.4 & -1.1 & 1.9 & .4 & 1.4 & .3 & $.5 *$ \\
\hline 199 & 30970 & 2 & 2 & 89.0 & 19.0 & 1.2 & .1 & 4.4 & 2.0 & .0 & $1.1 *$ \\
\hline 199 & 30970 & 3 & 1 & 67.0 & 14.0 & & & 6.2 & 1.4 & -.3 & $1.3 *$ \\
\hline 201 & 31239 & 4 & 1 & 48.0 & 12.0 & 2.6 & .5 & 4.1 & 1.3 & 2.0 & $.9 *$ \\
\hline 201 & 31239 & $b$ & 2 & 34.0 & 14.0 & 1.4 & .2 & 2.3 & 1.4 & 1.0 & $1.0 *$ \\
\hline 201 & 31239 & 7 & 2 & 154.1 & 21.7 & -4.1 & 2.4 & 6.7 & 1.9 & 13.0 & 1.9 \\
\hline 203 & 31479 & 2 & 2 & 35.0 & 14.0 & 1.3 & .3 & 2.3 & 1.5 & 1.4 & $1.1 *$ \\
\hline 203 & 31479 & 3 & 1 & 50.0 & 16.0 & 7.2 & 1.9 & 3.8 & 1.9 & 1.6 & $1.2 *$ \\
\hline 205 & 31745 & 1 & 1 & 31.0 & 11.0 & 3.3 & 1.6 & 2.1 & 1.0 & .2 & $.9 *$ \\
\hline
\end{tabular}


TABLE 7. (contd)

\begin{tabular}{|c|c|c|c|c|c|c|c|c|c|c|c|}
\hline STAKE & DISTANCE & SAH & & Tho & & & & & & UR & \\
\hline no. & METERS & TYPE & REP. & LEVEL & ERROR & LEVEL & ERROR & LEVEL & ERROR & LEVEL & ERROR \\
\hline 205 & 31745 & 2 & 1 & 32.0 & 12.0 & 4.6 & 1.5 & 1.9 & 1.4 & -.1 & $1.0 *$ \\
\hline 205 & 31745 & 4 & 1 & 30.0 & 10.0 & 1.6 & 1.0 & .4 &.$\theta$ & -.4 & $.9 *$ \\
\hline 207 & 32052 & 2 & 1 & 32.2 & 10.9 & -2.2 & 2.2 & .9 & 2.0 & 5.7 & 1.2 \\
\hline 207 & 32052 & 2 & 2 & 33.7 & 15.3 & -.3 & 1.7 & .2 & 1.4 & 4.4 & 1.4 \\
\hline 207 & 32052 & 3 & 1 & 37.1 & 10.6 & -.3 & 2.0 & 2.1 & 1.1 & 3.0 & 1.1 \\
\hline 213 & 33053 & 2 & 1 & 51.0 & 13.0 & 4.2 & 1.7 & 1.9 & 1.4 & .1 & $3.6 *$ \\
\hline 215 & 33353 & 2 & 1 & 32.0 & 17.0 & & & -.7 & 1.8 & -.9 & $1.0 *$ \\
\hline 217 & 33684 & 1 & 1 & 11.0 & 12.0 & & & 1.5 & 1.1 & .4 & $1.0 *$ \\
\hline 217 & 33684 & 1 & 2 & 55.0 & 14.0 & .8 & .1 & 5.0 & 1.6 & .2 & $.9 *$ \\
\hline 219 & 33960 & 3 & 1 & 40.0 & 11.0 & .8 & .1 & 2.6 & 1.0 & .2 & $1.0 *$ \\
\hline 221 & 34327 & 1 & 2 & 37.1 & 8.6 & -1.8 & 1.7 & 2.5 & .9 & 4.5 & 1.0 \\
\hline 223 & 34639 & 2 & 1 & 30.0 & 13.0 & & & 1.7 & 1.5 & -1.1 & $.8 *$ \\
\hline 225 & 34939 & 3 & 1 & 77.0 & 15.0 & & & 2.2 & 1.2 & 1.3 & $1.0 *$ \\
\hline 225 & 34939 & 3 & 2 & 41.0 & 12.0 & 5.5 & 1.7 & 1.1 & 1.1 & .9 & $1.0 *$ \\
\hline 225 & 34939 & 4 & 1 & 103.0 & 19.0 & & & 5.6 & 1.9 & .8 & $1.2 *$ \\
\hline 227 & 35215 & 1 & 1 & 64.0 & 13.0 & -7.5 & 2.6 & 3.4 & 1.1 & .8 & .5 \\
\hline 227 & 35215 & 1 & 2 & 44.0 & 14.0 & .8 & .1 & 1.5 & 1.4 & .8 & $1.0 *$ \\
\hline 229 & 35477 & 1 & 1 & 44.3 & 14.1 & -5.4 & 2.1 & 5.2 & 1.6 & .7 & .40 \\
\hline 231 & 35784 & 1 & 1 & 35.0 & 10.0 & .8 & .1 & 1.2 & 1.0 & .5 & $.7 *$ \\
\hline 231 & 35784 & 1 & 2 & 101.5 & 14.8 & -15.4 & 3.2 & 6.2 & 1.3 & 1.5 & $.3 *$ \\
\hline 231 & 35784 & 8 & 2 & 43.0 & 13.0 & & & -.1 & 1.5 & 1.2 & $.9 *$ \\
\hline 233 & 36118 & 1 & 1 & 143.0 & 22.0 & & & 4.6 & 2.0 & .8 & $1.4 *$ \\
\hline 233 & 36118 & 2 & 1 & 11.0 & 16.0 & 1.3 & .2 & 3.1 & 1.7 & .8 & $1.0 *$ \\
\hline 235 & 36435 & 2 & 1 & 35.0 & 11.0 & & & 3.6 & 1.1 & .2 & $1.0 *$ \\
\hline 237 & 36850 & 4 & 1 & 35.0 & 16.0 & & & 4.4 & 1.8 & -1.1 & $1.2 *$ \\
\hline 239 & 37191 & 1 & 2 & 90.0 & 21.0 & 2.9 & .5 & 4.8 & 2.0 & -.7 & $1.3 *$ \\
\hline 239 & 37191 & 2 & 1 & 41.0 & 14.0 & 3.0 & 1.8 & 1.0 & 1.4 & .1 & .4 \\
\hline 239 & 37191 & 2 & 2 & 110.0 & 19.0 & 1.5 & .2 & 4.2 & 1.8 & -.1 & $1.3 *$ \\
\hline 241 & 37477 & 2 & 2 & 31.8 & 13.5 & 1.0 & 2.1 & 2.5 & 1.5 & 5.7 & 1.4 \\
\hline 243 & 37813 & 2 & 2 & 39.0 & 12.0 & -2.2 & 2.1 & .4 & 1.5 & .5 & .4 \\
\hline 245 & 38096 & 2 & 1 & 16.0 & 13.0 & 1.7 & 1.8 & -1.4 & 1.5 & 1.4 & $1.0 *$ \\
\hline 247 & 38410 & 1 & 1 & 30.0 & 12.0 & .9 & .1 & .2 & 1.5 & .0 & .90 \\
\hline 249 & 38715 & 1 & 1 & 32.1 & 10.6 & -3.0 & 2.2 & 3.2 & .9 & .4 & $.4 \%$ \\
\hline 249 & $38>15$ & 2 & 1 & 60.2 & 14.2 & 1.3 & 1.9 & 2.1 & 1.3 & 4.8 & 1.3 \\
\hline 253 & 39291 & 1 & 2 & 40.7 & 13.3 & -.4 & 1.9 & 6.5 & 1.6 & 6.0 & 1.4 \\
\hline 257 & 39920 & 2 & 1 & 40.7 & 13.8 & -.2 & 1.7 & 1.7 & 1.3 & 4.8 & 1.3 \\
\hline 259 & 40225 & 1 & 1 & 30.1 & 15.1 & 1.5 & 1.6 & 1.9 & 1.4 & 2.0 & 1.3 \\
\hline 259 & 40225 & 2 & 1 & 49.5 & 12.1 & -.8 & 2.2 & 1.4 & 1.0 & 7.7 & 1.2 \\
\hline 261 & 40551 & 1 & 1 & 47.8 & 13.8 & -6.3 & 2.3 & 1.7 & 1.3 & .7 & $.4 *$ \\
\hline 261 & 40551 & 2 & 1 & 61.9 & 12.3 & -.0 & 1.7 & 2.6 & 1.2 & 4.6 & 1.1 \\
\hline 261 & 40551 & 7 & 1 & 44.3 & 13.1 & -1.3 & 1.6 & 2.5 & 1.2 & 3.7 & 1.1 \\
\hline
\end{tabular}


TABLE 7. (contd)

\begin{tabular}{|c|c|c|c|c|c|c|c|c|c|c|c|}
\hline \multirow{3}{*}{$\begin{array}{l}\text { STAKE } \\
\text { MO. } \\
2 b 1\end{array}$} & \multirow{2}{*}{$\begin{array}{l}\text { DISTAMCE } \\
\text { WETERS }\end{array}$} & \multicolumn{2}{|c|}{ SAMPLE } & \multicolumn{2}{|c|}{ THORIUИ } & \multicolumn{2}{|c|}{ RADIUY } & \multicolumn{2}{|c|}{ LEAD } & \multicolumn{2}{|c|}{ URAMIUN } \\
\hline & & TYPE & REP. & LEVEL & ERROR & LEVEL & ERROR & LEUEL & ERROR & LEVEL & ERROR \\
\hline & 40551 & 8 & 1 & 30.9 & 8.5 & 1.4 & 1.8 & .1 & .9 & 3.7 & .9 \\
\hline 263 & 40897 & 1 & 2 & 30.1 & 12.9 & -9.8 & 2.6 & 5.2 & 1.5 & 1.0 & $.5 *$ \\
\hline 263 & 10897 & 2 & 1 & 41.0 & 14.0 & & & 1.5 & 1.5 & .8 & $.9 *$ \\
\hline 265 & 41171 & 1 & 2 & 30.0 & 14.0 & .6 & 1.5 & 2.9 & 1.7 & .8 & $1.2 *$ \\
\hline 267 & 41404 & 1 & 2 & 50.0 & 11.0 & .7 & .2 & 3.6 & 1.1 & 1.8 & $.9 *$ \\
\hline 271 & 42184 & 1 & 2 & 44.0 & 14.0 & .9 & .1 & 1.0 & 1.5 & .2 & $1.2 *$ \\
\hline 271 & 42184 & 2 & 2 & 47.0 & 11.3 & 1.6 & 1.9 & 1.1 & .9 & 2.8 & 1.0 \\
\hline 271 & 42184 & 7 & 2 & 38.4 & 10.3 & -14.5 & 2.6 & .1 & .9 & 1.1 & $.4 *$ \\
\hline 271 & 42184 & 7 & 3 & 35.4 & 13.5 & 9.1 & 2.0 & 2.1 & 1.5 & .6 & $.5 *$ \\
\hline 273 & 42554 & 1 & 3 & 47.8 & 15.9 & 3.1 & 1.9 & 1.7 & 1.4 & 3.9 & 1.4 \\
\hline 275 & 42871 & 2 & 1 & 41.0 & 11.0 & .3 & 1.9 & 2.8 & 1.0 & 4.1 & 1.1 \\
\hline 277 & 43195 & 1 & 1 & 35.9 & 11.4 & -1.3 & 2.1 & 3.7 & 1.1 & 6.3 & 1.1 \\
\hline 277 & 43193 & 1 & 2 & 60.2 & 13.9 & -.4 & 1.9 & .0 & 1.6 & 5.7 & 1.4 \\
\hline 279 & 43382 & 1 & 1 & 38.4 & 9.7 & -1.9 & 2.0 & 4.5 & 1.0 & 5.3 & 1.1 \\
\hline 279 & 43382 & 2 & 1 & 31.9 & 11.8 & -2.1 & 1.7 & 1.4 & 1.2 & 3.6 & 1.3 \\
\hline 281 & 43723 & 1 & 1 & 31.8 & 14.6 & 1.6 & 2.0 & 1.3 & 1.4 & 5.7 & 1.4 \\
\hline 281 & 43723 & 2 & 1 & 70.8 & 16.9 & .4 & 2.2 & 4.3 & 1.4 & 7.4 & 1.8 \\
\hline 281 & 43723 & 7 & 1 & 42.0 & 14.0 & .4 & 1.9 & 4.0 & 1.5 & 4.7 & 1.4 \\
\hline 281 & 43723 & 8 & 1 & 30.4 & 11.4 & 2.2 & 1.8 & 3.9 & 1.1 & 2.4 & 1.2 \\
\hline 283 & 14138 & 1 & 1 & 34.0 & 15.0 & -2.8 & 2.0 & 1.0 & 1.3 & 6.7 & 1.6 \\
\hline 283 & 44138 & 2 & 1 & 70.8 & 14.6 & -.5 & 2.2 & 3.3 & 1.4 & 7.2 & 1.4 \\
\hline 285 & 44371 & 1 & 1 & 51.0 & 15.0 & 3.7 & 1.8 & 3.6 & 1.6 & .0 & .90 \\
\hline 287 & 44745 & 1 & 1 & 57.0 & 16.0 & -.9 & 2.1 & 3.8 & 1.5 & 7.4 & 1.5 \\
\hline 287 & 44745 & 1 & 2 & 48.0 & 16.0 & -.9 & 2.3 & 2.1 & 1.6 & 11.0 & 1.7 \\
\hline 287 & 44745 & 2 & 1 & 11.0 & 12.0 & -.7 & 1.9 & 2.2 & 1.1 & 3.8 & 1.2 \\
\hline 289 & 45032 & 1 & 1 & 63.7 & 17.5 & -1.8 & 2.3 & 4.2 & 1.8 & 10.7 & 1.7 \\
\hline 289 & 45052 & 2 & 1 & 129.0 & 16.0 & -4.3 & 2.6 & 5.4 & 1.4 & 11.0 & 1.5 \\
\hline 291 & 45376 & 1 & 1 & 52.0 & 12.6 & 1.8 & 2.4 & 3.0 & 1.3 & 7.4 & 1.3 \\
\hline 291 & 45376 & 1 & 2 & 63.1 & 14.1 & 3.5 & 2.3 & 4.4 & 1.2 & 8.0 & 1.3 \\
\hline 291 & 45376 & 2 & 1 & 109.8 & 19.7 & -1.4 & 2.5 & 4.4 & 2.0 & 11.8 & 1.9 \\
\hline 291 & 45376 & 7 & 1 & 38.0 & 16.0 & 2.7 & 2.2 & 3.8 & 1.6 & 7.4 & 1.6 \\
\hline 291 & 45376 & B & 1 & 96.0 & 15.0 & .4 & 2.3 & 3.1 & 1.3 & 8.7 & 1.4 \\
\hline 291 & 45376 & 8 & 3 & 49.0 & 7.1 & 2.9 & 2.1 & 2.7 & 1.0 & 4.6 & 1.0 \\
\hline 292 & 45548 & 1 & 1 & 35.0 & 13.0 & 4.0 & 1.5 & 3.8 & 1.4 & .0 & $.9 *$ \\
\hline 295 & 45997 & 1 & 1 & 85.0 & 12.0 & 6.8 & 2.0 & 4.2 & 1.1 & -.1 & $1.0 *$ \\
\hline 297 & 46439 & 2 & 2 & 46.1 & 14.6 & -1.1 & 1.6 & 1.0 & 1.1 & 4.3 & 1.3 \\
\hline 303 & 47454 & 2 & 1 & 45.8 & 11.9 & -2.1 & 2.4 & 3.4 & 1.1 & 9.0 & 1.3 \\
\hline 305 & 47795 & 1 & 2 & 115.1 & 16.9 & -3.1 & 2.0 & 2.9 & 1.5 & 7.2 & 1.5 \\
\hline 305 & 47795 & 3 & 1 & 50.0 & 11.0 & 4.9 & 1.9 & 3.0 & 1.2 & .2 & $.9 *$ \\
\hline 307 & $y$ & 2 & 1 & 33.1 & 13.3 & 6.0 & 1.9 & 2.7 & 1.3 & 1.3 & 1.3 \\
\hline 309 & & 1 & 2 & 30.1 & 12.4 & 1.4 & 1.0 & .8 & 1.3 & 3.8 & 1.3 \\
\hline
\end{tabular}


TABLE 7. (contd)

\begin{tabular}{|c|c|c|c|c|c|c|c|c|c|c|c|}
\hline \multirow{3}{*}{$\begin{array}{l}\text { STAKE } \\
\text { MO. } \\
309\end{array}$} & \multirow{3}{*}{$\begin{array}{l}\text { DISTAMCE } \\
\text { METERS }\end{array}$} & \multicolumn{2}{|c|}{ SAMPLE } & \multicolumn{2}{|c|}{ THORIUK } & \multicolumn{2}{|c|}{ RAEIUH } & \multicolumn{2}{|c|}{ LEAD } & \multicolumn{2}{|c|}{ URAMIUN } \\
\hline & & TYPE & REP. & LEVEL & ERROR & LEVEL & ERROR & LEVEL & ERROR & LEVEL & ERROR \\
\hline & & 2 & 2 & 42.5 & 14.6 & -.8 & 2.1 & .4 & 1.4 & 3.5 & 1.5 \\
\hline 309 & & 5 & 1 & 42.5 & 13.7 & .6 & 2.0 & -.3 & 1.2 & 5.5 & 1.4 \\
\hline 311 & & 2 & i & 53.2 & 11.4 & .2 & 2.2 & 3.2 & 1.0 & 6.7 & 1.2 \\
\hline 311 & & 2 & 2 & 74.4 & 14.6 & -2.0 & 1.8 & 3.8 & 1.6 & 4.7 & 1.3 \\
\hline 311 & & 7 & 1 & 60.3 & 14.2 & 1.1 & 2.0 & .8 & 1.4 & 5.1 & 1.3 \\
\hline 311 & & 7 & 2 & 38.4 & 11.1 & 1.2 & 2.2 & 5.0 & 1.1 & 6.1 & 1.2 \\
\hline 311 & & 8 & 1 & 30.1 & 12.1 & 1.9 & 1.7 & 4.0 & 1.3 & 2.3 & 1.2 \\
\hline 319 & & 1 & 1 & 42.5 & 17.2 & -2.1 & 2.4 & 5.2 & 1.8 & 8.3 & 1.6 \\
\hline 321 & & 1 & 2 & 40.7 & 11.3 & -.2 & 1.9 & 1.9 & 1.5 & 4.5 & 1.3 \\
\hline 321 & & 4 & 1 & 76.0 & 16.0 & 4.0 & 1.7 & 5.6 & 2.0 & -1.1 & $1.0 *$ \\
\hline 323 & & 1 & 1 & 35.4 & 13.2 & -1.4 & 1.3 & .0 & 1.4 & 3.0 & 1.2 \\
\hline 323 & & $i$ & 2 & 53.1 & 14.2 & .2 & 2.1 & 4.6 & 1.4 & 7.7 & 1.4 \\
\hline 323 & & 9 & 1 & $7 b .2$ & 16.7 & -.1 & 2.1 & 4.8 & 1.7 & 8.3 & 1.6 \\
\hline 325 & & 1 & 1 & 33.0 & 11.0 & & & 1.9 & 1.1 & 1.1 & $1.0 *$ \\
\hline 327 & & 1 & 1 & 31.0 & 9.3 & -3.2 & 1.6 & 1.0 & .9 & 4.5 & 1.0 \\
\hline 327 & & 1 & 2 & 60.3 & 14.6 & 2.8 & 1.8 & 2.1 & 1.3 & 3.4 & 1.4 \\
\hline 327 & & 2 & $i$ & 92.1 & 17.4 & -2.0 & 2.3 & 2.3 & 1.8 & 11.6 & 1.7 \\
\hline 329 & & 1 & 2 & 37.2 & 11.1 & .5 & 1.9 & 2.3 & -1.0 & 4.8 & 1.1 \\
\hline 331 & & 4 & 2 & 64.0 & 16.0 & 3.7 & 1.8 & 3.3 & 1.8 & 2.3 & $1.2 *$ \\
\hline 333 & & 1 & 1 & 37.2 & 14.7 & -.6 & 1.8 & 2.9 & 1.4 & 4.8 & 1.3 \\
\hline 333 & & 4 & 1 & 63.7 & 17.2 & -1.2 & 2.5 & 5.8 & 2.0 & 7.9 & 1.7 \\
\hline 335 & & 4 & $i$ & 134.9 & 17.7 & -2.1 & 2.9 & 13.9 & 1.8 & 14.8 & 1.8 \\
\hline 337 & & 1 & 1 & 111.6 & 16.3 & 1.4 & 2.3 & 5.6 & 1.5 & 8.5 & 1.5 \\
\hline 337 & & 1 & 2 & 31.9 & 14.6 & -1.8 & 1.8 & 2.9 & 1.5 & 5.3 & 1.4 \\
\hline 337 & & 2 & 1 & 42.3 & 12.3 & .3 & 2.2 & 4.0 & 1.5 & 5.0 & 1.2 \\
\hline 339 & & 1 & 2 & 48.3 & 13.5 & 1.8 & 2.3 & 7.7 & 1.5 & 6.4 & 1.4 \\
\hline 339 & & 1 & 3 & 10.7 & 14.3 & -3.3 & 2.1 & 1.0 & 1.3 & 5.8 & 1.5 \\
\hline 339 & & 2 & 1 & 125.8 & 18.5 & -2.1 & 2.4 & 6.9 & 1.8 & 10.9 & 1.7 \\
\hline 341 & & 1 & 2 & 34.7 & 10.1 & -.9 & 1.8 & 2.5 & 1.0 & 4.7 & 1.1 \\
\hline 341 & & 4 & 2 & 102.7 & 20.8 & 3.3 & 3.0 & 14.8 & 2.6 & 13.7 & 2.0 \\
\hline 341 & & 4 & 3 & 40.7 & 13.6 & -3.8 & 2.3 & 5.4 & 1.6 & 12.4 & 1.6 \\
\hline 341 & & 3 & 1 & 12.5 & 15.6 & .5 & 2.2 & 2.7 & 1.6 & 7.2 & 1.6 \\
\hline 341 & & 8 & 1 & 31.9 & 13.0 & 3.0 & 2.3 & 4.4 & 1.5 & 4.8 & 1.3 \\
\hline 343 & & 3 & 1 & 125.7 & 20.6 & -.9 & 3.1 & 8.3 & 2.2 & 14.1 & 2.0 \\
\hline 343 & & 3 & 2 & 115.1 & 17.5 & 1.7 & 2.6 & 13.2 & 1.8 & 11.6 & 1.7 \\
\hline 345 & & 3 & 1 & 92.1 & 19.1 & -.5 & 2.1 & 6.1 & 1.7 & 7.6 & 1.7 \\
\hline 347 & & 1 & 2 & 16.0 & 11.7 & -1.0 & 2.0 & -.9 & 1.3 & 5.0 & 1.2 \\
\hline 347 & & 3 & 2 & 126.3 & 17.5 & 2.4 & 3.2 & 13.5 & 1.6 & 14.2 & 1.7 \\
\hline 349 & & 1 & i & 69.1 & 16.9 & -1.6 & 2.4 & 3.3 & 1.6 & 9.6 & 1.6 \\
\hline 349 & & 3 & 1 & 07.9 & 15.7 & -3.3 & 2.3 & 6.3 & 1.5 & 10.5 & 1.5 \\
\hline 351 & & 1 & 2 & 70.9 & 16.4 & -3.7 & 2.1 & 3.0 & 1.0 & 6.5 & 1.5 \\
\hline
\end{tabular}


TABLE 7. (contd)

\begin{tabular}{|c|c|c|c|c|c|c|c|c|c|c|c|}
\hline \multirow{3}{*}{$\begin{array}{l}\text { STAKE } \\
\text { WO. } \\
351\end{array}$} & \multirow{2}{*}{$\begin{array}{l}\text { DISTAMCE } \\
\text { METERS }\end{array}$} & \multicolumn{2}{|c|}{ SAMPLE } & \multicolumn{2}{|c|}{ THORIUA } & \multicolumn{2}{|c|}{ RABIUM } & \multicolumn{2}{|c|}{ LEAD } & \multicolumn{2}{|c|}{ URAMIUM } \\
\hline & & TYPE & REP. & LEVEL & ERROR & LEVEL & ERROR & LEUEL & ERROR & LEVEL & ERROR \\
\hline & & 3 & 1 & 111.4 & 17.2 & -1.9 & 2.9 & 12.0 & 1.9 & 12.4 & 1.8 \\
\hline 351 & & 8 & 1 & 53.1 & 13.7 & .7 & 2.1 & 2.7 & 1.7 & 7.3 & 1.5 \\
\hline 353 & & 3 & 1 & 131.1 & 22.4 & 5.9 & 2.9 & 9.8 & 2.5 & 5.7 & 2.0 \\
\hline 335 & & 1 & 1 & 32.0 & 13.0 & & & 3.1 & 1.6 & .2 & $1.0 *$ \\
\hline 357 & & 2 & 2 & 47.8 & 16.5 & 4.8 & 2.3 & 4.1 & 1.7 & 4.3 & 1.5 \\
\hline 359 & & 2 & 2 & 39.6 & 10.7 & .8 & 2.1 & 2.9 & 1.1 & 4.9 & 1.1 \\
\hline 359 & & 3 & 1 & 107.7 & 19.0 & -20.3 & 3.7 & 14.1 & 1.9 & 2.0 & $.6 *$ \\
\hline 363 & & 2 & 1 & 31.9 & 13.3 & .8 & 1.7 & 1.3 & 1.4 & 3.1 & 1.4 \\
\hline 363 & & 3 & 1 & 39.6 & 13.2 & 20.2 & .1 & 2,3 & 1.1 & -.7 & $.5 *$ \\
\hline 365 & & 2 & 1 & 51.0 & 11.0 & 2.2 & 1.6 & 4.3 & 1.2 & -.1 & $1.1 *$ \\
\hline 365 & & 3 & 1 & 44.5 & 11.2 & 1.2 & 2.1 & 2.3 & 1.1 & .2 & . 4* \\
\hline 367 & & 3 & 1 & 118.7 & 20.1 & . B & 3.1 & 13.8 & 2.2 & .9 & $.8 *$ \\
\hline 369 & & 3 & 1 & 33.0 & 10.0 & & & .4 & 1.0 & -.4 & .9 \\
\hline 371 & & 2 & 1 & 37.1 & 10.7 & -.5 & 1.6 & 4.3 & 1.0 & 3.4 & 1.1 \\
\hline 373 & & 1 & 1 & 35.9 & 10.4 & 2.9 & 2.1 & 2.5 & .9 & 4.9 & 1.1 \\
\hline 373 & & 1 & 2 & 10.7 & 13.4 & -.6 & 2.0 & 1.9 & 1.4 & 6.1 & 1.4 \\
\hline 373 & & 3 & 1 & 45.8 & 12.2 & .4 & 2.1 & 5.0 & 1.1 & 7.1 & 1.2 \\
\hline 373 & & 4 & 1 & 141.7 & 22.9 & -.5 & 2.8 & 14.0 & 2.5 & 13.0 & 2.0 \\
\hline 373 & & 5 & 1 & 62.0 & 17.6 & 2.5 & 2.5 & 8.4 & 1.9 & 6.5 & 1.6 \\
\hline 375 & & 3 & 1 & 33.3 & 10.1 & .2 & 1.9 & 5.1 & 1.1 & 4.9 & 1.1 \\
\hline 377 & & 1 & 2 & 46.1 & 14.2 & -1.6 & 2.4 & 7.7 & 1.8 & 7.5 & 1.6 \\
\hline 377 & & 3 & 1 & 47.0 & 12.1 & .8 & 2.3 & 3.2 & 1.2 & 9.0 & 1.3 \\
\hline 379 & & 1 & 1 & 31.9 & 14.0 & .6 & 2.1 & 2.9 & 1.6 & 3.3 & 1.5 \\
\hline 381 & & 1 & 2 & 31.9 & 13.5 & -.9 & 1.9 & 2.3 & 1.6 & 4.8 & 1.4 \\
\hline 383 & & 1 & 1 & 53.2 & 12.2 & .3 & 2.3 & 2.8 & 1.1 & 7.2 & 1.2 \\
\hline 385 & & 3 & 1 & 74.3 & 14.9 & -.7 & 2.6 & 7.9 & 1.5 & 12.4 & 1.6 \\
\hline 387 & & 1 & 1 & 54.9 & 14.5 & -.8 & 2.1 & 1.5 & 1.6 & 7.6 & 1.5 \\
\hline 387 & & 3 & 1 & 99.2 & 18.1 & -1.1 & 2.5 & 6.7 & 1.8 & 9.3 & 1.7 \\
\hline 387 & & 5 & 1 & 47.0 & 11.1 & 2.8 & 2.1 & 4.4 & 1.0 & 4.8 & 1.2 \\
\hline 389 & & 3 & 1 & 93.9 & 19.3 & -.0 & 2.6 & 5.2 & 2.0 & 9.4 & 1.7 \\
\hline 389 & & 9 & 1 & 47.0 & 11.4 & 2.7 & 3.8 & 2.6 & 1.0 & 27.6 & 1.9 \\
\hline 391 & & 3 & 1 & 138.7 & 22.7 & 6.4 & 2.9 & 16.1 & 2.4 & 9.7 & 2.0 \\
\hline 391 & & 7 & 1 & 47.0 & 11.3 & 3.4 & 2.1 & 1.4 & 1.0 & 6.1 & 1.2 \\
\hline 393 & & 4 & 1 & 73.0 & 13.0 & 12.8 & 1.5 & 5.8 & 1.2 & -.2 & $.3 *$ \\
\hline 395 & & 1 & 2 & 30.0 & 10.0 & & & 1.1 & 1.1 & 2.7 & $1.2 *$ \\
\hline 395 & & 4 & 1 & 39.0 & 12.0 & & & 3.3 & 1.7 & 2.0 & $1.1 *$ \\
\hline 395 & & 4 & 3 & 47.0 & 12.0 & & . & 5.8 & 1.3 & .5 & $.9 *$ \\
\hline 397 & & 4 & 1 & 30.1 & 14.7 & 7.9 & 2.1 & 4.4 & 1.5 & -.8 & $.5 *$ \\
\hline 401 & & 1 & 1 & 40.9 & 11.7 & -2.8 & 1.9 & 2.2 & 1.0 & 6.5 & 1.3 \\
\hline 401 & & 8 & 1 & 35.0 & 2.4 & 4.6 & .4 & 2.4 & .2 & .1 & $.2 *$ \\
\hline 405 & & 1 & 1 & 57.0 & 13.0 & & & 1.9 & 1.7 & 1.0 & $.9 *$ \\
\hline
\end{tabular}


TABLE 7. (contd)

\begin{tabular}{|c|c|c|c|c|c|c|c|c|c|c|c|}
\hline \multirow{3}{*}{$\begin{array}{l}\text { STAKE } \\
\text { NO. } \\
405\end{array}$} & \multirow{2}{*}{$\begin{array}{l}\text { DISTAMCE } \\
\text { METERS }\end{array}$} & \multicolumn{2}{|c|}{ SAMPLE } & \multicolumn{2}{|c|}{ THORIUN } & \multicolumn{2}{|c|}{ RADIUY } & \multicolumn{2}{|c|}{ LEAD } & \multicolumn{2}{|c|}{ URAWIUH } \\
\hline & & TYPE & REP. & LEVEL & ERRDR & LEVEL & ERROR & LEVEL & ERROR & LEVEL & ERROR \\
\hline & & 4 & 1 & 31.0 & 9.2 & & & 1.2 & 1.0 & .4 & $9.2 *$ \\
\hline 405 & & 4 & 2 & 53.0 & 17.0 & & & 3.8 & 1.7 & 1.0 & $1.1 *$ \\
\hline 409 & & 1 & 2 & 129.3 & 17.8 & 1.5 & 2.6 & 9.6 & 2.1 & 10.0 & 1.7 \\
\hline 411 & & 1 & 2 & 79.7 & 18.7 & .1 & 2.5 & 3.8 & 1.7 & 10.8 & 1.8 \\
\hline 411 & & 2 & 2 & 39.0 & 15.4 & -1.0 & 2.0 & 3.3 & 1.4 & 5.3 & 1.5 \\
\hline 413 & & 1 & 2 & 14.3 & 16.7 & 2.4 & 2.2 & 2.7 & 1.6 & 5.7 & 1.6 \\
\hline 413 & & 1 & 1 & 60.0 & 15.0 & & & .8 & 1.7 & .0 & $1.0 *$ \\
\hline 415 & & 1 & 2 & 73.0 & 15.0 & & & 1.0 & 1.9 & 1.0 & $1.1 *$ \\
\hline 417 & & 1 & 2 & 39.0 & 14.8 & 2.4 & 2.1 & 3.1 & 1.5 & 5.3 & 1.5 \\
\hline 417 & & 1 & 1 & 63.1 & 14.5 & -11.0 & 3.0 & 6.5 & 1.6 & 1.1 & $.5 *$ \\
\hline 421 & & 2 & 1 & 37.0 & 8.5 & .6 & 1.6 & 1.5 & 1.0 & 2.0 & 1.0 \\
\hline 423 & & 1 & 2 & 38.0 & 12.0 & & & 2.5 & 1.2 & .4 & $1.0 *$ \\
\hline 425 & & 1 & 1 & 83.0 & 16.0 & & & 2.3 & 1.8 & -1.3 & $1.0 *$ \\
\hline 425 & & 3 & 1 & 41.0 & 11.0 & 7.1 & 2.0 & 5.8 & 1.3 & 1.3 & $1.1 *$ \\
\hline 127 & & 1 & 2 & 71.2 & 8.3 & .8 & 1.6 & 3.3 & 1.0 & 2.8 & 1.0 \\
\hline 427 & & 3 & 1 & 100.3 & 16.1 & -4.7 & 2.6 & B.8 & 1.5 & .8 & $.5 *$ \\
\hline 429 & & 2 & 1 & 30.1 & 11.3 & 1.6 & 1.8 & -.3 & 1.5 & 3.9 & 1.2 \\
\hline 431 & & 1 & 2 & 35.4 & 15.8 & -1.3 & 1.0 & 3.6 & 1.4 & 4.6 & 1.4 \\
\hline 431 & & 3 & 1 & 129.3 & 22.2 & -12.1 & 3.2 & 10.4 & 2.3 & 1.4 & $.8 *$ \\
\hline 435 & & 1 & 1 & 37.1 & 14.0 & -1.6 & 2.1 & 1.5 & 1.3 & 7.5 & 1.5 \\
\hline 435 & & 3 & 1 & 106.3 & 20.2 & 7.9 & 2.3 & 11.5 & 2.2 & -.0 & $.7 *$ \\
\hline 437 & & 1 & 2 & 37.2 & 13.8 & 2.3 & 2.4 & 2.1 & 1.6 & 6.0 & 1.6 \\
\hline 137 & & 2 & 1 & 37.0 & 13.0 & & & 3.1 & 1.5 & -.9 & .9 \\
\hline 437 & & 3 & 1 & 77.9 & 18.5 & -10.2 & 3.2 & 7.1 & 1.8 & 1.3 & .6 \\
\hline 141 & & 3 & 1 & 30.1 & 13.4 & -3.8 & 2.1 & .4 & 1.5 & .1 & $.5 *$ \\
\hline 441 & & 7 & 1 & 40.7 & 12.4 & -.7 & 2.0 & 3.6 & 1.3 & 3.7 & 1.4 \\
\hline 443 & & 2 & 1 & 55.7 & 14.0 & 3.2 & 2.5 & 5.8 & 1.2 & 8.0 & 1.4 \\
\hline 445 & & 3 & 1 & 34.0 & 11.0 & & & 1.2 & 1.8 & -.3 & $1.0 *$ \\
\hline 14? & & 2 & 1 & 37.0 & 14.0 & & & 2.7 & 1.5 & .0 & $1.1 *$ \\
\hline 447 & & 3 & 1 & 31.4 & 14.3 & -4.8 & 2.3 & 5.0 & 1.6 & .7 & $.6 *$ \\
\hline 447 & & 9 & 1 & 89.3 & 13.9 & -5.5 & 2.6 & 9.2 & 1.4 & .8 & .5 \\
\hline 449 & & 3 & 1 & 38.0 & 11.0 & 1.0 & -.1 & 3.4 & 1.3 & 2.7 & $.9 *$ \\
\hline 451 & & 1 & 1 & 33.7 & 13.4 & 1.0 & 2.0 & 2.5 & 1.5 & 7.0 & $1.4 *$ \\
\hline 453 & & $\mathbf{3}$ & 1 & 47.8 & 17.5 & 1.0 & 2.0 & 2.3 & 1.6 & 7.0 & 1.6 \\
\hline 455 & & 2 & 1 & 31.0 & 2.6 & 7.2 & .5 & 5.4 & .3 & .3 & $.2 *$ \\
\hline 457 & & 1 & 1 & 38.9 & 12.0 & 3.3 & 1.5 & .8 & 1.4 & -.0 & $.5 *$ \\
\hline 457 & & 3 & 1 & 105.2 & 16.4 & -3.0 & 2.6 & 11.2 & 1.8 & .7 & $.6 *$ \\
\hline 477 & & 1 & 1 & 30.1 & 14.7 & 3.1 & 2.1 & 2.3 & 1.6 & 5.6 & 1.7 \\
\hline 479 & & 1 & 2 & 52.0 & 11.6 & .0 & 2.2 & 1.2 & 1.1 & 6.9 & 1.2 \\
\hline 479 & & 3 & 1 & 33.7 & 11.3 & 2.7 & 1.5 & 1.5 & 1.2 & 1.5 & 1.2 \\
\hline 483 & & 1 & 2 & 44.3 & 14.1 & -1.5 & 1.9 & 4.8 & 1.6 & 5.2 & 1.3 \\
\hline
\end{tabular}


TABLE 7. (contd)

\begin{tabular}{|c|c|c|c|c|c|c|c|c|c|c|c|}
\hline STAKE & DIstance & \multicolumn{2}{|c|}{ SAMPLE } & \multicolumn{2}{|c|}{ THORIUK } & \multicolumn{2}{|c|}{ RADIUY } & \multicolumn{2}{|c|}{ LEAD } & \multicolumn{2}{|c|}{ URANIUN } \\
\hline no. & METERS & TYPE & REP. & LEVEL & ERROR & LEVEL & ERROR & LEVEL & ERROR & LEVEL & ERROR \\
\hline 483 & & 3 & 1 & 76.2 & 14.5 & -.3 & 2.2 & 1.3 & 1.5 & 7.2 & 1.6 \\
\hline 485 & & 3 & 1 & 35.1 & 14.1 & 3.7 & 2.3 & .0 & 1.6 & 6.1 & 1.5 \\
\hline 487 & & 2 & 2 & 54.5 & 14.7 & -9.9 & 2.3 & 2.5 & 1.5 & 12.9 & 1.6 \\
\hline 487 & & 3 & 1 & 37.1 & 11.7 & -6.0 & 2.1 & 4.1 & 1.2 & 8.0 & 1.2 \\
\hline 489 & & 3 & 1 & 64.4 & 14.3 & 5.7 & 2.2 & 6.3 & 1.4 & 5.2 & 1.4 \\
\hline 489 & & 9 & 1 & 123.8 & 16.9 & 2.0 & 3.1 & 11.9 & 1.7 & 12.4 & 1.7 \\
\hline
\end{tabular}




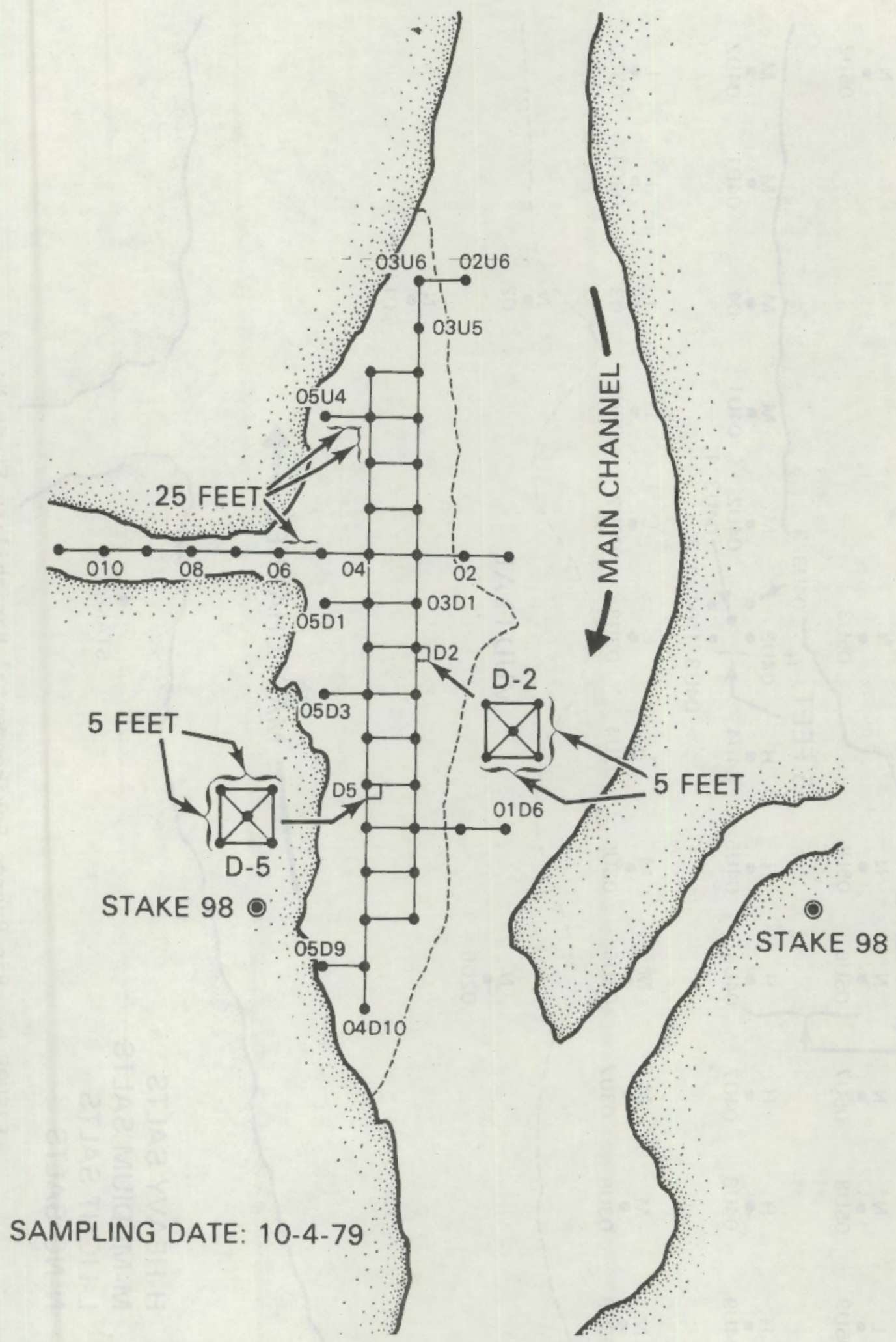

FIGURE 5. Rio Puerco Environmental Variability Study No. 1 


\section{MAIN BANK}

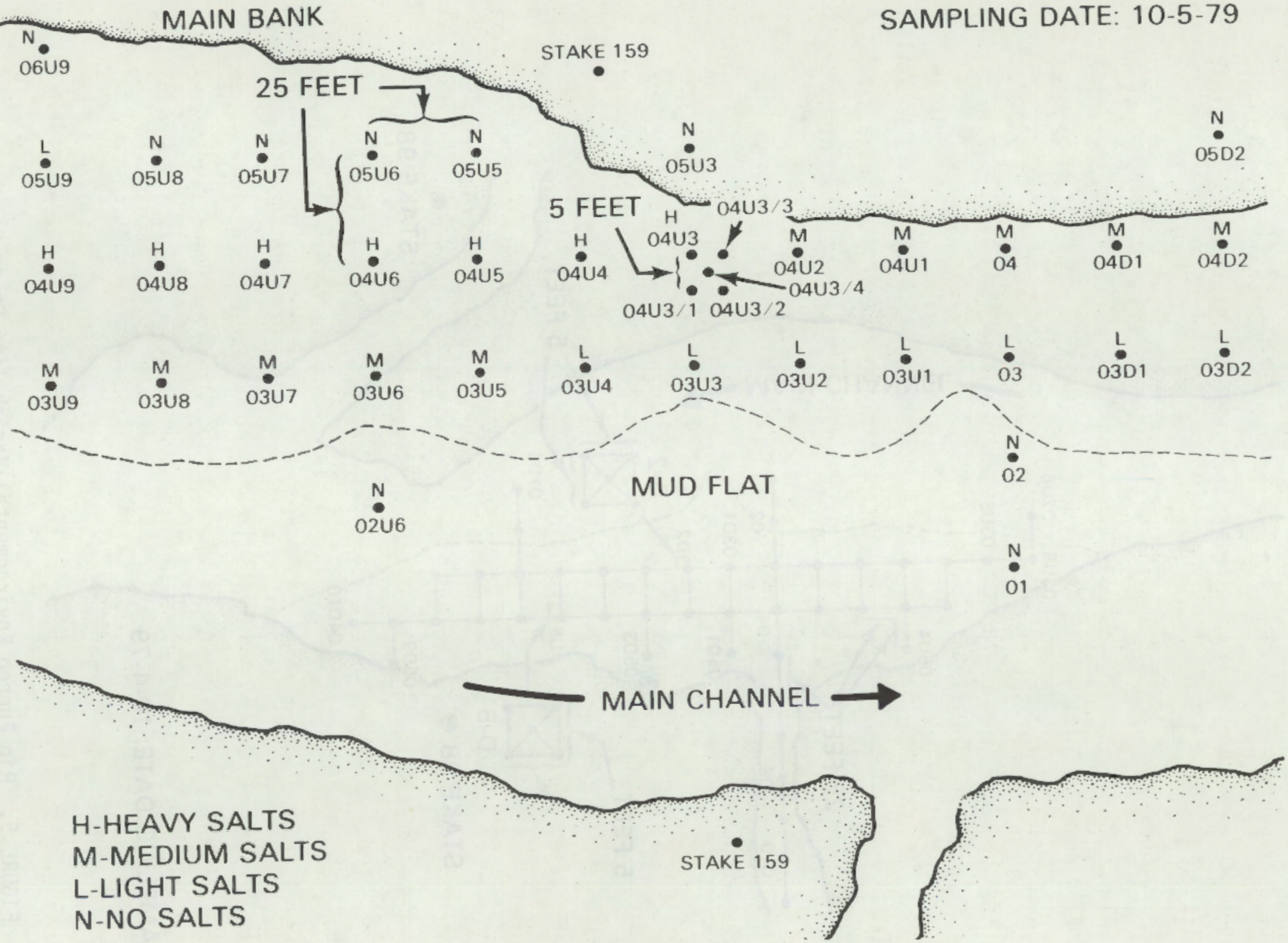

FIGURE 6. Rio Puerco Environmental Variability Study No. 2 
these sites can be expressed as either "within-sampling-site" or "betweensampling-sites" variability. Within-sampling-site variability refers to that variability calculated for replicate samples obtained from the 5-ft grids. Between-sampling-sites variability refers to that calculated between the 25-ft grid sites. The pooled within-sample-site standard deviation from the replicate samples was $35 \mathrm{pC} / \mathrm{g}$ (including the contributions of counting error) for the ${ }^{230}$ Th concentration. The between-sampling-locations standard deviation calculated from all of the ${ }^{230}$ Th data for these grid sampling patterns was $20 \mathrm{pCi} / \mathrm{g}$. Since the "within" standard deviation is much larger than the "between" standard deviation, we can assume that no significant spatial pattern can be found in these aata. Thus, the variability that is seen in these sandbar grid samplings seems to represent the inherent random error of sampling in the Rio Puerco arroyo.

\section{EVALUATION OF BACKGROUND SAMPLES}

The Rio Puerco arroyo crosses through geological areas which may contain significant uranium ore bodies or which may receive alluvial drainage from uranium ore formations or from current or former uranium mining sites. This situation creates the possibility whereby elevated concentrations of ${ }^{230} \mathrm{Th}$, and pernaps the other radionuclides of concern, may be present simply as part of the existing environment. An examination of the data from the Rio Puerco monitoring survey shows that only a single background sample collected at stake No. 201 exceeds the $30 \mathrm{pCi/g}{ }^{230}$ Th cleanup criteria, having a ${ }^{230}$ Th concentration of $34 \mathrm{pCi} / \mathrm{g}$. The background soil samples data are listed in Table 6 . The background concentrations of ${ }^{230}$ Th that are seen along the length of the arroyo are highly variable, ranging from -18 to $34 \mathrm{pCi} / \mathrm{g}$ with a mean background concentration of $0.87 \mathrm{pCi} / \mathrm{g}$. These data suggest that the past geochemical history of the soil samples may influence the thorium concentrations found, and show that background levels do vary along the length of the arroyo. (While this variability is quite large, much of this is due to the short counting time utilized and the accompanying large counting error. For example, only one ${ }^{230} \mathrm{Th}$ value is significantly less than zero at the $95 \%$ confidence interval.) 
One potential problem with the interpretation of the background sample data is related to the locations from which these samples were obtained. Due to the possibility of contamination of any sediments within the arroyo by the tailings pond solution, all background samples were obtained from soil locations near, but not in, the arroyo. The samples were generally collected at-grade within 50 to 100 yd of each side of the arroyo. As such, these samples probably represent soil materials very similar to what is eroded from the surrounding land and transported into the stream bed. However, very different mechanical forces, i.e., wind rather than water flow, affect the soil errosion. Any effect that these differences have on the surface soil and sediment mineralogies and natural radionuclide concentrations is not known.

It is clear from the above discussion that samples containing, for example, $35 \mathrm{pCi} / \mathrm{g}$ of ${ }^{230}$ Th may have an input of the tailings-pond-derived thorium ranging from only a few $\mathrm{pC} i / g$ to perhaps nearly the total thorium concentration found, depending upon the background level found in that particular area. Because of this potential difficulty in differentiating background versus tailings-derived thorium for any given segment of the stream bed, it is recommended that the most equitable means of establishing a cleanup criteria is to establish a criteria which is based upon a given concentration of ${ }^{230} \mathrm{Th}$ above a background level for that particular sample region. The rationale behind this approach is that the majority of the natural $230^{\text {Th }}$ is bound within the soil matrix material and, as such, does not represent a readily availabie fraction for movement in the environment or within an animai or human system. The contamination-derived thorium, on the other hand, will nearly all be associated with the surfaces of soil particles and likely is much more readily available for movement in either the environment or in an organism. Additional geochemical investigations of background and contaminated soil samples are required to address this question more adequately and to assist in establishing more accurate estimates of the average background ${ }^{230}$ Th concentrations along the entire Rio Puerco arroyo.

ESTIMATION OF ${ }^{230}$ Th INVENTORY

The first terrace. second terrace, and core samples data that are listed in Appendix $B$ were used to estimate a total inventory of ${ }^{230}$ Th along the 
length of the arroyo from the breech of the tailings pond dam through Stake No. 491. The ${ }^{230}$ Th inventory estimate presented here is only approximate, as of September, 1979, due to the considerable variability of the thorium concentrations, the varying background ${ }^{230}$ Th concentrations previously discussed, and the fact that only limited statistical treatment of the data was performed. The estimation was performed as described below.

For that portion of the arroyo for which sampling sites were surveyed onto the large UNC maps, these maps were used to estimate actual surface areas within the arroyo. Width measurements were made at each sampling point (odd numbered stakes) and the distance along the arroyo between even numbered stakes was measured. Then these two quantities were multiplied to get a surface area approximation. For the remainder of the arroyo, Stakes No. 306 through 491 , it was assumed that the stakes are $500 \mathrm{ft}$ apart and that the arroyo is $150 \mathrm{ft}$ wide. This width was determined from copies of smaller EPA aerial photographs which have a scale of approximately $200 \mathrm{ft} / \mathrm{cm}$ on the photos. Several width measurements were taken on each photo in this lower portion of the arroyo, and these measures were then averaged. This average was then rounded up to $150 \mathrm{ft}$ to give a somewhat conservative (large) estimate of arroyo surface area. These areas were multiplied by the 2-in. depth of the sampling device, and this volume was multiplied by the average of all terrace concentrations and the density of the samples to get an inventory for each approximately 1000-ft section of arroyo. These were summed for the entire arroyo to get the total ${ }^{230}$ Th inventory of $4.9 \mathrm{Ci}$. A background ${ }^{230}$ Th inventory has been calculated in a similar manner to be $0.30 \mathrm{Ci}$.

Some discussion is necessary on the rationale and meaning of a "surface" inventory. The spill did soak into the arroyo sediments. The data from the three-foot core samples that were collected at distances approximately one mile apart throughout the arroyo indicate that the surface samples alone do not provide a complete view of the distribution of ${ }^{230}$ Th in the arroyo. Cores taken at Stakes No. 31, 51, 71, 81, 131, 141, 201, 231, 271, 291, 311, and 451 all show evidence of measurable downward migration of ${ }^{230} \mathrm{Th}$. These represent 12 of the 49 locations where sediment cores were obtained. However, since core samples were taken only at every tenth stake number (every fifth sampling 
site), a considerable extrapolation is needed to use the core data. In spice of the recognized limitations of this approach, ${ }^{230}$ Th inventories in the first, second, and third foot of the arroyo sediments have been calculated. These calculations show $18.7 \mathrm{C} i$ in the first foot of sediments, $11.1 \mathrm{Ci}$ in the second foot of sediments, and $1.5 \mathrm{Ci}$ in the third foot of sediments. This striking difference in thorium content with depth suggests that much of the ${ }^{230}$ Th has been retained in the top two feet of the sediments. The inability to provide an accurate estimate of the background ${ }^{230} \mathrm{Th}$ in the arroyo sediments limits the interpretation of these oata.

While a surface inventory does not represent all of the material lost at the break of the dam, it does represent the source of possible exposure to humans and animals using the arroyo. Furthermore, by defining "surface" as the first two inches of depth, there is immediate agreenent with the standardized sampling tools. This agreement, in turn, allows the use of all the terrace aata without any reservations on the data interpretation. An examination of the data set and codes for type of sample will show that there are samples at each sampling site only for the terrace data. These factors and the fact tnat the sampling scheme was not originally designed for estimating inventory, dictate that calculation of a "surface" inventory provides the most reasonable retrospective use of the data.

\section{EVALUATION OF RIO PUERCO SAMPLING STRATEGY}

The basic philosophy of the Rio Puerco sampiing protocol which was established by the NMEID was to collect samples every $1000 \mathrm{ft}$ along the watercourse beginning at the point at which the spill entered the pipeline arroyo. At these sampling sites several different types of samples were taken: individual streambed terraces, around pools or concentrated areas, core samples, background samples, and samples of any visible salt deposits. The natural variations in the morphology of the stream mandate that some types of samples were not taken at each site.

The adequacy of this sampling protocol can be evaluated on two different levels, first with regard to the information available at the time of the sampling, and second with regard to what we now know about the Rio Puerco 
arroyo. In addition, it must be emphasized that any sampling plan needs to be evaluated with respect to very specific goals. The statement of the goals must contain precise definitions, give designated or desired error levels, and an estimate of the randomness that will be allowed in the data. Such a specific statement of acceptable statistical parameters was not produced during the short interval of time between the July 16, 1979, spill and the major sampling which was conducted September, 1979. From this standpoint, therefore, it is very difficult to evaluate whether or not the goals of the sampling program were met.

The sampling program was established by the NMEID in response to a need to provide very rapid sampling and sample analysis for an overall evaluation of the state of the arroyo. Given the urgency of this situation and the relatively short time available for planning, the sampling plan which was devised and used appears to be a good one. It contains the essential elements found in the typical survey-type investigations of spatially varying phenomenon. However, after a retrospective view of the data it is possible to identify some inadequacies. Equally spaced sampling sites as were designated in this survey are not necessarily a desirable feature in a sampling plan, since most statistical analysis procedures can deal adequately with variable spacing. The locations of the sampling sites should have been influenced by the geographical features of the arroyo. It would have been desirable to choose and classify sampling sites on the basis of geographical or morphological features such as meander of the arroyo, straightness of a section of the arroyo, depth of the water, or other features, since these might be important factors controlling the translocation of the contamination. An examination of the aerial maps of the arroyo indicate that many features on which sampling sites could be keyed do appear between the established sampling points. Also, there apparently is a small-scale structure to the arroyo that the sampling protocol missed because too few sampling locations were used. In developing the sampling protocol, however, it is likely that the economic considerations involved did limit the number of sampling sites selected. A reasonable compromise would have been to have increased the number of sampling locations in a few "typical" sections of 
the arroyo in order to describe the spatial variability well and then to decrease the number of sampling locations in similar sections of the arroyo.

EVALUATION OF VERIFICATION SAMPLING PLAN AND PROPOSEO CLEANUP CRITERIA

Dr. Thomas E. Buhl of NMEID in his letter to Mr. Hubert Miller of the U.S. NRC dated April 12, 1980, has proposed some revised Rio Puerco cleanup cri.. teria. (Please refer to Appendix F for a copy of the proposal by Dr. Buhl.) The criteria proposed are statistically simple and easy to apply. This proposal suggests the use of a $67 \%$ confidence level rather than the commonly used $95 \%$ confidence level. This choice of confidence level is statistically arbitrary, and there is no statistical or mathematical reason for the use of any particular level. With the range of thorium data values and counting errors stated in Dr. Buhl's letter, a $67 \%$ confidence level with a limit of $60 \mathrm{pC} i \mathrm{~g}$ is approximately equivalent to a $95 \%$ confidence level with a limit of $80 \mathrm{pC} / \mathrm{g}$. The use of a one-sided upper confidence level as indicated by addition of the standard error to the mean but no subtraction from the mean is standard and appropriate for this type of situation. (It should be noted that dividing the standard error by two yields a $69 \%$ rather than a $67 \%$ confidence level under the assumption of a normal distribution.)

The new criteria do not place any control on the sample size to be used. This results in a situation of no control of the second type of statistical error, that of concluding that radionuclide levels are within the limit when in fact they are high. The $67 \%$ confidence level controls the first type of statistical error, that of concluding that radionuclide levels are high when in fact they are within the limits. Because a large sample size results in a small standard error, a large sample could allow the mean values to be very close to the revised 1 imit of $60 \mathrm{pCi} / \mathrm{g}$ even when some members of the sample are much higher. In other words, more information (samples) is needed to make a decision when mean values are closer to the limit than when mean values are much lower (or higher) than the decision point. Small samples, on the other hand, would allow many of the second type of statistical errors, that of 
concluding that a mean value is less than the limit when in fact it is slightly higher. Therefore, the sampling criteria should state a minimum number of samples to be collected and analyzed.

The use of a stated level of radionuclide inclusive of background as the concentration limit or decision point $\left(60 \mathrm{pCi} / \mathrm{g}\right.$ for ${ }^{230} \mathrm{Th}$, for example) elininates the need for information about background levels in the decision-making process. A somewhat more common statistical procedure would be to state some allowable deviation above background levels, thus including the background information in the overall statistical analysis. While there is nothing statistically invalid in not using the background levels, it is not a common way of analyzing data. Given that there were less background data collected during the Rio Puerco sampling program than radionuclide data, the use of the background inclusive radionuclide level in the decision selection is perhaps a statistically preferred scheme. The lack of background information for all sampling points would otherwise require more extensive background sampling or the use of some type of interpolation methodology for those sampling locations for which background data are not available.

Examination of the aerial survey maps indicates that the survey stakes are not an accurate indication of streambed distances, at least for the first 305 of the 491 stakes for which survey data are available. Since it would be a major task to identify 1000-ft sections on the maps or to return to the arroyo for measurements, and since no maps with staked locations exist for the arroyo beyond Stake No. 305, the area criteria should be stated in terms of stakes rather than feet. The 1000-ft criteria should be changed to be the distance between alternate stakes.

The criteria speak of using 1000-ft sections of the arroyo for averaging purposes but do not mention how this section is to be chosen. Most of the samples were taken only at the odd-numbered stakes. This sampling methodology results in two distinctly different possible situations for computing area averages. If the areas are chosen to be between alternate even-numbered stakes, as would occur if one starts with Stake No. 0 , then data would be available for oniy the intermediate odd-numbered stakes. In this case the averages for that odd-numbered stake would be used as the average for the 
entire area. However, if the areas are chosen to be between alternate oddnumbered stakes, then data exist only at the boundaries or ends of the area, and an average of the two boundary averages would be used. A third alternative is to use overlapping areas starting with each stake and using both kinds of averages mentioned above. This alternative would be statistically troublesome since the two kinds of averages have different characteristics and distributions. Using an area criteria of $1000 \mathrm{ft}$ rather than a criteria of alternate stakes would be equivalent to this third alternative because of the lack of association of stake numbers with actual arroyo streambed distances. The criteria should specify exactly how the areas are to be chosen so that conflict cannot arise from different investigators using different methods. Sampling at the miapoint of the section is the easiest computationaliy and is a good way from the statistical standpoint as well.

The proposed criteria appear to ignore the information which is contained in the counting error data. The use of a simple standard error of the mean indicates that one is to calculate the standard deviation of the several replicates for each sample type at each collection point and divide by the square root of the sample size. This is a between-samples error measurement. Whan this is used alone, the within-samples error information contained in the counting error data is ignored. It is possible to use both the within-samples and the between-samples errors in a rather elegant manner to calculate a total standard error. This total standard error can then be used to determine the $67 \%$ confioence level as before. The counting error is a measure of the precision of the corresponding sample value. The more precise ones should be considered more important than the less precise sample values. A statistical way of achieving this weighting is to use the inverse of the variances (counting errors squared) as the weighting factors. For the weighted means and corresponding total standard errors advanced statistics texts (Brownlee 1965) give the following formulae:

$$
\text { mean }=\frac{\Sigma y / v}{\Sigma 1 / v}
$$




$$
\text { standard error }=\frac{1}{\sum 1 / v+(\text { B.S.E. })^{2}}
$$

where

$$
\begin{aligned}
y= & \text { data values } \\
v= & \text { corresponding variances } \\
\text { B.S.E. = } & \text { (between-samples standard error)--the common standard devia- } \\
& \text { tion calculated from the data values then divided by the } \\
& \text { square root of the sample size. }
\end{aligned}
$$

These formulae should be used to calculate weighted means and standard errors because they use the counting error information to adjust for the variation in precision between replicate samples as well as including both between-sample and within-sample errors.

Number of Sample Replicates

As stated above, a determination is needed of the number of sample replicates for cleanup verification at each sampling site. The statistical theory which relates to these calculations is that of specifying a confidence interval about a mean value. This statistical theory requires estimates of the mean and standard deviation and a specification of the desired accuracy. The mean and standard deviation were calculated from all of the Rio Puerco data as described below, and the accuracy was arbitrarily selected to be $100 \%$ of the average ${ }^{230}$ Th concentration. The thorium mean value of all first and second terrace data over the entire length of the arroyo is $24.1 \mathrm{pCi} / \mathrm{g}$. This was determined by first calculating a weighted mean for each sampling site (using the counting error as the weighting factor), then calculating the arithmetic average of these weighted means over the length of the arroyo.

A total variance was computed by summing the individual components of the variance (counting error squared). First, a pooled variance for each 1000-ft (or, more accurately, every two sampling stakes) section of the arroyo was computed. The weighted mean ${ }^{230}$ Th concentration variances for the first and second terrace aata were pooled for each section by averaging these variances. This procedure is equivalent to summing variances and dividing by the total 
degrees of freedom under the assumption that each mean has one degree of freedom, and this results in a variance that is a measure of the accuracy of individual mean values. The between-means variance was then calculated for each segment by calculating the ordinary standard deviation of the mean values associated with each segment. These two variances were summed to get a total within-segment variance. These variances were then pooled by averaging over the length of the arroyo.

The sections of the arroyo used for this determination of variance were taken to be the data from Stakes No. 1 and 3,5 and 7,9 and 11, etc. Avoiding overlapping sections ( 1 and 3,3 and 5,5 and 7 , etc.) eliminates some troublesome correlations that have to be accounted for in the computations. Using sections defined from alternate numbered stakes rather than overlapping stakes allows the final variance estimate to include good estimates of both withindata-values and between-data-values components of error. The estimate of the within-section standard deviation is $18.3 \mathrm{pCi} / \mathrm{g}$ for ${ }^{230} \mathrm{Th}$ in samples from terraces one and two. There were 119 segments of arroyo used in this determination. Most of the data from the last 20 stakes were not used because of missing values.

An accuracy of $100 \%$ of the mean value of $24.1 \mathrm{pCi} / \mathrm{g}^{230}$ Th can be interpreted in two ways: 1 ) as \pm 24 for a confidence interval ranging from 0 to $48 \mathrm{pCi} / \mathrm{g} 230 \mathrm{Th}$, or 2 ) as \pm 12 for a confidence interval ranging from 12 to $36 \mathrm{pCi} / \mathrm{g}^{230} \mathrm{Th}$. Calculations based on both interpretations are given below for an assumed $95 \%$ confidence level. The formula for the required number of samples based upon the statistical theory of confidence intervals about a mean value is:

$$
N=\left|\frac{s t}{ \pm d}\right|^{2}
$$

where $s$ = estimate of the standard deviation

$t=$ the value of students- $t$ distribution for the $95 \%$ confidence level

$d=$ the width of the confidence interval

$N=$ number of samples. 
Since the value used for $t$ depends upon sample size as well as confidence level, some simple trial and error is needed to find $N$. The estimate of $s$ is 18.3 as given above. The two interpretations of the $100 \%$ of mean value for the accuracy results in values of $d$ of \pm 24 and \pm 12 . Then we get $N=5$ for $\pm 24 \mathrm{pCi} / \mathrm{g}$, and $\mathrm{N}=12$ for $\pm 12 \mathrm{pCi} / \mathrm{g}$.

There are three important statistical particulars that need emphasis. First, the specified accuracy and confidence level is achieved on the average over the many sections of the entire arroyo. There is no guarantee (without going to much more complicated statistical theory) that the accuracy and contidence hold true for any one individual section. Secondly, the width of the confidence interval will be \pm 12 or $\pm 24 \mathrm{pCi} / \mathrm{g}$ regardless of the mean value for any one section. For example, if \pm 12 is chosen as the definition of $100 \%$ of the mean and a particular section has a mean value of $12 \mathrm{pCi} / \mathrm{g}$, then the confidence interval is from 0 to $24 \mathrm{pCi} / \mathrm{g}$. One might be tempted to assume that \pm 12 around a mean of 24 allows one to use \pm 6 around a mean of 12 , but this would be an incorrect use of the statistics. Of course, a narrower confidence interval, for all values of the mean, can be achieved by using larger samples. The key point is that statistical theory needs a statement of accuracy in terms of absolute deviations from the mean rather than proportionat deviations from the mean. Finally, one should be careful to apply the definition of a confidence interval correctly. A $95 \%$ confidence interval of 12 to $35 \mathrm{pci} / \mathrm{g}$ means that $95 \%$ of such confiaence intervals will include the true value of the mean. It does not mean such things as being $95 \%$ sure that the true mean is within \pm 12 units of the estimated mean. 



\section{EXPERIENCE GAINED FROM THE RIO PUERCO ENVIRONMENTAL STUDY}

In several ways the ata gained from this investigation of the Church Rock tajlings dam breaching and subsequent flooding of the Rio Puerco arroyo may be valuable in addressing other environmental surveys of the distribution of many hazardous chemicals. Specifically, the sample collection, sample analysis, data analysis, and relationship of the time of the release to the time of the inventory sampling program are important considerations.

The sample collection techniques which used the 10.16-cm (4-in.) diameter by 5 -cm (2-in.) deep stainless steel die were very effective for obtaining surface soil/sediment samples. These surface data were supplemented by data obtained from the core samples. Twelve of the 49 locations where sediment cores were obtained showed evidence of measurable downward movement of $230_{\text {Th }}$. Nine of these locations are broad areas of the arroyo where the rate of flow of the tailings pond solution may have decreased, allowing somewhat greater verti. cal penetration than in the narrower stretches of the arroyo. Since these locations by no means represent all of the broad stretches of the Rio Puerco, additional unmeasured factors must be involved in causing the downward penetration of ${ }^{230}$ Th at these sites. Only three of these 12 locations showed higher 230 Th concentrations at some depth within the cores than either the first or second terrace sample concentrations. This suggests that the surface samp?ings alone do provide an adequate picture of the downstream distribution of the radionuclide contamination for this arroyo but do not present a complete picture.

The large surface area, planar intrinsic germanium detectors are extreme $1 y$ usefil for this type of investigation. The degree of spectral resolution and counting sensitivity they offer al low low limits of detection for ${ }^{210} \mathrm{~Pb},{ }^{226} \mathrm{Ra}$, $230 \mathrm{Th}$, and ${ }^{238} \mathrm{U}$ in relatively short counting intervals. The relatively large counting errors associated with the 10 w ${ }^{230}$ Th activity samples are due to the 1000-sec counting times used. If future investigations would allow the processing of fewer samples per day (e.g., 50 or less), the improved counting statistics obtained from longer counting times would allow some additional 
interpretation of the data and improve overall contamination inventory esti.. mates. It is recommended that the counting times be increased to at least $2000 \mathrm{sec}$.

The extreme heterogeneity in concentration distribution seen in the Rio Puerco is most likely typical of the variability that would be seen in other similar environments. This variability is probably related to geochemical and morphological features of the stream that were not identified in this investigation. As discussed above, clustered sampling in stream regions of representative morphological features should provide better estimates of spatial variability than does the uniform sampling used for the Rio Puerco. Clustered sampling is recommended for any future investigations of contamination distribution.

A difficulty in estimating the ${ }^{230}$ Th inventory arose because of the inabijity to differentiate between contamination-derived ${ }^{230}$ Th and natural background ${ }^{230}$ Th. Since the natural background may fluctuate along the length of the arroyo (based upon the variability observed for the samples discussed above), with present data it is not possible at any one point to define the background contribution to the total ${ }^{230}$ Th burden of a sample. This situation is quite likely to exist in streambeds in other uranium mining and milling areas. Further geochemical characterization of both ${ }^{230}$ Th-contaminated and uncontaminated sediment/soil samples is necessary to provide the data that can be used in this differentiation.

Approximately ten weeks passed between the time of the breaching of the tailings pond dam and the initiation of the intensive arroyo sampling. Two major rainfall events occurred during this time, and these certainly affected the distribution or redistribution of the contamination which had been deposited on the arroyo sediments. This movement of contaminated sediments served only to make more difficult the determination of ${ }^{230}$ Th inventory and distribution. Any future spills should be addressed with an intensive sampling and analysis program as soon as possible after the event occurs, preferably in less than one week. Sampling in this time frame combined with a rapid sample analysis and a more efficient sample identification system than was used for the Rio Puerco samples should permit a definition of the contamination 
distribution within 1 to $1-1 / 2$ months from the time of the release. This quick identification of potential problem regions of the stream would permit ameliorative action to begin within a reasonable time frame. 



\section{CONCLUSIONS}

The following conclusions are presented as a result of the investigation of the Church Rock uranium mill tailings pond dam failure:

1. On-site use of planar intrinsic germanium detectors provides adequate sensitivity and short analys is times for quantitation of $210 \mathrm{~Pb}$, ${ }^{226} \mathrm{Ra},{ }^{230} \mathrm{Th}$, and ${ }^{238} \mathrm{U}$ in sediments/soils.

2. The original sampling plan, which consisted of collecting surface soil/sediment samples at 1000-ft intervals, was adequate to determine tinose areas which were contaminated and required cleanup.

3. Clustering of samples within arroyo reaches defined by physical (morphological) features would provide a better estimation of the radionuclide spatial variability than did the samp?ing pattern which was used and witich was based on equal distance increments.

7. The revised Rio Puerco cleanup criteria proposed by the New Mexico Environmental Improvement Division are statistically adequate.

5. Concentrations of $210^{\mathrm{Pb}},{ }^{226} \mathrm{Ra}$, and $238 \mathrm{U}$ in samples throughout the length of the arroyo are not distinguishable from natural backgrouno concentrations.

6. Concentrations of ${ }^{230}$ Th range from background levels to levels elevated considerably greater than background. Plots of 230 Th concentrations versus distance from the tailings pond show the high variability. Therefore, a statistical smoothing function was applied to the data to facilitate its interpretation.

7. Seoiment samples from two site-variability studies indicate that there is considerable ${ }^{230}$ Th concentration variabjlity within even limited areas of the arroyo (i.e., 5-ft-square grids and 25-ft-square grids).

8. The concentrations of ${ }^{230}$ Th in the Rio Puerco show an apparent periodicity as a function of distance. This period is approximately $2.5 \mathrm{~km}$. 
9. The estimated total inventory of ${ }^{230}$ Th in the Rio Puerco from sampling Stakes No. 0 to 491 is $26.8 \mathrm{Ci}$, based on data from the upper two feet of the core samples. The inventory based on data from the first-terrace and second-terrace samples (upper two inches) is $4.9 \mathrm{Ci}$. The total inventory of ${ }^{230}$ Th background in the upper two inches of arroyo sediments is estimated to be $0.30 \mathrm{Ci}$.

10. Present inability to differentitate between natural background ${ }^{230}$ Th and contamination-derived ${ }^{230}$ Th prohibits a clear definition of the 230 Th inventory from the tailings pond solution.

11. Sediment samples from the Grand Canyon National Park show no detectable radionuclide levels in excess of normal background. 


\section{REFERENCES}

Brownlee, K. A. 1965. Statistical Theory and Methodology in Science and Engineering, Second Edition. John Wiley and Sons, New York, New York. $586 \mathrm{pp}$.

Chatfield, C. 1976. The Analys is of Time Series: Theory and Practice. Chapman and Hall. London. $257 \mathrm{pp}$.

Otnes, R. I. and L. Enochson. 1978. Applied Time Series Analysis: Basic Techniques. John Wiley and Sons. New York, New York. $444 \mathrm{pp}$.

Wogman, N. A., D. E. Robertson and R. W. Perkins. 1967. "A Large Detector, Anticoincidence-Shielded, Multi-Dimensional Gamma-Ray Spectrometer."

Nuclear Inst. Meth. 50:1-10. 

APPENDIX A

NEW MEXICO ENVIRONMENTAL IMPROVEMENT DIVISION

PROTOCOL FOR RIO PUERCO ENVIRONMENTAL SAMPLING 
APPENDIX A

NEW MEXICO ENVIRONMENTAL IMPROVEMENT DIVISION

PROTOCOL FOR RIO PUERCO ENVIRONMENTAL SAMPLING

"Surface soil samples will be taken at 1000-ft intervals in nonvegetated areas along the Rio Puerco and the 'Pipeline Arroyo' from the UNC dam to the New Mexico Arizona border. Surface sampling will be performed using a standardized cylindrical scoop of 10.16-cm (4-in.) diameter and 5-cm (2-in.) height. Soil mass from a particular sampling location must be at least 1000 grams (this will require compositing of four standardized soil samples). All samples should be appropriately sealed and labeled as soon as collected.

"At each sampling location three types of samples will be taken:

1. a sample from the first terrace above the streambed chosen in areas of expected contaminate deposition. Where possible, the samples will be composited from two samples, one from each side of the Rio Puerco/pipeline arroyo ("First terrace").

2. a similar sample from the second terrace above the streambed, if such a terrace exists ("second terrace").

3. a sample from an area where deposition is expected to be the highest, such as a side arroyo, impoundment area, inner bank of a bend, or area of yellow crystallization ("hot spot").

"This will generate roughly: (40 miles) $x$ (5280 ft/mile) $x$

(3 samples/1000 ft) approximately 600 samples.

"In addition, the following special sampling will be performed:

1. Background sampling

a. 20 separate $1000-g$ samples shall be collected in the pipeline arroyo upstream from the UNC mill to the UNC and KerrMcGee mine discharge points. These will define pre-spill conditions in the arroyo.

b. 20 separate $1000-g$ samples shall be collected within the main branch of the Rio Puerco at a sufficient distance upstream from the confluence of the Rio Puerco and pipeline arroyo to avoid any area contaminated by the spi]l. 
c. A 1000-g soil sample shall ve taken every mile along the Rio Puerco and pipeline arroyo between the UNC mill and the New Mexico-Arizona border. This sample shall be composited of two samples on each side of the arroyo. The samples shall be collected at a distance of between 50 and 100 yards from the Rio Puerco or pipeline arroyo cut bank to establish natural area background.

2. Soil profiles shall be collected across the width of the arroyo to establish the depth and lateral extent of the contamination. At each mile along the Rio Puerco and pipeline arroyo between the UNC mill and the New Mexico-Arizona line two 3-ft profjles shall be collected. The samples shall be collected with either a standardized coring device (such as PVC pipe), the standardized scoop plus trenching where possible, or an approved alternate method. The profile will consist of three 1000-g samples taken from the following depth intervals: 0 to $1 \mathrm{ft}$, 1 to $2 \mathrm{ft}, 2$ to $3 \mathrm{ft}$.

In addition, a 3-ft soil profile will be collected in at least five areas between the mill and the New Mexico-Arizona line where the spilled mill liquor backed up, such as the pinedale crossing, in areas identified as contaminated by the ARMS survey, or as requestea by the Environmental Improvement Division.

3. Two 1000-g surface soil samples will be taken with the standardized scoop at each location where a pool was located by UNC during their August 15 survey, as reported oy UNC on USGS topographic maps submitted to the EID. One sample shall be collected from the area of the pool where the water was deepest, and the other from the point of discharge from the pool to the stream.

"All soil samples shall be labeled according to the following standard notation:

- range, township, section, smallest quarter, middle quarter, largest quarter

- date of collection

- collector

- type of sample (if surface sample, indicate if background, first terrace, second terrace, hot spot, pool center, pool discharge; if profile sample, indicate if sample is nearer stream or nearer cut oank, and indicate depth interval) 
"Description of unusual features (such as presence of salts, etc.). For example, R16W T17N S3 NE1/4 SE1/4 SW1/4 means range 16W, township $17 \mathrm{~N}$, northeast quarter of the southeast quarter of the southwest quarter of Section 3.

"No other code shall be used without this sample identification. This program will call for roughly the following number of samples and analyses:

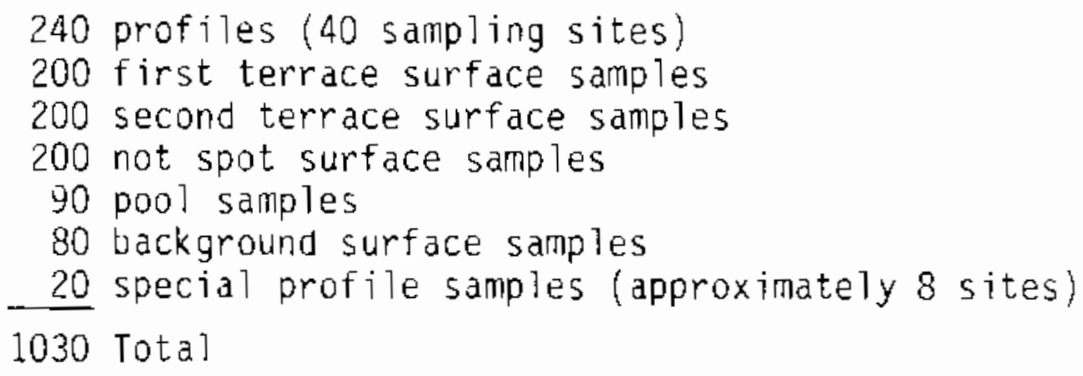

"This sampling will be completed by October 4, 1979." 

APPENDIX B

DATA FOR RIO PUERCO ENVIRONMENTAL SAMPLES 
APPENDIX B

\section{DATA FOR RIO PUERCO ENVIRONMENTAL SAMPLES}

KEY TO APPENDIX B SAMPLE CODING

Stake No.: Survey stake number

Level: Concentration in $\mathrm{pCi} / \mathrm{g}$

Error: Counting error.

\begin{tabular}{cl}
$\begin{array}{c}\text { Sample } \\
\text { Type }\end{array}$ & \multicolumn{1}{c}{ Identification } \\
\hline 1 & First Terrace \\
2 & Second Terrace \\
3 & Concentrated Area \\
4 & Pool Center \\
5 & Pool Discharge Point \\
6 & Background \\
7 & Core Near Stream \\
8 & Core Near Cut Bank \\
9 & Associated Salts
\end{tabular}

B. 1 


\begin{tabular}{|c|c|c|c|c|c|c|c|c|c|c|c|}
\hline \multirow{2}{*}{$\begin{array}{l}\text { STAKE } \\
\text { NO. }\end{array}$} & \multirow{2}{*}{$\begin{array}{l}\text { DISTANCE } \\
\text { HETERS }\end{array}$} & \multicolumn{2}{|c|}{ SAHPLE } & \multicolumn{2}{|c|}{ THOR IUH } & \multicolumn{2}{|c|}{ RALIUH } & \multicolumn{2}{|c|}{ LEAD } & \multicolumn{2}{|c|}{ URARIUH } \\
\hline & & TYPE & REF. & LEVEL & ERROR & LEVEL & ERROR & LEVEL & EKROR & LEUEL & ERKOF \\
\hline 0 & 0 & 1 & 2 & .0 & 8.7 & 1.8 & .2 & 1.5 & 1.0 & .0 & $1.0 *$ \\
\hline 0 & 0 & $t$ & 3 & 10.0 & 13.0 & 1.7 & .2 & 1.2 & 1.5 & -.9 & $1.2 *$ \\
\hline 0 & 0 & 2 & 1 & 12.0 & 12.0 & 1.8 & .2 & -.7 & 1.3 & 1.6 & $1.0 *$ \\
\hline 0 & 0 & 6 & 1 & -6.1 & 7.7 & 1.8 & .2 & .6 & .9 & -.8 & $.9 *$ \\
\hline 0 & 0 & 6 & 2 & 10.0 & 9.5 & 1.2 & .2 & 3.0 & 1.1 & .0 & $.9 *$ \\
\hline 0 & 0 & 7 & 1 & -7.3 & 8.2 & .8 & .1 & .7 & 1.0 & 1.1 & $1.0 \%$ \\
\hline 0 & 0 & 7 & 2 & 130.0 & 22.0 & 2.3 & .3 & 19.0 & 2.7 & 1.4 & $1.2 *$ \\
\hline 0 & 0 & 7 & 3 & 12.0 & 8.5 & 1.3 & .2 &.$\theta$ & .9 & 1.6 & $.8 *$ \\
\hline 0 & 0 & 8 & 1 & -3.6 & 8.3 & 1.3 & .2 & 1.5 & .9 & -.6 & $.8 *$ \\
\hline 0 & 0 & 8 & 2 & -17.9 & 12.0 & 1.1 & .2 & 1.5 & 1.4 & 1.2 & $.9 *$ \\
\hline 0 & 0 & 8 & 3 & 157.0 & 22.0 & 6.8 & .7 & 59.0 & 3.4 & 4.0 & $1.4 *$ \\
\hline 1 & 178 & 1 & 1 & 2.5 & 7.4 & 1.4 & .2 & 1.5 & .9 & -1.0 & $.9 *$ \\
\hline 1 & 178 & 2 & 1 & -13.9 & 10.0 & .8 & .1 & .8 & 1.5 & 1.4 & $1.0 *$ \\
\hline 1 & 178 & 6 & 1 & -1.7 & 11.0 & 1.4 & .2 & 4.6 & 1.6 & -.5 & $1.2 *$ \\
\hline 1 & 178 & 6 & 3 & 11.0 & 14.0 & 1.7 & .2 & 2.1 & 1.6 & -1.3 & $1.1 *$ \\
\hline 1 & 178 & 7 & 1 & 57.0 & 13.0 & 2,2 & .2 & 8.5 & 1.5 & -.1 & $1.1: k$ \\
\hline 1 & 178 & 7 & 2 & 35.0 & 15.0 & 1.2 & .2 & 6.3 & 1.7 & -.5 & $1.1: k$ \\
\hline 1 & 178 & 7 & 3 & 51.0 & 15.0 & 1.8 & .2 & 9.0 & 1.9 & -1.3 & $1.2 *$ \\
\hline 1 & 178 & 9 & 1 & 27.0 & 9.6 & 2.0 & .2 & 3.7 & 1.3 & .5 & $1.0 *$ \\
\hline 3 & 521 & 1 & 1 & 1.2 & 8.5 & 1.2 & .1 & 2.9 & 1.0 & -1.2 & $1.0 *$ \\
\hline 3 & 521 & 2 & 1 & 27.0 & 13.0 & .8 & .1 & 3.1 & 1.7 & -.3 & $.9 *$ \\
\hline 3 & 521 & 4 & 1 & 100.0 & 17.0 & 1.4 & .2 & 19.0 & 2.1 & .4 & $1.0 \%$ \\
\hline 3 & 521 & 4 & 2 & 200.0 & 19.0 & & & 11.0 & 1.8 &.$\Rightarrow$ & $1.0 *$ \\
\hline 3 & 521 & 9 & 1 & 62.0 & 16.0 & .0 & .4 & 9.0 & 2.1 & 1.2 & $1.2 *$ \\
\hline 5 & 835 & 1 & 1 & 13.0 & 8.7 & 1.4 & .2 & 1.5 & 1.0 & .0 & $.9: *$ \\
\hline 5 & B35 & 1 & 2 & 1.8 & 8.5 & .5 & .1 & 1.9 & 1.4 & -1.1 & $.8 *$ \\
\hline 5 & B35 & 2 & 1 & 4.9 & 9.4 & 1.4 & .2 & 5.4 & 1.1 & .0 & $1.0 *$ \\
\hline 7 & 1117 & 1 & 2 & 8.7 & 10.0 & .8 & .1 & .3 & 1.0 & -1.7 & $1.0 *$ \\
\hline 7 & 1147 & 1 & 3 & -5.2 & 12.0 & 1.3 & .2 & .6 & 1.3 & .6 & $1.1 *$ \\
\hline 7 & 1147 & 2 & 1 & 89.0 & 19.0 & 2.8 & .3 & 7.1 & 2.0 & -.7 & $1.2 *$ \\
\hline 7 & 1147 & 2 & 2 & 26.0 & 9.7 & .7 & .1 & 3.0 & 1.2 & .4 & $1.0 \%$ \\
\hline 7 & 1147 & 4 & 1 & 200.0 & 21.0 & 3.8 & .4 & 44.0 & 3.0 & .2 & $1.4 *$ \\
\hline 7 & 1147 & 9 & 1 & 1.8 & 15.0 & 1.0 & .2 & 7.1 & 1.8 & .6 & $1.0 \%$ \\
\hline 9 & 1471 & 1 & 1 & 7.1 & 11.0 & .9 & .1 & 2.3 & 1.7 & .2 & $.9: 1$ \\
\hline 9 & 1471 & 1 & 2 & 12.0 & 11.0 & 1.4 & .2 & 7.3 & 1.3 & .9 & $1.1 \%$ \\
\hline 9 & 1471 & 2 & 1 & 49.0 & 13.0 & 1.4 & .2 & 3.3 & 1.3 & .9 & $1.0 \star$ \\
\hline 9 & 1471 & 2 & 2 & 36.0 & 12.0 & 1.6 & .2 & 9.9 & 1.5 & .5 & $1.0 *$ \\
\hline 11 & 1754 & 1 & 1 & 34.0 & 15.0 & 1.9 & .2 & 1.9 & 1,8 & -.5 & $1.2 *$ \\
\hline 11 & 1754 & 1 & 2 & 12.0 & 11.0 & 1.8 & .2 & 7.0 & 1.3 & -1.0 & $1.1 *$ \\
\hline 11 & 1754 & 2 & 1 & 25.0 & 14.0 & 1.2 & .2 & 1.7 & 1.6 & -.1 & $1.1 \%$ \\
\hline 11 & 1754 & 2 & 2 & 12.0 & 10.0 & 1.1 & .2 & 2.7 & 1.2 & .9 & $1.0 *$ \\
\hline
\end{tabular}




\begin{tabular}{|c|c|c|c|c|c|c|c|c|c|c|c|}
\hline STAKE & DISTANCE & \multicolumn{2}{|c|}{ SAHPLE } & \multicolumn{2}{|c|}{ THORIUH } & \multicolumn{2}{|c|}{ RAIJUN } & \multicolumn{2}{|c|}{ LEAD } & \multicolumn{2}{|c|}{ URANIUN } \\
\hline No. & HETERS & TYYE & REP. & LEVEL & ERROR & LEVEL & ERROR & LEVEL & ERROR & LEVEL & ERROR \\
\hline 11 & 1754 & 6 & 1 & 25.0 & 12.0 & 1.1 & .1 & 2.2 & 1.6 & .9 & $1.1 *$ \\
\hline 11 & 1754 & 6 & 2 & -3.6 & 7.7 & 1.2 & .2 & 2.6 & .9 & .5 & $.9 *$ \\
\hline 11 & 1754 & 7 & 1 & -15.9 & 10.0 & 1.6 & .2 & 1.9 & 1.1 & .2 & $1.1 *$ \\
\hline 11 & 1754 & 7 & 2 & 19.0 & 17.0 & 3.0 & .3 & 3.3 & 1.8 & 2.2 & $1.4 \%$ \\
\hline 11 & 1754 & 7 & 3 & 8.7 & 8.7 & 5.2 & 1.0 & .7 & .9 & 1.1 & $1.0 *$ \\
\hline 11 & 1754 & 8 & 1 & -5.2 & 13.0 & 2.6 & .5 & .8 & 1.7 & -.5 & .94 \\
\hline 11 & 1754 & B & 2 & 16.0 & 13.5 & 2.1 & .6 & 1.2 & 1.6 & 2.4 & $1.0 *$ \\
\hline 11 & 1754 & B & 3 & -8.6 & 8.1 & 1.5 & .3 & .4 & 1.0 & .0 & $.9 *$ \\
\hline 13 & 2073 & 1 & 1 & 04.0 & 15.0 & 2.7 & .3 & 24.0 & 2.1 & .2 & $1.2 *$ \\
\hline 13 & 2073 & 1 & 2 & $-8 . B$ & 12.0 & 2.0 & .2 & 2.9 & 1.7 & -.3 & $1.0 *$ \\
\hline 13 & 2073 & 2 & 1 & 28.0 & 9.7 & 1.4 & .2 & 3.0 & 1.2 & .2 & $1.0 *$ \\
\hline 13 & 2073 & 2 & 2 & .0 & 9.7 & 1.9 & .2 & 2.2 & .9 & -1.3 & $1.0 *$ \\
\hline 15 & 2359 & 1 & 1 & 11.0 & 9.3 & .9 & .1 & 2.2 & 1.1 & -.3 & $.9 *$ \\
\hline 15 & 2359 & 2 & 2 & -6.1 & 9.2 & 1.1 & .2 & 2.6 & .9 & -2.6 & $1.0 *$ \\
\hline 17 & 2657 & 1 & 1 & 21.0 & 13.0 & 2.1 & .2 & 4.8 & 1.8 & 1.6 & $1.3 *$ \\
\hline 17 & 2657 & 1 & 2 & 92.0 & 15,0 & 1.6 & .2 & 16.0 & 1.8 & -.1 & $1.2 *$ \\
\hline 17 & 2657 & 4 & 1 & 14.0 & 14.0 & 2.0 & .2 & 4.1 & 1.9 & -.3 & 1.14 \\
\hline 19 & 2897 & 1 & 1 & 110.0 & 21.0 & 2.7 & .3 & 30.0 & 3.3 & 1.6 & $1.3 *$ \\
\hline 19 & 2897 & 1 & 2 & -3.6 & 7.5 & .7 & .1 & 1.4 & 1.0 & .9 & .74 \\
\hline 19 & 2897 & 1 & 3 & -10.9 & 10.0 & .2 & .1 & 1.9 & 1.4 & .8 & $1.0:$ \\
\hline 19 & 2897 & 3 & 1 & 270.0 & 22.0 & 1.1 & .2 & 11.0 & 1.6 & 2.0 & $1.3 *$ \\
\hline 19 & 2897 & 4 & 1 & -17.9 & 7.3 & .3 & .1 & 1.7 & .8 & .9 & $.7 *$ \\
\hline 19 & 2897 & 4 & 2 & -4.9 & 7.2 & .8 & .1 & .1 & .8 & 2.2 & $.9 t$ \\
\hline 19 & 2897 & 4 & 3 & 1.8 & 13.0 & 1.7 & .2 & 2.3 & 1.6 & -.3 & $1.0 \%$ \\
\hline 21 & 3255 & 1 & 1 & 14.8 & 10.2 & .7 & 1.8 & 3.2 & 1.1 & 4.0 & 1.0 \\
\hline 21 & 3255 & 1 & 2 & 44.3 & 16.1 & .6 & 2.2 & 11.0 & 2.3 & 7.5 & 1.6 \\
\hline 21 & 3255 & 6 & 1 & 2.5 & 6.8 & 4.3 & .6 & .7 & .0 & -.1 & $.4 *$ \\
\hline 21 & 3255 & 6 & 2 & -12.3 & 9.2 & -.7 & 2.2 & -.5 & 1.2 & .4 & $.4 *$ \\
\hline 21 & 3255 & 7 & 1 & 26.0 & 12.0 & 1.0 & 1.9 & -.3 & 1.0 & 2.7 & 1.3 \\
\hline 21 & 3255 & 7 & 2 & 4.9 & 7.2 & -2.0 & 1.7 & 1.6 & .9 & 4.1 & .9 \\
\hline 21 & 3255 & 7 & 3 & 11.0 & 11.0 & -.5 & 1.7 & .4 & 1.2 & 3.4 & 1.3 \\
\hline 21 & 3255 & a & 1 & 54.0 & 12.0 & .1 & 2.0 & 12.0 & 1.6 & 4.6 & 1.1 \\
\hline 21 & 3255 & 8 & 2 & 14.0 & 10.0 & 4.3 & 1.7 & 2.1 & 1.1 & 1.8 & 1.1 \\
\hline 21 & 3255 & 8 & 3 & 3.7 & 0.1 & 4.2 & 1.7 & 2.6 & .9 & 3.7 & 1.0 \\
\hline 23 & 3526 & 1 & 1 & B. 7 & 9.5 & 1.3 & 1.8 & 2.9 & 1.1 & 3.9 & 1.0 \\
\hline 23 & 3526 & 2 & 1 & 12.3 & 7.0 & .2 & 1.6 & .4 & .8 & 2.2 & .8 \\
\hline 25 & 3888 & 1 & 1 & 18.0 & 12.0 & 1.2 & .2 & 6.5 & 1.9 & 1.4 & $1.0 *$ \\
\hline 25 & उBBB & 2 & 1 & 5.3 & 10.0 & 1.0 & .1 & 2.3 & 1.5 & .6 & $.9 *$ \\
\hline 27 & 4140 & 1 & 1 & 23.0 & 10.8 & -3.9 & 1.6 & -6.4 & 1.8 & 3.3 & 1.2 \\
\hline 27 & 4140 & 1 & 2 & 30.1 & 15.5 & 1.5 & 1.8 & 7.7 & 1.7 & 6.8 & 1.6 \\
\hline 27 & 4140 & 9 & 1 & 85.0 & 14.0 & & & 15.0 & 1.8 & .7 & $1.0 \%$ \\
\hline
\end{tabular}




\begin{tabular}{|c|c|c|c|c|c|c|c|c|c|c|c|}
\hline STAKE & DISTANCE & SAH & PLE & THE & & & & & & UR & \\
\hline NO. & HETERS & TYPE & REF. & LEVEL & ERROR & LEVEL & ERROR & LEVEL & ERROR & LEVEL & ERROR \\
\hline 29 & 4430 & 1 & 1 & -7.0 & 12.0 & 2.0 & 1.8 & 1.7 & 1.6 & 2.5 & 1.2 \\
\hline 29 & 4430 & 1 & 2 & 16.1 & 8.3 & -1.8 & 1.7 & 2.2 & 1.1 & 3.4 & 1.0 \\
\hline 31 & 4704 & 1 & 1 & 16.0 & 9.0 & -3.4 & 1.6 & 1.2 & .9 & 3.0 & .8 \\
\hline 31 & 4704 & 1 & 2 & 12.4 & 10.8 & 4.0 & 1.6 & -.9 & 1.2 & 2.0 & 1.1 \\
\hline 31 & 4704 & 6 & 1 & 3.7 & 9.0 & 1.3 & .2 & .0 & 1.0 & -.6 & $1.1:$ \\
\hline 31 & 4704 & 6 & 2 & 3.7 & 7.3 & .8 & .1 & 1.7 & .9 & 1.4 & $.9 *$ \\
\hline 31 & 4704 & 7 & 1 & -4.8 & 7.6 & .4 & 1.7 & 2.5 & 1.0 & 4.0 & 1.0 \\
\hline 31 & 4704 & 7 & 2 & 47.8 & 15.9 & -.0 & 2.1 & 10.7 & 2.0 & 6.5 & 1.6 \\
\hline 31 & 4704 & 7 & 3 & 44.6 & 10.1 & 1.6 & 1.9 & 6.3 & 1.3 & 3.1 & 1.1 \\
\hline 31 & 4704 & $\theta$ & 1 & $2 B .3$ & 12.0 & -.8 & 2.0 & 5.0 & 1.5 & 5.5 & 1.3 \\
\hline 31 & 4704 & 8 & 2 & 34.7 & 11.8 & 2.6 & 2.0 & 5.6 & 1.2 & 4.0 & 1.0 \\
\hline 31 & 4704 & 8 & 3 & 14.0 & 13.0 & .9 & .2 & .6 & 1.6 & -.7 & $1.0 *$ \\
\hline 33 & 5045 & 1 & 1 & -6.9 & 12.3 & 2.4 & 2.0 & .4 & 1.4 & 2.8 & 1.4 \\
\hline 33 & 5045 & 2 & 1 & 24.7 & 10.4 & .2 & 2.0 & 8.0 & 1.4 & 6.3 & 1.1 \\
\hline 35 & 5338 & 1 & 1 & 19.0 & 12.0 & 1.1 & .1 & -.1 & 1.8 & .6 & $.9 *$ \\
\hline 35 & 5338 & 1 & 2 & 42.0 & 16.0 & 1.7 & .2 & .0 & 3.5 & -.9 & $2.3 *$ \\
\hline 35 & 5338 & 2 & 1 & 14.0 & 10.0 & 1.2 & .2 & 4.0 & 1.3 & .4 & $1.0 *$ \\
\hline 37 & 5616 & 1 & 1 & 27.0 & 12.0 & .8 & .1 & 1.0 & 1.6 & .4 & $1.0 *$ \\
\hline 37 & 5616 & 2 & 1 & 16.0 & 7.9 & .5 & .1 & 3.9 & 1.1 & 1.6 & $.8 *$ \\
\hline 37 & 5616 & 3 & 1 & 140.0 & 24.0 & 2.2 & .3 & 26.0 & 3.0 & .2 & $1.2 *$ \\
\hline 37 & 5616 & 3 & 2 & 36.0 & 12.0 & 1.4 & .2 & 5.5 & 1.2 & .7 & $.9 ;$ \\
\hline 37 & 5616 & 4 & 1 & -11.9 & 13.0 & & & 5.4 & 2.0 & -.1 & $1.2 *$ \\
\hline 39 & 5899 & 1 & 1 & 7.4 & 7.8 & .5 & .1 & 2.8 & .9 & -.6 & $.9 *$ \\
\hline 39 & 5899 & 3 & 1 & 34.0 & 16.0 & 1.7 & .2 & 0.6 & 2.3 & -.1 & $1 . t *$ \\
\hline 39 & 5899 & 3 & 2 & -19.9 & 11.0 & 1.4 & .2 & 3.5 & 1.3 & .9 & $1 . t *$ \\
\hline 41 & 6223 & 1 & 1 & 67.0 & 14.0 & 1.0 & .1 & 5.9 & 2.0 & .8 & $.9 *$ \\
\hline 41 & 6223 & 1 & 2 & 77.0 & 13.0 & & & 4.4 & 1.2 & .4 & $1.0 *$ \\
\hline 41 & 6223 & 3 & 1 & 20.0 & 8.2 & 1.1 & .2 & 3.9 & 1.1 & & $.9: *$ \\
\hline 41 & 6223 & 6 & 1 & 17.0 & 9.7 & 1.4 & .2 & 1.9 & 1.0 & .0 & $.9: *$ \\
\hline 41 & 6223 & 6 & 2 & 18.0 & 12.5 & .9 & .1 & 1.7 & 1.5 & -.7 & $1.0 *$ \\
\hline 41 & 6223 & 7 & 1 & 51.0 & 16.0 & 1.2 & .2 & B. 4 & 1.8 & 1.2 & $1.0 *$ \\
\hline 41 & 6223 & 7 & 2 & 14.0 & 9.7 & .8 & .1 & 1.9 & .9 & .9 & $.8 *$ \\
\hline 41 & 6223 & 7 & 3 & -34.9 & 13.0 & & & .0 & 1.0 & 1.0 & $1.1 *$ \\
\hline 41 & 6223 & 8 & 1 & 9.9 & 9.3 & 1.0 & .1 & 1.9 & 1.0 & -.1 & $.9 *$ \\
\hline 41 & 6223 & B & 3 & -13.9 & 10.0 & & & 2.7 & 1.3 & -.7 & $.8 *$ \\
\hline 43 & 6573 & 1 & 1 & 21.0 & 9.2 & .7 & .1 & 3.2 & 1.0 & -.6 & $.9 k$ \\
\hline 43 & 6573 & 1 & 2 & 13.6 & 10.0 & 4.4 & 1.9 & 1.9 & 1.0 & 2.8 & 1.0 \\
\hline 45 & 6839 & 1 & 2 & 3.5 & 11.0 & .5 & .1 & -.5 & 1.5 & .0 & $.9 *$ \\
\hline 45 & 6839 & 3 & 1 & 20.0 & 9.3 & .5 & .1 & .8 & .8 & -1.0 & $.9 *$ \\
\hline 47 & 7309 & 1 & 1 & 30.9 & 9.0 & -2.7 & 1.7 & 4.1 & 1.2 & 4.5 & 1.0 \\
\hline 47 & 7309 & 1 & 2 & -6.9 & 10.9 & 1,3 & 1.5 & .0 & 1.3 & 1.4 & 1.1 \\
\hline
\end{tabular}




\begin{tabular}{|c|c|c|c|c|c|c|c|c|c|c|c|}
\hline \multirow{2}{*}{$\begin{array}{l}\text { STAKE } \\
\text { NO. }\end{array}$} & \multirow{2}{*}{$\begin{array}{l}\text { JISTANCE } \\
\text { HETERS }\end{array}$} & \multicolumn{2}{|c|}{ SAHPLE } & \multicolumn{2}{|c|}{ THOR IUK } & \multicolumn{2}{|c|}{ RAUIUH } & \multicolumn{2}{|c|}{ LEAD } & \multicolumn{2}{|c|}{ UKANIUH } \\
\hline & & TYPE & KEP. & LEVEL & ERROR & LEUEL & ERKOR & LEVEL & ERROR & I_EVEL & ERKOR \\
\hline 47 & 7309 & 2 & 1 & 45.9 & 10.9 & 1.8 & 1.7 & 3.1 & 1.1 & 2.8 & 1.0 \\
\hline 49 & 7525 & 1 & 1 & 42.5 & 13.7 & -.1 & 2.0 & 6.7 & 1.8 & 6.3 & 1.3 \\
\hline 49 & 7525 & 1 & 2 & 103.0 & 19.0 & & & 10.0 & 2.0 & 2.0 & $1.2 *$ \\
\hline 49 & 7525 & 4 & 1 & 12.0 & 8.6 & & & 1.1 & .8 & 1.4 & $1.0 *$ \\
\hline 51 & 7872 & 1 & 1 & 79.0 & 16.0 & 1.8 & 2.9 & 6.6 & 1.2 & 10.0 & 1.2 \\
\hline 51 & 7827 & 1 & 2 & 31.0 & 11.0 & 1.1 & 1.8 & 3.0 & 1.0 & 3.0 & 1.1 \\
\hline 51 & 7827 & 2 & 1 & -3.4 & 12.0 & 1.9 & 1.7 & .0 & 1.3 & 3.2 & 1.3 \\
\hline 51 & 7827 & 6 & 1 & 8.9 & 13.0 & & & 1.5 & 1.6 & -2.6 & $1.1 *$ \\
\hline 51 & 7827 & 7 & 1 & 28.0 & 16.0 & & & 5.4 & 1.7 & 2.0 & .97 \\
\hline 51 & 7827 & 7 & 2 & 76.0 & 14.0 & 1.9 & 1.7 & B. 1 & 1.4 & .7 & .91 \\
\hline 51 & 7827 & 7 & 3 & .0 & 11.0 & -1.0 & 1.9 & 1.2 & 1.2 & 6.2 & 1.4 \\
\hline 51 & 7827 & 8 & 1 & 22.0 & 10.0 & -4.3 & 1.8 & 4.0 & 1.1 & 1.8 & 1.2 \\
\hline 51 & 7827 & 8 & 2 & 14.0 & 10.0 & & & 1.2 & 1.2 & 2.0 & 1.00 \\
\hline 31 & 7827 & B & 3 & -8.6 & 8.7 & 1.9 & 1.4 & -.0 & .9 & .6 & $1.9:$ \\
\hline 53 & 8163 & 1 & 1 & 64.0 & 13.0 & 3.3 & 2.0 & 9.2 & 1.4 & 4.5 & 1.3 \\
\hline 53 & 8163 & $\uparrow$ & 2 & 23.0 & 12.0 & 1.6 & 2.0 & 4.4 & 1.5 & 5.2 & 1.3 \\
\hline 53 & 8163 & 4 & 1 & 52.0 & 13.0 & & & 9.0 & 1.5 & 1.1 & $1.0 *$ \\
\hline 55 & 8473 & 1 & 1 & 1.8 & 10.0 & .9 & .2 & 4.2 & 1.5 & -.7 & $.9 *$ \\
\hline 55 & 8473 & 1 & 2 & 83.0 & 18.0 & 1.1 & .2 & 6.1 & 1.9 & 1.0 & $1.1 *$ \\
\hline 55 & 8473 & 4 & 1 & 25.0 & 12.0 & 1.4 & 1.5 & 5.9 & 1.7 & 1.0 & 1.11 \\
\hline 55 & 8473 & 4 & 2 & 16.0 & 12.0 & 1.1 & .2 & 4.2 & 1.8 & -.1 & $1.0 *$ \\
\hline 57 & 8002 & 1 & 1 & 0.9 & 10.0 & 1.2 & 1.7 & 1.5 & 1.3 & 3.3 & 1.0 \\
\hline 57 & 8802 & 1 & 2 & 53.0 & 13.0 & -.5 & 2.2 & 8.8 & 1.3 & 6.6 & 1.3 \\
\hline 57 & 8002 & 3 & 1 & 112.0 & 21.0 & 7.2 & 2.3 & 13.0 & 2.5 & .6 & $1.1 *$ \\
\hline 57 & 8802 & 4 & 1 & 5.0 & 9.3 & 2.0 & 2.1 & 2.6 & 1.1 & .2 & .41 \\
\hline 59 & 9064 & 1 & 1 & 21.0 & 13.0 & 1.6 & 2.1 & 5.8 & 1.7 & 4.8 & 1.4 \\
\hline 59 & 9064 & 1 & 2 & 6.2 & 9.5 & & & 3.4 & 1.1 & -.1 & .9 \\
\hline 61 & 9431 & 1 & 1 & -7.0 & 13.0 & 1.7 & 1.7 & 1.2 & 1.3 & 1.6 & 1.2 \\
\hline 61 & 9431 & 1 & 2 & 23.0 & 9.7 & -1.0 & 2.0 & 2.3 & 1.0 & 5.5 & 1.0 \\
\hline 61 & 9431 & 2 & 1 & 23.0 & 9.3 & 3.7 & 2.1 & 2.6 & 1.0 & 3.9 & 1.0 \\
\hline 61 & 9431 & 2 & 2 & 35.0 & 14.0 & -1.0 & 1.8 & 2.7 & 1.6 & 4.6 & 1.3 \\
\hline 61 & 9431 & 4 & 1 & 73.0 & 18.0 & 1.3 & .2 & 10.0 & 2.2 & -2.1 & 1.3 \\
\hline 61 & 9431 & 6 & 1 & -8.7 & 13.0 & & & 3.1 & 1.8 & 1.4 & $1.1:$ \\
\hline 61 & 9431 & 7 & 1 & -3.4 & 10.0 & 3.6 & 1.3 & 2.1 & 1.3 & 1.5 & 1.1 \\
\hline 64 & 9431 & 7 & 2 & 19.0 & 8.5 & -5.1 & 2.4 & 1.8 & 1.0 & .6 & $.4 *$ \\
\hline 61 & 9431 & 7 & 3 & -6.1 & 7.7 & & & .4 & .7 & .4 & 1.0 \\
\hline 61 & 9431 & 8 & 1 & 22.0 & 9.7 & 4.0 & 1.9 & 1.6 & 1.0 & 3.5 & 1.0 \\
\hline 61 & 9431 & 8 & 2 & 12.0 & 13.0 & 5.0 & 1.6 & 3.8 & 1.4 & -3 & 1.3 \\
\hline 61 & 9431 & 8 & 3 & -1.1 & 8.3 & 1.9 & 1.5 & 1.5 & .9 & 1.3 & .9 \\
\hline 63 & 9721 & 1 & 1 & 10.0 & 9.9 & .1 & 2.0 & 3.9 & 1.2 & 4.8 & 1.2 \\
\hline 63 & 9721 & $\uparrow$ & 2 & 35.0 & 11.0 & 3.3 & 1.7 & 4.3 & 1.1 & .7 & .91 \\
\hline
\end{tabular}




\begin{tabular}{|c|c|c|c|c|c|c|c|c|c|c|c|}
\hline STAKE & DISTAKCE & SAMI & LE & THO & & & & & & & \\
\hline NO. & AETERS & TYPE & REP. & LEVEL & ERROR & LEVEL & ERROR & LEVEL & ERROR & LEVEL & ERROR \\
\hline 63 & 9721 & 2 & 1 & 33.7 & 13.6 & -2.8 & 2.2 & 7.1 & 1.8 & 7.2 & 1.4 \\
\hline 63 & 9721 & 2 & 2 & 39.6 & 12.6 & .1 & 2.0 & 7.2 & 1.3 & 6.2 & 1.3 \\
\hline 65 & 9983 & 1 & 1 & 19.0 & 10.0 & 3.3 & 1.6 & 3.0 & 1.1 & -1.3 & .9. \\
\hline 65 & 9983 & 1 & 2 & 28.0 & 13.0 & .6 & .1 & 2.1 & 1.7 & .0 & $1.0 *$ \\
\hline 65 & 9983 & 2 & 1 & -5.2 & 14.0 & 2.9 & 1.6 & 2.7 & 1.7 & .0 & $1.2 *$ \\
\hline 65 & 9983 & 4 & 1 & 69.3 & 13.9 & -5.5 & 2.6 & 9.2 & 1.4 & .8 & $.5 *$ \\
\hline 67 & 10432 & 1 & 1 & 3.5 & 13.5 & .9 & 1.9 & 5.4 & 1.7 & 4.1 & 1.3 \\
\hline 67 & 10432 & 1 & 2 & 19.9 & 10.2 & .5 & 2.1 & 5.6 & 1.2 & 5.1 & 1.2 \\
\hline 67 & 10432 & 4 & 1 & 18.6 & 9.0 & $-2,7$ & 2.1 & 2.6 & 1.1 & .7 & $.5 *$ \\
\hline 67 & 10432 & 4 & 3 & -8.8 & 9.5 & 2.3 & 1.5 & 4.2 & 1.4 & .2 & $.8 *$ \\
\hline 69 & 10761 & 1 & 1 & 3.7 & 9.3 & -1.3 & 2.3 & 4.1 & 1.1 & .4 & $.4 *$ \\
\hline 69 & 10761 & 1 & 2 & 7.1 & 14.0 & -3.1 & 2.1 & 4.6 & 1.5 & .5 & $.4 *$ \\
\hline 69 & 10761 & 4 & 1 & 21.0 & 12.0 & 3.0 & 1.8 & 2.1 & 1.8 & .1 & $.5 *$ \\
\hline 69 & 10761 & 4 & 2 & 27.0 & 12.0 & -8.6 & 3.1 & 4.7 & 1.4 & 1.1 & $.5 *$ \\
\hline 71 & 11071 & 1 & 1 & -3.4 & 13.0 & -7.6 & 2.4 & 4.6 & 1.5 & .8 & $.4 *$ \\
\hline 71 & 11071 & 1 & 2 & 21.0 & 9.0 & -3.6 & 2.1 & 3.4 & 1.0 & .5 & $.4 *$ \\
\hline 71 & 11071 & 2 & 1 & -10.9 & 12.0 & 12.0 & .5 & 2.1 & 1.6 & -.6 & $.5 *$ \\
\hline 71 & 11071 & 6 & 1 & -6.1 & 8.1 & 7.6 & 1.1 & 1.4 & .9 & -.1 & $.4 *$ \\
\hline 71 & 11071 & 7 & 1 & 8.7 & 10.0 & 14.0 & 1.2 & .4 & 1.1 & -.5 & $.5 *$ \\
\hline 71 & 11071 & 7 & 2 & 27.0 & 12.0 & 1.5 & 1.6 & 3.8 & 1.3 & .2 & $.3 *$ \\
\hline 71 & 11071 & 7 & 3 & -9.9 & 9.1 & 2.0 & 1.9 & .1 & .8 & .2 & $.4 *$ \\
\hline 71 & 11071 & B & 1 & 16.0 & 13.0 & -7.6 & 2.5 & 5.0 & 1.5 & .8 & $.4 *$ \\
\hline 71 & 11071 & 8 & 2 & 10.0 & 7.8 & -4.3 & 2.0 & 1.9 & .9 & .3 & $.4 *$ \\
\hline 71 & 11071 & 8 & 3 & 3.5 & 10.0 &.$- t$ & 2.2 & 3.1 & 1.5 & .4 & $.5 *$ \\
\hline 73 & 11498 & 1 & 1 & 26.0 & 10.0 & -4.6 & 2.2 & 2.2 & .9 & .6 & $.4 *$ \\
\hline 73 & 11498 & 1 & 2 & 8.8 & 13.0 & -5.1 & 2.1 & 4.6 & 1.5 & .5 & $.5 *$ \\
\hline 73 & 11498 & 2 & 1 & 37.0 & 12.0 & 4.7 & 1.6 & 7.4 & 1.3 & -.0 & $.5 *$ \\
\hline 73 & 11498 & 4 & 1 & 170.0 & 23.0 & -13.9 & 3.7 & 12.0 & 2.3 & 1.9 & $.7 *$ \\
\hline 75 & 11829 & 1 & 1 & 46.0 & 11.0 & 1.0 &.$\dagger$ & 5.1 & 1.3 & .5 & $.9 *$ \\
\hline 75 & 11829 & 1 & 2 & 20.0 & 11.0 & 1.2 & .1 & 4.8 & 1.3 & .4 & $1.0 *$ \\
\hline 75 & 11829 & 2 & 1 & 21.0 & 10.0 & .8 & .1 & 3.4 & 1.0 & -1.2 & $1.0 *$ \\
\hline 75 & 11829 & 4 & 1 & 35.0 & 10.0 & 1.0 & .1 & 5.9 & 1.3 & .4 & $.9 * k$ \\
\hline 77 & 12107 & 1 & 1 & 25.0 & 11.0 & 10.0 & 1.6 & 3.7 & 1.1 & -.1 & $.5 *$ \\
\hline 77 & 12107 & 1 & 2 & 3.5 & 12.0 & -9.6 & 2.5 & 1.0 & 1.3 & 1.0 & $.6 *$ \\
\hline 77 & 12107 & 2 & 1 & 12.3 & 9.6 & 1.7 & 1.7 & 3.7 & 1.0 & .2 & $.4 *$ \\
\hline 77 & 12107 & 5 & $i$ & 17.7 & 12.5 & -5.3 & 2.0 & .2 & 1.4 & .5 & $.5 *$ \\
\hline 79 & 12385 & 1 & 1 & 23.5 & 10.4 & .6 & 2.0 & 2.3 & 1.0 & .2 & $.4 *$ \\
\hline 79 & 12305 & 1 & 2 & 15.9 & 13.4 & -20.5 & 2.9 & 1.3 & 1.4 & 1.7 & $.6 *$ \\
\hline 79 & 12385 & 2 & 1 & 19.5 & 12.7 & 2.8 & 1.8 & 3.8 & 1.4 & .1 & $.5 *$ \\
\hline 79 & 12385 & 2 & 2 & 70.6 & 11.4 & -1.1 & 2.1 & B. 0 & 1.4 & .4 & $.5 * k$ \\
\hline 79 & 12385 & 4 & 1 & 127.0 & 20.0 & & & 9.2 & 2.1 & 2.9 & $1.1 \%$ \\
\hline
\end{tabular}




\begin{tabular}{|c|c|c|c|c|c|c|c|c|c|c|c|}
\hline STAKE & DISTANCE & SAMF & & THL & & & & & & UR & \\
\hline No. & HETERS & TYPE & REP. & LEVEL & ERROR & LEVEL & ERROR & LEVEL & ERROR & LEVEL & ERROR \\
\hline 81 & 12728 & 1 & 1 & 8.8 & 13.1 & -11.4 & 2.9 & 3.5 & 1.5 & 1.1 & $.4 *$ \\
\hline 81 & 12728 & 1 & 2 & 3.7 & 9.2 & -1.9 & $1 . \theta$ & 1.8 & .9 & .2 & $.4 *$ \\
\hline 81 & 12728 & 1 & 3 & 23.0 & 13.1 & 6.3 & 1.2 & .4 & 1.2 & -.1 & $.4 *$ \\
\hline 81 & 12728 & 4 & 1 & 96.0 & 20.0 & & & 6.1 & 2.1 & .8 & $1.1 \%$ \\
\hline 81 & 12728 & 6 & 1 & -12.9 & 8.5 & & & 1.1 & .9 & .9 & $.9 *$ \\
\hline 81 & 12728 & 7 & 1 & 19.8 & 9.3 & -7.7 & 2.5 & 3.5 & .9 & .8 & $.4 *$ \\
\hline 81 & 12728 & 7 & 2 & 62.0 & 15.5 & -16.3 & 3.0 & 5.2 & 1.6 & 1.4 & $.5 *$ \\
\hline 81 & 12728 & 7 & 3 & 11.0 & 8.7 & -8.2 & 2.5 & .6 & 1.4 & .8 & $.4 *$ \\
\hline 81 & 12728 & 8 & 1 & 19.8 & 10.4 & -4.2 & 2.2 & 4.0 & 1.0 & .5 & $.4 *$ \\
\hline 81 & 12728 & B & 2 & B.9 & 11.6 & 4.5 & 1.3 & 1.5 & 1.4 & -.1 & $.4 *$ \\
\hline 81 & $1272 B$ & 8 & 3 & -3.6 & 7.9 & -7.1 & 2.5 & .4 & .9 & .7 & $.4 *$ \\
\hline 83 & 13021 & 1 & 1 & 0.7 & 9.2 & .3 & 1.9 & 2.3 & .9 & .2 & $.4 *$ \\
\hline 83 & 13021 & 2 & 1 & 23.0 & 12,0 & 5.9 & 1.3 & 2.7 & 1.4 & -.0 & $.5 *$ \\
\hline 83 & 13021 & 4 & $i$ & 58.0 & 12.0 & .8 & .2 & 6.5 & 1.2 & 1.1 & .90 \\
\hline 85 & 13362 & 1 & 1 & .0 & 12.0 & 3.2 & 1.6 & .2 & 1.6 & 1.2 & .90 \\
\hline 85 & 13362 & 2 & 1 & 19.0 & 11.0 & 10.0 & 2.4 & 2.8 & 1.1 & .7 & $1.0 *$ \\
\hline 85 & 13362 & 4 & 1 & 69.0 & 18.0 & 4.3 & 2.0 & 11.1 & 2.1 & 2.3 & $1.3 *$ \\
\hline B7 & 13643 & 1 & 1 & 23.0 & 11.6 & -3.1 & 2.2 & 2.2 & 1.4 & .5 & $.4 *$ \\
\hline 87 & 13643 & 2 & 1 & 8.7 & 8.8 & -3.0 & 1.9 & 3.4 & 1.1 & .4 & .40 \\
\hline 87 & 13643 & 3 & $\uparrow$ & B5. 0 & 18.0 & .9 & .2 & 4.2 & 2.1 & .0 & $1.1 *$ \\
\hline 87 & 13643 & 4 & 1 & 33.0 & 12.0 & 5.0 & 2.0 & 4.1 & 1.2 & .0 & $.9:$ \\
\hline 97 & 13643 & 4 & 2 & 32.1 & 12.0 & -.0 & 2.4 & 3.0 & 1.2 & .5 & $.4 *$ \\
\hline 89 & 13967 & 1 & 1 & 47.0 & 10.0 & -5.4 & 2.3 & 4.0 & 1.1 & .6 & $.4 *$ \\
\hline B9 & 13967 & 4 & 1 & 35.4 & 14.3 & 9.5 & 1.4 & 4.6 & 1.6 & -.1 & $.6 *$ \\
\hline 89 & 13967 & 9 & 1 & 14.0 & 8.8 & -18.9 & 2.9 & 2.2 & 1.1 & 1.4 & $.5 *$ \\
\hline 91 & 14238 & 1 & 1 & 14.9 & 10.2 & -.8 & 2.2 & 3.9 & 1.1 & .4 & $.5 \%$ \\
\hline 91 & 14238 & 2 & 1 & 90.3 & 16.3 & -2.0 & 2.7 & 7.7 & 1.8 & .7 & $.5 *$ \\
\hline 91 & 14238 & 6 & 1 & -16.9 & 9.8 & 1.9 & .2 & .6 & 1.1 & -.4 & $1.2 *$ \\
\hline 91 & 14238 & 7 & 1 & 3.7 & 7.7 & -5.8 & 2.1 & .4 & 1.0 & .5 & $.3 *$ \\
\hline 91 & 14238 & 7 & 2 & 8.8 & 12.4 & 7.3 & .9 & 2.5 & 1.4 & -.2 & $.4 *$ \\
\hline 91 & 14238 & 7 & 3 & 6.2 & 7.5 & 4.7 & 1.1 & 1.5 & .9 & -.0 & $.4 *$ \\
\hline 91 & 14238 & 8 & 1 & 26.6 & 14.7 & 5.1 & 1.3 & 1.7 & 1.5 & -.1 & $.5 t$ \\
\hline 91 & 14238 & 8 & 2 & .0 & 9.0 & .9 & 1.8 & 2.5 & 1.2 & .2 & .40 \\
\hline 91 & 14238 & 8 & 3 & -6.9 & 10.0 & 8.0 & .7 & 2.5 & 1.2 & -.3 & .40 \\
\hline 93 & 14586 & 1 & 1 & 40.8 & 14.6 & -11.8 & 2.8 & 6.6 & 1.3 & 1.1 & .5 \\
\hline 93 & 14586 & 1 & 2 & 7.4 & 10.0 & -9.2 & 2.6 & 4.7 & 1.1 & .9 & $.4 *$ \\
\hline 93 & 14586 & 2 & 1 & 88.6 & 16.8 & -8.0 & 2.7 & 7.1 & 1.8 & 1.1 & .61 \\
\hline 95 & 14874 & 1 & 1 & 14.0 & 8.5 & 6.3 & 1.6 & 2.6 & 1.0 & .4 & $.9 *$ \\
\hline 95 & 14874 & 2 & 1 & 16.0 & 11.0 & & & 2.9 & 1.1 & -.1 & .84 \\
\hline 95 & 14874 & 4 & $i$ & 8.9 & 15.0 & 7.6 & .9 & .8 & 1.7 & .0 & $1.2 *$ \\
\hline 95 & 14874 & 4 & 2 & 139.0 & 17.0 & 12.0 & 2.3 & 6.2 & 1.5 & .9 & $1.1 *$ \\
\hline
\end{tabular}




\begin{tabular}{|c|c|c|c|c|c|c|c|c|c|c|c|}
\hline STAKE & DISTANCE & \multicolumn{2}{|c|}{ SAHPLE } & \multicolumn{2}{|c|}{ THORIUH } & \multicolumn{2}{|c|}{ RAUIUH } & \multicolumn{2}{|c|}{ LEAU } & \multicolumn{2}{|c|}{ URANIUH } \\
\hline No. & HETERS & TYPE & REP. & LEVEL & ERROR & LEVEL & ERROR & LEVEL & ERROR & LEVEL & ERROR \\
\hline 97 & 15150 & 1 & 1 & 11.1 & B.7 & 8.2 & 1.1 & 2.3 & .9 & -.2 & $.4 *$ \\
\hline 97 & 15150 & 1 & 2 & 49.6 & 15.0 & 1.3 & 1.7 & 3.3 & 1.8 & .1 & $.5 *$ \\
\hline 99 & 15448 & 1 & 1 & 34.0 & 12.0 & 2.8 & 2.0 & 4.0 & 1.5 & .3 & $.6 *$ \\
\hline 99 & 15448 & 1 & 2 & 36.0 & 11.0 & -9.2 & 2.6 & 2.1 & 1.1 & .9 & $.5 *$ \\
\hline 99 & 15448 & 4 & 1 & 38.0 & 11.0 & 0.0 & 2.0 & 4.8 & 1.3 & .5 & $1.0 *$ \\
\hline 99 & 15448 & 4 & 2 & 110.0 & 19.0 & & & .0 & 2.1 & 1.0 & $1.1 \%$ \\
\hline 99 & 15448 & 4 & 3 & 27.0 & 12.0 & 1.2 & .1 & 4.4 & 1.3 & -1.2 & $1.0 *$ \\
\hline 99 & 15448 & 4 & 4 & 79.0 & 15.0 & 2.5 & .3 & 15.0 & $1 . \theta$ & .7 & $1.3 *$ \\
\hline 101 & 15712 & 1 & 1 & 2.5 & 7.4 & -.2 & 1.7 & 3.6 & 1.0 & .3 & $.4 *$ \\
\hline 101 & 15712 & 2 & 1 & 28.0 & 12.0 & -6.7 & 2.5 & 3.1 & 1.4 & .8 & $.5 *$ \\
\hline 101 & 15712 & 4 & 1 & 35.0 & 14.0 & 1.8 & .2 & 3.1 & 1.9 & -.3 & 1.24 \\
\hline 101 & 15712 & 6 & 1 & -1.7 & 11.0 & -4.2 & 2.2 & .4 & 1.1 & .5 & $.5 *$ \\
\hline 101 & 15712 & 7 & 1 & 10.0 & 8.6 & .4 & 1.7 & .1 & .8 & .2 & $.3 *$ \\
\hline 101 & 15712 & 7 & 2 & 7.1 & 11.0 & 4.0 & 1.5 & 2.3 & 1.3 & .0 & $.4 \neq$ \\
\hline 101 & 15712 & 7 & 3 & 2.5 & 8.0 & 8.4 & 1.0 & 1.4 & 1.0 &.$- t$ & $.4 *$ \\
\hline 101 & 15712 & 8 & 1 & 5.3 & 12.0 & -7.9 & 2.3 & 2.3 & 1.2 & .7 & $.3 *$ \\
\hline 101 & 15712 & 8 & 2 & 9.7 & 7.5 & 2.1 & 1.5 & .6 & .9 & .1 & $.4 *$ \\
\hline 101 & 15712 & 8 & 3 & -22.9 & 12.0 & 7.3 & 1.6 & .6 & 1.3 & -.0 & $.4 *$ \\
\hline 103 & 16062 & 1 & 1 & 38.0 & 11.0 & -1.4 & 2.2 & 6.8 & 1.3 & .5 & $.5 \%$ \\
\hline 103 & 16062 & 2 & 1 & 51.0 & 15.0 & -6.7 & 2.7 & 6.1 & 1.7 & 1.0 & $.5 *$ \\
\hline 103 & 16062 & 4 & 1 & 20.0 & 10.0 & 2.9 & 1.8 & 4.5 & 1.0 & .2 & $.3 *$ \\
\hline 105 & 16264 & 1 & 1 & 18.0 & 12.0 & & & 5.0 & 1.5 & -.7 & $.8 *$ \\
\hline 105 & 16264 & 2 & 1 & 9.9 & 9.6 & 1.0 & .1 & .7 & 1.1 & 2.3 & $1.1 *$ \\
\hline 105 & 16264 & 9 & 1 & 210.0 & 20.0 & 2.9 & .3 & B. 8 & 1.6 & .4 & $1.1 *$ \\
\hline 107 & 16699 & 1 & 1 & 12.0 & 13.0 & -5.2 & 2.0 & .2 & 1.3 & .6 & $.5 *$ \\
\hline 107 & 16689 & 2 & 1 & 36,0 & 11.0 & -.7 & .7 & 1.6 & 1.0 & .2 & $.5 *$ \\
\hline 107 & 16689 & 2 & 2 & -2.4 & 6.8 & 2.0 & 1.3 & 1.8 & .9 & -.0 & $.3 *$ \\
\hline 107 & 16689 & 4 & 1 & 37.0 & 10.0 & -7.3 & 2.3 & 2.3 & 1.1 & .6 & $.4 *$ \\
\hline 109 & 17015 & 1 & 1 & 16.0 & 13.0 & 6.4 & 1.1 & 3.1 & 1.4 & -.2 & .44 \\
\hline 109 & 17015 & 2 & 1 & 70.8 & 14.8 & -4.3 & 2.1 & 2.3 & 1.3 & .6 & $.5 *$ \\
\hline 111 & 17293 & 1 & 1 & 39.0 & 13.0 & -1.7 & 2.0 & 2.3 & 9.6 & .3 & $.5 *$ \\
\hline 111 & 17293 & 2 & 1 & 40.0 & 11.0 & -6.0 & 2.7 & 7.7 & 1.2 & 1.0 & $.4 *$ \\
\hline 111 & 17293 & 7 & 1 & 48.0 & 13.0 & -.2 & .4 & 2.1 & 1.2 & -2.7 & $.4 *$ \\
\hline 111 & 17293 & 7 & 2 & 3.5 & 10.0 & 4.8 & 1.1 & 1.0 & 1.6 & -.1 & $.4 *$ \\
\hline 111 & 17293 & 7 & 3 & 7.4 & 7.0 & -.0 & 1.6 & .8 & .8 & .2 & $.4 *$ \\
\hline 111 & 17293 & B & 1 & 8.7 & 7.5 & 6.3 & .7 & 2.5 & 1.0 & -.2 & $.3 *$ \\
\hline 111 & 17293 & 8 & 2 & -2.4 & 6.8 & 5.1 & $t .3$ & 1.0 & .9 & -.1 & $.4 *$ \\
\hline 111 & 17293 & 8 & 3 & -17.9 & 12.0 & -1.8 & 2.0 & .2 & 1.3 & .4 & $.3 *$ \\
\hline 113 & 17663 & 1 & 1 & 17.7 & 13.7 & .9 & 1.8 & 6.3 & 1.6 & .2 & $.5 *$ \\
\hline 113 & 17663 & 2 & 1 & 50.8 & 11.0 & -2.3 & 2.2 & 6.5 & 1.3 & .5 & $.5 *$ \\
\hline 115 & 17965 & 1 & 1 & 38.0 & 10.0 & & & 4.1 & 1.1 & 1.1 & $1.0 *$ \\
\hline
\end{tabular}




\begin{tabular}{|c|c|c|c|c|c|c|c|c|c|c|c|}
\hline STAKE & DISTANCE & \multicolumn{2}{|c|}{ SAMPLE } & \multicolumn{2}{|c|}{ THORIUn } & \multicolumn{2}{|c|}{ RAIIUK } & \multicolumn{2}{|c|}{ LEAU } & \multicolumn{2}{|c|}{ URANIUH } \\
\hline NO. & METERS & TYFE & REP. & LEVEL & ERROR & LEVEL & ERROK & LEVEL & ERROR & LEVEL & ERROR \\
\hline 115 & 17965 & 2 & $t$ & 30.0 & 9.3 &.$B$ & .2 & -.3 & 1.0 & -.1 & .94 \\
\hline 117 & 18277 & 1 & 1 & 23.5 & 9.0 & -2.2 & 2.1 & 1.4 & 1.0 & .4 & $.4 *$ \\
\hline 117 & 18277 & 2 & 1 & 67.3 & 13.7 & -18.5 & 3.0 & 4.2 & 1.6 & 1.7 & $.5 *$ \\
\hline 119 & 18630 & 1 & $t$ & 45.8 & 10.3 & 5.1 & 1.4 & 2.3 & 1.2 & -.0 & $.3 *$ \\
\hline 119 & 18630 & 2 & 1 & 26.6 & 14.3 & 4.7 & 1.7 & 2.3 & 1.8 & -.0 & $.5 *$ \\
\hline 121 & 18887 & 1 & 1 & -8.7 & 12.0 & 5.6 & 1.4 & 1.0 & 1.4 & $=.0$ & $.5 *$ \\
\hline 121 & 18887 & 2 & 1 & 42.1 & 11.5 & -1.2 & 2.2 & 3.2 & 1.1 & .5 & $.4 *$ \\
\hline 121 & 18887 & 6 & 1 & -9.8 & 9.1 & 2.0 & 1.7 & 1.0 & .9 & .2 & $.4 *$ \\
\hline 121 & 18887 & 7 & $\uparrow$ & 6.2 & 7.9 & -3.1 & 2.1 & 2.2 & 1.0 & .5 & $.4 *$ \\
\hline 121 & 18887 & 7 & 2 & -17.6 & 10.9 & -1.5 & 2.7 & 3.7 & 1.4 & .4 & $.5 *$ \\
\hline 121 & 18887 & 7 & 3 & -15.8 & 10.8 & 2.9 & 1.8 & 1.2 & 1.4 & .2 & $.4 *$ \\
\hline 121 & 18887 & 8 & 1 & 21.0 & 9.7 & -8.5 & 2.3 & 3.2 & .9 & .8 & $.4 *$ \\
\hline 121 & 18887 & 8 & 2 & 7.1 & 12.3 & 19.7 & .7 & .2 & 1.2 & -.5 & $.4 *$ \\
\hline 121 & 18887 & B & 3 & 14.9 & 7.2 & 3.1 & 1.2 & 1.5 & .8 & -.1 & $.3 *$ \\
\hline 123 & 19209 & 1 & 1 & 54.9 & 13.1 & -1.1 & 2.2 & 4.2 & 1.4 & .5 & $.5 *$ \\
\hline 123 & 19209 & 2 & 1 & 34.6 & 9.6 & 2.2 & 2.0 & 1.0 & 1.0 & .2 & $.4 *$ \\
\hline 123 & 19209 & 2 & 2 & 43.3 & 11.8 & 7.6 & 1.2 & 7.2 & 1.2 & -.1 & $.4 *$ \\
\hline 125 & 19555 & 1 & 1 & 10.0 & 11.0 & 2.6 & 1.4 & .8 & 1.4 & -2.2 & $1.0 \%$ \\
\hline 125 & 19555 & 2 & 1. & 71.0 & 13.0 & 7.7 & 1.8 & 4.0 & 1.2 & -.6 & $1.1 *$ \\
\hline 127 & 19879 & 1 & 1 & 100.9 & 18.3 & -9.5 & 3.4 & 4.3 & 1.8 & 1.7 & $.6: k$ \\
\hline 127 & 19879 & 1 & 2 & 12.3 & 11.9 & 4.9 & 1.8 & 1.0 & 1.4 & .0 & $.5 *$ \\
\hline 127 & 19879 & 2 & 1 & 82.9 & 12.8 & -.4 & 2.5 & 6.6 & 1.3 & .5 & $.5 *$ \\
\hline 127 & 19879 & 2 & 2 & 84.2 & 14.7 & -5.5 & 2.6 & 7.6 & 1.4 & .8 & $.5 *$ \\
\hline 129 & 20189 & 1 & 1 & 28.3 & 12.3 & -2.5 & 2.1 & 1.7 & 1.5 & .5 & $.5 *$ \\
\hline 129 & 20189 & 2 & 1 & 65.0 & 17.0 & 1.3 & .2 & 4.0 & 1.8 & -.7 & 1.24 \\
\hline 129 & 20189 & 2 & 2 & 37.0 & 13.0 & 1.0 & .1 & 1.9 & 1.7 & .8 & $.9 *$ \\
\hline 129 & 20189 & 4 & 1 & 27.2 & 11.2 & -8.7 & 2.4 & 3.9 & 1.2 & .8 & $.4 *$ \\
\hline 131 & 20427 & 1 & 1 & 48.0 & 13.0 & .4 & .1 & 4.0 & 1.6 & .6 & $1.0 \%$ \\
\hline 131 & 20427 & 2 & 1 & 30.0 & 15.0 & 1.0 & .1 & 3.8 & 1.6 & .6 & $1.0 *$ \\
\hline 131 & 20427 & 4 & 1 & 58.0 & 13.0 & .7 & .1 & 2.2 & 1.2 & .0 & $1.0 *$ \\
\hline 131 & 20427 & 4 & 2 & 44.0 & 14.0 & 1.5 & .2 & 4.2 & 1.8 & .0 & $1.1 *$ \\
\hline 131 & 20427 & 4 & 3 & 120.0 & 16.0 & B. 5 & 2.1 & 9.1 & 1.6 & 11.0 & 1.5 \\
\hline 131 & 20427 & 6 & 1 & -1.7 & 9.2 & 1.1 & 1.5 & 2.5 & 1.1 & 1.8 & 1.0 \\
\hline 131 & 20427 & 6 & 2 & .0 & 12.0 & 1.4 & .2 & 4.2 & 1.7 & -3.8 & $1.1 *$ \\
\hline 131 & 20427 & 8 & 1 & 23.0 & 8.7 & 1.4 & 1.4 & 2.3 & .8 & 4.8 & 1.0 \\
\hline 131 & 20427 & 8 & 2 & 44.0 & 12.0 & 2.6 & 1.4 & 1.8 & 1.3 & 5.1 & 1.3 \\
\hline 131 & 20427 & B & 3 & 7.4 & 8.7 & 6.0 & 1.7 & 1.9 & 1.0 & 2.1 & 1.1 \\
\hline 133 & 20789 & 1 & 1 & 8.9 & 10.0 & 4.0 & 1.6 & 1.9 & 1.2 & 3.0 & 1.2 \\
\hline 133 & 20789 & 1 & 2 & 30.0 & 10.0 & 3.7 & 1.6 & 2.1 & 1.3 & 3.6 & 1.2 \\
\hline 133 & 20789 & 2 & 1 & 7.4 & B. 0 & 1.6 & 1.3 & 1.5 & .9 & 4.8 & 1.1 \\
\hline 133 & 20789 & 2 & 2 & 100.0 & 16.0 & 16.0 & 1.7 & 5.4 & 1.4 & 1.3 & $1.0 *$ \\
\hline
\end{tabular}




\begin{tabular}{|c|c|c|c|c|c|c|c|c|c|c|c|c|}
\hline & STAKE & DISTANCE & SAH & LE & THO & & & & & & UR & \\
\hline & NO. & HETERS & TYPE & REP. & LEVEL & ERROR & LEVEL & ERROR & LEVEL & ERROR & LEVEL & ERROR \\
\hline & 135 & 21077 & 1 & 1 & 37.0 & 14.0 & 3.2 & 1.6 & 1.0 & 1.7 & .8 & $.9 *$ \\
\hline & 135 & 21077 & 1 & 2 & 67.0 & 18.0 & & & 4.0 & 1.7 & .6 & $1.1 *$ \\
\hline & 137 & 21358 & 1 & 1 & 22.0 & 8.0 & 4.1 & 1.4 & 2.2 & .9 & 2.5 & .9 \\
\hline & 137 & 21635 & 2 & 1 & 55.0 & 16.0 & .6 & 1.6 & 2.0 & 1.5 & 2.8 & 1.4 \\
\hline & 139 & 21644 & 1 & 1 & 6.2 & 9.6 & 5.5 & 1.9 & 2.5 & 1.0 & 3.6 & 1.2 \\
\hline & 139 & 21644 & 2 & 1 & 35.0 & 11.0 & 1.4 & 1.7 & 1.7 & 1.5 & 4.1 & 1.2 \\
\hline & 141 & 21942 & 1 & 1 & 55.0 & 12.0 & 1.4 & 1.5 & 5.4 & 1.2 & 6.7 & 1.2 \\
\hline & 141 & 21942 & 1 & 2 & 12.0 & 13.0 & $1 . \theta$ & 1.7 & 4.0 & 1.3 & 3.0 & 1.3 \\
\hline & 141 & 21942 & 6 & 1 & 11.0 & 9.2 & & & 1.1 & 1.1 & 1.1 & $1.0 *$ \\
\hline & 141 & 21942 & 6 & 2 & -10.9 & 13.0 & 5.5 & 1.8 & 3.0 & 1.4 & 3.7 & 1.5 \\
\hline & 141 & 21942 & 6 & 3 & 16.0 & 10.0 & 1.1 & 1.3 & 1.9 & 1.3 & 2.1 & 1.2 \\
\hline & 141 & 21942 & 7 & 1 & -2.4 & 7.8 & 1.1 & 1.5 & 1.4 & .9 & 5.7 & 1.0 \\
\hline & 141 & 21942 & 7 & 2 & -10.9 & 13.0 & 6.1 & 1.9 & 2.5 & 1.3 & 2.8 & 1.5 \\
\hline & 141 & 21942 & 7 & 3 & 6.2 & 8.5 & 3.6 & 1.6 & .6 & 1.0 & 3.1 & 1.1 \\
\hline & 141 & 21942 & $\theta$ & 1 & 14.0 & 11.0 & 4.9 & 1.7 & 6.2 & 1.2 & 6.3 & 1.2 \\
\hline & 141 & 21942 & 8 & 2 & 30.0 & 12.0 & 4.3 & 1.7 & 2.5 & 1.3 & 3.8 & 1.1 \\
\hline & $\$ 41$ & 21942 & 8 & 3 & -6.1 & 8.1 & 1.4 & 1.2 & .8 & .8 & 2.7 & .9 \\
\hline & 143 & 22295 & 1 & $i$ & 66.0 & 12.0 & 2.5 & 1.6 & 1.6 & 1.2 & 6.7 & 1.3 \\
\hline & 143 & 22295 & 1 & 2 & 35.0 & 15.0 & 5.5 & 1.6 & 1.5 & 1.4 & 2.7 & 1.3 \\
\hline i & 143 & 22295 & 2 & 1 & 18.0 & 11.0 & 8.6 & 1.8 & 3.3 & 1.2 & 4.8 & 1.1 \\
\hline 0 & 145 & 22583 & 1 & 1 & 44.0 & 14.0 & & & 5.0 & 1.7 & .6 & $.9 *$ \\
\hline & 145 & 22583 & 1 & 2 & 80.0 & 16.0 & 5.5 & 1.8 & 2.9 & 1.8 & .4 & $1.2 *$ \\
\hline & 145 & 22583 & 2 & 2 & 25.0 & 14.0 & 2.0 & 1.5 & 2.7 & 1.7 & -.5 & $.8 *$ \\
\hline & 145 & 22583 & 2 & 3 & 31.0 & 10.0 & 3.8 & 1.5 & 3.4 & 1.0 & -.4 & $1.0 *$ \\
\hline & 147 & 22912 & 1 & 1 & 55.0 & 15.0 & .8 & 1.9 & 2.7 & 1.5 & 5.3 & 1.4 \\
\hline & 147 & 22912 & 1 & 2 & 83.0 & 13.0 & .8 & 1.7 & 5.0 & 1.3 & 7.1 & 1.4 \\
\hline & 147 & 22912 & 2 & 1 & 112.0 & 20.0 & 10.0 & 2.4 & 9.6 & 2.4 & 2.0 & $1.3 *$ \\
\hline & 149 & 23186 & 1 & 1 & 25.0 & 12.0 & -.2 & 1.9 & 5.4 & 1.4 & 3.9 & 1.3 \\
\hline & 149 & 23186 & 1 & 2 & 90.0 & 15.0 & 4.8 & 1.7 & 5.8 & 1.3 & 7,9 & 1.4 \\
\hline & 149 & 23186 & 2 & 1 & 18.0 & 14.0 & 2.4 & 1.9 & 3.1 & 1.6 & 2.7 & 4.3 \\
\hline & 151 & 23484 & 1 & 1 & 2.5 & 8.7 & 3.3 & 1.7 & 1.6 & .8 & 5.9 & 1.2 \\
\hline & 151 & 23484 & 1 & 2 & 60.0 & 16.0 & -2.2 & 2.3 & 2.3 & 1.6 & 0.5 & 1.7 \\
\hline & 151 & 23484 & 2 & 1 & -2.4 & 9.1 & 4.3 & 1.7 & 4.0 & 1.0 & 4.3 & 1.0 \\
\hline & 151 & 23484 & 4 & 1 & 147.0 & 23.0 & -6.3 & 3.0 & 20.0 & 2.8 & 18.0 & 2.3 \\
\hline & 151 & 23184 & 6 & 1 & 8.9 & 9.8 & 1.0 & 1.4 & .2 & 1.3 & 1.6 & 1.1 \\
\hline & 151 & 23484 & 6 & 2 & -1.1 & 7.3 & 2.4 & 1.3 & .8 & .9 & 3.6 & .9 \\
\hline & 151 & 23484 & 7 & 1 & -11.9 & 6.8 & 1.2 & 1.3 & .1 & .6 & 1.4 & .9 \\
\hline & 151 & 23484 & 7 & 2 & -3.4 & 9.0 & 1.2 & 1.5 & .2 & 1.0 & 2.0 & 1.0 \\
\hline & 151 & 23484 & 7 & 3 & -3.6 & 6.9 & 3.8 & 1.6 & .6 &.$\theta$ & 3.2 & .9 \\
\hline & 151 & 23484 & 7 & 4 & 8.9 & 12.0 & 1.7 & 2.0 & 1.9 & 1.2 & 4.3 & 1.3 \\
\hline & 151 & 23484 & 7 & 5 & -7.3 & 7.4 & 2.3 & 1.4 & .6 & .8 & 1.8 & 1.0 \\
\hline
\end{tabular}




\begin{tabular}{|c|c|c|c|c|c|c|c|c|c|c|c|}
\hline STAKE & DISTANCE & SAH & PLE & THO & & & & & & UJRF & \\
\hline No. & METERS & TYPE & KEP. & LEVEL & ERROR & LEVEL & ERROR & LEVEL & EKROR & LEUEL & ERKOK \\
\hline 151 & 23484 & 7 & 6 & 5.3 & 11.0 & .8 & 1.6 & .0 & 1.2 & 2.9 & 1.1 \\
\hline 153 & $2385 b$ & 1 & 1 & 23.0 & 10.0 & 2.0 & 1.6 & 3.4 & 1.1 & 2.0 & 1.1 \\
\hline 153 & 23856 & 1 & 2 & 12.0 & 11.0 & 2.8 & 1.7 & .7 & .9 & 5.3 & 1.1 \\
\hline 153 & 23856 & 2 & 1 & -8.8 & 12.0 & -2.5 & 1.8 & 2.7 & 1.3 & 4.3 & 1.3 \\
\hline 153 & 23856 & 2 & 2 & 30.0 & 13.0 & -.3 & 1.9 & 4.0 & 1.4 & 2.9 & 1.3 \\
\hline 155 & 24209 & 1 & 1 & 77.0 & 13.0 & 6.6 & 1.9 & 3.4 & 1.2 & -.8 & $1.1 *$ \\
\hline 155 & 24209 & 1 & 2 & 4.9 & 8.9 & 5.6 & 1.8 & .4 & 1.0 & 4.5 & 1.1 \\
\hline 155 & 24209 & 2 & 1 & 42.0 & 12.0 & 1.9 & 1.5 & 1.4 & 1.2 & -.3 & $1.0 *$ \\
\hline 157 & 24569 & 1 & 1 & 53.1 & 14.2 & 7.5 & 2.0 & 6.5 & 1.8 & .0 & $.5 *$ \\
\hline 157 & 24569 & 1 & 2 & 41.0 & 15.0 & 4,8 & 2.0 & 5.4 & 1.7 & .1 & $.6 *$ \\
\hline 157 & 24569 & 4 & 1 & 21.2 & 11.5 & 4.7 & 1.6 & 2.7 & 1.3 & -.0 & $.5 *$ \\
\hline 159 & 24857 & 1 & 1 & 23.0 & 14.0 & -13.9 & 2.7 & 2.7 & 1.6 & 1.2 & $.5 *$ \\
\hline 159 & 24857 & 1 & 2 & 43.0 & 10.0 & 6.8 & 1.1 & 3.2 & 1.1 & -.1 & $.4 *$ \\
\hline 159 & 24857 & 2 & 1 & 44.0 & 11.0 & -7.8 & 2.4 & 1.5 & 1.1 & .8 & $.4 *$ \\
\hline 159 & 24857 & 4 & 1 & 237.0 & 25.0 & & & 16.0 & 2.6 & 1.4 & $1.3 *$ \\
\hline 159 & 24857 & 5 & 1 & 16.0 & 14.0 & & & 2.7 & 1.7 & .4 & $1.0 *$ \\
\hline 159 & 24857 & 5 & 2 & 74.0 & 20.0 & & & 6.1 & 2.3 & .2 & $1.0 *$ \\
\hline 161 & 25162 & 1 & 1 & 55.0 & 16.0 & -1.6 & 2.4 & 4.0 & 1.7 & .5 & $.5 *$ \\
\hline 161 & 25162 & 1 & 2 & 25.0 & 12.0 & 1.3 & 1.6 & 2.3 & 1.4 & 1.0 & $.6 *$ \\
\hline 161 & 25162 & 1 & 3 & 35.0 & 11.0 & 1.0 & 2.0 & 5.1 & 1.1 & .2 & $.4 *$ \\
\hline 161 & 25162 & 1 & 4 & 27.0 & 12.0 & 2.7 & 1.7 & 2.5 & 1.1 & 3.0 & 1.2 \\
\hline 161 & 25162 & 4 & 1 & 152.0 & 19.0 & 9.0 & 2.3 & 8.1 & 1.7 & 11.0 & 1.6 \\
\hline 161 & 25162 & 5 & 1 & 74.0 & 17.0 & 3.7 & 1.6 & 5.2 & 1.7 & 6.8 & 1.6 \\
\hline 161 & 25162 & 6 & 1 & -8.7 & 9.5 & & & -.3 & 1.3 & 2.2 & $1.1 *$ \\
\hline 161 & 25162 & 6 & 2 & -8.6 & 7.5 & & & 2.6 & .8 & -.3 & . $8 *$ \\
\hline 161 & 25162 & 8 & 1 & 15.0 & 9.3 & 2.9 & 1.7 & .0 & .9 & .1 & $.4 *$ \\
\hline 161 & 25162 & 8 & 2 & 12.4 & 0.6 & 1.6 & 1.8 & 1.4 & .9 & .1 & $.4 *$ \\
\hline 161 & 25162 & 8 & 3 & 5.3 & 11.0 & -6.3 & 2.4 & 1.2 & 1.2 & .7 & $.4 *$ \\
\hline 161 & 25162 & 9 & 1 & 226.8 & 25.4 & -13.6 & 4.0 & 22,3 & 2.9 & 2.1 & $.7 *$ \\
\hline 163 & 25445 & 2 & 1 & 22.0 & 10.0 & -1.7 & 2.1 & 1.9 & .9 & .5 & .44 \\
\hline 163 & 25445 & 2 & 2 & 32.0 & 13.0 & 1.1 & .1 & 1.7 & 1.7 & .2 & $1.0 *$ \\
\hline 163 & 25445 & 4 & 1 & 120.0 & 18.0 & 2.2 & .3 & 12.0 & 1.7 & -.1 & $1.0 *$ \\
\hline 165 & 25789 & 1 & 1 & 72.0 & 17.0 & 3.5 & 1.6 & 4.0 & 1.6 & 6.1 & 1.6 \\
\hline 167 & 26036 & 1 & 1 & .0 & 7.4 & 1.1 & 1.4 & 2.7 & 1.0 & 3.4 & 1.0 \\
\hline 167 & 26036 & 2 & 1 & 46.0 & 16.0 & 2.9 & 1.8 & 5.6 & 1.6 & 4.8 & 1.5 \\
\hline 169 & 26326 & 1 & 1 & 26.0 & 10.0 & 8.2 & 1.8 & 2.2 & 1.9 & 3.8 & 1.0 \\
\hline 169 & 26326 & 1 & 2 & .0 & 11.0 & .0 & 1.6 & .8 & 1.2 & 2.0 & 1.3 \\
\hline 169 & 26326 & 9 & 1 & 34.0 & 16.0 & 4.9 & 1.7 & 1.0 & 1.6 & -.3 & $1.0 \neq$ \\
\hline 171 & 26712 & 1 & 1 & 16.0 & 12.0 & 4.0 & 1.5 & 4.8 & 1.5 & 3.4 & 1.1 \\
\hline 171 & 26712 & 1 & 2 & 41.0 & 13.0 & 3.2 & 1.7 & 4.2 & 1.4 & 5.1 & 1.4 \\
\hline 171 & 26712 & 2 & 1 & 6.2 & 8.7 & 2.7 & 1.4 & 2.3 & 1.0 & 1.7 & 1.0 \\
\hline
\end{tabular}




\begin{tabular}{|c|c|c|c|c|c|c|c|c|c|c|c|}
\hline STAKE & DISTANCE & SAMP & LE & THO & & & & & & URA & \\
\hline NO. & AETERS & TYPE & REP. & LEVEL & ERROR & LEVEL & ERROR & LEVEL & ERROR & LEVEL & EKROK \\
\hline 171 & 26712 & 2 & 2 & 35.0 & 13.0 & 2.9 & 1.6 & .6 & 1.4 & 6.3 & 1.3 \\
\hline 171 & 26712 & 2 & 3 & 11.0 & 9.5 & 6.7 & 1.8 & -.3 & .9 & 2.1 & 1.1 \\
\hline 171 & 26712 & 6 & 1 & 25.0 & 13.0 & -.7 & 1.7 & 2.9 & 1.4 & 3.8 & 1.3 \\
\hline 171 & 26712 & 6 & 2 & 1.8 & 9.9 & -2.1 & 1.8 & 1.7 & 1.3 & 4.8 & 1.3 \\
\hline 171 & 26712 & 7 & 1 & 12.0 & 10.0 & 1.1 & 1.7 & 1.9 & 1.0 & 4.2 & 1.1 \\
\hline 171 & 26712 & 7 & 2 & 16.0 & 11.0 & 1.1 & 1.2 & .0 & .9 & 2.8 & 9.1 \\
\hline 171 & 26712 & 7 & 3 & 4.9 & 8.7 & 4.6 & 1.8 & .4 & .9 & 3.9 & 1.0 \\
\hline 171 & 26712 & 8 & 1 & 33.0 & 10.0 & 6.3 & 1.8 & 1.1 & 1.0 & 3.0 & 1.0 \\
\hline 171 & 26712 & B & 2 & -7.0 & 12.0 & .9 & 1.5 & 1.2 & 1.3 & 2.5 & 1.2 \\
\hline 171 & 26712 & 8 & 3 & -1.1 & B.3 & 2.4 & 1.5 & 1.5 & .7 & 1.4 & .8 \\
\hline 171 & 26712 & 9 & 1 & 25.0 & 11.0 & 1.2 & .4 & 4.6 & 1.5 & -.3 & $1.1 *$ \\
\hline 173 & 27029 & 1 & 1 & 53.0 & 11.0 & 4.6 & 1.7 & 3.4 & 1.2 & 6.6 & 1.2 \\
\hline 173 & 27029 & $i$ & 2 & 11.0 & 10.0 & -5.0 & 2.1 & 3.2 & 1.1 & .5 & $.5 *$ \\
\hline 173 & 27029 & 4 & 1 & 48.0 & 17.0 & 7.8 & 2.2 & 6.3 & 1.8 & .2 & $1.3 *$ \\
\hline 175 & 27315 & 1 & $i$ & 27.0 & 11.0 & 3.6 & 1.5 & 1.8 & 1.0 & 1.6 & $1.1 *$ \\
\hline 175 & 27315 & 1 & 2 & 32.0 & 11.0 & 3.6 & 1.7 & 2.5 & 1.1 & .9 & $.9: k$ \\
\hline 175 & 27315 & 2 & 1 & 105.0 & 17.0 & & & 8.3 & 1.5 & 2.0 & $1.0 *$ \\
\hline 175 & 27315 & 3 & 1 & 54.0 & 12.0 & 1.3 & .4 & 2.5 & 1.1 & -.1 & $1.0 *$ \\
\hline 175 & 27315 & 4 & 1 & 111.6 & 21.6 & .8 & 2.4 & 13.4 & 2.3 & .5 & $.7 *$ \\
\hline 175 & 27315 & 5 & 1 & 39.0 & 15.0 & 3.7 & .6 & 5.9 & 1.8 & 3.3 & $1.0 *$ \\
\hline 175 & 27315 & 6 & 1 & -6.1 & 8.7 & 1.2 & .2 & 1.0 & .9 & -.3 & $1.1 *$ \\
\hline 175 & 27315 & 6 & 3 & 3.7 & 8.7 & 1.5 & .2 & 2.8 & 1.1 & .5 & $1.1 *$ \\
\hline 177 & 27608 & 1 & 1 & -1.1 & 8.8 & 5.1 & 1.7 & .4 &.$B$ & 4.1 & 1.0 \\
\hline 177 & $2760 \mathrm{~B}$ & 2 & 1 & 21.0 & 13.0 & -.8 & 2.0 & .2 & 1.5 & 1.9 & 1.5 \\
\hline 177 & 27608 & 3 & 1 & 10.0 & 12.0 & 2.9 & .5 & 5.1 & 1.4 & -.6 & $1.0 \%$ \\
\hline 177 & 27608 & 4 & 1 & 60.0 & 16.0 & 2.0 & 2.1 & 5.4 & 1.6 & 5.3 & 1.5 \\
\hline 177 & 27600 & 9 & 1 & 40.0 & 10.0 & -2.0 & 2.0 & 2.0 & 1.1 & 5.8 & 1.2 \\
\hline 177 & 27608 & 9 & 2 & 73.0 & 14.0 & 3.6 & 1.8 & 2.9 & 1.3 & 6.1 & 1.3 \\
\hline 179 & 27944 & 1 & 1 & 18.0 & 13.0 & -2.9 & 2.1 & .4 & 1.4 & .6 & $.5 *$ \\
\hline 179 & 27944 & 1 & 2 & 48.0 & 12.0 & -1.7 & 2.1 & 3.3 & 1.7 & 7.6 & 1.3 \\
\hline 179 & 27944 & 2 & 1 & 38.0 & 12.0 & 3.4 & 1.8 & 5.0 & 1.2 & 5.2 & 1.1 \\
\hline 179 & 27944 & 3 & 1 & 35.0 & 18.0 & 3.2 & .5 & 8.1 & 2.0 & .8 & $1.2 *$ \\
\hline 101 & 28242 & 1 & 1 & 60.2 & 14.8 & -3.2 & 2.3 & 2.9 & 1.5 & .6 & $.4 *$ \\
\hline 181 & $2 \mathrm{~B} 242$ & 1 & 2 & 15.0 & 9.3 & 5.6 & 2.0 & 3.7 & 1.1 & 5.0 & 1.1 \\
\hline 181 & 28242 & 2 & 1 & 47.0 & 11.0 & & & 3.7 & 1.2 & .0 & $1.0 \%$ \\
\hline 181 & 28242 & 2 & 2 & 90.0 & 17.0 & .1 & 2.1 & 4.2 & 1.6 & 6.2 & 1.5 \\
\hline 181 & 28242 & 3 & 1 & 165.0 & 23.0 & 1.4 & 3.1 & 1.8 & 2.6 & 14.0 & 2.0 \\
\hline 181 & 28242 & 6 & 1 & -8.6 & 7.7 & 4.7 & 1.7 & 2.6 & 1.0 & 2.1 & 1.0 \\
\hline 181 & 28242 & 6 & 2 & -4.9 & 12.0 & & & 2.3 & 1.6 & .2 & $1.1 *$ \\
\hline 181 & 28242 & 6 & 3 & -6.1 & 7.5 & 2.6 & 1.5 & 2.7 & 1.1 & 3.0 & 1.0 \\
\hline 181 & 28242 & 7 & 1 & 30.0 & 12.0 & -4.8 & 1.8 & 2.7 & 1.4 & 6.0 & 1.2 \\
\hline
\end{tabular}




\begin{tabular}{|c|c|c|c|c|c|c|c|c|c|c|c|c|}
\hline & \multirow{3}{*}{$\begin{array}{l}\text { STAKE } \\
\text { NO. } \\
181\end{array}$} & \multirow{2}{*}{$\begin{array}{l}\text { IISTANCE } \\
\text { HETERS }\end{array}$} & \multicolumn{2}{|c|}{ SAAFLE } & \multicolumn{2}{|c|}{ THORIUH } & \multicolumn{2}{|c|}{ RADIUA } & \multicolumn{2}{|c|}{ LEAI } & \multicolumn{2}{|c|}{ URANIUH } \\
\hline & & & TYFE & REF. & LEVEL & ERROR & LEVEL & ERROR & LEVEL & ERROR & LEVEL & ERROR \\
\hline & & 28242 & 7 & 2 & -1.1 & $\theta .1$ & 3.6 & 1.5 & 2.2 & 1.0 & 2.8 & 1.0 \\
\hline & 181 & 28242 & 7 & 3 & .0 & 12.7 & -3.4 & 2.4 & 1.7 & 1.4 & .7 & $.6 *$ \\
\hline & 181 & 28242 & $B$ & 1 & 14.8 & 8.9 & -3.8 & 1.9 & 3.6 & 1.0 & 5.3 & 1.1 \\
\hline & 181 & 28242 & 8 & 2 & -5.2 & 12.0 & 1.9 & 1.7 & 2.5 & 1.6 & 2.7 & 1.3 \\
\hline & $1 \theta 3$ & 28525 & 1 & 1 & 72.6 & 16.7 & -2.6 & 2.2 & 3.0 & 1.5 & 8.1 & 1.6 \\
\hline & 183 & 28525 & 1 & 2 & 37.0 & 14.0 & -10.9 & 2.7 & 3.5 & 1.6 & 1.1 & $.5 *$ \\
\hline & 183 & 28525 & 2 & 1 & 40.0 & 11.0 & 3.2 & 1.9 & 1.7 & 1.0 & 3.9 & 1.1 \\
\hline & 183 & 28525 & 2 & 2 & 59.4 & 12.4 & .4 & 2.0 & 1.1 & 2.0 & 5.8 & 1.2 \\
\hline & 183 & 28525 & 4 & 1 & 5.3 & 12.1 & $\$ 1.0$ & .9 & -.1 & 1.2 & -.4 & $.4 *$ \\
\hline & 183 & 28525 & 4 & 2 & -13.9 & 11.0 & 1.0 & .1 & 2.1 & 1.4 & -.1 & $.8 * k$ \\
\hline & 183 & 28525 & 5 & 1 & 39.0 & 13.0 & & & 2.9 & 1.7 & .0 & $1.1 *$ \\
\hline & 185 & 28772 & 1 & 1 & 35.0 & 12.0 & 4.6 & 2.0 & 3.9 & 1.2 & -.6 & $1.1 *$ \\
\hline & 185 & 28772 & 1 & 2 & 39.0 & 15.0 & 3.7 & 1.8 & 4.6 & 1.6 & -.5 & $1.0: k$ \\
\hline & 185 & 28772 & 1 & 3 & 23.0 & 13.0 & B. 4 & 2.1 & 7.3 & 1.8 & .6 & $1.3 *$ \\
\hline & 185 & 28772 & 2 & $t$ & 35.0 & 11.0 & 5.7 & 1.7 & 1.7 & .9 & .5 & $1.1 \neq$ \\
\hline & 185 & 28772 & 2 & 2 & 58.0 & 14.0 & & & 1.5 & 1.7 & -.1 & $.8 *$ \\
\hline & 185 & 28772 & 4 & 1 & 51.0 & 16.0 & & & 4.4 & 1.9 & .6 & $1.1 *$ \\
\hline & 185 & 28772 & 4 & 2 & 5.3 & 0.5 & .3 & 1.4 & -.7 & 1.3 & 1.2 & $1.0 \%$ \\
\hline & 185 & 28772 & 5 & 1 & -14.9 & 10.0 & 6.0 & 1.9 & 3.4 & 1.1 & -.6 & $1.1: *$ \\
\hline & 187 & 29101 & 1 & 1 & 49.6 & 15.0 & 1.8 & 1.9 & 2.1 & 1.5 & 2.8 & $\$ .3$ \\
\hline 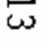 & 187 & 29101 & 1 & 2 & 21.0 & 13.0 & -1.0 & 2.0 & 5.4 & 1.4 & 3.3 & 1.3 \\
\hline & 187 & 29101 & 2 & 1 & 23.5 & 9.8 & -1.2 & 1.6 & 1.2 & .9 & 2.4 & .9 \\
\hline & 187 & 29101 & 3 & 1 & 54.0 & 15.0 & & & 4.0 & 1.5 & 1.3 & $1.0 *$ \\
\hline & 187 & 29101 & 5 & 1 & 21.0 & 8.8 & -.9 & 2.1 & 2.1 & 1.0 & .2 & $.4 *$ \\
\hline & $18 B$ & 29271 & 5 & $t$ & 30.0 & 13.0 & & & 2.3 & 1.5 & 1.0 & $.9 *$ \\
\hline & 189 & 29514 & 1 & 1 & 32.0 & 13.0 & & & .4 & 1.6 & .8 & $1.0 *$ \\
\hline & 189 & 29514 & 1 & 2 & 27.0 & 9.3 & & & .3 & .9 & .0 & $.9: 5$ \\
\hline & 189 & 29514 & 2 & 1 & 12.0 & 12.0 & & & 1.0 & 1.6 & -.1 & $.9 *$ \\
\hline & 189 & 29514 & 2 & 2 & 16.0 & 9.0 & 2.5 & 1.7 & 2.2 & 1.1 & .5 & $.8 *$ \\
\hline & 189 & 29514 & 3 & 1 & 12.0 & 16.0 & & & 4.8 & 1.8 & .6 & $1.4 \%$ \\
\hline & 189 & 29514 & 5 & 1 & 32.0 & 14.0 & & & 3.1 & 1.5 & -.7 & $1.2 *$ \\
\hline & 191 & 29833 & 1 & 1 & 18.0 & 10.0 & 1.4 & 1.4 & 3.6 & 1.4 & .0 & $.9 *$ \\
\hline & 191 & 29833 & 1 & 2 & 10.6 & 13.3 & -1.7 & 2.0 & 2.9 & 1.3 & 5.2 & 1.6 \\
\hline & 191 & 29833 & 2 & 1 & 3.7 & 10.0 & 2.0 & 1.9 & 1.8 & .9 & .2 & $.4 *$ \\
\hline & 191 & 29833 & 2 & 2 & 23.0 & 13.0 & 1.0 & .1 & 2.1 & 1.6 & .0 & $.8 *$ \\
\hline & 191 & 29033 & 3 & 1 & 8.7 & 9.2 & 14.0 & 2.3 & .4 & .9 & -.6 & $1.0 *$ \\
\hline & 191 & 29833 & 4 & 1 & 104.0 & 18.0 & 11.0 & 1.2 & 2.9 & 1.8 & .0 & $1.0 *$ \\
\hline & 191 & 29833 & 4 & 2 & 71.0 & 18.0 & 1.2 & .2 & .2 & 1.6 & -.3 & $1.1: *$ \\
\hline & 191 & 29833 & 5 & 1 & 17.0 & 10.0 & & & 1.7 & .9 & -1.2 & $.8 *$ \\
\hline & 191 & 29833 & $b$ & 1 & -27.9 & 13.0 & 1.2 & 1.9 & .6 & 1.3 & -1.5 & $1.1: k$ \\
\hline & 191 & 29833 & 6 & 2 & .0 & 9.6 & 1.7 & .2 & 1.5 & 1.0 & -.3 & $1.0 *$ \\
\hline
\end{tabular}




\begin{tabular}{|c|c|c|c|c|c|c|c|c|c|c|c|}
\hline STAKE & DISTANCE & SAH & & THO & & & & & & URA & \\
\hline ND. & HETERS & TYPE & REP. & LEVEL & ERROR & LEVEL & ERRDR & LEVEL & ERROR & LEVEL & ERROK \\
\hline 191 & 29833 & 7 & 1 & 28.0 & 13.0 & & & -1.2 & 1.4 & -.1 & $1.1 *$ \\
\hline 191 & 29933 & 7 & 2 & 14.0 & 8.0 & & & -.2 & .7 & .4 & $.9 *$ \\
\hline 191 & 29833 & $B$ & 1 & 19.0 & 8.1 & & & .6 & .9 & 1.1 & $.9 *$ \\
\hline 193 & 30123 & 1 & 1 & 8.7 & 9.2 & & & .8 & .9 & -1.3 & $1.0 *$ \\
\hline 193 & 30123 & 1 & 2 & 45.0 & 12.0 & 1.0 & .1 & 3.2 & 1.1 & .5 & $.9 *$ \\
\hline 193 & 30123 & 2 & 1 & 8.7 & 7.9 & 2.6 & 1.8 & -.5 & 1.0 & 2.2 & 1.0 \\
\hline 193 & 30123 & 2 & 2 & 73.0 & 15.0 & -1.8 & 2.1 & 5.4 & 1.7 & 7.1 & 1.5 \\
\hline 193 & 30123 & 3 & 1 & 8.7 & 9.3 & & & 1.4 & 1.0 & 1.6 & $.9 *$ \\
\hline 193 & 30123 & 4 & 1 & 57.0 & 12.0 & -.9 & 2.0 & 1.1 & 1.1 & 5.0 & 1.2 \\
\hline 195 & 30320 & 1 & 1 & 30.0 & 10.0 & & & 1.6 & 1.0 & -.1 & $1.1 *$ \\
\hline 195 & 30320 & 1 & 2 & 44.0 & 17.0 & & & 3.8 & 1.8 & -.7 & $1.0 *$ \\
\hline 195 & 30320 & 2 & 1 & 12.0 & 9.0 & .6 & 1.6 & 1.0 & 1.0 & .4 & $.9 *$ \\
\hline 195 & 30320 & 2 & 2 & 25.0 & 11.0 & & & 2.9 & 1.1 & 1.6 & .94 \\
\hline 195 & 30320 & 3 & 1 & 115.0 & 23.0 & 8.1 & 2.5 & 13.0 & 2.6 & 2.0 & $1.5 *$ \\
\hline 195 & 30320 & 5 & 1 & 30.0 & 9.1 & & & .8 & .8 & -.6 & $.9 *$ \\
\hline 197 & 30641 & 1 & 1 & 7.4 & 8.0 & -.4 & 1.7 & 1.6 & .9 & 2.8 & .9 \\
\hline 197 & 30614 & 1 & 2 & 122.2 & 19.0 & -.3 & 2.3 & 3.6 & 1.6 & 9.8 & 1.7 \\
\hline 197 & 30641 & 2 & 1 & 37.0 & 13.0 & & & 2.3 & 1.7 & -.1 & $.8 *$ \\
\hline 198 & 30019 & 4 & 1 & 43.0 & 15.0 & & & 5.4 & 1.8 & -.7 & $.9 *$ \\
\hline 198 & 30019 & 5 & 1 & 81.0 & 19.0 & 1.8 & .5 & 2.3 & 2.0 & -1.3 & $1.1 *$ \\
\hline 199 & 30970 & 1 & 1 & 34.0 & 12.0 & .1 & 2.0 & 3.3 & 1.3 & 5.7 & 1.3 \\
\hline 199 & 30970 & $i$ & 2 & 16.0 & B.5 & & & 1.8 & .9 & -.4 & $.7 *$ \\
\hline 199 & 30970 & 2 & 1 & 10.7 & 12.4 & -1.1 & 1.9 & .4 & 1.4 & .3 & $.5 *$ \\
\hline 199 & 30970 & 2 & 2 & 89.0 & 19.0 & 1.2 & .1 & 4.4 & 2.0 & .0 & $1.1 *$ \\
\hline 199 & 30970 & 3 & 1 & 67.0 & 14.0 & & & 6.2 & 1.4 & -.3 & $1.3 *$ \\
\hline 201 & 31239 & 1 & 1 & 27.0 & 12.0 & & & .0 & 1.5 & .2 & $.9 *$ \\
\hline 201 & 31239 & 1 & 2 & 14.8 & 9.3 & -1.1 & 1.9 & 3.6 & 1.1 & 6.3 & 1.2 \\
\hline 201 & 31239 & 1 & 3 & 24.0 & 8.8 & & & .8 & .9 & 1.3 & $1.0 *$ \\
\hline 201 & 31239 & 2 & 1 & -10.9 & 7.3 & & & 2.9 & 1.1 & .4 & $.9 *$ \\
\hline 201 & 31239 & 4 & $i$ & 18.0 & 12.0 & 2.6 & .5 & 4.1 & 1.3 & 2.0 & $.9 *$ \\
\hline 209 & 31239 & 6 & 1 & -17.9 & 11.0 & & & 6.3 & 1.6 & .2 & $1.1 *$ \\
\hline 201 & 31239 & 6 & 2 & 34.0 & 14.0 & 1.4 & .2 & 2.3 & 1.4 & 1.0 & 1.0 \\
\hline 201 & 31239 & 7 & 1 & 27.0 & 11.0 & 1.4 & .2 & 2.3 & 1.0 & -1.2 & $.9 *$ \\
\hline 201 & 31239 & 7 & 2 & 154.1 & 21.7 & -4.1 & 2.4 & 6.7 & 1.9 & 13.0 & 1.9 \\
\hline 201 & 31239 & 7 & 3 & -14.9 & 7.0 & & & .4 & .8 & .7 & $.7 *$ \\
\hline 201 & 31239 & 8 & 1 & 23.0 & 12.0 & & & .8 & 1.1 & -.1 & $.9 *$ \\
\hline 201 & 31239 & $B$ & 2 & 22.0 & 8.0 & 1.9 & 1.6 & 1.4 & .8 & -.3 & $1.0 *$ \\
\hline 201 & 31239 & $B$ & 3 & -1.7 & 11.0 & & & -.5 & 1.2 & .8 & $.9 *$ \\
\hline 203 & 31479 & 1 & 1 & 9.9 & 9.7 & 1.0 & 1.7 & 3.7 & 1.1 & .2 & $.4 *$ \\
\hline 203 & 31479 & 1 & 2 & 22.0 & 9.6 & & & 3.3 & 1.0 & 1.1 & $1.0 *$ \\
\hline 203 & 31479 & 2 & 1 & 24.7 & 12.5 & 6.1 & .5 & .4 & 1.4 & -.4 & $.4 *$ \\
\hline
\end{tabular}




\begin{tabular}{|c|c|c|c|c|c|c|c|c|c|c|c|}
\hline STAKE & DISTANCE & SAH & & THO & & & & & & URA & \\
\hline No. & METERS & TYFE & REF. & LEVEL & ERROR & LEVEL & ERROR & LEVEL & ERROR & LEVEL & ERROR \\
\hline 203 & 31479 & 2 & 2 & 35.0 & 14.0 & 1.3 & .3 & 2.3 & 1.5 & 1.4 & $1.1 *$ \\
\hline 203 & 31479 & 3 & 1 & 50.0 & 16.0 & 7.2 & 1.9 & 3.8 & 1.9 & 1.6 & $1.2 *$ \\
\hline 205 & 31745 & 1 & 1 & 31.0 & 11.0 & 3.3 & 1.6 & 2.1 & 1.0 & .2 & $.9 *$ \\
\hline 205 & $3 \uparrow 745$ & 1 & 2 & 14.0 & 10.0 & 2.3 & 1.4 & -1.2 & 1.4 & .4 & $.9 *$ \\
\hline 205 & 31745 & 2 & 1 & 32.0 & 12.0 & 4.6 & 1.5 & 1.9 & 1.4 & -.1 & $1.0 \%$ \\
\hline 205 & 31745 & 2 & 2 & 26.0 & 11.0 & & & 2.6 & .9 & -.3 & $.9 *$ \\
\hline 205 & 31745 & 4 & 1 & 30.0 & 10.0 & 1.6 & 1.0 & .4 & .8 & -.4 & $.9 *$ \\
\hline 207 & 32052 & 1 & 1 & 19.0 & 12.0 & 3.2 & 1.8 & 2.7 & 1.3 & 1.6 & 1.1 \\
\hline 207 & 32052 & 2 & 1 & 32.2 & 10.9 & -2.2 & 2.2 & .9 & 2.0 & 5.7 & 1.2 \\
\hline 207 & 32052 & 2 & 2 & 33.7 & 15.3 & -.3 & 1.7 & .2 & 1.4 & 4.4 & 1.4 \\
\hline 207 & 32052 & 3 & 1 & 37.1 & 10.6 & -.3 & 2.0 & 2.1 & 1.1 & 3.0 & 1.1 \\
\hline 209 & 32371 & 1 & 1 & 28.3 & 13.0 & -1.6 & 1.6 & -.1 & 1.5 & 5.0 & 1.3 \\
\hline 209 & 32371 & 1 & 2 & 17.7 & 10.9 & -1.5 & 1.5 & 2.7 & 1.4 & 4.1 & 1.2 \\
\hline 209 & 32371 & 2 & 1 & 24.8 & 9.9 & .7 & $1 . \theta$ & 3.3 & 1.1 & 5.3 & 1.0 \\
\hline 209 & 32371 & 2 & 2 & 8.9 & 12.4 & -.7 & 1.7 & 3.8 & 1.5 & 3.1 & 1.3 \\
\hline 209 & 32371 & 4 & 1 & 23.0 & 8.3 & -.5 & 1.7 & 1.0 & .8 & 4.2 & 1.0 \\
\hline 209 & 32371 & 9 & 1 & 26.0 & 10.7 & 2.3 & 3.1 & 1.4 & 1.1 & 16.7 & 1.6 \\
\hline 211 & 32695 & 1 & 1 & 18.6 & 10.3 & -3.6 & 1.8 & 1.8 & 1.0 & .5 & $.4 *$ \\
\hline 211 & 32695 & 2 & 1 & 14.0 & 11.0 & -1.6 & 1.6 & 1.9 & 1.2 & .2 & $.5 *$ \\
\hline 211 & 32695 & 6 & 1 & 5.3 & 12.0 & & & 2.3 & 1.4 & 1.0 & $1.0 *$ \\
\hline 211 & 32695 & 7 & 1 & 8.7 & 8.1 & & & .7 & .8 & .4 & $.9 *$ \\
\hline 211 & 32695 & 7 & 2 & -4.8 & 7.6 & 1.6 & 1.7 & -.0 & .8 & 3.1 & .9 \\
\hline 211 & 32695 & 7 & 3 & -16.9 & 6.5 & 4.7 & 1.2 & .4 & .9 & -.0 & $.3 *$ \\
\hline 213 & 33053 & 1 & 1 & 21.0 & 8.1 & .7 & 2.1 & 1.1 & .9 & .4 & $.3 *$ \\
\hline 213 & 33053 & 2 & 1 & 51.0 & 13.0 & 4.2 & 1.7 & 1.9 & 1.4 & .1 & $3.6 \%$ \\
\hline 215 & 33353 & 1 & 1 & 14.0 & 13.0 & 4.0 & 1.7 & 3.3 & 1.7 & 1.0 & $.9 *$ \\
\hline 215 & 33353 & 2 & 1 & 32.0 & 17.0 & & & -.7 & 1.8 & -.9 & $1.0 *$ \\
\hline 217 & 33684 & 1 & 1 & 11.0 & 12.0 & & & 1.5 & 1.1 & .4 & $1.0 *$ \\
\hline 217 & 33684 & 1 & 2 & 55.0 & 14.0 & .8 & .1 & 5.0 & 1.6 & .2 & $.9 *$ \\
\hline 219 & 33960 & 1 & 1 & 26.5 & 13.6 & 1.5 & 2.0 & 2.9 & 1.2 & 6.6 & 1.4 \\
\hline 219 & 33960 & 2 & 1 & 12.0 & 11.0 & -.1 & 1.7 & .6 & 1.0 & .2 & $.4 *$ \\
\hline 219 & 33960 & 3 & 1 & 40.0 & 11.0 & .8 & .1 & 2.6 & 1.0 & .2 & $1.0 *$ \\
\hline 221 & 34327 & 1 & 1 & 23.0 & 14.0 & & & 2.1 & 1.6 & -.7 & $.7 *$ \\
\hline 221 & 34327 & 1 & 2 & 37.1 & 8.6 & -1.8 & 1.7 & 2.5 & .9 & 4.5 & 1.0 \\
\hline 221 & 34327 & 6 & 1 & -3.6 & 10.0 & 1.2 & .2 & 1.1 & 1.1 & .5 & $.9 *$ \\
\hline 221 & 34327 & 7 & 1 & 19.8 & 7.8 & -1.6 & 2.1 & 2.9 & .9 & .4 & $.3 *$ \\
\hline 221 & 34327 & 7 & 2 & 9.9 & 8.4 & & & 1.9 & .9 & .9 & $.8 *$ \\
\hline 221 & 34327 & 7 & 3 & 16.0 & 12.0 & & & -2.6 & 1.5 & -.1 & $.8 *$ \\
\hline 221 & 34327 & 8 & 1 & 1.2 & 7.5 & & & 2.3 & .9 & 1.3 & $.9: k$ \\
\hline 223 & 34639 & 1 & 1 & 11.0 & 13.0 & .4 & .2 & 4.2 & 1.5 & .2 & $1.0 *$ \\
\hline 223 & 34639 & 1 & 2 & 5.3 & 12.0 & .6 & .1 & -.1 & 1.6 & -.1 & $.7 *$ \\
\hline
\end{tabular}




\begin{tabular}{|c|c|c|c|c|c|c|c|c|c|c|c|}
\hline STAKE & DISTAKCE & SAHI & LE & THO & & & & & & & \\
\hline No. & KETERS & TYF'E & REP. & LEVEL & ERKOR' & LEVEL & ERKOR & IEVEL & ERKOK & LFUEL & ERRUK \\
\hline 223 & 34639 & 2 & 1 & 30.0 & 13.0 & & & 1.7 & 1.5 & -1.1 & $.8 *$ \\
\hline 225 & 34939 & 1 & 1 & 27.0 & 13.0 & 1.7 & 1.6 & 1.7 & 1.6 & -.7 & $1.0 *$ \\
\hline 225 & 34939 & 1 & 2 & 8.9 & 13.0 & 1.7 & 1.5 & 2.1 & 1.3 & .2 & $1.1 *$ \\
\hline 225 & 34939 & 2 & 1 & 7.4 & 9.7 & & & 3.0 & 1.0 & -.8 & $.9 *$ \\
\hline 225 & 34939 & 3 & 1 & 77.0 & 15.0 & & & 2,2 & 1.2 & $\$ .3$ & $1.0 *$ \\
\hline 225 & 34939 & 3 & 2 & 41.0 & 12.0 & 5.5 & 1.7 & 1.1 & $\uparrow .1$ & .9 & $1.0 *$ \\
\hline 225 & 34939 & 4 & 1 & 103.0 & 19.0 & & & 5.6 & 1.9 &.$\theta$ & $1.2 *$ \\
\hline 227 & 35215 & 1 & 1 & 64,0 & 13.0 & -7.5 & 2.6 & 3.4 & 1.1 & .8 & $.5 * k$ \\
\hline 227 & 35215 & 1 & 2 & 44.0 & 14.0 & .8 & .1 & 1.5 & 1.4 & .8 & $1.0 *$ \\
\hline 229 & 35477 & 1 & 1 & 44.3 & 14.1 & -5.4 & 2.4 & 5.2 & 1.6 & .7 & $.4 *$ \\
\hline 229 & 35477 & 1 & 2 & 25.0 & 9.3 & 8.0 & .8 & 1.8 & .9 & -.3 & $.3 *$ \\
\hline 229 & 35477 & 1 & 3 & 18.0 & 13.0 & .8 & .1 & 2.7 & 1.5 & .4 & $.9 *$ \\
\hline 231 & 35784 & 1 & 1 & 35.0 & 10.0 & .8 & .1 & 1.2 & 1.0 & .5 & $.7 *$ \\
\hline 231 & 35784 & 1 & 2 & 101.5 & 14.8 & -15.4 & 3.2 & 6.2 & 1.3 & 1.5 & $.5 *$ \\
\hline 231 & 35784 & 2 & 1 & 6.2 & 9.3 & .6 & .1 & 2.1 & .9 & -.1 & $.8: k$ \\
\hline 231 & 35784 & 6 & 1 & -15.9 & 10.0 & & & 1.9 & 1.1 & -1.2 & $1.1 *$ \\
\hline 231 & 35784 & 7 & 1 & 8.9 & 11.0 & 1.3 & .4 & 2.7 & 1.5 & .2 & $1.0 *$ \\
\hline 231 & 35784 & 7 & 2 & 1.8 & 9.5 & & & 1.7 & 1.4 & -1.3 & $.8: k$ \\
\hline 231 & 35784 & 8 & 1 & 15.0 & 8.2 & .6 & .1 & 1.9 & .9 & .4 & . $\theta *$ \\
\hline 231 & 35784 & 8 & 2 & 43.0 & 13.0 & & & -.1 & 1.5 & 1.2 & $.9 *$ \\
\hline 231 & 35784 & 8 & 3 & 8.9 & 11.0 & 1.3 & .4 & 2.7 & 1.5 & .2 & $1.0 *$ \\
\hline 233 & 36118 & 1 & 1 & 143.0 & 22.0 & & & 4.6 & 2.0 & .8 & $1.4 *$ \\
\hline 233 & 36110 & 2 & 1 & 41.0 & 16.0 & 1.3 & .2 & 3.1 & 1.7 & .8 & $1.0 *$ \\
\hline 235 & 36435 & 1 & 1 & 26.0 & 10.0 & 9.0 & 2.0 & 3.4 & 1.1 & 1.6 & $1.0 *$ \\
\hline 235 & 36435 & 2 & 1 & 35.0 & 11.0 & & & 3.6 & 1.1 & .2 & 1.04 \\
\hline 237 & 36850 & 2 & 1 & 16.0 & 9.5 & -5.5 & 2.0 & .8 & 1.0 & .5 & -4: \\
\hline 237 & 36850 & 4 & 1 & 35.0 & 16.0 & & & 4.4 & 1.8 & -1.1 & $1.2 *$ \\
\hline 237 & 36850 & 7 & 3 & -3.4 & 9.7 & -.8 & 1.8 & -1.7 & 1.3 & .3 & $.4 *$ \\
\hline 239 & 37191 & 1 & 1 & 21.0 & 11.0 & 4.9 & 1.8 & 5.1 & 1.2 & 2.0 & $1.1 *$ \\
\hline 239 & 37191 & 1 & 2 & 90.0 & 21.0 & 2.9 & .5 & 4.8 & 2.0 & -.7 & $1.3 *$ \\
\hline 239 & 37191 & 2 & 1 & 41.0 & 14.0 & 3.0 & 1.8 & 4.0 & 1.4 & .1 & $.4 *$ \\
\hline 239 & 37191 & 2 & 2 & 110.0 & 19.0 & 1.5 & .2 & 4.2 & 1.8 & -.1 & $1.3 *$ \\
\hline 241 & 37977 & 1 & 1 & 28.0 & 11.0 & & & 2.5 & 1.0 & .4 & $.9 *$ \\
\hline 241 & 37477 & 1 & 2 & 17.0 & 9.7 & & & 1.5 & 1.1 & -.1 & $.9 *$ \\
\hline 241 & 37477 & 2 & 1 & 26.0 & 8,3 & & & 1.8 & .9 & .9 & $.9 *$ \\
\hline 241 & 37477 & 2 & 2 & 31.8 & 13.5 & 1.0 & 2.1 & 2.5 & 1.5 & $5 . ?$ & 1.4 \\
\hline 241 & 37477 & 6 & 1 & 12.0 & 8.0 & 1.1 & .1 & 2.3 & 1.0 & .0 & $.9 *$ \\
\hline $24 !$ & 37477 & 7 & 1 & 1.2 & 7.9 & -.9 & 1.6 & 2.3 & .9 & .2 & $.4 *$ \\
\hline 241 & 37477 & 7 & 2 & 3.5 & 9.0 & 2.9 & 1.3 & -.3 & $1 . t$ & .0 & $.3 *$ \\
\hline 241 & 37477 & 7 & 3 & -11.0 & 7.7 & 2.1 & 2.0 & 2.4 & .9 & 1.8 & 1.0 \\
\hline 241 & 37477 & 8 & 1 & 7.8 & 12.5 & 1.6 & 1.7 & 1.9 & 1.4 & 3.2 & 1.3 \\
\hline
\end{tabular}




\begin{tabular}{|c|c|c|c|c|c|c|c|c|c|c|c|}
\hline STAKE & DISTANCE & SAH & F'LE & THO & & & & & & URA & \\
\hline NO. & METERS & TYFE & REF. & LEVEL & ERROK & LEVEL & ERROR & LEVEL & EKkOR & LEVEL & EAROR \\
\hline 241 & 37477 & $\mathrm{~g}$ & 2 & -11.9 & 10.0 & & & .6 & 1.0 & -.9 & $1.1 *$ \\
\hline 241 & 37477 & 8 & 3 & 14.9 & 7.0 & -.2 & 1.3 & 1.7 & .9 & -.3 & $.4 *$ \\
\hline 243 & 37813 & 1 & 1 & 23.0 & 13.0 & & & 3.3 & 1.7 & 1.2 & $1.0 *$ \\
\hline 243 & 37813 & 2 & 1 & 26.0 & 9.7 & .9 & .2 & 2.3 & .8 & .4 & $.9 . *$ \\
\hline 243 & 37813 & 2 & 2 & 39.0 & 12.0 & -2.2 & 2.3 & .4 & 1.5 & .5 & $.4: *$ \\
\hline 243 & 37813 & 3 & 1 & 21.0 & 9.7 & .8 & .1 & .8 & 1.4 & .6 & $.8: k$ \\
\hline 243 & 37813 & 3 & 2 & 21.2 & 12.0 & -.9 & 1.8 & 1.0 & 1.3 & .3 & $.4 *$ \\
\hline 245 & 38096 & 2 & 1 & 46.0 & 13.0 & 1.7 & 1.8 & -1.4 & 1.5 & 1.4 & $1.0 \%$ \\
\hline 247 & 38410 & 1 & 1 & 30.0 & 12.0 & .9 & .1 & .2 & 1.5 & .0 & $.9 *$ \\
\hline 247 & 38410 & 2 & 1 & 23.1 & 10.3 & -.7 & 2.1 & 3.3 & 1.1 & 6.5 & 1.2 \\
\hline 249 & 38715 & $t$ & 1 & 32.1 & 10.6 & -3.0 & 2.2 & 3.2 & .9 & .4 & $.4 *$ \\
\hline 249 & 38715 & 2 & 1 & 60.2 & 14.2 & 1.3 & 1.9 & 2.1 & 1.3 & 4.8 & 1.3 \\
\hline 251 & 39005 & 1 & 1 & 17.3 & 8.9 & 3.0 & 1.6 & 1.8 & 1.0 & 3.8 & 1.1 \\
\hline 251 & 39005 & 1 & 2 & 1.8 & 12.0 & & & 2.9 & 1.6 & .2 & $1.1 \%$ \\
\hline 251 & 39005 & 2 & 1 & 25.0 & 12.0 & 2.6 & 1.6 & 2.3 & 1.5 & -.3 & $1.0 *$ \\
\hline 251 & 39005 & 6 & 2 & 3.7 & 9.5 & 2.8 & .3 & 1.4 & .9 & -1.5 & $1.0 *$ \\
\hline 251 & 39005 & 6 & 3 & -8.6 & 8.3 & 1.0 & .1 & 1.8 & 1.0 & -.4 & $.9 *$ \\
\hline 253 & 39219 & 1 & 1 & 9.9 & 8.6 & .9 & 1.6 & 1.2 & .8 & 2.0 & .8 \\
\hline 253 & 39291 & 1 & 2 & 40.7 & 13.3 & -.4 & 1.9 & 6.5 & 1.6 & 6.0 & 1.4 \\
\hline 253 & 39291 & 2 & 1 & 28.3 & 12.0 & -.2 & 1.9 & 3.6 & 1.3 & 4.7 & 1.3 \\
\hline 253 & 39291 & 2 & 2 & 7.4 & 7.4 & 1.1 & 2.1 & .1 & 1.1 & 5.8 & $\uparrow .1$ \\
\hline 255 & 39663 & 1 & 1 & 3.5 & 13.0 & 2.0 & 1.7 & 3.3 & 1.5 & -.9 & $.9 *$ \\
\hline 257 & 39920 & 1 & 1 & -2.4 & 8.7 & .5 & 1.9 & 1.1 & 1.0 & 3.9 & 1.0 \\
\hline 257 & 39920 & 2 & 1 & 40.7 & 13.8 & -.2 & 1.7 & 1.7 & 1.3 & 4.8 & 1.3 \\
\hline 259 & 40225 & 1 & 1 & 30.1 & 15.1 & 1.5 & 1.6 & 1.9 & $\uparrow .4$ & 2.0 & 1.3 \\
\hline 259 & 40225 & 1 & 2 & 7.0 & 12.8 & -1.6 & 1.7 & 1.0 & 1.2 & 4.9 & 1.3 \\
\hline 259 & 40225 & 2 & 1 & 49.5 & 12.1 & -.8 & 2.2 & 1.4 & 1.0 & 7.7 & 1.2 \\
\hline 261 & 40551 & 1 & 1 & 47.8 & 13.8 & -6.3 & 2.3 & 1.7 & 1.3 & .7 & $.4 *$ \\
\hline 261 & 40551 & 1 & 2 & $\$ 6.0$ & 10.0 & & & -1.6 & 1.4 & -.1 & $.8 *$ \\
\hline 261 & 40551 & 2 & 1 & 61.9 & 12.3 & -.0 & 1.7 & 2.6 & 1.2 & $4 . h$ & 1. \\
\hline 261 & 40551 & 6 & 1 & -8.6 & 9.5 & 1.1 & .1 & .0 & .9 & .4 & $1.0 *$ \\
\hline 261 & 40551 & 6 & 2 & -7.3 & 8.2 & & & .8 & 1.1 & -1.3 & $.9 *$ \\
\hline 261 & 40551 & 7 & 1 & 44.3 & 13.1 & -1.3 & 1.6 & 2.5 & 1.2 & 3.7 & 1.1 \\
\hline 261 & 40551 & 7 & 2 & 17.3 & 10.0 & 2.7 & 1.6 & 2.5 & .9 & 1.7 & .9 \\
\hline 261 & $4055 t$ & $B$ & 1 & 30.9 & 8.5 & 1.4 & 1.8 & .1 & .9 & 3.7 & .7 \\
\hline 261 & 40551 & 8 & 2 & 7.4 & 8.9 & & & 1.4 & .9 & -.4 & $.8 \%$ \\
\hline 263 & 40897 & i & 1 & 22.0 & 11.0 & & & 2.5 & 1.0 & 1.3 & $.8 *$ \\
\hline 263 & 40897 & 1 & 2 & 30.1 & 12.9 & -9.8 & 2.6 & 5.2 & 1.5 & 1.0 & $.5 \%$ \\
\hline 263 & 40897 & 2 & 1 & 41.0 & 14.0 & & & 1.5 & 1.5 & .8 & $.9: k$ \\
\hline 263 & 40897 & 2 & 2 & 21.3 & 10.9 & -.8 & 1.6 & .6 & 1.3 & 3.8 & $1 . ?$ \\
\hline 265 & 41171 & 1 & 1 & 7.4 & 10.0 & 3.6 & 1.4 & 1.7 & 1.0 & -1.9 & $1.0 *$ \\
\hline
\end{tabular}




\begin{tabular}{|c|c|c|c|c|c|c|c|c|c|c|c|}
\hline STAKE & IISTANCE & SAAI & & THOF & & & & & & UR: & \\
\hline no. & METERS & TYPE & REP. & LEVEL & ERKOR & LEVEL & ERROK & LEVEL & ERROR & LEVEL & ERROR \\
\hline 265 & 41171 & 1 & 2 & 30.0 & 14.0 & .6 & 1.5 & 2.9 & 1.7 & .8 & $1.2 *$ \\
\hline 265 & 41171 & 2 & i & 25.0 & 9.0 & 2.7 & 1.7 & 2.6 & 1.1 & .7 & $1.0 *$ \\
\hline 267 & 41404 & 1 & 1 & 21.0 & 12.0 & & & 2.5 & 1.5 & .2 & $1.0 *$ \\
\hline 267 & 11404 & 1 & 2 & 50.0 & 11.0 & .7 & .2 & 3.6 & 1.1 & 1.8 & $.9 *$ \\
\hline 267 & 41404 & 2 & 1 & 17.0 & 11.0 & & & 2.3 & 1.0 & -1.7 & $.9 *$ \\
\hline 267 & 41404 & 2 & 2 & 12.0 & 11.0 & .3 & 1.3 & .8 & 1.2 & -.3 & $.8 *$ \\
\hline 269 & 41774 & 1 & $i$ & 10.6 & 9.7 & .6 & 1.6 & .4 & 1.0 & .2 & $.3 *$ \\
\hline 269 & 41774 & 1 & 2 & 1.2 & 9.2 & 5.2 & 1.8 & 2.2 & 1.1 & .5 & $1.2 *$ \\
\hline 269 & 41774 & 2 & 1 & 21.3 & 14.4 & 7.8 & 1.2 & 2.3 & 1.4 & -.2 & $.5 *$ \\
\hline 271 & 42184 & 1 & 1 & 14.0 & 13.0 & & & 1.0 & 1.4 & -.7 & $.9 *$ \\
\hline 271 & 42184 & 1 & 2 & 44.0 & 14.0 & .9 & .1 & 1.0 & 1.5 & .2 & $1.2 *$ \\
\hline 271 & 42184 & 2 & $i$ & 18.0 & 13.0 & .8 & .1 & 3.6 & 1.5 & 1.4 & $1.0 *$ \\
\hline 271 & 12184 & 2 & 2 & 47.0 & 11.3 & 1.6 & 1.9 & 1.1 & .9 & 2.8 & 1.0 \\
\hline 271 & 12184 & 6 & 1 & 11.0 & 8.7 & & & .6 & 1.1 & .0 & $1.0 *$ \\
\hline 271 & 42184 & 6 & 2 & 3.5 & 13.0 & 1.5 & .2 & 2.1 & 1.7 & -1.1 & $1.0 *$ \\
\hline 271 & 42184 & 7 & 1 & 26.6 & 11.3 & -.3 & 1.9 & 4.0 & 1.3 & 6.1 & 1.3 \\
\hline 271 & 42184 & 7 & 2 & 38.4 & 10.3 & -14.5 & 2.6 & .1 & .9 & 1.1 & $.4 *$ \\
\hline 271 & 42184 & 7 & 3 & 35.4 & 13.5 & 9.1 & 2.0 & 2.1 & 1.5 & .6 & $.5 *$ \\
\hline 271 & 42184 & 8 & 1 & 25.0 & 9.1 & 2.7 & 1.5 & 1.5 & 1.0 & -1.0 & $.8 *$ \\
\hline 271 & 42184 & 8 & 2 & 18.6 & 8.8 & -1.5 & 2.1 & 2.1 & 1.0 & .6 & $.3 *$ \\
\hline 271 & 12184 & 8 & 3 & .0 & 10.0 & & & .8 & 1.4 & -.9 & .74 \\
\hline 273 & 12554 & 1 & 1 & 1.8 & 10.0 & & & 1.5 & 1.3 & .4 & $1.0 \%$ \\
\hline 273 & 42554 & 1 & 3 & 47.8 & 15.9 & 3.1 & 1.9 & 1.7 & 1.4 & 3.9 & 1.4 \\
\hline 273 & 42554 & 2 & 1 & 2.5 & 9.6 & .8 & .1 & 2.2 & 1.1 & .4 & 1.0 \\
\hline 275 & 42871 & 1 & 1 & 12.0 & 13.0 & 2.0 & 1.5 & 1.7 & 1.3 & -1.7 & $1.0 *$ \\
\hline 275 & 42871 & 2 & 1 & 11.0 & 11.0 & .3 & 1.9 & 2.8 & 1.0 & 4.1 & 1.1 \\
\hline 277 & 13195 & 1 & 1 & 35.9 & 11.4 & -1.3 & 2.1 & 3.7 & 1.1 & 6.3 & 1.1 \\
\hline 277 & 43195 & 1 & 2 & 60.2 & 13.9 & -.4 & 1.9 & .0 & 1.6 & 5.7 & 1.4 \\
\hline 277 & 43195 & 2 & $i$ & 14.8 & 9.1 & .6 & 1.8 & 1.4 & 1.1 & 3.4 & 1.1 \\
\hline 279 & 43382 & 1 & $i$ & 38.4 & 9.7 & -1.9 & 2.0 & 4.5 & 1.0 & 5.3 & 1.1 \\
\hline 279 & 43382 & 2 & $i$ & 31.9 & 11.8 & -2.1 & 1.7 & 1.4 & 1.2 & 3.6 & 1.3 \\
\hline 281 & 43723 & $t$ & 1 & 31.8 & 14.6 & 1.6 & 2.0 & 1.3 & 1.4 & 5.7 & 1.4 \\
\hline 281 & 43723 & 1 & 2 & 17.7 & 15.0 & -.5 & 1.7 & 3.1 & 1.6 & 3.4 & 1.4 \\
\hline 281 & 43723 & 2 & 1 & 70.8 & 16.9 & .4 & 2.2 & 4.3 & 1.4 & 7.4 & 1.0 \\
\hline 281 & 43723 & 6 & $t$ & 14.0 & 12.0 & 2.0 & 2.1 & 1.7 & 1.4 & 3.4 & 1.4 \\
\hline 281 & 43723 & 6 & 2 & 7.1 & 10.0 & 1.2 & 1.5 & 1.5 & 1.2 & 1.7 & 1.2 \\
\hline 281 & 43723 & 7 & $i$ & 12.0 & 14.0 & .4 & 1.9 & 4.0 & 1.5 & 4.7 & 1.4 \\
\hline 281 & 43723 & 7 & 2 & 5.3 & 13.4 & -2.1 & 1.9 & 1.9 & 1.5 & 5.3 & 1.4 \\
\hline 281 & 43723 & 7 & 3 & -2.4 & 7.6 & -1.5 & 1.7 & 1.0 & .9 & 3.4 & 1.1 \\
\hline 281 & 43723 & 8 & 1 & 38.4 & 11.4 & 2.2 & 1.8 & 3.9 & 1.1 & 2.4 & 1.2 \\
\hline 281 & 43723 & 8 & 2 & 7.4 & 8.4 & 2.3 & 1.4 & .8 & .9 & 1.2 & .9 \\
\hline
\end{tabular}




\begin{tabular}{|c|c|c|c|c|c|c|c|c|c|c|c|}
\hline STAKE & DISTANCE & SAK & PLE & THO & & & & & & URA & \\
\hline no. & NETERS & TYPE & REP. & LEVEL & ERROR & LEVEL & ERROR & LEVEL & ERROR & LEVEL & EKROR \\
\hline 281 & 43723 & 8 & 3 & 13.6 & 8.7 & 1.1 & 1.7 & .8 & .9 & 2.4 & .9 \\
\hline 283 & 41138 & $i$ & $i$ & 34.0 & 15.0 & -2.8 & 2.0 & 4.0 & 1.3 & 6.7 & 1.6 \\
\hline 283 & 44138 & 1 & 2 & 28.5 & 10.6 & -1.3 & 1.9 & 1.4 & 1.0 & 6.3 & 1.2 \\
\hline 283 & 44138 & 2 & 1 & 70.8 & 14.6 & -.5 & 2.2 & 3.3 & 1.4 & 7.2 & 1.4 \\
\hline 285 & 14371 & 1 & 1 & 51.0 & 15.0 & 3.7 & $\$ .8$ & 3.6 & 1.6 & .8 & $.9 *$ \\
\hline 285 & 44371 & 2 & 1 & 17.0 & 9.0 & 1.6 & 1.4 & 1.1 & 1.0 & -1.3 & .94 \\
\hline 287 & 44745 & 1 & 1 & 57.0 & 16.0 & -.9 & 2.1 & 3.8 & 1.5 & 7.4 & 1.5 \\
\hline 287 & 14745 & 1 & 2 & 48.0 & 16.0 & -.9 & 2,3 & 2.1 & 1.6 & 19.0 & 1.7 \\
\hline 287 & 44745 & 2 & 1 & 41.0 & 12.0 & -.7 & 1.9 & 2.2 & 1.1 & 3.8 & 1.2 \\
\hline 289 & 45052 & 1 & 1 & 63.7 & 17.5 & -1.8 & 2.3 & 4.2 & 1.8 & 10.7 & 1.7 \\
\hline 289 & 45052 & 2 & 1 & 129.0 & 16.0 & -4.3 & 2.6 & 5.4 & 1.4 & 11.0 & 1.5 \\
\hline 291 & 45376 & 1 & 1 & 52.0 & 12.6 & 1.8 & 2.4 & 5.0 & 1.3 & 7.4 & 1.3 \\
\hline 291 & 45376 & 1 & 2 & 63.1 & 14.1 & 3.5 & 2.3 & 4.4 & 1.2 & 0.0 & 1.3 \\
\hline 291 & 45376 & 1 & 3 & 10.0 & 0.7 & .5 & 1.9 & 1.6 & .9 & 3.9 & 1.1 \\
\hline 291 & 45376 & 2 & 1 & 109.8 & 19.7 & -1.4 & 2.5 & 4.4 & 2.0 & 11.8 & 1.9 \\
\hline 291 & 45376 & 6 & 1 & .0 & 9.3 & 2.5 & 1.7 & 1.4 & 1.0 & 3.0 & 1.0 \\
\hline 291 & 45376 & 7 & 1 & 58.0 & 16.0 & 2.7 & 2.2 & 3.8 & 1.6 & 7.4 & 1.6 \\
\hline 291 & 45376 & 9 & 1 & 96.0 & 15.0 & .4 & 2.3 & 5.1 & 9.3 & 8.7 & 1.4 \\
\hline 291 & 45376 & 8 & 2 & -13.5 & 9.2 & -.1 & 1.8 & 1.0 & .9 & 3.3 & 1.1 \\
\hline 291 & 45376 & 8 & 3 & 19.0 & 7.4 & 2.9 & 2.1 & 2.7 & 1.0 & 4.6 & 1.0 \\
\hline 292 & 45548 & 1 & 1 & 35.0 & 13.0 & 4.0 & 1.5 & 3.8 & 1.4 & .0 & $.9 *$ \\
\hline 293 & 45642 & 1 & 1 & 7.4 & 7.8 & .7 & .8 & .4 & .9 & 3.9 & 1.0 \\
\hline 293 & 45642 & 1 & 2 & 12.0 & 10.0 & 3.2 & 1.8 & 2.6 & 1.1 & 2.5 & 1.1 \\
\hline 293 & 45642 & 2 & 1 & 26.0 & 12.0 & -1.3 & 1.6 & 1.9 & 1.2 & 3.3 & 1.2 \\
\hline 295 & 45997 & 1 & 1 & 85.0 & 12.0 & 6.8 & 2.0 & 4.2 & 1.1 & -.1 & $1.0 \%$ \\
\hline 295 & 45997 & 1 & 2 & 12.0 & 9.0 & 4.9 & 1.7 & 3.0 & 1.1 & .2 & $.9 *$ \\
\hline 295 & 45997 & 2 & 1 & 1.3 & 12.0 & 6.6 & 1.9 & 6.2 & 1.2 & 1.1 & $1.0 *$ \\
\hline 295 & 45997 & 3 & 1 & 23.0 & 16.0 & 1.1 & 2.3 & 7.1 & 1.9 & 8.1 & 1.7 \\
\hline 295 & 45997 & 3 & 2 & 25.0 & 13.0 & & & 3.6 & 1.6 & .6 & $1.1 *$ \\
\hline 297 & 16439 & 1 & 1 & 21.0 & 14.0 & .6 & 2.2 & 4.6 & 1.8 & 6.7 & 1.6 \\
\hline 297 & 16439 & 1 & 2 & 12.3 & 11.9 & .8 & 2.1 & 1.5 & 1.3 & 3.4 & 1.2 \\
\hline 297 & 46439 & 2 & 1 & 22.3 & 9.1 & -1.4 & 1.8 & 3.9 & .9 & 1.0 & 1.0 \\
\hline 297 & 46439 & 2 & 2 & 46.1 & 14.6 & -1.1 & 1.6 & 1.0 & 1.1 & 4.3 & 1.3 \\
\hline 299 & 16732 & 1 & 1 & 16.1 & 8.8 & -.6 & 1.7 & 3.2 & 1.1 & 3.3 & 1.1 \\
\hline 299 & 46732 & 2 & $i$ & 15.9 & 12.9 & 1.8 & 1.9 & 2.1 & 1.6 & 4.6 & 1.3 \\
\hline 301 & 16986 & 1 & 1 & 21.0 & 9.0 & -1.0 & 1.7 & 1.7 & .8 & 3.8 & .9 \\
\hline 301 & 16996 & 1 & 2 & 26.6 & 12.9 & .2 & 1.9 & 2.3 & 1.4 & 5.2 & 1.4 \\
\hline 301 & 46986 & 7 & 1 & -6.1 & 8.1 & .2 & 1.5 & 1.7 & .9 & 3.5 & .9 \\
\hline 301 & 16986 & 7 & 2 & 14.9 & 9.8 & -.1 & 2.0 & 2.9 & .9 & 5.8 & 1.2 \\
\hline 301 & 16986 & 8 & 1 & -22.9 & 9.9 & 2.0 & 1.9 & 1.0 & 1.5 & 3.8 & 1.3 \\
\hline $30 t$ & 46986 & 8 & 2 & -22.1 & 7.4 & .8 & 1.9 & 1.0 & .9 & 2.1 & .9 \\
\hline
\end{tabular}




\begin{tabular}{|c|c|c|c|c|c|c|c|c|c|c|c|}
\hline \multirow{3}{*}{$\begin{array}{l}\text { STAKE } \\
\text { NO. } \\
301\end{array}$} & \multirow{3}{*}{$\begin{array}{l}\text { DISTARCE } \\
\text { METERS } \\
46986\end{array}$} & \multicolumn{2}{|c|}{ SAMFLE } & \multicolumn{2}{|c|}{ THOKIUK } & \multicolumn{2}{|c|}{ RADIUn } & \multicolumn{2}{|c|}{ LEAI } & \multicolumn{2}{|c|}{ UKANIUH } \\
\hline & & TYPE & REF. & LEVEL & ERROK & LEVEL & ERROR & LEVEL & EKR'OR & LEVEL & EKKOR \\
\hline & & 8 & 3 & -11.0 & 6.7 & 2.1 & 1.5 & .1 & .9 & .5 & .9 \\
\hline 303 & 47454 & 1 & 1 & 28.3 & 12.0 & 1.0 & 1.5 & 2.3 & 1.0 & 1.9 & 1.1 \\
\hline 303 & 47454 & 1 & 2 & -2.4 & 9.3 & .7 & 2.1 & 2.2 & 1.0 & 5.6 & 1.2 \\
\hline 303 & 47454 & 2 & 1 & 45.8 & 11.9 & -2.1 & 2.4 & 3.4 & 1.1 & 9.0 & 1.3 \\
\hline 305 & 47795 & 1 & 1 & 18.0 & 9.9 & 2.8 & 1.7 & .8 & 1.0 & 2.2 & 1.1 \\
\hline 305 & 47795 & 1 & 2 & 115.1 & 16.9 & -3.1 & 2.0 & 2.9 & 1.5 & 7.2 & 1.5 \\
\hline 305 & 47795 & 1 & 3 & 19.0 & 10.0 & & & 1.5 & 1.1 & 1.1 & $.9 \%$ \\
\hline 305 & 47795 & 1 & 4 & 21.0 & 14.0 & 6.9 & 1.7 & 1.0 & 1.3 & -1.3 & $1.1:$ \\
\hline 305 & 47795 & 2 & 1 & 11.1 & 9.7 & .1 & 1.6 & 1.6 & .8 & 2.5 & .9 \\
\hline 305 & 47795 & 2 & 2 & 8.9 & 15.0 & 3.5 & 1.6 & 2.9 & 1.6 & -.1 & $.9 *$ \\
\hline 305 & 47795 & 3 & 1 & 50.0 & 11.0 & 4.9 & 1.9 & 3.0 & 1.2 & .2 & $.9+$ \\
\hline 307 & & 1 & 1 & 26.6 & 14.0 & -1.9 & 1.0 & 1.9 & 1.5 & 5.5 & 1.3 \\
\hline 307 & & 1 & 2 & -4.8 & 9.9 & -1.7 & 2.2 & 4.1 & 1.1 & 7.4 & 1.4 \\
\hline 307 & & 2 & 1 & 53.1 & 13.3 & 6.0 & 1.9 & 2.7 & 1.3 & 1.3 & 1.3 \\
\hline 307 & & 2 & 2 & 9.9 & 10.2 & .7 & 1.9 & 1.4 & .9 & 3.2 & 1.0 \\
\hline 307 & & 2 & 3 & 22.3 & 9.4 & -.4 & 1.8 & 1.0 & .9 & 5.1 & 1.2 \\
\hline 307 & & 3 & 1 & 24.8 & 11.9 & -1.2 & 2.5 & 6.1 & 1.3 & .5 & $.5 *$ \\
\hline 309 & & 1 & 1 & 22.3 & B. 8 & .4 & 1.7 & 1.9 & .9 & 3.6 & 1.0 \\
\hline 309 & & 1 & 2 & 30.1 & 12.4 & 1.4 & 1.8 & .8 & 1.3 & 3.8 & 1.3 \\
\hline 309 & & 2 & 2 & 42.5 & 14.6 & -.8 & 2.1 & .4 & 1.4 & 5.5 & 1.5 \\
\hline 309 & & 3 & 1 & 14.2 & 15.2 & -6.7 & 2.5 & 8.2 & 1.9 & .8 & $.5 *$ \\
\hline 309 & & 5 & 1 & 42.5 & 13.7 & .6 & 2.0 & -.3 & 1.2 & 5.5 & 1.4 \\
\hline 311 & & 1 & 1 & 19.8 & 9.4 & 4.3 & 1.9 & 1.4 & .5 & 3.1 & 1.0 \\
\hline 311 & & 1 & 2 & 13.6 & 9.0 & 1.0 & 1.8 & 2.5 & 1.0 & 4.3 & .9 \\
\hline 311 & & 2 & 1 & 53.2 & 11.4 & .2 & 2.2 & 3.2 & 1.0 & 6.7 & 1.2 \\
\hline 311 & & 2 & 2 & 74.4 & 14.6 & -2.0 & 1.8 & 3.8 & 1.6 & 4.7 & 1.3 \\
\hline 311 & & 6 & 1 & -13.9 & 8.0 & & & 2.5 & 1.1 & 1.1 & $.9 *$ \\
\hline 311 & & 7 & 1 & 60.3 & 14.2 & 1.1 & 2.0 & .8 & 1.4 & 5.1 & 1.3 \\
\hline 311 & & 7 & 2 & 38.4 & 11.1 & 1.2 & 2.2 & 5.0 & 1.1 & 6.1 & 1.2 \\
\hline 311 & & 7 & 3 & -3.4 & 10.6 & .9 & 1.6 & .6 & 1.3 & 1.3 & 1.2 \\
\hline 311 & & 8 & 1 & 30.1 & 12.1 & 1.9 & 1.7 & 4.0 & 1.3 & 2.3 & 1.2 \\
\hline 311 & & 8 & 2 & 7.1 & 11.7 & 1.8 & 1.9 & 1.5 & 1.4 & 2.8 & 1.4 \\
\hline 313 & & 1 & 1 & 24.8 & 12.8 & -3.4 & 1.7 & 4.2 & 1.3 & 4.7 & 1.4 \\
\hline 313 & & 1 & 2 & 8.7 & 7.7 & -1.5 & 1.8 & 1.7 & .9 & 3.0 & 1.0 \\
\hline 313 & & 1 & 3 & 26.0 & 9.5 & .1 & 1.9 & 1.0 & 1.1 & 5.4 & 1.1 \\
\hline 315 & & 1 & 1 & 1.2 & 7.7 & & & 1.8 & 1.0 & .2 & $.7:$ \\
\hline 315 & & 1 & 2 & 18.0 & 11.0 & 3.5 & 1.5 & 1.9 & 1.4 & .2 & $.8 *$ \\
\hline 315 & & 2 & 1 & 3.5 & 12.0 & 1.7 & 1.8 & 1.9 & 1.2 & -.3 & $.9 *$ \\
\hline 315 & & 2 & 2 & 22.0 & 10.0 & 2.5 & 1.5 & 1.8 & 1.0 & .9 & $.9 *$ \\
\hline 317 & & 1 & 1 & 12.4 & 8.6 & .2 & 1.7 & .3 & .8 & 2.2 & .9 \\
\hline 317 & & 1 & 2 & 7.1 & 12.3 & -.2 & 1.5 & .8 & 1.2 & 2.2 & 1.2 \\
\hline
\end{tabular}




\begin{tabular}{|c|c|c|c|c|c|c|c|c|c|c|c|}
\hline STAKE & DISTANCE & 5AH & OLE & THO & & & & & & UKA & \\
\hline NO. & HETERS & TYPE & REP. & LEUEL & ERRDK & LEVEL & ERROR & LEVEL & ERROK & I.EUEL & ERROR \\
\hline 317 & & 4 & 1 & -5.0 & 14.0 & 6.1 & 1.8 & 3.3 & 1.8 & 2.5 & $1.3 \%$ \\
\hline 317 & & 5 & 1 & 7.4 & 6.6 & 1.5 & 1.5 & 2.6 & .8 & 2.3 & .9 \\
\hline 319 & & 1 & 1 & 42.5 & 17.2 & -2.4 & 2.4 & 5.2 & 1.8 & 8.3 & 1.6 \\
\hline 319 & & 1 & 2 & 6.2 & 7.9 & -.0 & 1.6 & 2.2 & 1.1 & 3.2 & 1.0 \\
\hline 319 & & 2 & $t$ & 7.1 & 11.5 & -2.5 & 1.7 & 1.5 & 1.4 & 5.1 & 1.3 \\
\hline 319 & & 2 & 2 & 17.3 & 10.1 & .7 & 1.8 & 1.7 & 1.0 & 4.3 & 1.1 \\
\hline 321 & & 1 & 1 & .0 & 6.6 & 2.5 & 1.6 & .7 &.$\theta$ & 1.3 & .8 \\
\hline 321 & & 1 & 2 & 40.7 & 11.3 & -.2 & 1.9 & 1.9 & 1.5 & 4.5 & 1.3 \\
\hline 321 & & 4 & 1 & 26.0 & 16.0 & 4.0 & 1.7 & 5.6 & 2.0 & -1.1 & 1.04 \\
\hline 321 & & 6 & 1 & -1.9 & 11.9 & -.8 & 1.9 & 2.5 & 1.5 & 5.1 & $\$ .4$ \\
\hline 321 & & 8 & 1 & -3.6 & 8.3 & 1.3 & 1.6 & 1.2 &.$\theta$ & 2.2 & .9 \\
\hline 321 & & 8 & 2 & 6.2 & 9.5 & .9 & 2.0 & 1.9 & 1.0 & 5.3 & 1.2 \\
\hline 323 & & 1 & 1 & 35.4 & 13.2 & -1.4 & 1.5 & .0 & 1.4 & 3.0 & 1.2 \\
\hline 323 & & 1 & 2 & 53.1 & 14.2 & .2 & 2.1 & 4.6 & 1.1 & 7.7 & 1.4 \\
\hline 323 & & 2 & 1 & 26.0 & 10.0 & -.6 & 1.7 & $2 . \theta$ & 1.0 & 4.4 & 1.2 \\
\hline 323 & & 9 & 1 & 76.2 & 16.7 & -.1 & 2.1 & 4.8 & 1.7 & 8.3 & 1.6 \\
\hline 325 & & 1 & 1 & 33.0 & 11.0 & & & 1.9 & 1.1 & 1.1 & $1.0 *$ \\
\hline 325 & & 2 & 1 & .0 & 12.0 & & & 3.6 & 1.5 & 2.0 & $1.2 *$ \\
\hline 327 & & 1 & 1 & 31.0 & 9.3 & -3.2 & 1.6 & 1.0 & .9 & 4.5 & 1.0 \\
\hline 327 & & 1 & 2 & 60.3 & 14.6 & 2.8 & 1.8 & 2.1 & 1.3 & 3.4 & 1.4 \\
\hline 327 & & 2 & 1 & 92.1 & 17.4 & -2.0 & 2.3 & 2.3 & 1.8 & 11.6 & 1.7 \\
\hline 329 & & 1 & 1 & 14.9 & .9 & 1.8 & 1.6 & 2.2 & .9 & 3.2 & 1.0 \\
\hline 329 & & 1 & 2 & 37.2 & 11.1 & .5 & 1.9 & 2.3 & -1.0 & 4.8 & 1.1 \\
\hline 331 & & 1 & 1 & 24.8 & 13.5 & -.7 & 1.9 & 3.1 & 1.5 & 4.6 & 1.4 \\
\hline 331 & & 4 & 1 & 26.6 & 11.9 & -.8 & 1.8 & 1.1 & .9 & 3.4 & 1.1 \\
\hline 331 & & 4 & 2 & 64.0 & 16.0 & 3.7 & 1.8 & 3.3 & 1.8 & 2.3 & $1.2 *$ \\
\hline 331 & & 6 & 1 & 14.0 & 7.7 & & & 1.5 & 1.0 & -.3 & 1.0 \\
\hline 331 & & 8 & 1 & 7.4 & 9.2 & -.4 & 1.6 & $\$ .2$ & 1.0 & 2.4 & 1.0 \\
\hline 331 & & 8 & 2 & -1.7 & 9.8 & 3.0 & 1.7 & 2.5 & 1.1 & 3.2 & 1.1 \\
\hline 331 & & 8 & 3 & -12.3 & B. 0 & 1.6 & 2.1 & 2.2 & .9 & 4.9 & 1.2 \\
\hline 333 & & 1 & 1 & 37.2 & 14.7 & -.6 & 1.8 & 2.9 & 1.4 & 4.8 & 1.3 \\
\hline 333 & & 1 & 2 & .0 & 8.4 & 2.6 & 1.8 & 1.0 & .8 & 1.9 & 1.0 \\
\hline 333 & & 2 & 1 & 21.3 & 12.8 & 1.3 & 1.5 & 3.3 & 1.5 & 2.3 & 1.2 \\
\hline 333 & & 4 & 1 & 63.7 & 17.2 & -1.2 & 2.5 & 5.8 & 2.0 & 7.9 & 1.7 \\
\hline 335 & & 1 & 1 & 22.3 & 9.3 & .0 & 2.0 & 1.9 & 1.1 & 4. 8 & 1.1 \\
\hline 335 & & 1 & 2 & 19.4 & 13.1 & -.2 & 1.9 & 2.9 & 1.4 & 5.5 & 1.4 \\
\hline 335 & & 2 & 1 & B. 7 & 8.5 & 1.1 & 1.9 & .6 & .9 & 3.0 & 1.0 \\
\hline 335 & & 2 & 2 & 13.6 & B. 9 & .0 & 1.7 & 2.8 & 1.1 & 3.0 & 1.0 \\
\hline 335 & & 4 & 1 & 134.9 & 17.7 & -2.1 & 2.9 & 13.9 & 1.8 & 14.8 & 1.8 \\
\hline 337 & & 1 & 1 & $1 \uparrow 1.6$ & 16.3 & 1.1 & 2.3 & 5.6 & 1.5 & 8.5 & 1.5 \\
\hline 337 & & $i$ & 2 & 31.9 & 14.6 & -1.8 & 1.8 & 2.9 & 1.5 & 5.3 & 1.4 \\
\hline
\end{tabular}




\begin{tabular}{|c|c|c|c|c|c|c|c|c|c|c|c|}
\hline STAKE & DISTANCE & SAMI & & THO & & & & & & & \\
\hline NO. & METERS & TYPE & REP. & LEVEL & ERROR & LEVEL & ERROR & LEVEL & ERROR & LEVEL & ERROR \\
\hline 337 & & 2 & 1 & 42.5 & 12.3 & .3 & 2.2 & 4.0 & 1.5 & 5.0 & 1.2 \\
\hline 337 & & 3 & 1 & 14.8 & 9.9 & -.7 & 1.8 & 1.5 & 1.0 & 3.0 & 1.0 \\
\hline 339 & & 1 & 1 & 17.7 & 14.4 & -.6 & 2.2 & 5.9 & 1.7 & 5.7 & 1.5 \\
\hline 339 & & 1 & 2 & 48.3 & 13.5 & 1.8 & 2.3 & 7.7 & 1.5 & 6.4 & 1.4 \\
\hline 339 & & 1 & 3 & 40.7 & 14.3 & -3.3 & 2.1 & 1.0 & 1.3 & 5.8 & 1.5 \\
\hline 339 & & 2 & 1 & 125.8 & 18.5 & -2.1 & 2.4 & 6.9 & 1.8 & 10.9 & 1.7 \\
\hline 341 & & 1 & 1 & 14.2 & 12.3 & -.5 & 1.8 & 2.1 & 1.4 & 4.3 & 1.3 \\
\hline 341 & & 1 & 2 & 34.7 & 10.1 & -.9 & 1.8 & 2.5 & 1.0 & 4.7 & 1.1 \\
\hline 341 & & 2 & 1 & 22.3 & B.9 & -.1 & 1.7 & 2.3 & 1.0 & 3.7 & 1.1 \\
\hline 341 & & 2 & 2 & 1.8 & 12.3 & .2 & 2.2 & 2.9 & 1.5 & 6.0 & 1.5 \\
\hline 341 & & 2 & 3 & 23.0 & 14.3 & -.6 & 1.9 & 1.9 & 1.5 & 5.6 & 1.5 \\
\hline 341 & & 4 & 1 & 11.1 & 10.0 & 1.4 & 2.3 & 2.9 & 1.0 & 5.0 & 1.2 \\
\hline 341 & & 4 & 2 & 102.7 & 20.8 & 3.3 & 3.0 & 14.8 & 2.6 & 13.7 & 2.0 \\
\hline 341 & & 4 & 3 & 40.7 & 13.6 & -3.8 & 2.3 & 5.4 & 1.6 & 12.4 & 1.6 \\
\hline 341 & & 5 & 1 & 42.5 & 15.6 & .5 & 2.2 & 2.7 & 1.6 & 7.2 & 9.6 \\
\hline $34:$ & & 6 & 1 & 3.5 & 10.9 & .0 & 1.8 & 2.3 & 1.5 & 4.2 & 1.3 \\
\hline 341 & & 6 & 2 & 9.9 & 10.0 & 1.1 & 2.1 & 2.3 & .9 & 4.5 & 1.2 \\
\hline 341 & & 7 & $\$$ & .0 & 9.6 & -.4 & 2.0 & 1.8 & 1.0 & 4.1 & 1.1 \\
\hline 341 & & 7 & 2 & 6.2 & 7.7 & 1.8 & 1.3 & 2.3 & .9 & 1.8 & .8 \\
\hline 341 & & 7 & 3 & 11.1 & 9.0 & .5 & 1.6 & .4 & .9 & 3.8 & 1.0 \\
\hline 341 & & 8 & 1 & 31.9 & 13.0 & 3.0 & 2.3 & 4.4 & 1.5 & 4.8 & 1.3 \\
\hline 341 & & B & 2 & 18.6 & 9.8 & 4.9 & 2.1 & 1.8 & 1.2 & 4.4 & 1.1 \\
\hline 343 & & 1 & 1 & 23.0 & 12.4 & .0 & 2.0 & 1.0 & 1.3 & 4.6 & 1.4 \\
\hline 343 & & 1 & 2 & -7.0 & 10.9 & -.0 & 2.4 & 1.9 & 1.4 & 9.1 & 1.4 \\
\hline 343 & & 3 & 1 & 125.7 & 20.6 & -.9 & 3.1 & 8.3 & 2.2 & 14.1 & 2.0 \\
\hline 343 & & 3 & 2 & 115.1 & 17.5 & 1.7 & 2.6 & 13.2 & 1.8 & 11.6 & 1.7 \\
\hline 343 & & 9 & 1 & 12.3 & 11.6 & 2.3 & 1.8 & 3.1 & 1.1 & 4.7 & 1.3 \\
\hline 345 & & 1 & 1 & 8.7 & 8.7 & -1.0 & 2.0 & 2.2 & 1.0 & 5.1 & 1.2 \\
\hline 345 & & 1 & 2 & 5.3 & 12.4 & -2.4 & 2.0 & 1.9 & 1.5 & 7.7 & 1.4 \\
\hline 345 & & 2 & 1 & 18.6 & 8.3 & -1.6 & 1.8 & 2.9 & 1.0 & 3.9 & .9 \\
\hline 345 & & 3 & 1 & 92.1 & 19.1 & -.5 & 2.1 & 6.1 & 1.7 & 7.6 & 1.7 \\
\hline 347 & & 1 & 1 & 7.4 & 7.6 & 2.4 & 1.5 & 1.6 & .9 & 2.5 & 1.0 \\
\hline 347 & & 1 & 2 & 46.0 & 11.7 & -1.0 & 2.0 & -.9 & 1.3 & 5.0 & 1.2 \\
\hline 347 & & 2 & 1 & 23.0 & 16.7 & 3.4 & 2.2 & 7.1 & 1.9 & 5.0 & 1.8 \\
\hline 347 & & 3 & 1 & 5.0 & 8.7 & .1 & 1.5 & 2.5 & 1.0 & 2.9 & 1.1 \\
\hline 347 & & 3 & 2 & 126.3 & 17.5 & 2.4 & 3.2 & 13.5 & 1.6 & 14.2 & 1.7 \\
\hline 349 & & 1 & 1 & 69.1 & 16.9 & -1.6 & 2.4 & 3.3 & 1.6 & 9.6 & 1.6 \\
\hline 349 & & 1 & 2 & 21.0 & 9.5 & -.1 & 1.8 & 3.2 & 1.0 & 5.1 & 1.1 \\
\hline 349 & & 2 & 1 & 17.3 & 8.0 & -.5 & 1.9 & 1.8 & 1.0 & 5.9 & 1.4 \\
\hline 349 & & 3 & 1 & 87.9 & 15.7 & -3.3 & 2.3 & 6.3 & 1.5 & 10.5 & 1.5 \\
\hline 351 & & 1 & 1 & 2.5 & 7.3 & -1.3 & 1.8 & 1.9 & .9 & 3.5 & 1.0 \\
\hline
\end{tabular}




\begin{tabular}{|c|c|c|c|c|c|c|c|c|c|c|c|}
\hline STAKE & DISTANCE & SAHI & PLE & Тно & & & & & & URA & \\
\hline No. & HETERS & TYPE & REP. & LEVEL & ERROR & LEVEL & ERROR & LEVEL & ERROR & LEVEL & ERRBR \\
\hline 351 & & 1 & 2 & 70.9 & 16.4 & -3.7 & 2.1 & 3.8 & 1.0 & 6.5 & 1.5 \\
\hline 351 & & 2 & 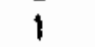 & 8.9 & 11.6 & 2.4 & 1.9 & .4 & 1.1 & 2.0 & 1.3 \\
\hline 351 & & 2 & 2 & 7.4 & 8.9 & 1.5 & 1.9 & 3.3 & 1.0 & 3.5 & 1.0 \\
\hline 351 & & 3 & 1 & 111.4 & 17.2 & -1.9 & 2.9 & 12.0 & 1.9 & 12.4 & 1.8 \\
\hline 351 & & 6 & 1 & 7.1 & 10.6 & 2.7 & 1.9 & 1.7 & 1.5 & 5.7 & 1.4 \\
\hline 351 & & 6 & 2 & 7.4 & 9.0 & -.4 & 1.7 & 1.0 & 1.1 & 2.7 & 1.1 \\
\hline 351 & & 7 & 1 & 23.5 & 9.0 & 1.0 & 1.7 & 2.2 & 1.1 & 2.9 & 1.0 \\
\hline 351 & & 8 & 1 & 33.1 & 13.7 & .7 & 2.1 & 2.7 & 1.7 & 7.3 & 1.5 \\
\hline 351 & & 8 & 2 & 19.4 & 10.5 & -.5 & 1.9 & 1.7 & 1.2 & 4.7 & 1.2 \\
\hline 351 & & 1 & 3 & 8.7 & 10.4 & -2.2 & 2.3 & 3.3 & 1.1 & 11.1 & 1.3 \\
\hline 353 & & 1 & 1 & 8.9 & 11.6 & 1.8 & 1.8 & 3.1 & 1.4 & 3.7 & 1.2 \\
\hline 353 & & 1 & 2 & .0 & 8.4 & 2.0 & 1.5 & 1.8 & .9 & 2.0 & 1.0 \\
\hline 353 & & 2 & 1 & 7.4 & 9.9 & -1.9 & 2.0 & 3.0 & 1.1 & 4.6 & 1.2 \\
\hline 353 & & 2 & 2 & 3.5 & 13.0 & -.4 & 2.3 & .0 & 1.4 & 5.0 & 1.4 \\
\hline 353 & & 3 & $\$$ & 131.1 & 22.4 & 5.9 & 2.9 & 9.8 & 2.5 & 5.7 & 2.0 \\
\hline 355 & & 1 & 1 & 32.0 & 13.0 & & & 3.1 & 1.6 & .2 & $1.0 *$ \\
\hline 355 & & 1 & 2 & -2.3 & 8.7 & & & 1.2 & .9 & -.6 & $.9 *$ \\
\hline 355 & & 2 & 1 & -7.3 & 9.0 & & & 3.6 & 1.1 & .4 & $.8 *$ \\
\hline 355 & & 2 & 2 & 25.0 & 13.0 & & & 2.3 & 1.3 & 2.0 & $1.1 *$ \\
\hline 355 & & 3 & 1 & 21.0 & 9.7 & & & 1.6 & 1.0 & -1.5 & $1.1 *$ \\
\hline 357 & & 1 & 1 & 7.1 & 10.6 & 1.0 & 1.6 & 1.9 & 1.2 & 3.2 & 1.1 \\
\hline 357 & & 2 & 1 & 23.0 & 11.6 & -1.5 & 1.7 & 1.3 & 1.4 & 5.3 & 1.4 \\
\hline 357 & & 2 & 2 & 47.8 & 16.5 & 4.8 & 2.3 & 4.4 & 1.7 & 4.3 & 1.5 \\
\hline 357 & & 3 & 1 & 23.5 & 9.5 & -6.4 & 2.1 & 1.0 & 1.1 & .6 & $.3 *$ \\
\hline 359 & & 1 & $\uparrow$ & 23.5 & 8.1 & -.4 & 1.7 & 1.2 & .9 & 3.7 & 1.0 \\
\hline 359 & & 1 & 2 & 14.2 & 13.3 & -1.6 & 2.2 & 3.8 & 1.6 & 7.8 & 1.6 \\
\hline 359 & & 2 & 1 & -3.2 & 10.0 & -1.2 & 1.9 & 1.7 & 9.2 & 4.5 & 1.4 \\
\hline 359 & & 2 & 2 & 39.6 & 10.7 & .8 & 2.1 & 2.9 & 1.1 & 4.9 & 1.1 \\
\hline 359 & & 3 & 9 & 107.7 & 19.0 & -20.3 & 3.7 & 14.1 & 1.9 & 2.0 & $.6 *$ \\
\hline 361 & & 1 & 1 & 24.7 & 9.4 & .9 & 2.0 & 1.8 & 1.1 & 3.0 & 1.1 \\
\hline 361 & & 1 & 2 & 23.0 & 11.3 & -.0 & 2.1 & 1.9 & 1.2 & 4.3 & 1.2 \\
\hline 361 & & 2 & 1 & 19.8 & 10.5 & 1.7 & 1.6 & 4.7 & 1.1 & 2.7 & 1.0 \\
\hline 361 & & 2 & 2 & 9.9 & 8.9 & -1.3 & 1.9 & 3.2 & 1.0 & 3.8 & $\uparrow .1$ \\
\hline 361 & & 3 & 1 & 1.8 & 12.9 & 0.1 & 1.2 & .6 & 1.4 & -.2 & $.6 \%$ \\
\hline 361 & & $b$ & 2 & -1.1 & 7.1 & -.4 & 1.0 & 1.2 & 1.0 & .2 & $.4 *$ \\
\hline 361 & & 7 & 1 & -4.9 & 7.4 & .1 & 1.5 & 1.5 & .9 & 2.7 & 1.0 \\
\hline 361 & & 7 & 2 & 10.6 & 11.2 & 2.1 & 1.9 & 2.1 & 1.3 & 3.7 & 1.4 \\
\hline 361 & & 8 & 1 & -17.6 & 10.0 & .2 & 1.7 & 1.3 & 1.2 & 1.0 & 1.1 \\
\hline 363 & & 1 & 1 & 3.7 & 9.4 & .7 & 1.8 & 1.2 & 1.0 & 3.9 & 1.1 \\
\hline 363 & & 1 & 2 & 6.2 & 8. 3 & -.0 & 1.7 & 3.3 & .7 & 2.9 & 1.0 \\
\hline 363 & & 2 & 1 & 31.9 & 13.3 & .8 & 1.7 & 1.3 & 1.4 & 3.1 & 1.4 \\
\hline
\end{tabular}




\begin{tabular}{|c|c|c|c|c|c|c|c|c|c|c|c|}
\hline STAKE & DISTANCE & SAHF & LEE & THO & & & & & & URA & \\
\hline NO. & METERS & TYPE & REP. & LEVEL & ERROR & LEVEL & ERROK & LEVEL & ERROR & LEVEL & ERROK \\
\hline 363 & & 2 & 2 & 19.5 & 11.9 & 3.0 & 1.7 & 1.5 & 1.3 & 1.4 & 1.3 \\
\hline 363 & & 3 & 1 & 39.6 & 13.2 & 20.2 & .1 & 2.3 & 1.1 & -.7 & $.5 *$ \\
\hline 365 & & 1 & 1 & 18.0 & 14.0 & 1.2 & 1.7 & 3.8 & 1.5 & .8 & $1.0 *$ \\
\hline 365 & & 1 & 2 & -2.4 & 9.4 & 7.9 & 1.8 & 1.7 & 1.0 & -1.0 & $.8 *$ \\
\hline 365 & & 2 & 1 & 51.0 & 11.0 & 2.2 & 1.6 & 4.3 & 1.2 & -.1 & $1.1 *$ \\
\hline 365 & & 2 & 2 & 26.0 & 10.0 & & & 2.5 & .9 & .9 & $.9: *$ \\
\hline 365 & & 3 & 1 & 44.5 & 11.2 & 1.2 & 2.1 & 2.3 & 1.1 & .2 & $.4 *$ \\
\hline 367 & & 1 & 1 & 9.9 & 9,1 & -.2 & 1.9 & 4.3 & 1.1 & 4.9 & 1.1 \\
\hline 367 & & 1 & 2 & 1.2 & 7.9 & -.2 & 2.2 & .0 & 1.0 & 5.2 & 1.2 \\
\hline 367 & & 3 & 1 & 118.7 & 20.1 & .8 & 3.1 & 13.8 & 2.2 & .9 & $B:$ \\
\hline 369 & & 1 & 1 & 12.4 & 10.8 & .8 & 1.5 & 2.1 & 1.3 & .1 & 1.2 \\
\hline 369 & & 1 & 2 & 21.3 & 12.3 & -.9 & 1.7 & 1.0 & 1.3 & 3.6 & 1.3 \\
\hline 369 & & 3 & 1 & 33.0 & 10.0 & & & .4 & 1.0 & -.4 & .9 \\
\hline 369 & & 4 & 1 & 12.0 & 10.0 & 1.2 & 1.2 & 2.3 & 1.4 & .4 & .8 \\
\hline 369 & & 5 & 1 & 24.0 & 12.0 & 6.3 & 2.0 & 5.5 & 1.4 & -.6 & 1.1 \\
\hline 371 & & 1 & 1 & 3.5 & 13.7 & -1.4 & 2.0 & .4 & 1.5 & 6.9 & 1.6 \\
\hline 371 & & 1 & 2 & 15.9 & 12.7 & -.1 & 1.8 & 1.1 & 1.0 & 4.0 & 1.3 \\
\hline 371 & & 2 & 1 & 37.1 & 10.7 & -.5 & 1.6 & 4.3 & 1.0 & 3.4 & 1.1 \\
\hline 371 & & 2 & 2 & 26.6 & 12.5 & -.6 & 1.6 & 1.5 & 1.2 & 4.3 & 1.2 \\
\hline 371 & & 3 & 1 & 19.8 & 7.8 & 5.5 & 2.0 & 1.0 & .9 & 3.4 & .9 \\
\hline 371 & & 6 & 1 & 0.7 & 9.0 & 1.3 & 2.0 & .7 & 1.0 & 4.2 & 1.0 \\
\hline 371 & & 6 & 2 & 1.2 & 7.9 & $=.0$ & 1.9 & .6 & 1.0 & 3.2 & 1.0 \\
\hline 371 & & 7 & 1 & 8.7 & 7.1 & .1 & 1.4 & 1.2 & .8 & 2.3 & .9 \\
\hline 371 & & 7 & 2 & -2.4 & 7.4 & .0 & 1.6 & 1.1 & .8 & 3.1 & .9 \\
\hline 371 & & 8 & 1 & 28.3 & 11.5 & 1.9 & 1.5 & -.9 & 1.2 & 1.0 & 1.0 \\
\hline 371 & & 8 & 2 & 10.6 & 10.6 & -1.0 & 1.8 & 1.9 & 1.2 & 4.0 & 1.2 \\
\hline 373 & & 1 & 1 & 35.9 & 10.4 & 2.9 & 2.1 & 2.5 & .9 & 4.9 & 1.1 \\
\hline 373 & & 1 & 2 & 40.7 & 13.4 & -.6 & 2.0 & 1.9 & 1.4 & 6.1 & 1.4 \\
\hline 373 & & 2 & $\uparrow$ & -1.7 & 14.7 & 3.0 & 2.0 & 1.1 & 1.3 & 4.0 & 1.4 \\
\hline 373 & & 2 & 2 & 24.8 & 9.8 & -1.5 & 2.0 & 2.3 & 1.0 & 5.5 & 1.1 \\
\hline 373 & & 3 & 1 & 45.8 & 12.2 & .4 & 2.1 & 5.0 & 1.1 & 7.1 & 1.2 \\
\hline 373 & & 4 & 1 & 141.7 & 22.9 & -.5 & 2.8 & 14.0 & 2.5 & 13.0 & 2.0 \\
\hline 373 & & 5 & 1 & 62.0 & 17.6 & 2.5 & 2.5 & 8.4 & 1.9 & 6.5 & 1.6 \\
\hline 375 & & 1 & 1 & 3.7 & 9.0 & -.8 & 1.8 & 7.2 & .9 & 3.2 & 1.1 \\
\hline 375 & & 2 & 1 & 16.0 & 8.9 & -1.2 & 1.6 & 1.5 & .9 & 3.0 & 1.0 \\
\hline 375 & & 3 & 1 & 33.3 & 10.1 & .2 & 1.9 & 5.1 & 1.1 & 4.9 & 1.1 \\
\hline 375 & & 9 & 1 & 12.4 & 10.0 & .5 & 3.2 & 3.7 & 1.0 & 18.3 & 1.7 \\
\hline 377 & & 1 & 1 & 14.9 & 8.8 & .6 & 2.0 & 3.5 & 1.1 & 4.9 & 1.1 \\
\hline 377 & & 1 & 2 & 46.1 & 14.2 & -1.6 & 2.4 & 7.7 & 1.8 & 7.5 & 1.6 \\
\hline 377 & & 2 & 1 & 14.9 & 8.6 & 1.7 & 1.8 & 2.0 & .8 & 3.4 & 1.0 \\
\hline 377 & & 3 & 1 & 47.0 & 12.1 & .8 & 2.3 & 3.2 & 1.2 & 9.0 & 1.3 \\
\hline
\end{tabular}




\begin{tabular}{|c|c|c|c|c|c|c|c|c|c|c|c|}
\hline STAKE & DISTANCE & SAY & PLE & THC & & & & & & URA & \\
\hline No. & HETERS & TYPE & REF. & LEVEL & ERROR & LEVEL & ERRDR & LEVEL & ERROR & LEUEL & ERROR \\
\hline 379 & & 1 & 1 & 31.9 & 14.0 & .6 & 2.1 & 2.9 & 1.6 & 3.3 & 1.5 \\
\hline 379 & & 1 & 2 & 11.1 & 9.2 & 1.6 & 1.7 & 1.2 & 1.1 & 3.5 & 1.0 \\
\hline 379 & & 2 & 1 & -2.4 & 9.4 & 3.5 & 2.1 & .4 & 1.0 & 1.1 & 1.0 \\
\hline 379 & & 3 & 1 & 10.6 & 11.8 & .9 & 1.8 & -.3 & 1.2 & 3.8 & 1.1 \\
\hline 381 & & 1 & 1 & 28.3 & 14.3 & -1.2 & 1.9 & 1.0 & 1.4 & 4.8 & 1.4 \\
\hline 381 & & 1 & 2 & 31.9 & 13.5 & -.9 & 1.9 & 2.3 & 1.6 & 4.8 & 1.4 \\
\hline 381 & & 2 & 1 & 12.4 & 11.3 & .3 & 1.8 & 4.0 & 1.4 & 2.5 & 1.2 \\
\hline 381 & & 3 & 1 & 7.1 & 10.9 & 1.8 & 1.8 & 2.3 & 1.2 & 4.6 & 1.2 \\
\hline 381 & & 6 & 1 & 8.7 & 10.1 & 1.0 & 2.0 & 1.8 & 1.1 & 3.2 & 1.1 \\
\hline 381 & & 6 & 2 & -4.9 & 10.2 & 5.5 & 1.7 & .7 & 1.0 & 1.7 & 1.1 \\
\hline 381 & & 7 & 1 & 15.9 & 13.6 & -2.3 & 1.9 & 2.1 & 1.2 & 5.6 & 1.5 \\
\hline 381 & & 7 & 2 & 18.6 & 8.8 & -3.8 & 1.8 & 1.8 & 1.0 & 4.2 & 1.0 \\
\hline 381 & & 7 & 3 & 5.3 & 9.5 & .2 & 1.7 & -.5 & 1.2 & 2.7 & 1.1 \\
\hline 381 & & 8 & 1 & -6.1 & 8.3 & 2.2 & 1.7 & 1.7 & .9 & 1.3 & 1.0 \\
\hline 381 & & 8 & 2 & 5.3 & 11.3 & 1.1 & 1.7 & 2.9 & 1.5 & 1.8 & 1.1 \\
\hline 383 & & 1 & 1 & 53.2 & 12.2 & .3 & 2.3 & 2.8 & 1.1 & 7.2 & 1.2 \\
\hline 383 & & 1 & 2 & 26.6 & 11.6 & 1.0 & 2.1 & 4.2 & 1.5 & 5.3 & 1.3 \\
\hline 383 & & 1 & 3 & 17.7 & 12.5 & 1.5 & 1.7 & .4 & 1.4 & 3.3 & 1.2 \\
\hline 383 & & 3 & 1 & 19.5 & 13.6 & 2.8 & 2.0 & 1.3 & 1.4 & 4.3 & 1.4 \\
\hline 385 & & 1 & 1 & -7.0 & 11.2 & .4 & 2.2 & 1.1 & 1.4 & 5.7 & 1.5 \\
\hline 385 & & 1 & 2 & 19.8 & 9.3 & .2 & 2.0 & 2.9 & 1.0 & 4.2 & 1.1 \\
\hline 385 & & 2 & 1 & 1.8 & 12.4 & 1.8 & 2.2 & 2.3 & 1.4 & 2.9 & 1.4 \\
\hline 385 & & 3 & 1 & 74.3 & 14.9 & -.7 & 2.6 & 7.9 & 1.5 & 12.4 & 1.6 \\
\hline 385 & & 4 & 1 & -8.8 & 14.7 & .0 & 2.2 & 5.6 & 1.7 & 5.5 & 1.6 \\
\hline 385 & & 5 & 1 & .0 & 9.8 & 1.3 & 1.0 & 2.3 & 1.1 & 3.9 & 1.0 \\
\hline 387 & & 1 & 1 & 54.9 & 14.5 & -.8 & 2.1 & 1.5 & 1.6 & 7.6 & 1.5 \\
\hline 387 & & 2 & 1 & -4.9 & 6.8 & 2.0 & 1.4 & -.2 & .8 & 2.3 & .8 \\
\hline 387 & & 3 & 1 & 99.2 & 18.1 & -1.1 & 2.5 & 0.7 & 1.8 & 9.3 & 1.7 \\
\hline 387 & & 4 & 1 & 10.8 & 20.0 & .9 & 2.6 & 11.3 & 2.3 & 10.0 & 2.0 \\
\hline 387 & & 5 & 1 & 17.0 & 11.1 & 2.8 & 2.1 & 4.4 & 1.0 & 4.8 & 1.2 \\
\hline 389 & & 1 & 1 & 1.7 & 10.5 & 1.2 & 1.8 & -1.8 & 1.3 & 3.3 & 1.3 \\
\hline 389 & & 1 & 2 & 17.3 & 9.3 & -2.1 & 1.9 & 2.8 & 1.0 & 4.1 & 1.1 \\
\hline 389 & & 2 & 1 & 5.0 & 11.2 & -2.3 & 2.3 & 4.3 & 1.3 & 4.8 & 1.3 \\
\hline 389 & & 3 & 1 & 93.9 & 19.3 & -.0 & 2.6 & 5.2 & 2.0 & 9.4 & 1.7 \\
\hline 389 & & 9 & 1 & 47.0 & 11.4 & 2.7 & 3.8 & 2.6 & 1.0 & 27.6 & 1.9 \\
\hline 391 & & 1 & 1 & -1.1 & 8.8 & .6 & 2.0 & .3 & 1.0 & 4.1 & 1.2 \\
\hline 391 & & 3 & 1 & 138.7 & 22.7 & 6.4 & 2.9 & 16.1 & 2.4 & 9.7 & 2.0 \\
\hline 391 & & 6 & 1 & -14.2 & 12.3 & .2 & 2.1 & .2 & 1.5 & 6.1 & 1.4 \\
\hline 391 & & 6 & 2 & 9.9 & 9.9 & 1.1 & 2.0 & 3.7 & 1.2 & 3.1 & 1.2 \\
\hline 391 & & 7 & 1 & 47.0 & 11.3 & 3.4 & 2.1 & 1.4 & 1.0 & 6.1 & 1.2 \\
\hline 391 & & 7 & 2 & 17.3 & 8.2 & 1.6 & 1.9 & .8 & .9 & 4.5 & 1.0 \\
\hline
\end{tabular}




\begin{tabular}{|c|c|c|c|c|c|c|c|c|c|c|c|}
\hline \multirow{3}{*}{$\begin{array}{l}\text { STAKE } \\
\text { ND. } \\
391\end{array}$} & \multirow{2}{*}{$\begin{array}{l}\text { DISTANCE } \\
\text { HETERS }\end{array}$} & \multicolumn{2}{|c|}{ SAHPLE } & \multicolumn{2}{|c|}{ THOR IUH } & \multicolumn{2}{|c|}{ RABIUn } & \multicolumn{2}{|c|}{ LEAD } & \multicolumn{2}{|c|}{ URANIUH } \\
\hline & & TYPE & REP. & LEVEL & ERROR & LEVEL & ERROR & LEVEL & ERROR & LEVEL & ERROR \\
\hline & & 8 & 1 & -12.2 & 9.2 & 2.7 & 1.5 & 1.0 & 1.0 & .3 & 1.2 \\
\hline 391 & & 8 & 2 & 3.5 & 12.3 & 1.2 & 2.4 & .0 & 1.4 & 6.2 & 1.4 \\
\hline 393 & & 1 & 1 & 10.6 & 12.3 & -.3 & 1.9 & 1.9 & 1.3 & 5.2 & 1.4 \\
\hline 393 & & 1 & 2 & .0 & 8.6 & -2.5 & 1.8 & 1.8 & .9 & 5.7 & 1.1 \\
\hline 393 & & 2 & 1 & 14.9 & 8.9 & -.9 & 1.4 & 1.8 & .9 & 2.4 & 1.0 \\
\hline 393 & & 4 & 1 & 73.0 & 13.0 & 12.8 & 1.5 & 5.8 & 1.2 & -.2 & $.5 *$ \\
\hline 395 & & 1 & 1 & .0 & 13.0 & & & 5.0 & 1.6 & 1.2 & $1.0 *$ \\
\hline 395 & & 1 & 2 & 30.0 & 10.0 & & & 1.1 & 1.1 & 2.7 & $1.2 *$ \\
\hline 395 & & 2 & 1 & B. 8 & 14.0 & & & .4 & 1.6 & -1.1 & $1.0=$ \\
\hline 395 & & 4 & 1 & 39.0 & 12.0 & & & 3.3 & 1.7 & 2.0 & $1.1 *$ \\
\hline 395 & & 4 & 2 & 21.0 & 9.5 & -10.9 & 2.5 & 1.9 & 1.0 & .9 & $.4 *$ \\
\hline 395 & & 4 & 3 & 47.0 & 12.0 & & & 5.8 & 1.3 & .5 & $.9 *$ \\
\hline 397 & & 1 & 1 & 23.5 & 9.7 & -.6 & 1.9 & 2.9 & .9 & 4.3 & 1.1 \\
\hline 397 & & 1 & 2 & 23.5 & 10.0 & .6 & 2.2 & 2.5 & 1.0 & 5.1 & 1.1 \\
\hline 397 & & 4 & 1 & 30.1 & 14.7 & 7.9 & 2.1 & 4.4 & 1.5 & -.8 & $.5 *$ \\
\hline 399 & & 1 & 1 & 7.4 & 8.8 & 2.6 & 1.7 & 3.9 & .9 & 2.3 & $1.0 *$ \\
\hline 399 & & 2 & 1 & 3.5 & 10.3 & .5 & 1.6 & 4.2 & 1.5 & 2.2 & 1.3 \\
\hline 399 & & 4 & 1 & 14.9 & B. 8 & 5.2 & 1.6 & 2.1 & 1.0 & -.3 & .9 \\
\hline 401 & & 1 & 1 & 40.9 & 11.7 & -2.8 & 1.9 & 2.2 & 1.0 & 6.5 & 1.3 \\
\hline 401 & & 1 & 2 & 21.1 & 10.0 &. .9 & 1.9 & 2.3 & 1.1 & 4.4 & 1.1 \\
\hline 401 & & 2 & 1 & 17.7 & 13.0 & -2.3 & 2.2 & 3.6 & 1.5 & 8.9 & 1.5 \\
\hline 401 & & 6 & 1 & -12.2 & 8.0 & .0 & 2.1 & 3.1 & 1.0 & .3 & $.4 *$ \\
\hline 401 & & 6 & 2 & -15.9 & 13.0 & & & 3.1 & 1.7 & 1.4 & 1.0 \\
\hline 401 & & 7 & 1 & 1.7 & 10.5 &.- .3 & 1.5 & -.1 & $1.2^{\circ}$ & 1.9 & 1.1 \\
\hline 401 & & 7 & 2 & -1.1 & 7.1 & .2 & 1.3 & 2.5 & .9 & 1.4 & .8 \\
\hline 401 & & 7 & 3 & 10.6 & 10.0 & -1.6 & 1.6 & -.3 & 1.1 & 2.5 & 1.1 \\
\hline 401 & & 8 & 1 & 33.0 & 2.4 & 4.6 & .4 & 2.4 & .2 & .1 & $.2 *$ \\
\hline 401 & & 8 & 2 & .0 & 10.6 & 1.0 & 1.5 & 1.9 & 1.3 & 2.3 & 1.2 \\
\hline 401 & & 8 & 3 & -2.4 & 8.6 & -.3 & 1.5 & .2 & .7 & 1.5 & .9 \\
\hline 403 & & 1 & 1 & 17.3 & 0.4 & -1.6 & 2.0 & 2.3 & .9 & 4.9 & 1.1 \\
\hline 403 & & 2 & 1 & 2.5 & 0.6 & .2 & 1.8 & .4 & .8 & 3.9 & 1.0 \\
\hline 105 & & 1 & 1 & 57.0 & 13.0 & & & 1.9 & 1.7 & 1.0 & $.9 *$ \\
\hline 105 & & 2 & 1 & .0 & 7.8 & & & 1.6 & .8 & .2 & $1.1 \neq$ \\
\hline 405 & & 4 & 1 & 31.0 & 9.2 & & & 1.2 & 1.0 & .4 & $9.2 *$ \\
\hline 405 & & 4 & 2 & 53.0 & 17.0 & & & 3.8 & 1.7 & 1.0 & $1.1 *$ \\
\hline 407 & & 1 & 1 & 21.3 & 13.5 & .0 & 1.8 & 3.3 & 1.4 & 3.4 & 1.3 \\
\hline 407 & & 2 & 1 & 7.1 & 9.4 & & & -.3 & 1.2 & -1.1 & $1.0 *$ \\
\hline 409 & & 1 & 1 & 19.8 & 9.3 & -.4 & 1.7 & 1.5 & 1.0 & 4.4 & 1.0 \\
\hline 409 & & 1 & 2 & 129.3 & 17.8 & 1.5 & 2.6 & 9.6 & 2.1 & 10.0 & 1.7 \\
\hline 409 & & 1 & 3 & 15.9 & 13.6 & .3 & 2.0 & .2 & 1.5 & 5.8 & 1.5 \\
\hline 411 & & 1 & 1 & 17.0 & 7.4 & & & 2.9 & 1.0 & -.8 & $.9 *$ \\
\hline
\end{tabular}




\begin{tabular}{|c|c|c|c|c|c|c|c|c|c|c|c|}
\hline STAKE & DISTANCE & SAK & & THO & & & & & & URA & \\
\hline no. & METERS & TYPE & REP. & LEVEL & ERROR & LEVEL & ERROR & LEVEL & ERROR & LEVEL & ERROR \\
\hline 411 & & 1 & 2 & 79.7 & 18.7 & .1 & 2.5 & 3.8 & 1.7 & 10.8 & 1.8 \\
\hline 411 & & 2 & 1 & -6.1 & 7.7 & .5 & 1.7 & .3 & .9 & 1.8 & 1.0 \\
\hline 411 & & 2 & 2 & 39.0 & 15.4 & -1.0 & 2.0 & 3.3 & 1.4 & 5.3 & 1.5 \\
\hline 411 & & 6 & 1 & -1.1 & 9.2 & & & 1.9 & 1.0 & -.3 & $.9 *$ \\
\hline 411 & & 7 & 1 & 5.0 & B.6 & & & 1.8 & 1.1 & .0 & $1.0 *$ \\
\hline 411 & & 7 & 2 & 10.6 & 9.7 & -1.3 & 2.0 & 2.1 & 1.2 & 1.7 & 1.2 \\
\hline 411 & & 7 & 3 & -2.4 & 6.6 & -.5 & 1.9 & 1.1 & .7 & 2.9 & .9 \\
\hline 411 & & 8 & 1 & 11.0 & 12.0 & & & 3.8 & 1.3 & .2 & $1.1 *$ \\
\hline 411 & & B & 2 & -14.1 & 10.9 & 1.3 & 1.8 & 2.9 & 1.2 & 1.9 & 1.2 \\
\hline 111 & & $\theta$ & 3 & .0 & 7.7 & -.1 & 1.6 & .1 & .8 & 3.3 & 1.0 \\
\hline 413 & & 1 & 1 & 16.1 & 9.2 & 1.2 & 1.7 & 2.2 & 1.1 & 2.3 & $1.1 *$ \\
\hline 413 & & 1 & 2 & 44.3 & 16.7 & 2.4 & 2.2 & 2.7 & 1.6 & 5.7 & 1.6 \\
\hline 413 & & 2 & 1 & 24.8 & 12.8 & .3 & 1.9 & 1.9 & 1.6 & 4.8 & 1.0 \\
\hline 415 & & 1 & 1 & 60.0 & 15.0 & & & .0 & 1.7 & .0 & $1.0 *$ \\
\hline 415 & & 1 & 2 & 73.0 & 15.0 & & & 1.0 & 1.9 & 1.0 & $1.1 *$ \\
\hline 415 & & 2 & 1 & 12.0 & 12.0 & & & .8 & 1.5 & 1.0 & $1.2 *$ \\
\hline 417 & & $t$ & 1 & 24.8 & 13.7 & -1.3 & 2.1 & 2.9 & 1.5 & 7.9 & 1.5 \\
\hline 417 & & 1 & 2 & 39.0 & 14.8 & 2.4 & 2.1 & 3.1 & 1.5 & 5.3 & 1.5 \\
\hline 417 & & 2 & 1 & 29.7 & 10.2 & -3.5 & 1.9 & 2.9 & 1.2 & 4.6 & 1.2 \\
\hline 417 & & 4 & 1 & 63.1 & 14.5 & -11.0 & 3.0 & 6.5 & 1.6 & 1.1 & $.5 *$ \\
\hline 419 & & 1 & 1 & 21.0 & 10.4 & -2.7 & 1.9 & 3.3 & 1.1 & 4.8 & 1.2 \\
\hline 419 & & 1 & 2 & 9.9 & 8.8 & 2.5 & 1.6 & 1.8 & 1.0 & .4 & $.9 *$ \\
\hline 419 & & 2 & 1 & 9.9 & 9.1 & -1.3 & 1.7 & 1.1 & .9 & 4.5 & 1.0 \\
\hline 421 & & 1 & 1 & 24.8 & B. 4 & .8 & 1.8 & 2.0 & 1.0 & 4.2 & 1.0 \\
\hline 421 & & 2 & 1 & 37.0 & 8.5 & .6 & 1.6 & 1.5 & 1.0 & 2.0 & 1.0 \\
\hline 421 & & 2 & 2 & 5.3 & 10.8 & 1.0 & 1.5 & -.1 & 1.2 & 1.9 & 1.3 \\
\hline 421 & & 4 & 1 & 7.0 & 10.0 & .0 & 1.6 & 1.7 & 1.3 & 3.8 & 1.0 \\
\hline 421 & & 6 & 1 & 8.7 & 5.4 & .4 & 1.3 & .8 & .8 & 1.2 & .8 \\
\hline 421 & & 6 & 2 & -4.9 & 7.6 & -.4 & 1.6 & 2.8 & 1.0 & 2.0 & 1.0 \\
\hline 421 & & 7 & 1 & 6.2 & 8.1 & 4.0 & 2.0 & .7 & .9 & 3.1 & 1.0 \\
\hline 421 & & 7 & 2 & 8.9 & 10.5 & -3.1 & 1.1 & .6 & 1.5 & 2.5 & 1.2 \\
\hline 421 & & 7 & 3 & -1.7 & 8.9 & & & 1.9 & 1.3 & -.3 & . $8 *$ \\
\hline 421 & & 8 & 1 & 5.0 & 8.9 & 2.6 & 1.6 & 2.5 & .9 & 1.9 & 1.0 \\
\hline 421 & & 8 & 2 & -14.1 & 10.0 & .2 & 1.7 & 1.3 & 1.4 & 2.7 & 1.3 \\
\hline 423 & & 1 & 2 & 38.0 & 12.0 & & & 2.5 & 1.2 & .4 & $1.0 *$ \\
\hline 423 & & 2 & 1 & 1.8 & 10.5 & .5 & 1.6 & .6 & 1.2 & 2.2 & $9.3 *$ \\
\hline 425 & & 1 & 1 & 83.0 & 16.0 & & & 2.3 & 1.8 & -1.3 & $1.0 *$ \\
\hline 425 & & 1 & 2 & 27.0 & 14.0 & 3.7 & 1.8 & -.3 & 1.6 & .2 & $1.0 *$ \\
\hline 425 & & 3 & 1 & 41.0 & 11.0 & 7.1 & 2.0 & 5.8 & 1.3 & 1.3 & $1.1 *$ \\
\hline 427 & & 1 & 1 & 18.6 & 11.7 & -2.6 & 2.3 & 2.2 & 1.2 & 6.2 & 1.2 \\
\hline 427 & & 1 & 2 & 71.2 & 8.3 & .8 & 1.6 & 3.3 & 1.0 & 2.8 & 1.0 \\
\hline
\end{tabular}




\begin{tabular}{|c|c|c|c|c|c|c|c|c|c|c|c|}
\hline STAKE & DISTAHCE & SAY & FLE & THC & & & & & & $\mathbf{U} \mathbf{R}$ & \\
\hline No. & HETERS & TYPE & REP. & LEVEL & ERROR & LEVEL & ERROR & LEVEL & ERROR & LEVEL & ERRBR \\
\hline 427 & & 3 & 1 & 100.3 & 16.1 & -4.7 & 2.6 & 8.8 & 1.5 & .8 & $.5 *$ \\
\hline 429 & & 1 & 1 & 24.8 & 10.9 & .8 & 1.8 & 2.1 & 1.2 & 3.4 & 1.3 \\
\hline 429 & & 2 & 1 & 30.1 & 11.3 & 1.6 & 1.8 & -.3 & 1.5 & 3.9 & 1.2 \\
\hline 429 & & 3 & 1 & 15.0 & 9.0 & 3.0 & 1.5 & 1.2 & 1.0 & .0 & $1.0 *$ \\
\hline 431 & & 1 & 1 & 21.0 & 11.4 & -.1 & 1.3 & 2.8 & 1.0 & 5.5 & 1.2 \\
\hline 431 & & 1 & 2 & 35.4 & 15.8 & -1.3 & 1.8 & 3.6 & 1.4 & 4.6 & 1.4 \\
\hline 431 & & 2 & 1 & 5.3 & 11.0 & & & 4.8 & 1.4 & .8 & $.1 *$ \\
\hline 431 & & 3 & 1 & 129.3 & 22.2 & -12.1 & 3.2 & 10.4 & 2.3 & 1.4 & $.8 *$ \\
\hline 431 & & 6 & 1 & 23.0 & 11.3 & 3.1 & 2.0 & .2 & 1.5 & .1 & $.4 *$ \\
\hline 431 & & 7 & 1 & 12.4 & 9.3 & .2 & 1.8 & .4 & 1.0 & 1.9 & 1.1 \\
\hline 431 & & 7 & 2 & -17.9 & 10.0 & & & .8 & 1.4 & -.1 & $.9 *$ \\
\hline 431 & & 7 & 3 & 1.8 & 11.0 & 2.0 & 1.6 & 2.3 & 1.4 & 1.3 & 1.2 \\
\hline 431 & & 8 & 1 & 14.0 & 7.3 & & & -.0 & .9 & .2 & $.8 *$ \\
\hline 431 & & 8 & 2 & 7.1 & 11.8 & -2.6 & 1.6 & 1.3 & 1.0 & 2.7 & $1.1 \%$ \\
\hline 431 & & 8 & 3 & -13.9 & 11.0 & 1.2 & 1.4 & .6 & 1.4 & -.5 & $1.0 *$ \\
\hline 433 & & 1 & 1 & 12.0 & 13.0 & & & 1.2 & 1.1 & 1.1 & $1.1 *$ \\
\hline 433 & & 1 & 2 & 7.1 & 11.0 & & & .8 & 1.3 & 1.2 & $.9 *$ \\
\hline 435 & & 1 & 1 & 37.1 & 14.0 & -1.6 & 2.1 & 1.5 & 1.3 & 7.5 & 1.5 \\
\hline 435 & & 2 & 1 & 20.0 & 8.7 & & & .1 & 1.0 & .5 & $.9 *$ \\
\hline 435 & & 3 & 1 & 106.3 & 20.2 & 7.9 & 2.3 & 11.5 & 2.2 & -.0 & $.7 *$ \\
\hline 437 & & 1 & 1 & 11.1 & 8.7 & -1.6 & 2.1 & 2.1 & .9 & 4.6 & $1.1 *$ \\
\hline 437 & & 1 & 2 & 37.2 & 13.8 & 2.3 & 2.4 & 2.1 & 1.6 & 6.0 & 1.6 \\
\hline 437 & & 2 & 1 & 37.0 & 13.0 & & & 3.1 & 1.5 & -.9 & .9 \\
\hline 437 & & 3 & 1 & 77.9 & 18.5 & -10.2 & 3.2 & 7.1 & 1.8 & 1.3 & .6 \\
\hline 439 & & 1 & 1 & 26.0 & 11.0 & & & 2.9 & 1.1 & 1.4 & 1.0 \\
\hline 439 & & 1 & 2 & 6.2 & 7.1 & & & .0 & .9 & -.3 & .6 \\
\hline 439 & & 3 & 1 & 10.6 & 16.4 & -13.6 & 3.0 & .2 & 1.7 & 1.2 & .6 \\
\hline 441 & & 1 & 2 & 14.7 & 9.8 & .1 & 2.0 & 1.8 & .9 & 4.8 & 1.1 \\
\hline 441 & & 2 & 1 & 17.7 & 14.4 & 1.8 & 2.4 & 1.3 & 1.5 & 7.1 & 1.6 \\
\hline 141 & & 3 & 1 & 30.1 & 13.4 & -3.8 & 2.1 & .4 & 1.5 & .4 & $.5 *$ \\
\hline 441 & & 6 & 1 & 15.9 & 13.1 & 7.7 & 1.6 & 1.3 & 1.3 & -.1 & $.5 *$ \\
\hline 441 & & 6 & 2 & -8.5 & 8.6 & -2.2 & 1.9 & .4 & 1.0 & .2 & $.4 *$ \\
\hline 441 & & 7 & 1 & 40.7 & 12.4 & -.7 & 2.0 & 3.6 & 1.3 & 3.7 & 1.4 \\
\hline 441 & & 7 & 2 & 12.4 & 10.8 & 1.0 & 1.6 & .8 & 1.1 & 3.2 & 1.1 \\
\hline $44 !$ & & 7 & 3 & -7.0 & 10.0 & -.4 & 1.5 & 1.1 & 1.0 & 1.5 & 1.1 \\
\hline 441 & & 8 & 1 & .0 & 2.6 & -.2 & 1.3 & 1.8 & .9 & 1.8 & .9 \\
\hline 441 & & 8 & 2 & -10.5 & 9.0 & 1.3 & 1.5 & 2.3 & 1.1 & 1.9 & $t .1$ \\
\hline 441 & & 8 & 3 & i. 2 & 7.3 & .3 & 1.4 & $\overline{2} .5$ &.$\overline{9}$ & 2.0 & .9 \\
\hline 143 & & 1 & 1 & 19.8 & 8.9 & 2.6 & 2.3 & 2.5 & 1.1 & 5.7 & $1.4 \%$ \\
\hline 443 & & 2 & 1 & 55.7 & 14.0 & 3.2 & 2.5 & 5.8 & 1.2 & B. 0 & 1.4 \\
\hline 443 & & 3 & 1 & 18.6 & 10.7 & -19.9 & 3.3 & 4.6 & 1.2 & 1.6 & .5 \\
\hline
\end{tabular}




\begin{tabular}{|c|c|c|c|c|c|c|c|c|c|c|c|}
\hline \multirow{2}{*}{$\begin{array}{c}\text { STAKE } \\
\text { NO. }\end{array}$} & \multirow{2}{*}{$\begin{array}{l}\text { DISTANCE } \\
\text { METERS }\end{array}$} & \multicolumn{2}{|c|}{ SAMPLE } & \multicolumn{2}{|c|}{ THORIUH } & \multicolumn{2}{|c|}{ RADIUA } & \multicolumn{2}{|c|}{ LEAD } & \multicolumn{2}{|c|}{ URANIUH } \\
\hline & & TYPE & REP. & LEVEL & ERROR & LEVEL & ERROK & LEVEL & ERROR & LEVEL & ERROR \\
\hline 445 & & 1 & 1 & 10.0 & 12.0 & & & 4.4 & 1.4 & 2.2 & $1.1 *$ \\
\hline 445 & & 2 & 1 & -5.2 & 10.0 & & & 2.5 & 1.6 & 1.2 & $1.0 *$ \\
\hline 445 & & 3 & 1 & 34.0 & 11.0 & & & 1.2 & 1.8 & -.3 & $1.0 *$ \\
\hline 447 & & 1 & 1 & 23.5 & 10.0 & .7 & 2.0 & 4.3 & -1.0 & 5.6 & 1.1 \\
\hline 447 & & 2 & 1 & 37.0 & 14.0 & & & 2.7 & 1.5 & .0 & $1.1 *$ \\
\hline 417 & & 3 & 1 & 51.4 & 14.3 & -4.8 & 2.3 & 5.0 & 1.6 & .7 & $.6 *$ \\
\hline 447 & & 9 & 1 & 69.3 & 13.9 & -5.5 & 2.6 & 9.2 & 1.4 & .8 & .5 \\
\hline 149 & & 1 & 2 & 3.5 & 11.0 & & & 3.0 & 1.3 & $-\uparrow .1$ & $1.0 *$ \\
\hline 449 & & 3 & $i$ & 38.0 & 11.0 & 1.0 & -.1 & 3.4 & 1.3 & 2.7 & $.9 *$ \\
\hline 151 & & 1 & 1 & 33.7 & 13.4 & 1.0 & 2.0 & 2.5 & 1.5 & 7.0 & $1.4 *$ \\
\hline 451 & & 1 & 2 & 11.0 & 9.0 & 3.0 & 1.5 & 1.9 & 1.1 & .7 & $.8 *$ \\
\hline 451 & & 6 & 1 & 11.0 & 13.0 & & & .4 & 1.6 & 1.0 & $.9: k$ \\
\hline $45 t$ & & 6 & 2 & -8.5 & 8.1 & & & 1.9 & 1.0 & -2.6 & $1.0 *$ \\
\hline 151 & & 7 & 1 & B.7 & 8.0 & & & 3.4 & 1.0 & .4 & $.8 *$ \\
\hline 451 & & 7 & 2 & 20.0 & 9.3 & & & 3.0 & .9 & -1.5 & .8* \\
\hline 451 & & 7 & 3 & -3.4 & 10.0 & & & 1.5 & 1.2 & -.3 & $.7 *$ \\
\hline 451 & & $\theta$ & 1 & 12.0 & 11.0 & & & .2 & 1.4 & .0 & $1.1 *$ \\
\hline 451 & & $\theta$ & 2 & 8.7 & 7.9 & .3 & 1.6 & .4 & .9 & .9 & $.8 *$ \\
\hline 453 & & 1 & 1 & 13.6 & 8.8 & -.6 & 1.7 & 1.1 & .9 & 2.6 & 1.0 \\
\hline 453 & & 2 & 1 & .0 & 13.3 & -1.6 & 1.8 & -.1 & 1.3 & 4.2 & 1.4 \\
\hline 453 & & 3 & 1 & 47.8 & 17.5 & 1.0 & 2.0 & 2.3 & 1.6 & 7.0 & 1.6 \\
\hline 455 & & 1 & 1 & 11.0 & 9.5 & & & .1 & .9 & .4 & $1.0 *$ \\
\hline 455 & & 1 & 2 & -7.3 & 9.0 & & & 3.6 & 1.1 & .4 & $.8 *$ \\
\hline 455 & & 2 & 1 & 31.0 & 2.6 & 7.2 & .5 & 3.4 & .3 & .3 & $.2 *$ \\
\hline 455 & & 3 & 1 & 14.0 & 9.3 & & & 2.5 & 1.0 & .2 & $1.0 \%$ \\
\hline 457 & & 1 & 1 & 38.9 & 12.0 & 3.3 & 1.5 & .8 & 1.1 & -.0 & .5 \\
\hline 457 & & 2 & 1 & 3.5 & 11.2 & 6.6 & 1.5 & -.3 & 1.2 & -.1 & $1.4 *$ \\
\hline 457 & & 3 & 1 & 105.2 & 16.4 & -3.0 & 2.6 & 11.2 & 1.8 & .7 & $.6 *$ \\
\hline 459 & & 1 & 1 & 17.3 & 10.6 & -2.0 & 1.7 & 4.6 & 1.1 & .2 & $.4 *$ \\
\hline 459 & & 3 & 1 & 19.5 & 16.1 & -5.6 & 2.6 & 4.4 & 1.9 & .7 & $.5 *$ \\
\hline 161 & & 1 & 1 & 3.7 & 10.0 & -2.4 & 2.1 & 1.4 & 1.0 & .4 & $=4 *$ \\
\hline 461 & & 2 & 1 & 17.7 & 14.2 & -12.0 & 2.6 & 1.3 & 1.4 & 1.1 & $.5 *$ \\
\hline 461 & & 7 & 1 & 4.9 & 7.4 & .9 & 1.6 & 3.2 & 1.0 & .2 & $.4 *$ \\
\hline 161 & & 7 & 2 & -1.7 & 11.1 & -3.3 & 2.0 & 1.0 & 1.2 & .5 & $.4 *$ \\
\hline 463 & & 3 & 1 & 24.8 & 12.3 & .5 & 1.7 & 1.3 & 1.3 & 4.3 & 1.3 \\
\hline 165 & & 3 & 1 & 26.0 & 19.3 & -.4 & 2.0 & 4.8 & 1.3 & 4.8 & 1.2 \\
\hline 471 & & 1 & 1 & -7.3 & 7.6 & -.1 & 1.8 & 2.3 & .9 & 4.0 & 1.0 \\
\hline 171 & & 7 & 1 & -1.7 & 11.6 & -.3 & 1.9 & 3.1 & 1.4 & 4.8 & 1.3 \\
\hline 471 & & 7 & 2 & 9.9 & 6.8 & .7 & 1.7 & 1.4 & .9 & 3.0 & .9 \\
\hline 477 & & 1 & 1 & 30.1 & 14.7 & 3.1 & 2.1 & 2.3 & 1.6 & 5.6 & 1.7 \\
\hline 477 & & 1 & 2 & -7.0 & 13.7 & 1.1 & 1.8 & 2.5 & 1.4 & 3.9 & 1.4 \\
\hline
\end{tabular}




\begin{tabular}{|c|c|c|c|c|c|c|c|c|c|c|c|}
\hline STAKE & DISTANCE & SAM & & THO & & & & & & URA & \\
\hline No. & HETERS & TYPE & REP. & LEVEL & ERROR & LEVEL & ERROR & LEVEL & ERROR & LEVEL & ERROR \\
\hline 477 & & 2 & 1 & 17.7 & 13.0 & .6 & 1.9 & 3.1 & 1.3 & 4.2 & 1.4 \\
\hline 477 & & 3 & 1 & 26.0 & 9.8 & -1.0 & 1.8 & 2.1 & .9 & 3.5 & 1.1 \\
\hline 179 & & 1 & 1 & 7.4 & 7.8 & -2.0 & 1.8 & 1.7 & .9 & 4.4 & 1.0 \\
\hline 479 & & 1 & 2 & 52.0 & 11.6 & .8 & 2.2 & 1.2 & 1.1 & 6.9 & 1.2 \\
\hline 479 & & 2 & 1 & 1.8 & 9.9 & -.3 & 1.5 & .2 & 1.2 & 2.7 & 1.0 \\
\hline 479 & & 3 & 1 & 33.7 & 11.3 & 2.7 & 1.5 & 1.5 & 1.2 & 1.5 & 1.2 \\
\hline 481 & & 1 & 2 & 17.3 & 9.4 & .1 & 1.8 & 2.2 & 1.1 & 5.0 & 1.2 \\
\hline 481 & & 1 & 3 & 22.3 & 11.2 & -.6 & 2.1 & 2.9 & 1.1 & 5.3 & 1.2 \\
\hline 481 & & 2 & 1 & 10.6 & 10.6 & 3.3 & 1.8 & 3.8 & 1.3 & 2.3 & 1.2 \\
\hline 481 & & 3 & 1 & 11.1 & 10.1 & .0 & 1.9 & 3.0 & 1.1 & 3.1 & 1.0 \\
\hline 481 & & 6 & 1 & 1.2 & 9.5 & -.7 & 1.8 & 1.0 & 1.0 & 3.4 & 1.1 \\
\hline 481 & & 6 & 2 & -5.2 & 10.5 & -.5 & 1.6 & .8 & 1.4 & 4.2 & 1.4 \\
\hline 481 & & 7 & 1 & 1.8 & 12.9 & .1 & 1.7 & -1.2 & 1.3 & 3.7 & 1.3 \\
\hline 481 & & 7 & 2 & -6.1 & 7.7 & .2 & 1.8 & -.0 & .8 & 2.9 & 1.1 \\
\hline 481 & & 8 & 1 & 2.5 & 8.2 & 1.4 & 1.9 & 2.6 & .9 & 3.0 & 1.0 \\
\hline 483 & & 1 & 1 & 14.9 & 9.3 & 3.7 & 2.1 & 3.4 & 1.0 & 3.6 & 1.0 \\
\hline 483 & & 1 & 2 & 44.3 & 14.1 & -1.5 & 1.9 & 4.8 & 1.6 & 5.2 & 1.3 \\
\hline 483 & & 2 & 1 & 12.4 & 8.9 & -.3 & 1.8 & 3.0 & 1.0 & 4.0 & 1.1 \\
\hline 483 & & 2 & 2 & -4.9 & 8.6 & 1.0 & 1.6 & 1.1 & .9 & 2.2 & 1.0 \\
\hline 483 & & 3 & 1 & 76.2 & 14.5 & -.3 & 2.2 & 1.3 & 1.5 & 7.2 & 1.6 \\
\hline 485 & & 1 & 1 & 17.7 & 12.0 & .2 & 1.6 & 3.3 & 1.4 & 2.7 & 1.3 \\
\hline 485 & & 1 & 2 & 7.1 & 12.8 & .1 & 1.9 & .8 & 1.4 & 4.5 & 1.3 \\
\hline 485 & & 2 & 1 & 21.3 & 13.7 & 2.2 & 2.1 & .6 & 1.4 & 5.2 & 1.4 \\
\hline 485 & & 3 & 1 & 35.4 & 14.4 & 3.7 & 2.3 & .0 & 1.6 & 6.4 & 1.9 \\
\hline 487 & & 2 & 1 & 7.1 & 10.3 & 1.5 & 2.1 & 1.5 & 1.4 & 5.0 & 1.4 \\
\hline 487 & & 2 & 2 & 54.5 & 14.7 & -9.9 & 2.3 & 2.5 & 1.5 & 12.9 & 1.6 \\
\hline 487 & & 3 & 1 & 37.1 & 11.7 & -6.0 & 2.1 & 4.1 & 1.2 & 8.0 & 1.2 \\
\hline 489 & & 1 & 1 & -7.0 & 11.2 & 2.5 & 1.7 & 2.7 & 1.5 & 2.3 & 1.3 \\
\hline 489 & & 1 & 2 & 7.1 & 10.3 & .0 & 1.3 & 1.5 & 1.1 & 2.9 & 1.1 \\
\hline 489 & & 2 & 1 & 28.3 & 14.2 & 1.0 & 1.9 & 1.5 & 1.6 & 1.9 & 1.4 \\
\hline 489 & & 3 & 1 & 64.4 & 14.3 & 5.7 & 2.2 & 6.3 & 1.4 & 5.2 & 1.4 \\
\hline 489 & & 9 & 1 & 123. & 16.9 & 2.0 & 3.1 & 11.9 & 1.7 & 12.4 & 1.7 \\
\hline 491 & & 1 & 1 & -5.2 & 10.5 & 1.0 & 1.7 & 3.6 & 1.3 & 2.3 & 1.3 \\
\hline 491 & & 1 & 2 & 6.2 & 5.9 & 1.2 & 1.5 & -.0 & .8 & .9 & .8 \\
\hline 491 & & 2 & 1 & 8.7 & 0.5 & -4.0 & 2.2 & 3.0 & 1.0 & 7.5 & 1.2 \\
\hline 491 & & 6 & 1 & .0 & 0.8 & 1.4 & 2.0 & 2.8 & 1.0 & 4.1 & 1.1 \\
\hline 491 & & 6 & 2 & -2.4 & 8.8 &.$- B$ & 1.8 & 1.4 & .9 & 2.5 & 1.0 \\
\hline 491 & & 7 & 1 & 3.5 & 10.0 & 2.6 & 1.3 & .8 & 1.1 & .8 & 1.0 \\
\hline 491 & & 7 & 2 & 9.9 & 7.4 & 1.2 & 1.6 & -.9 & .8 & 3.5 & .9 \\
\hline 491 & & 7 & 3 & 10.6 & 10.9 & .7 & 1.6 & .6 & 1.3 & 1.9 & 1.2 \\
\hline 491 & & 8 & 1 & -5.2 & 10.2 & .6 & 1.6 & 1.0 & 1.0 & 2.0 & 1.2 \\
\hline
\end{tabular}




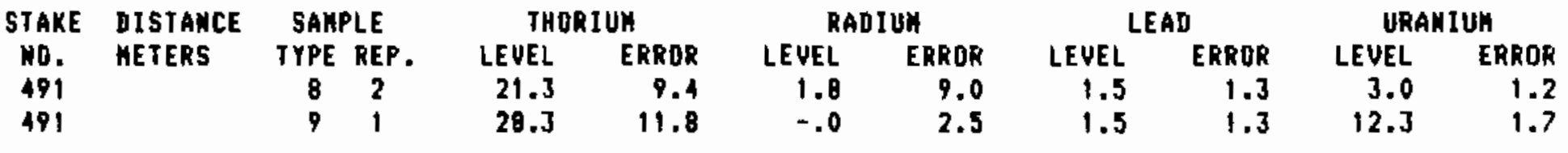




\section{APPENDIX C}

230 Th CONCENTRATIONS PLOTTEO VERSUS RIO PUERCO DISTANCE DOWNSTREAM FROM SPILL SITE 


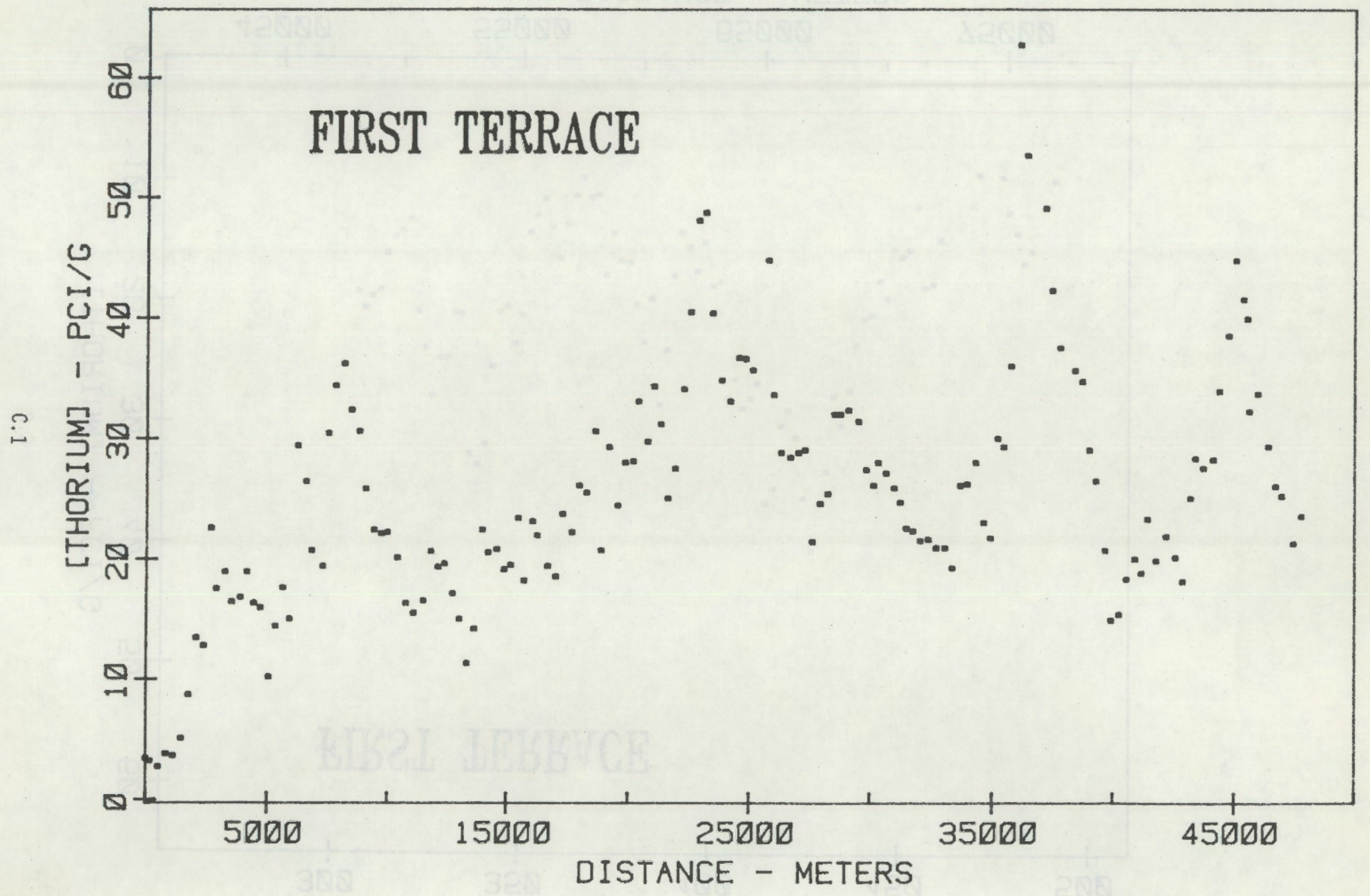




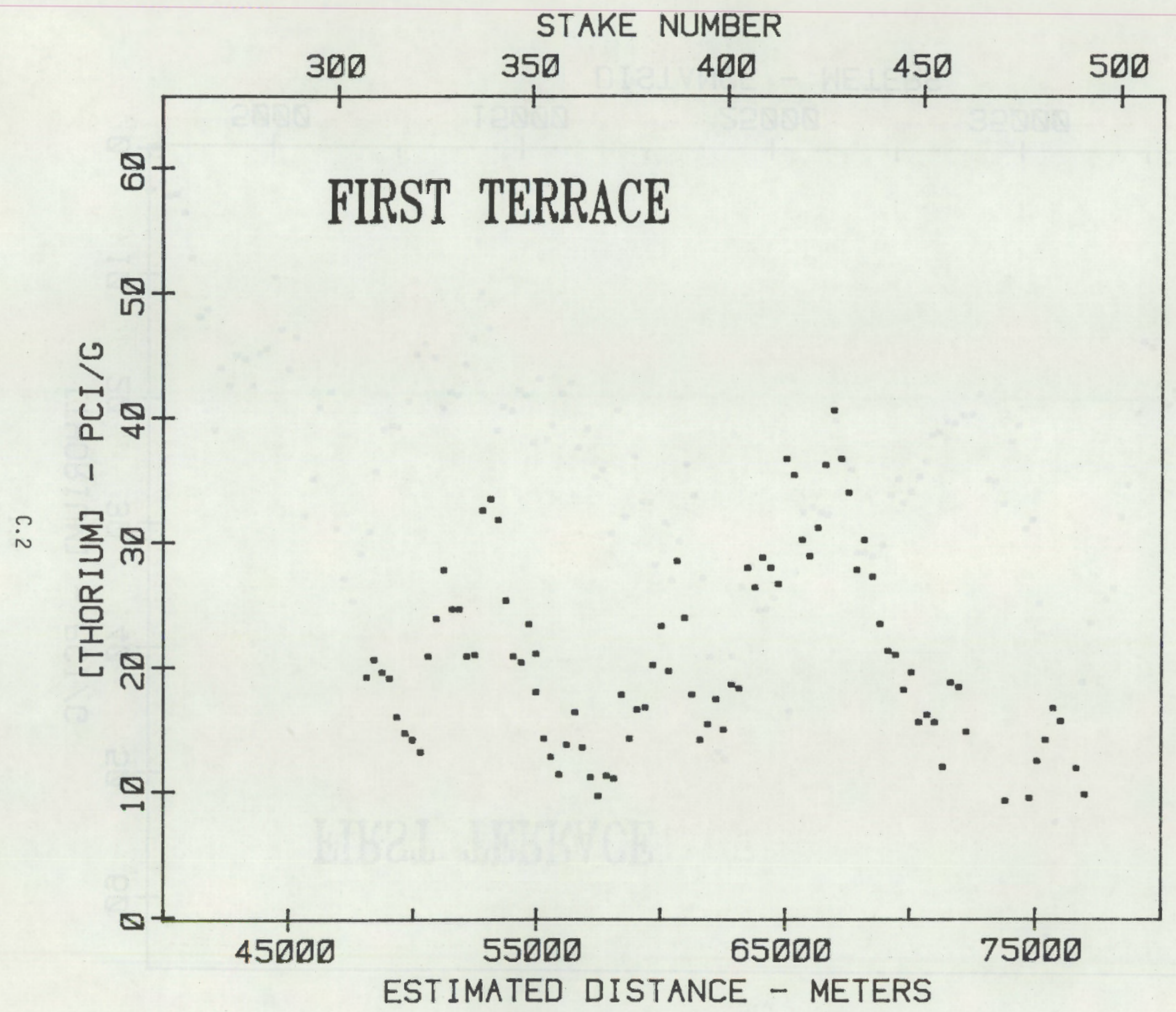




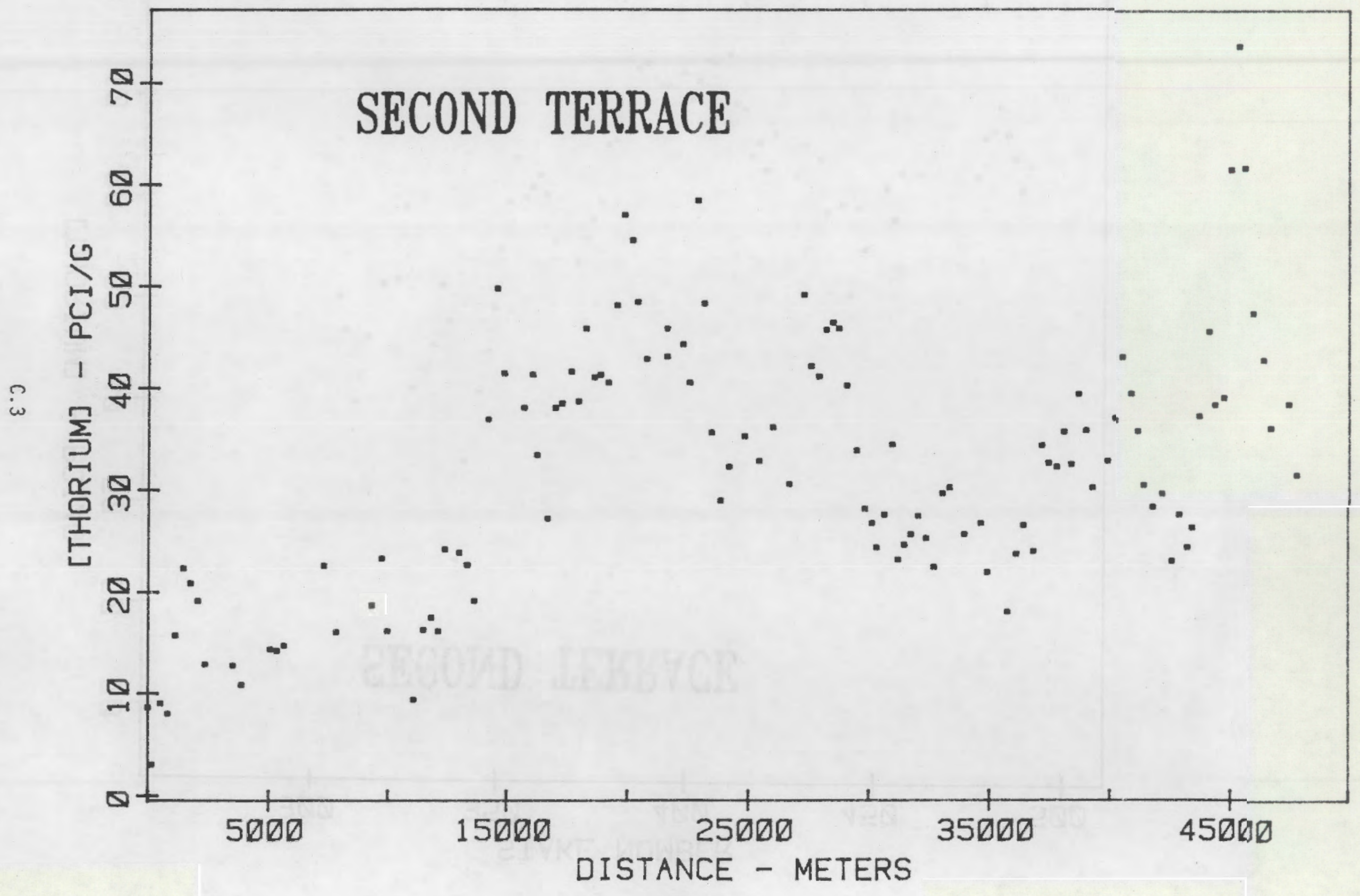




\section{CONCENTRATED AREA}

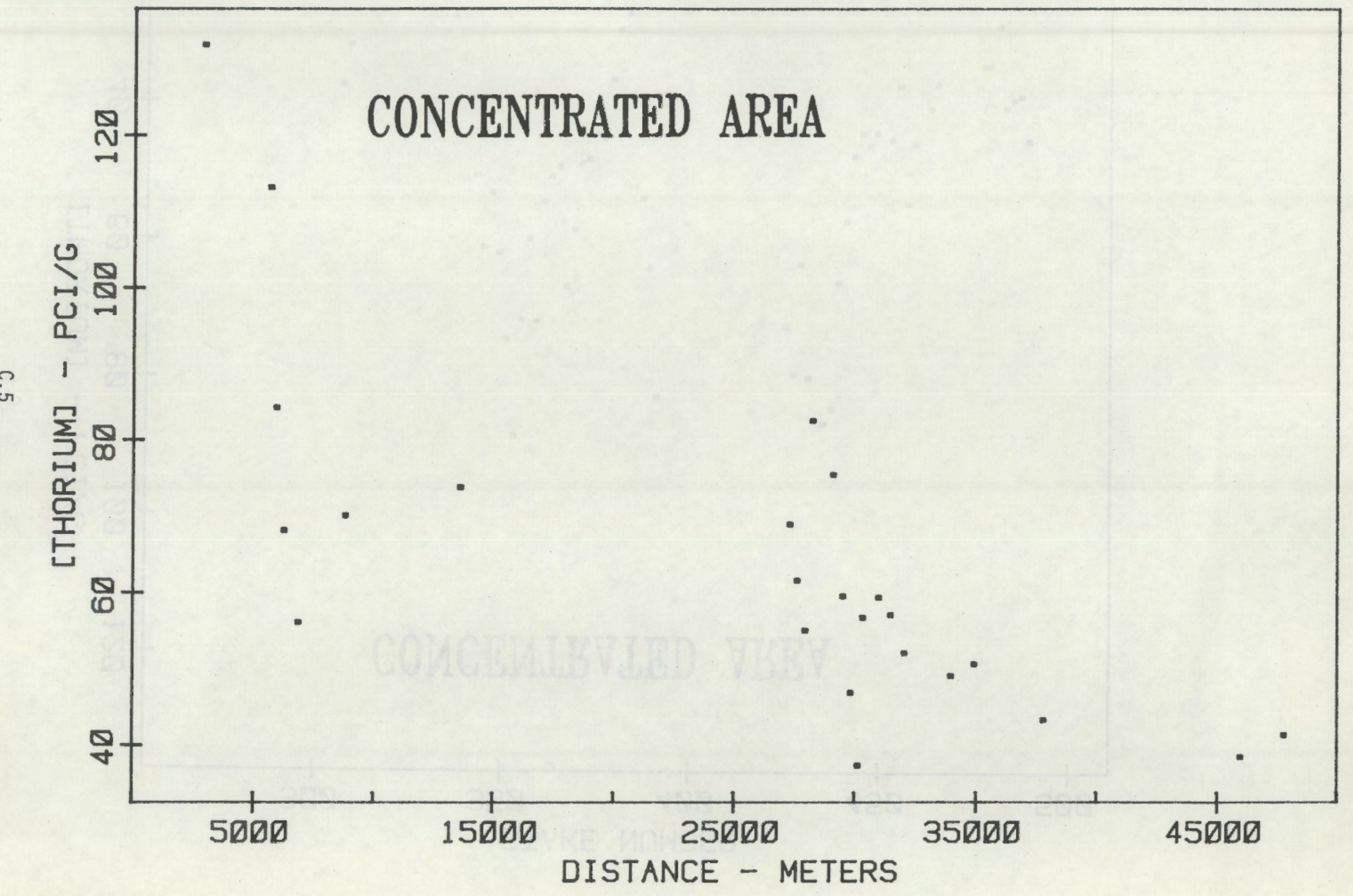




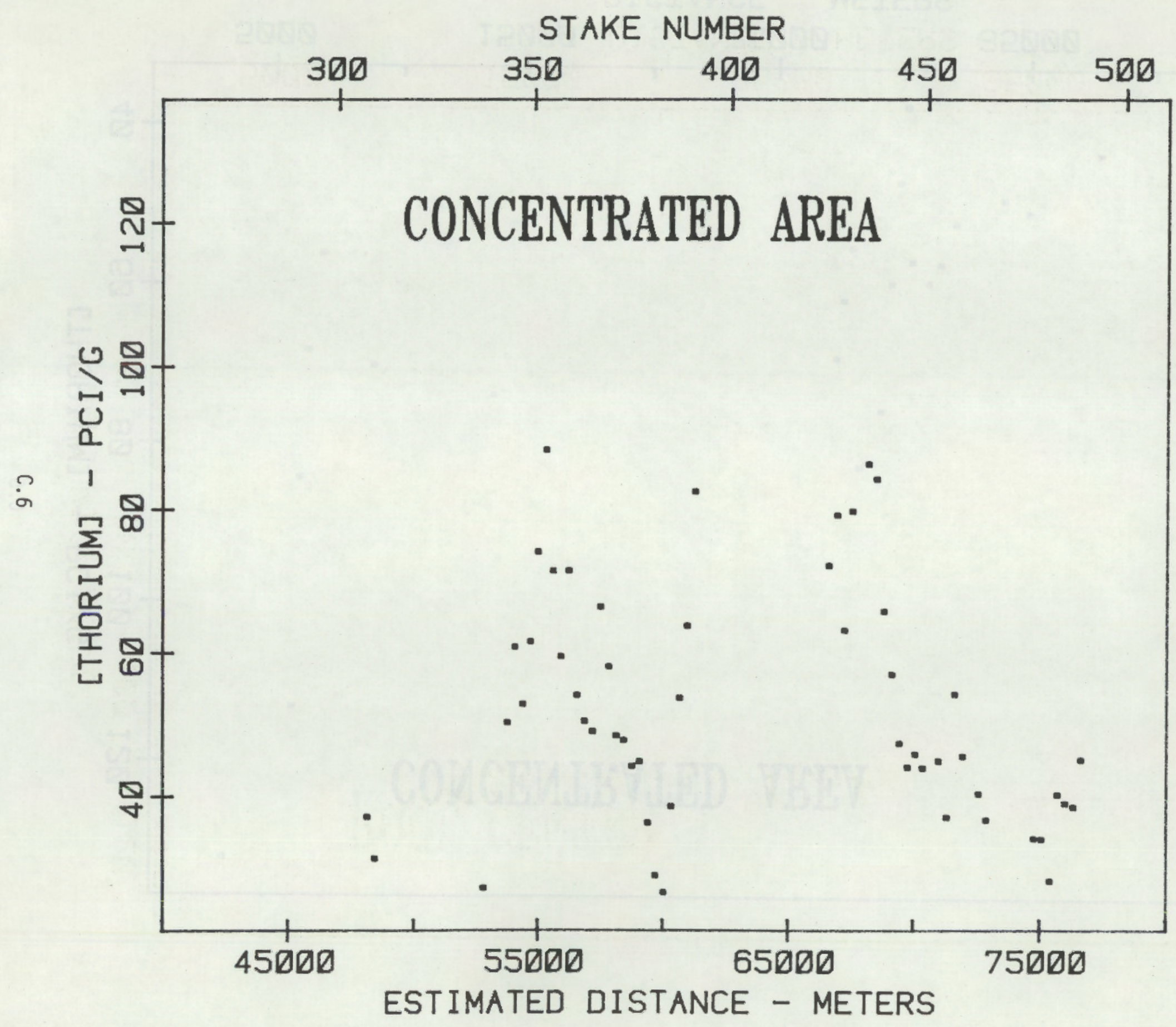




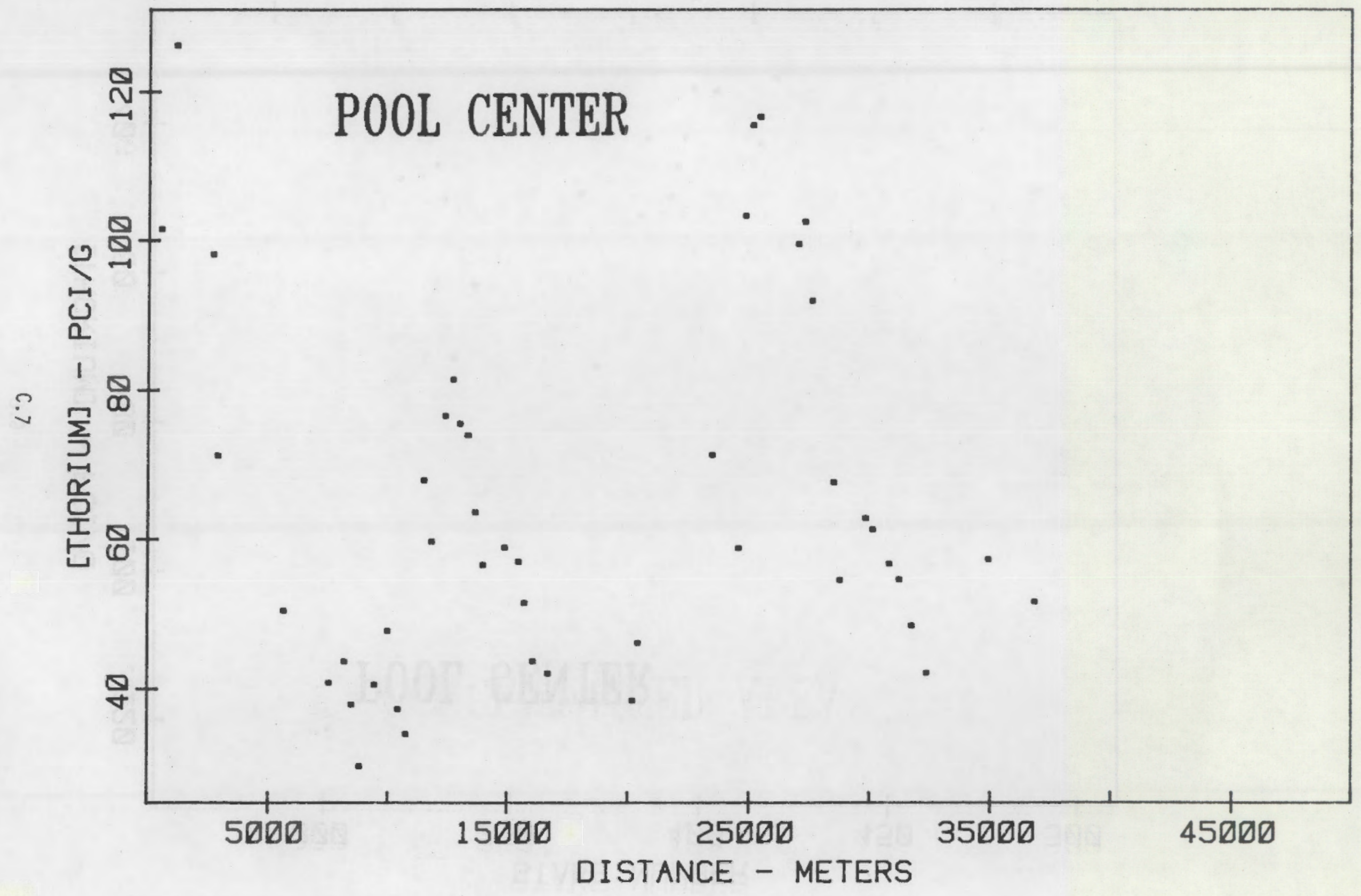




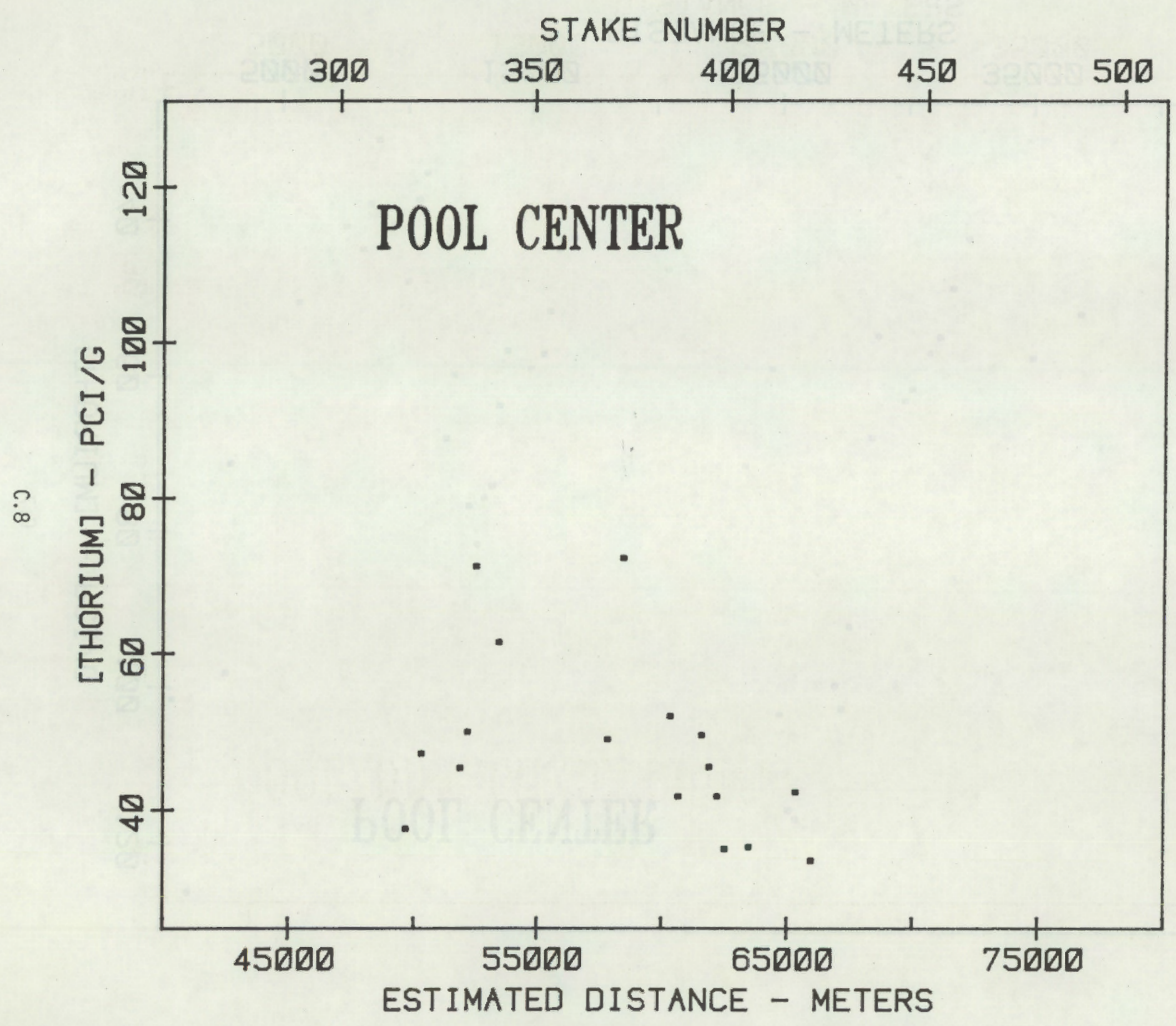




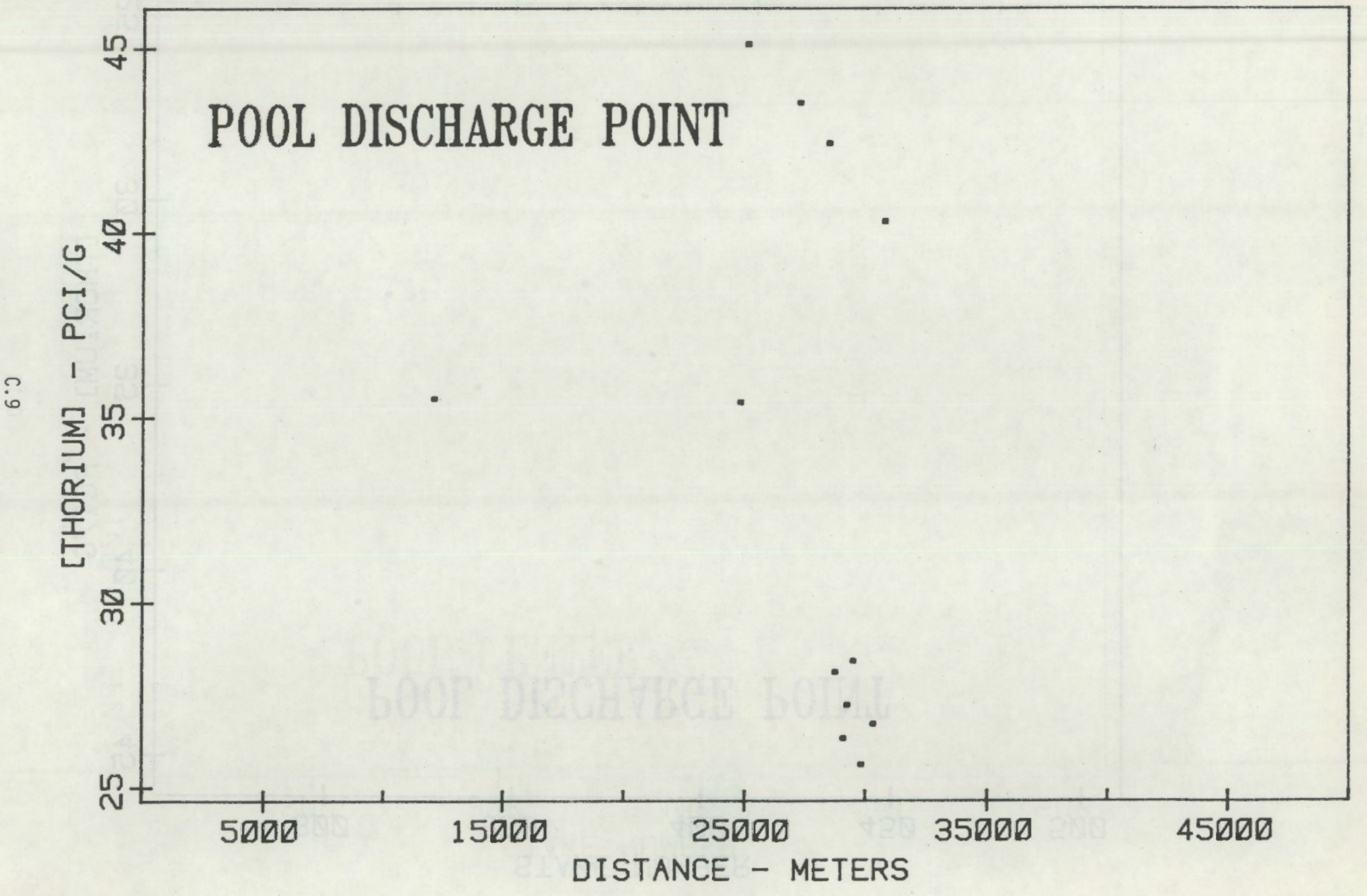




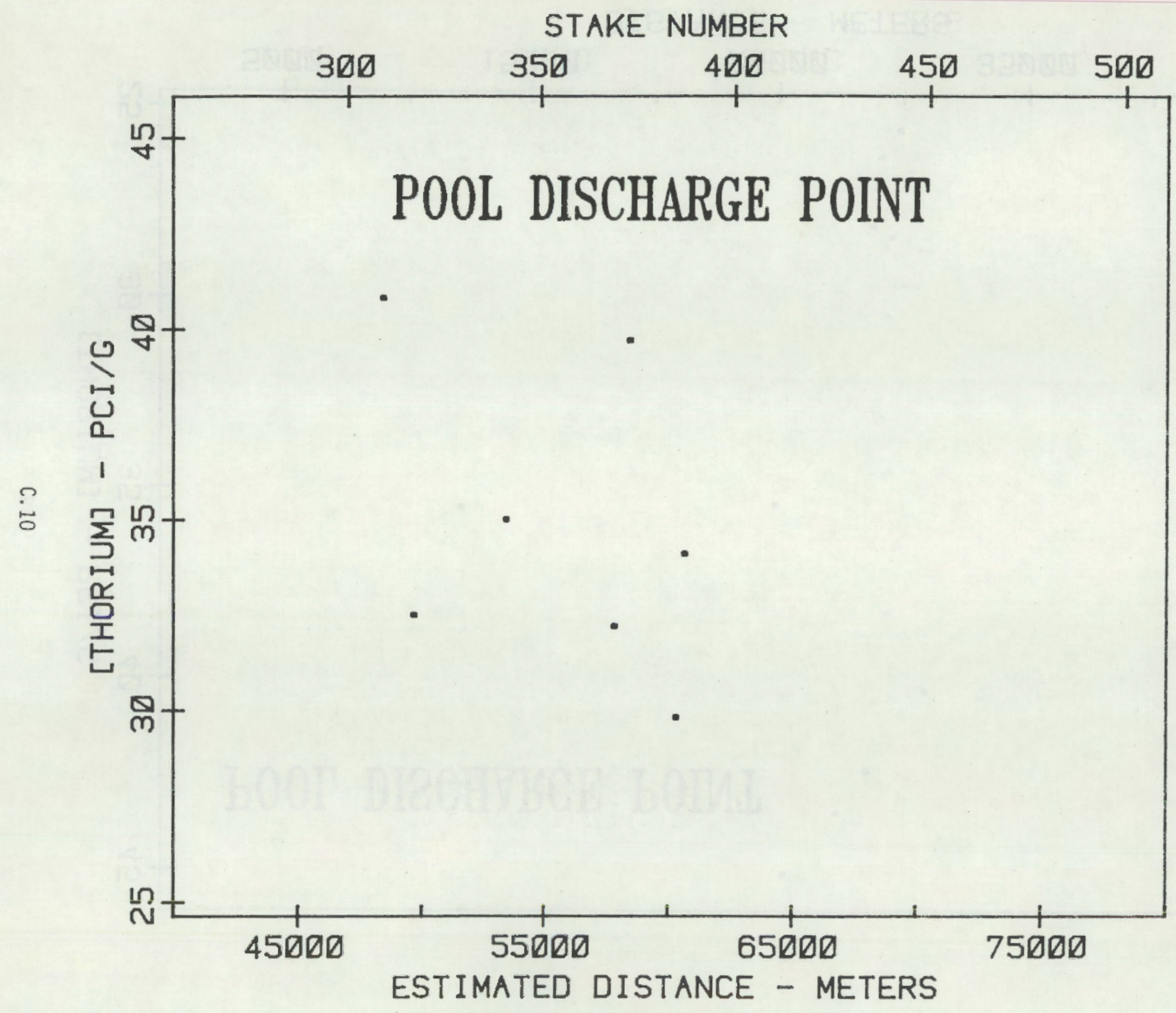




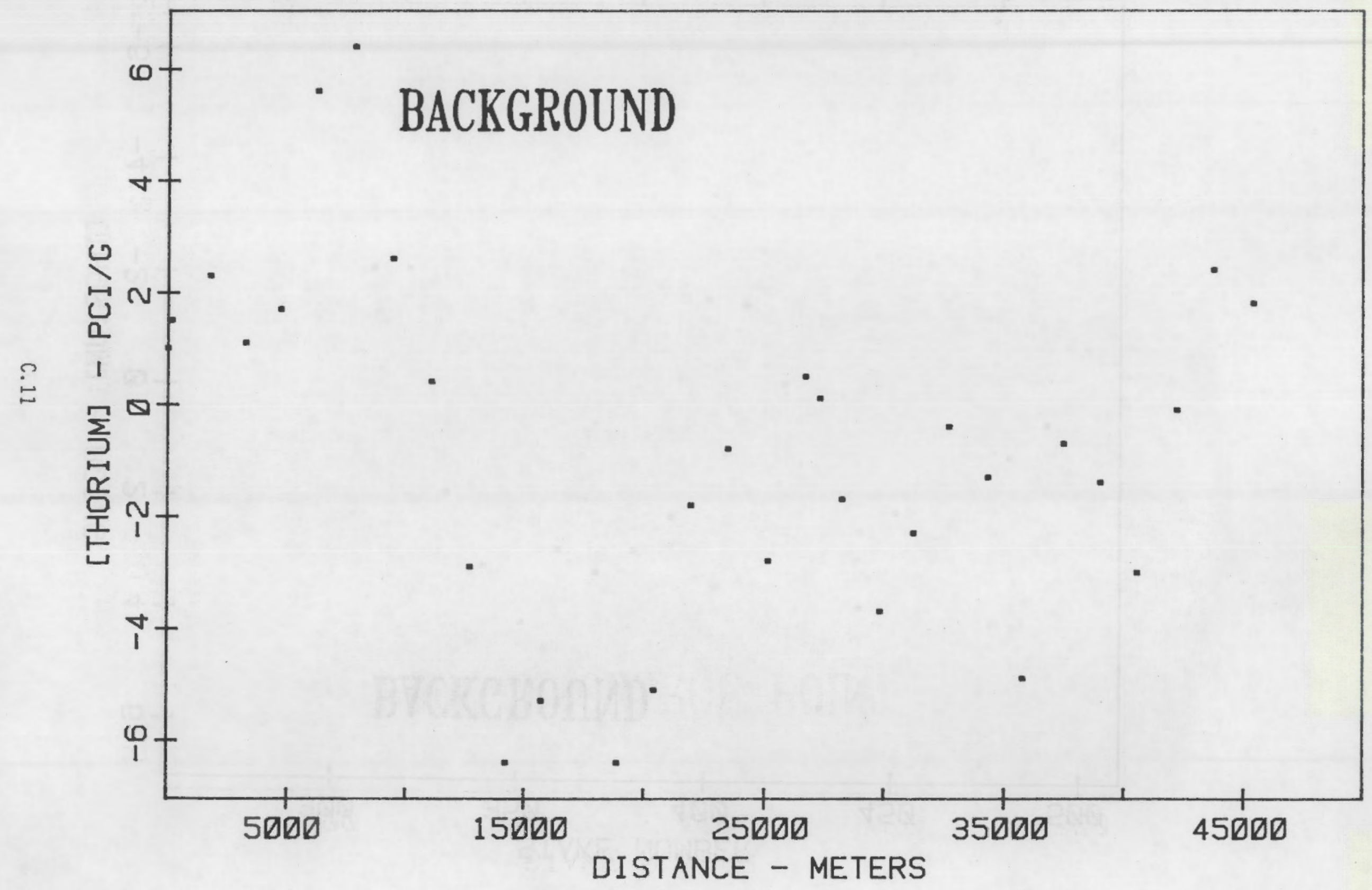




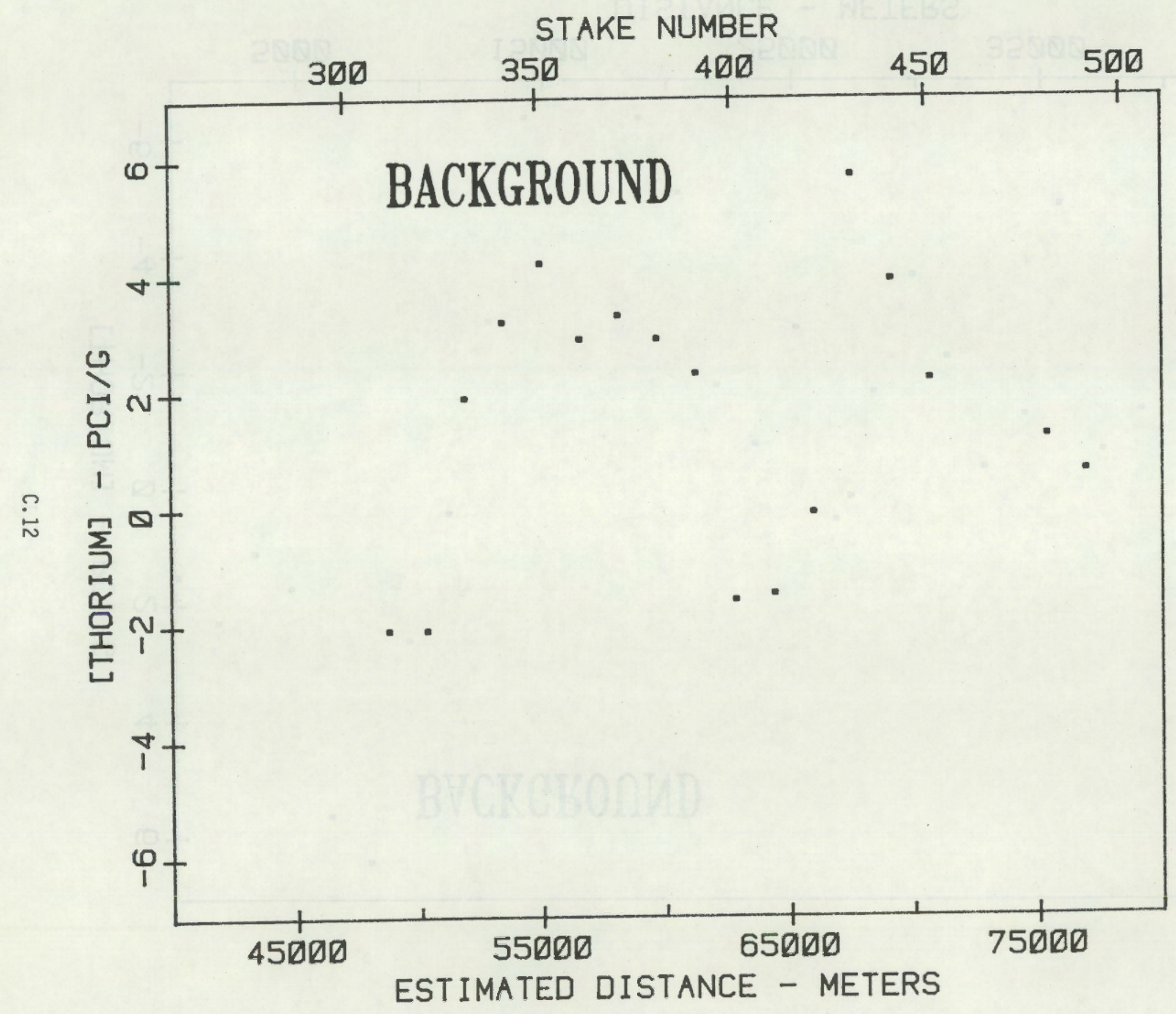




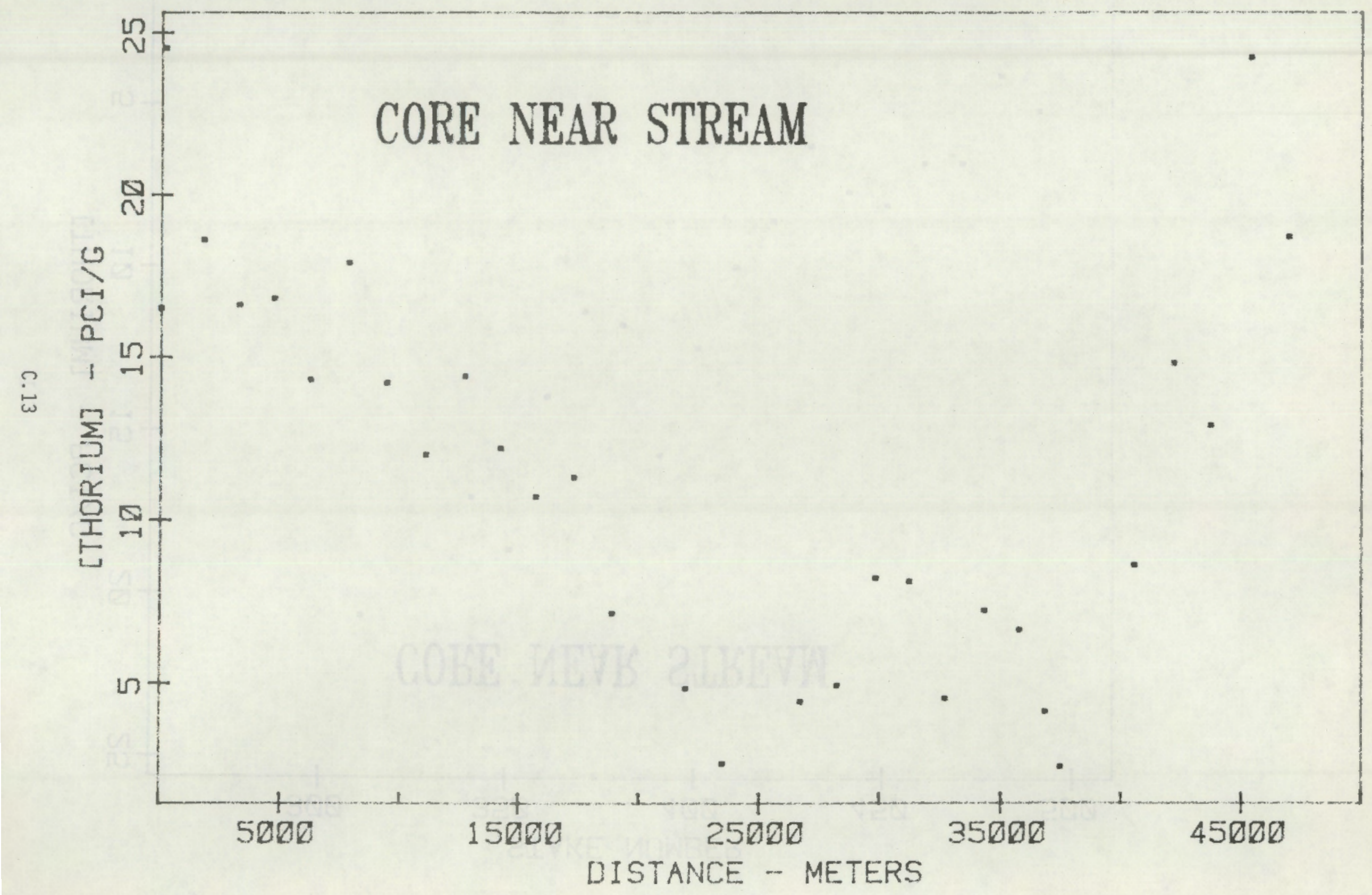




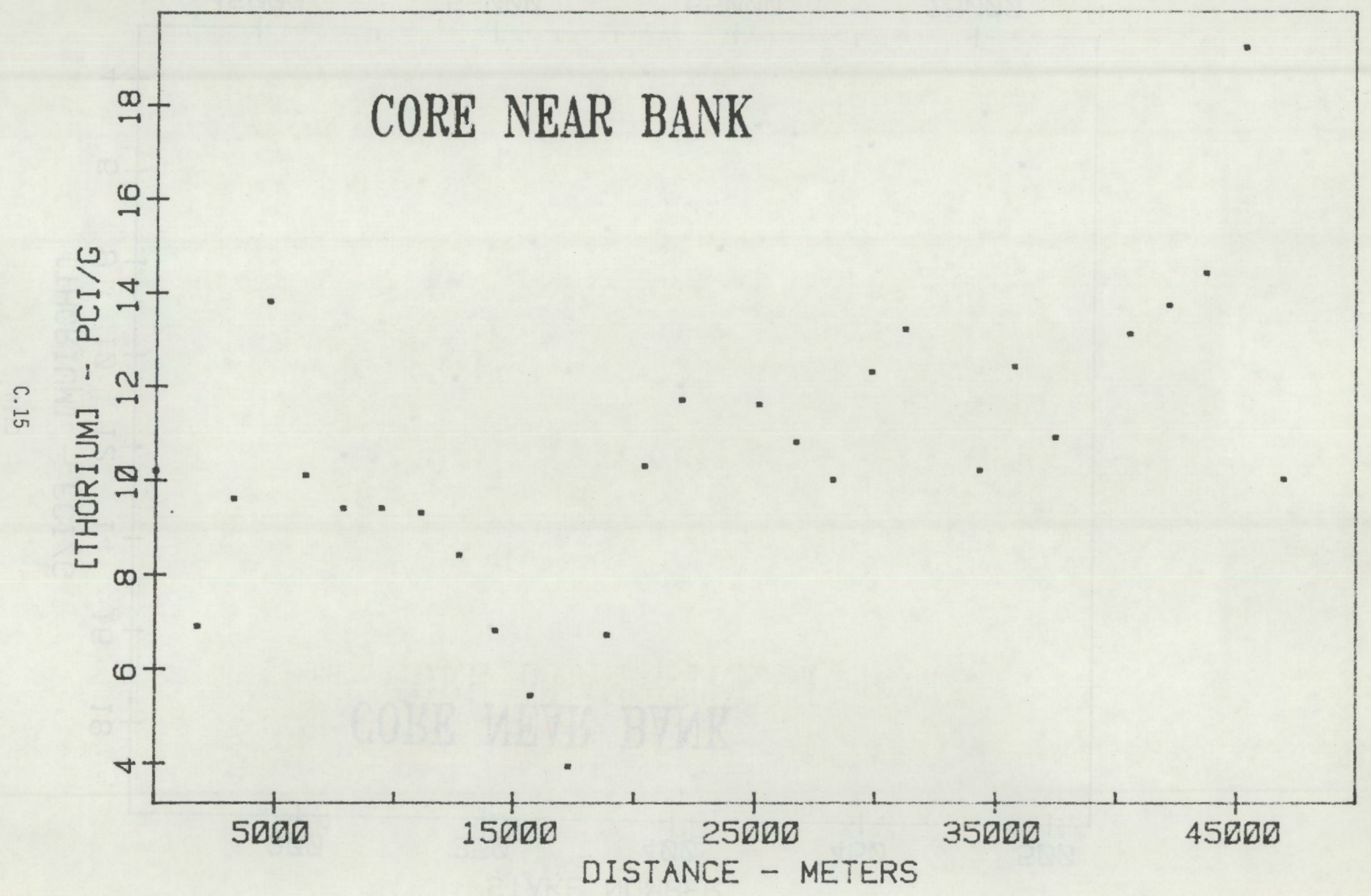




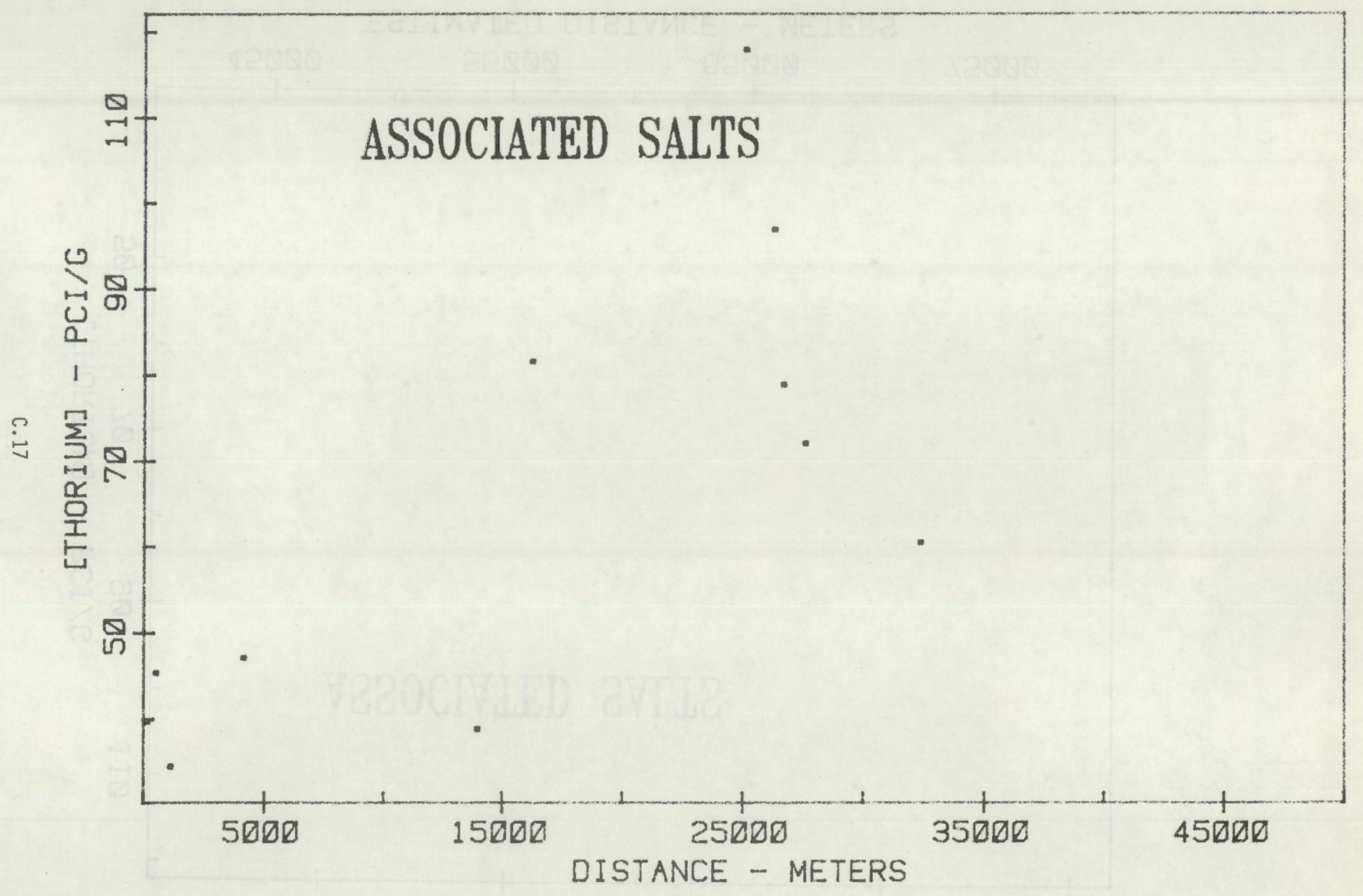


STAKE NUMBER

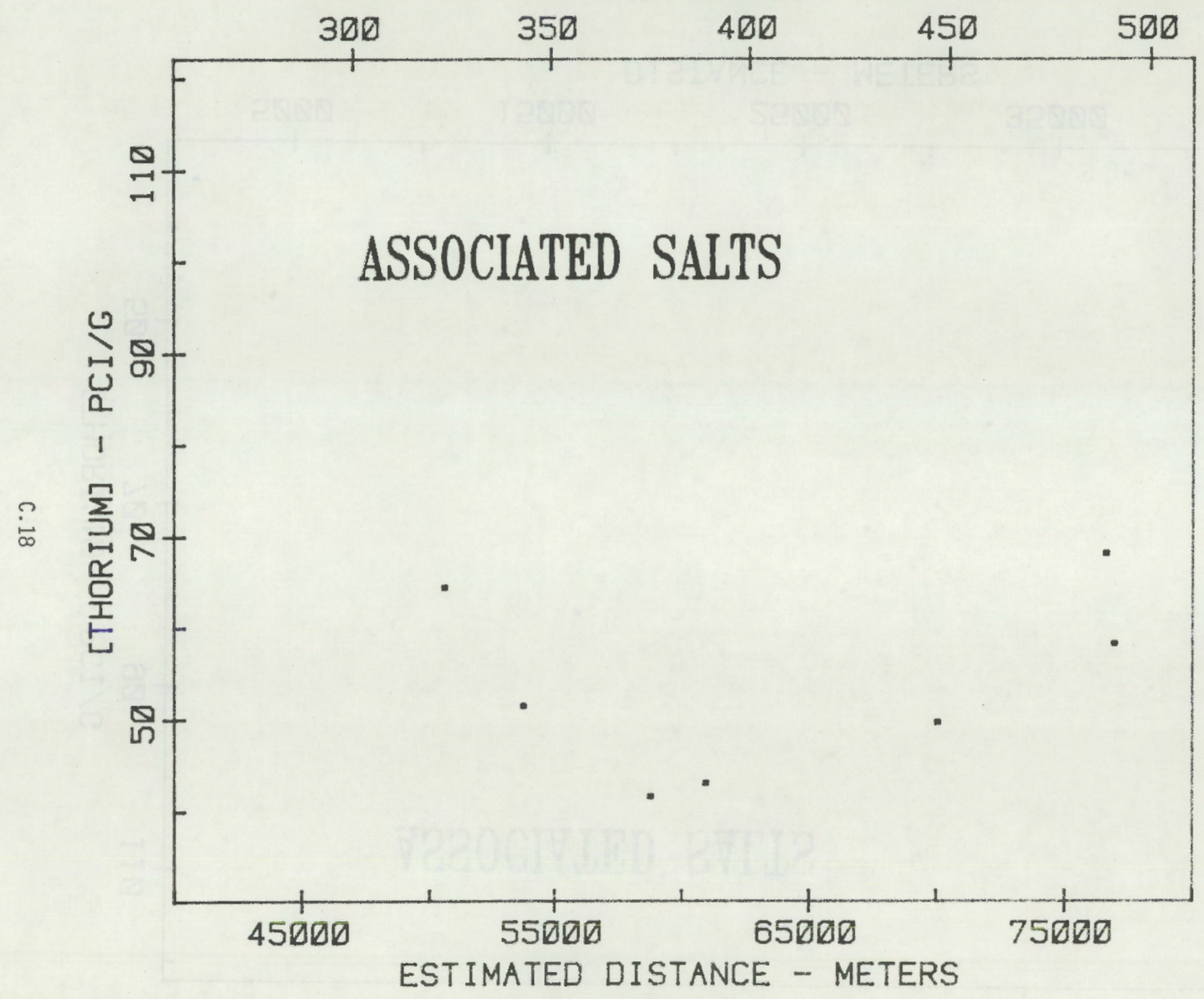




\section{APPENDIX D}

RIO PUERCO SAMPLING LOCATIONS 


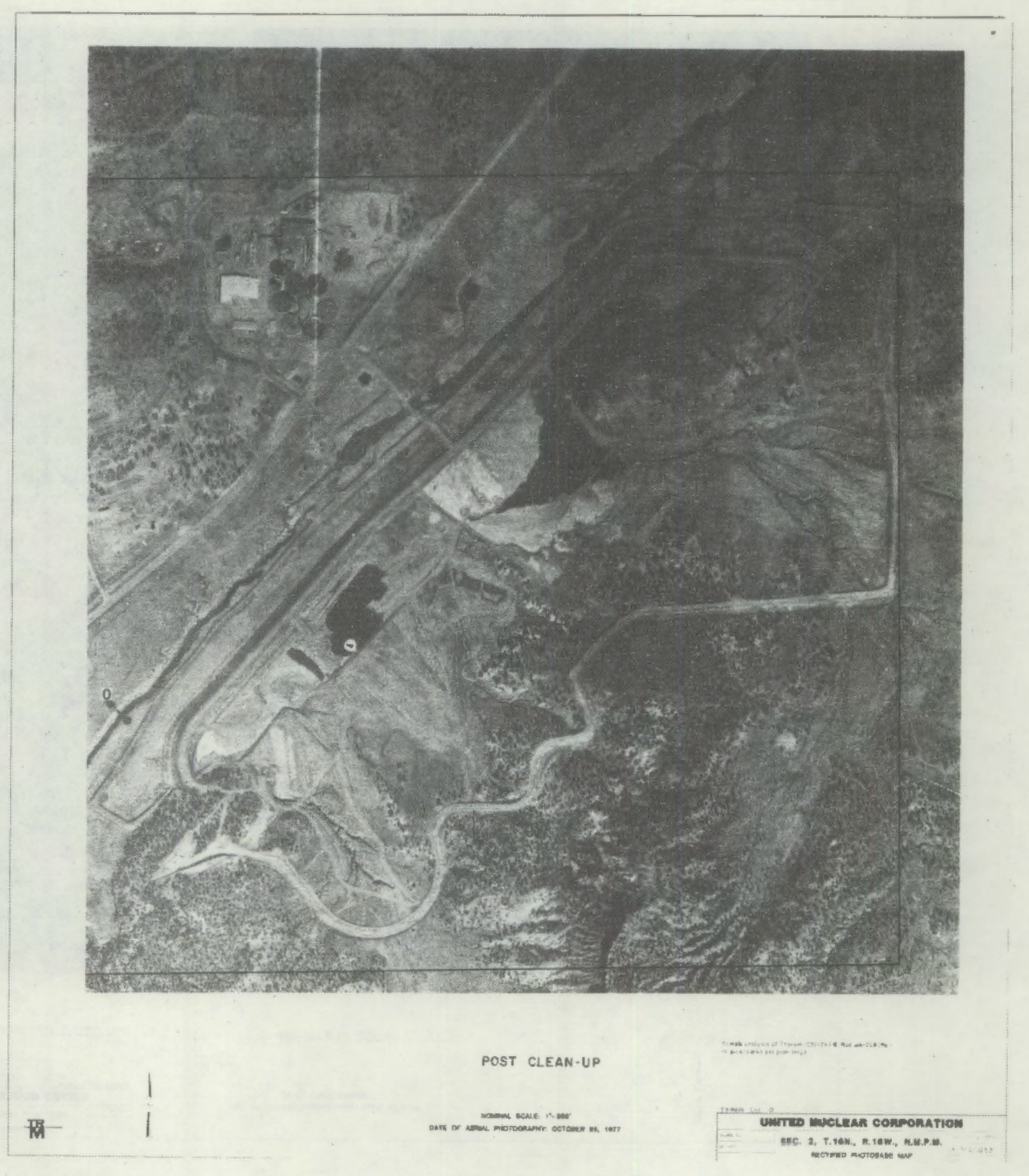

D. 1 


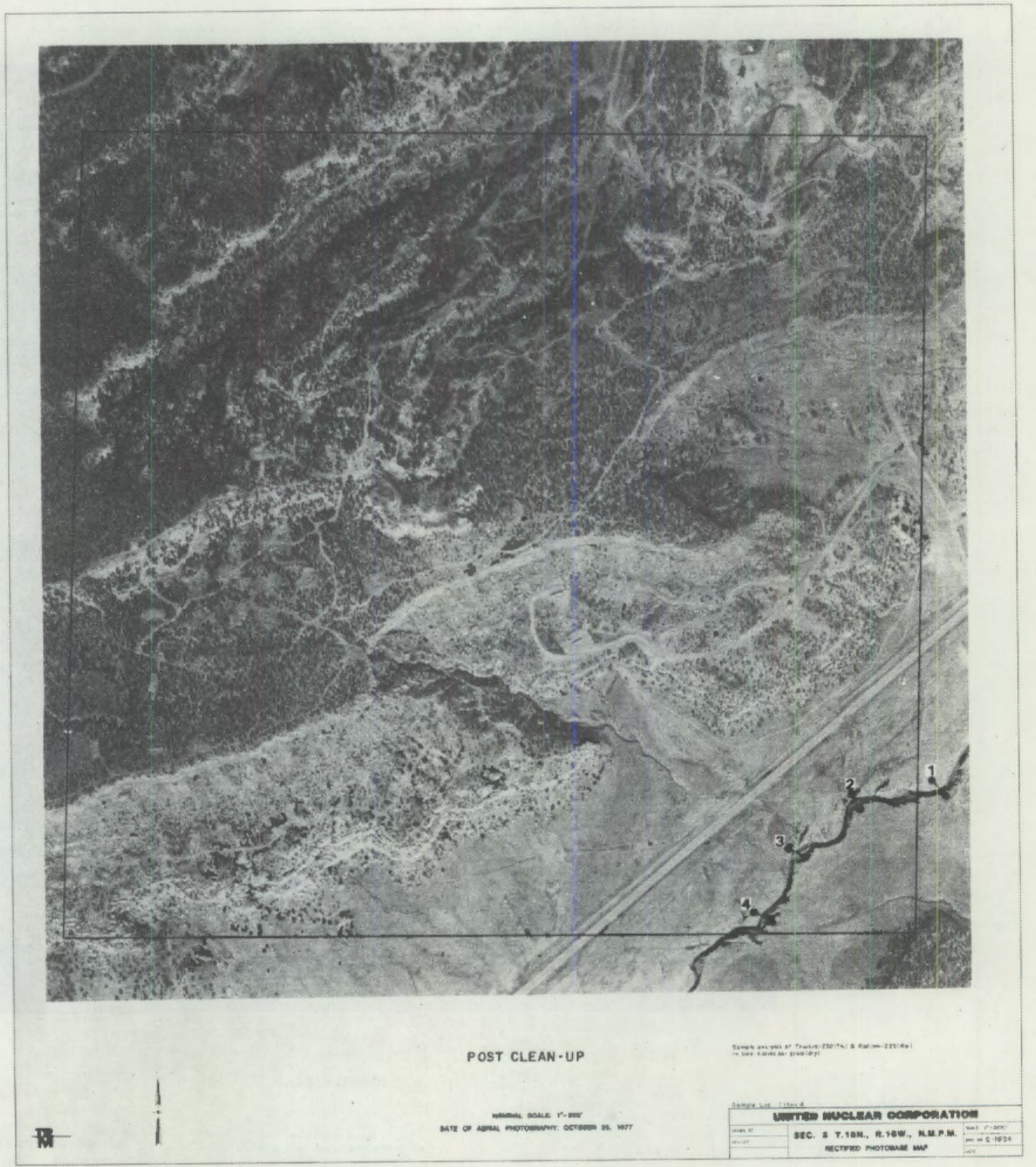




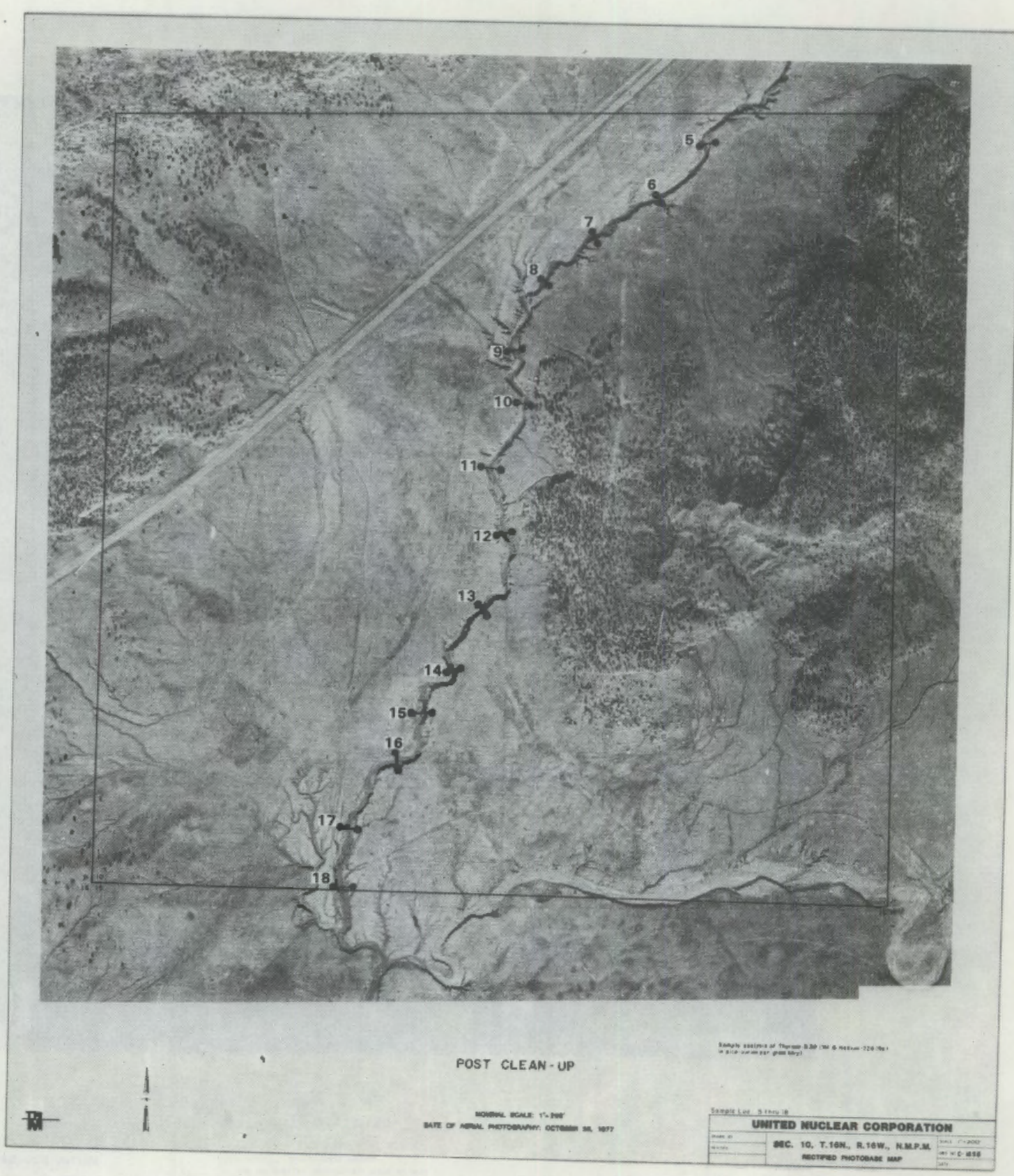

D. 3 


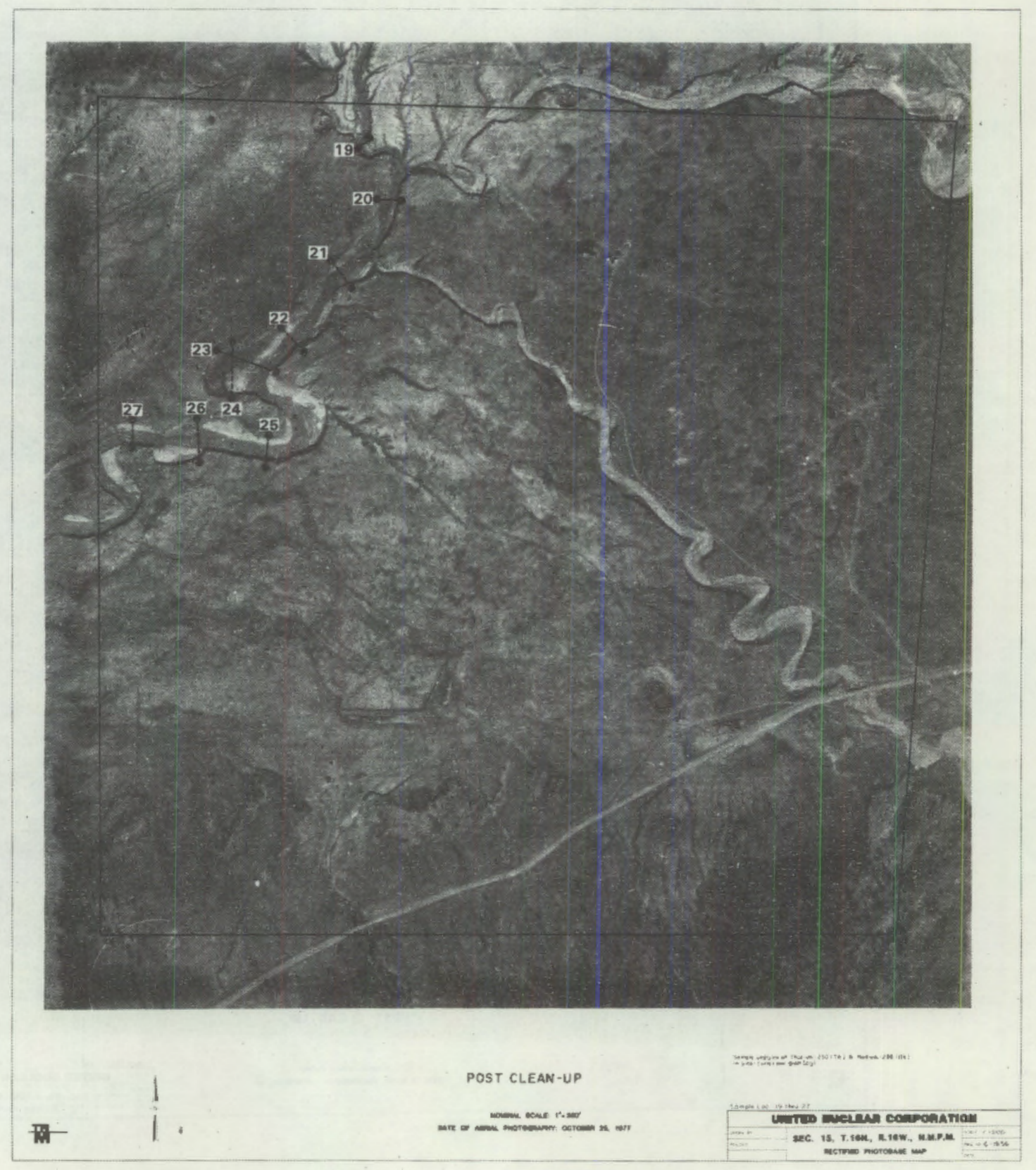

0.4 


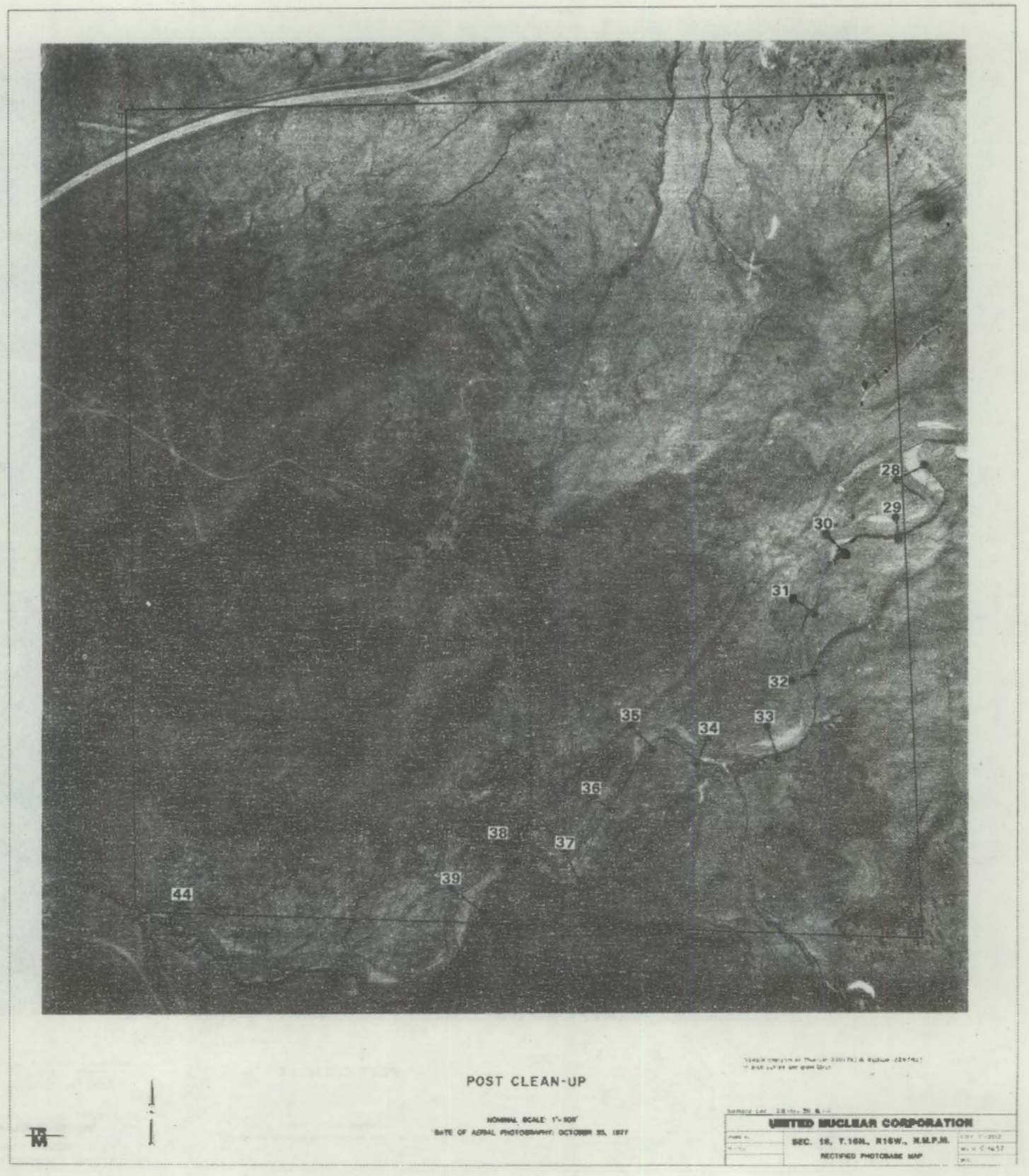

D. 5 


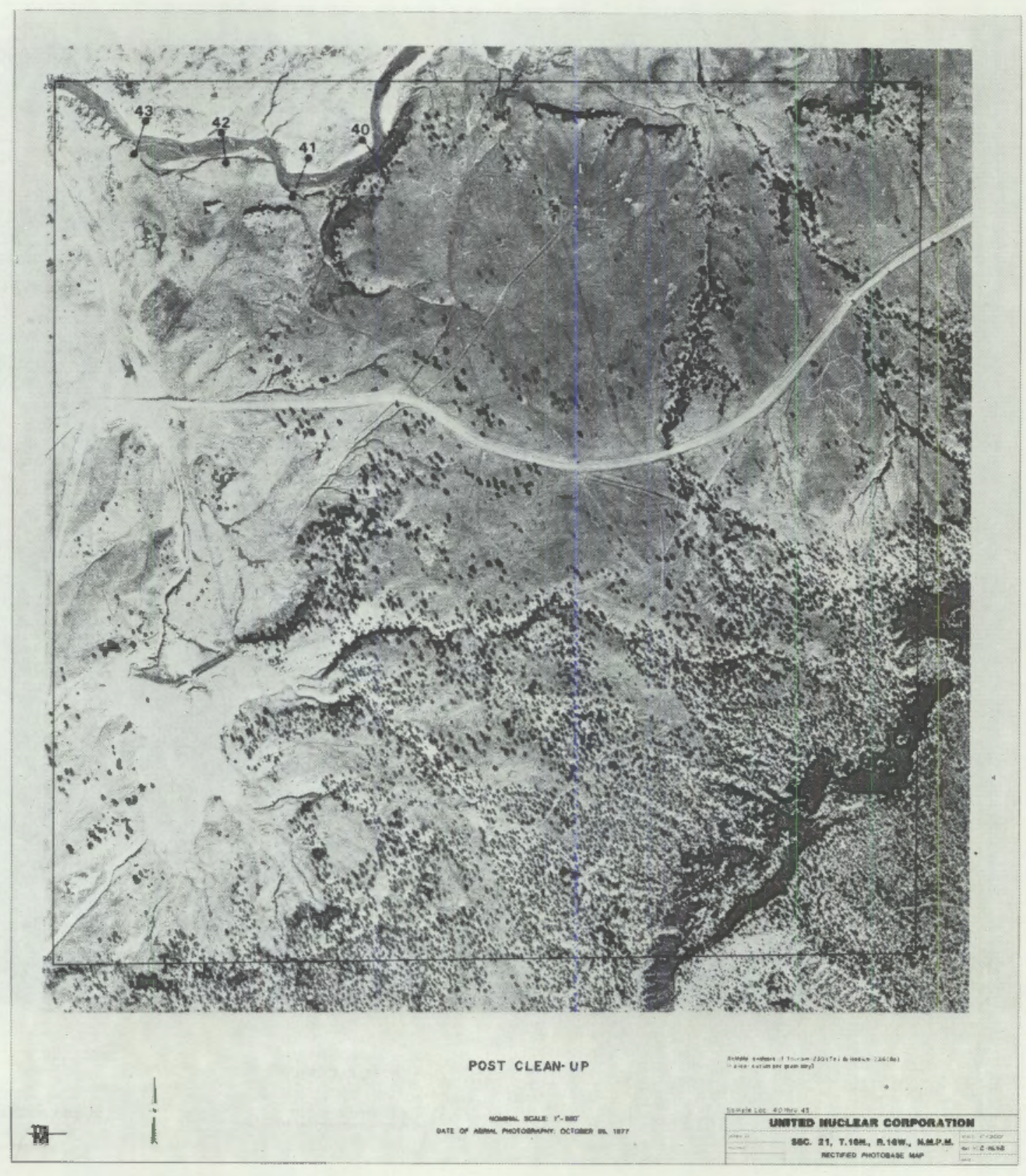

D. 6 


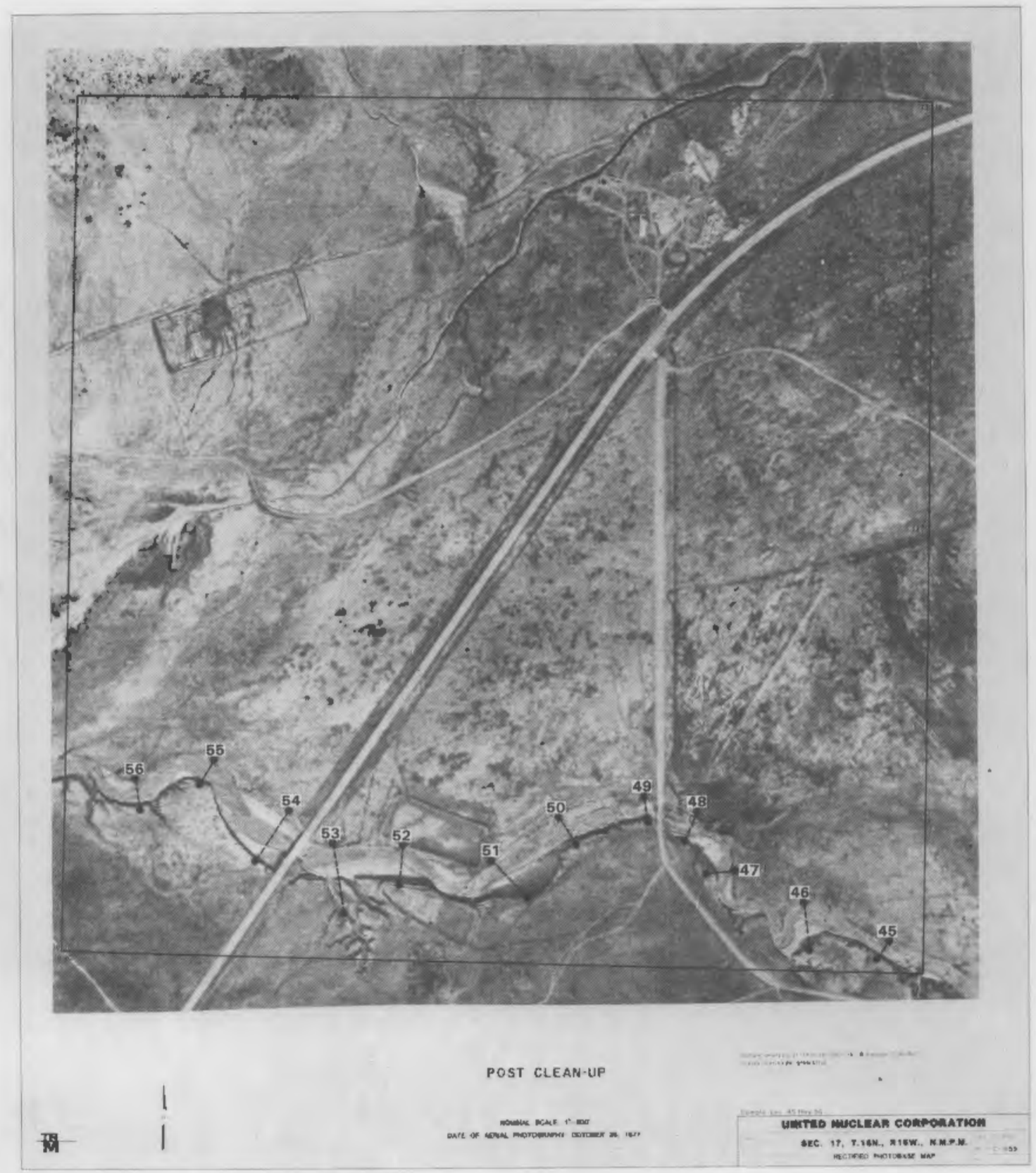

D. 7 


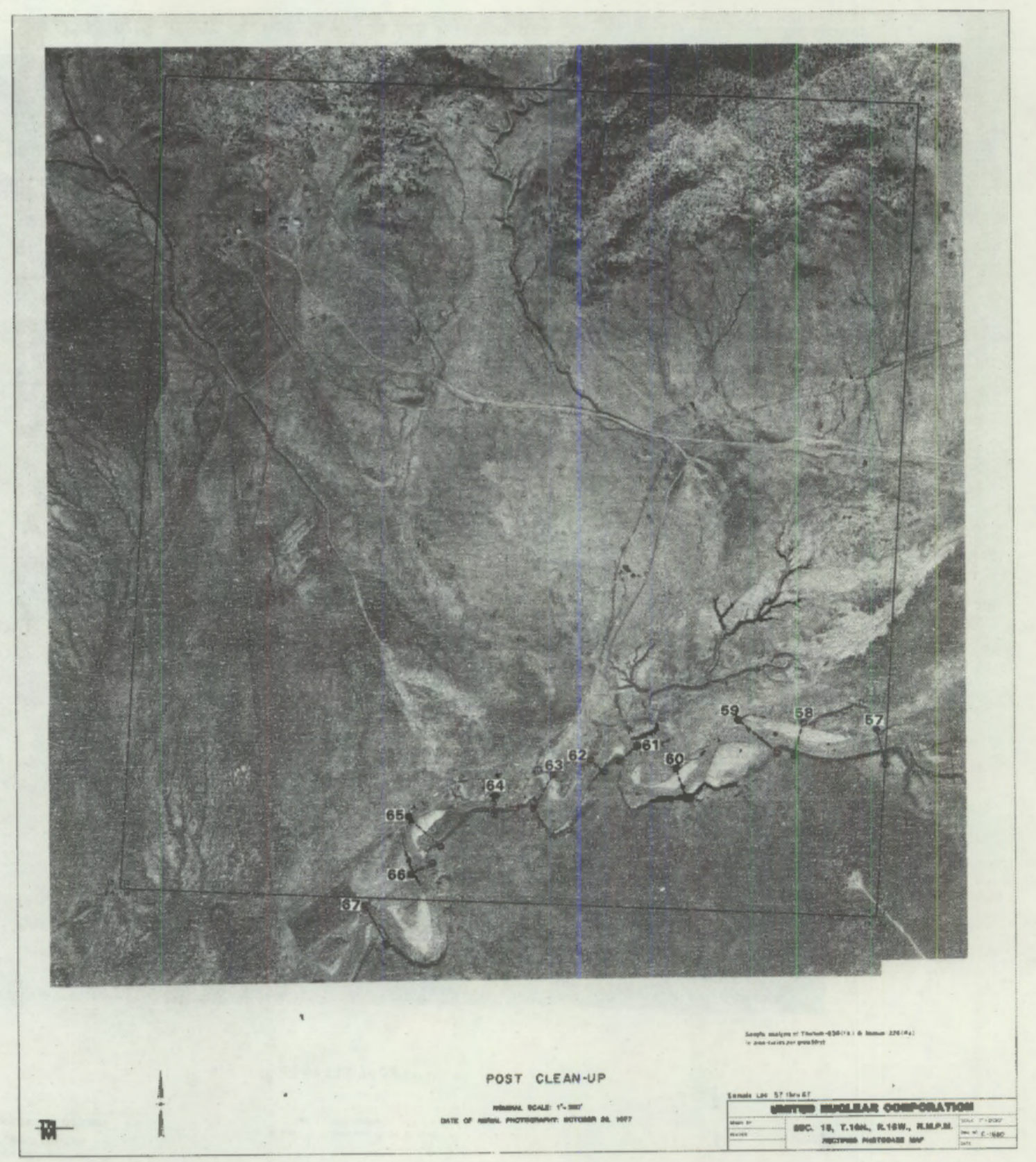

D. 8 


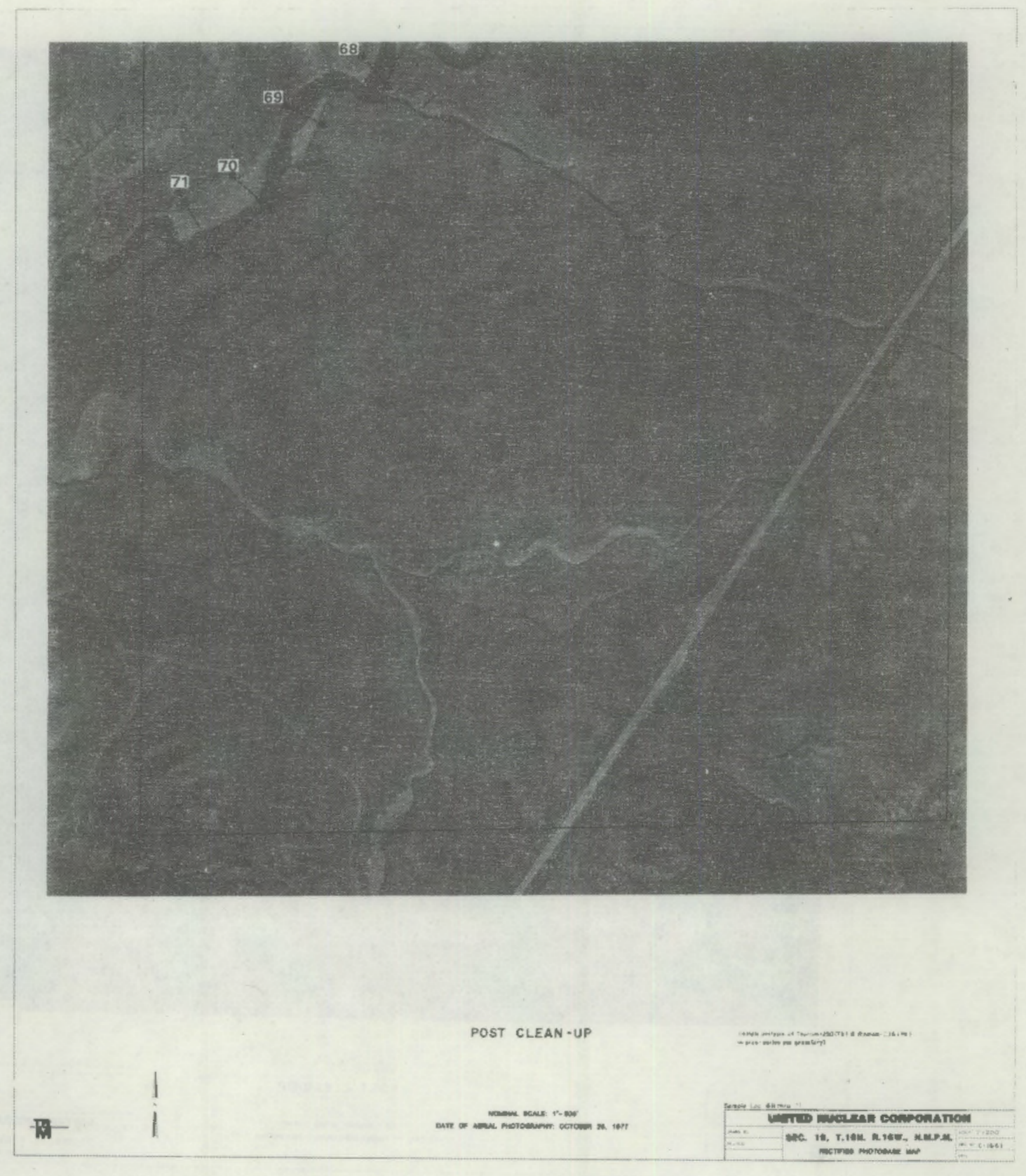

D. 9 


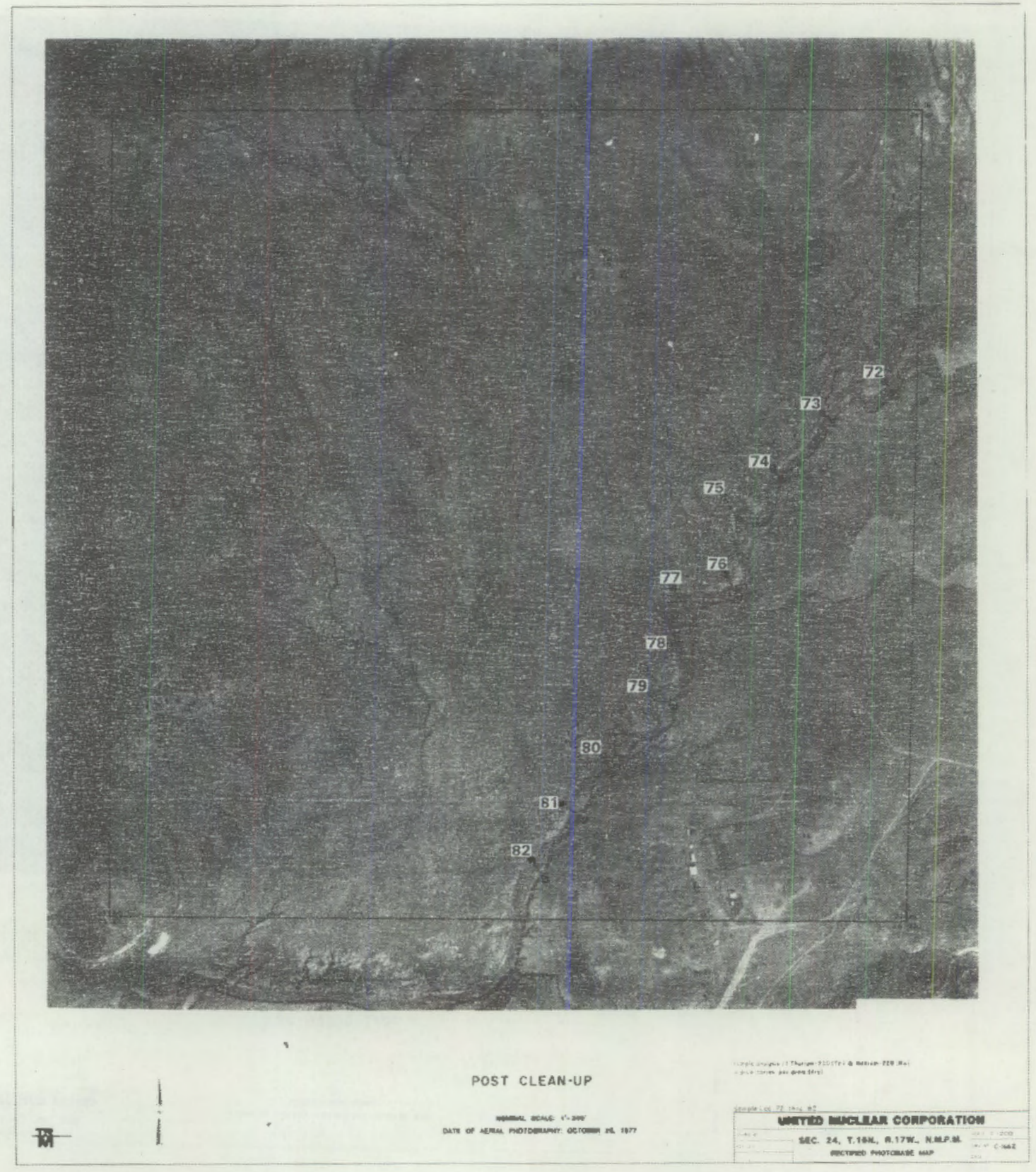

D. 10 


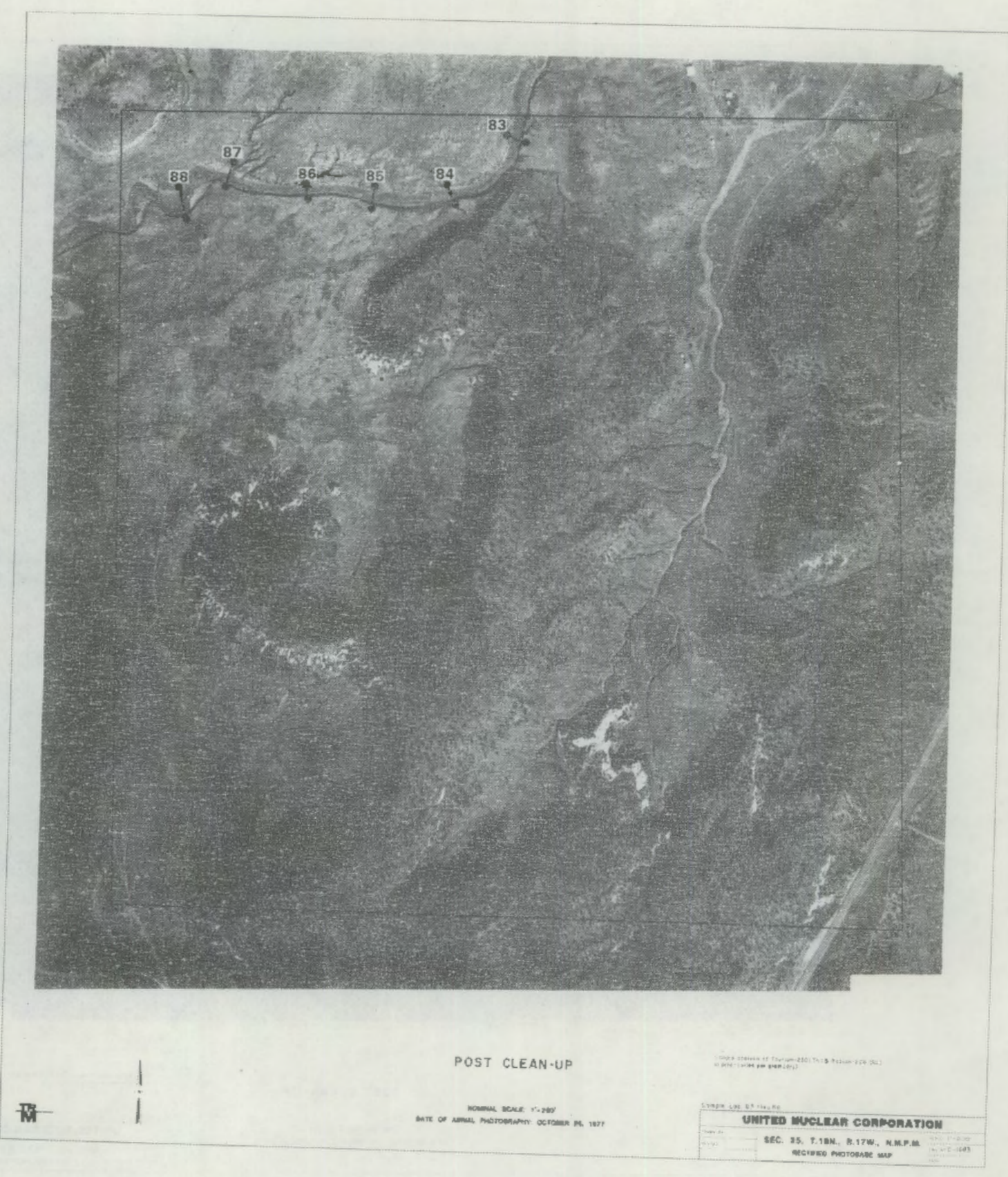

0.11 


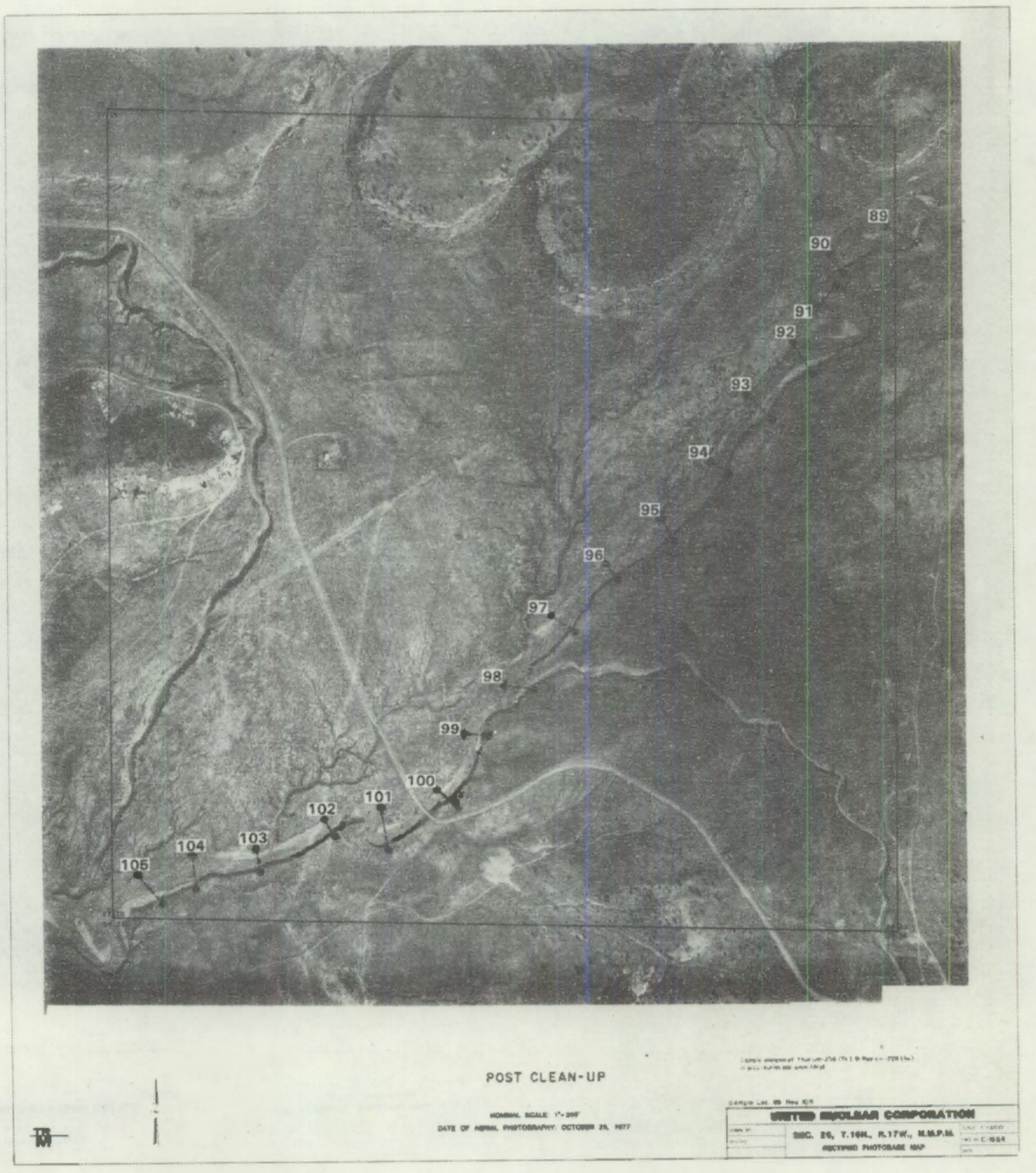

D. 12 


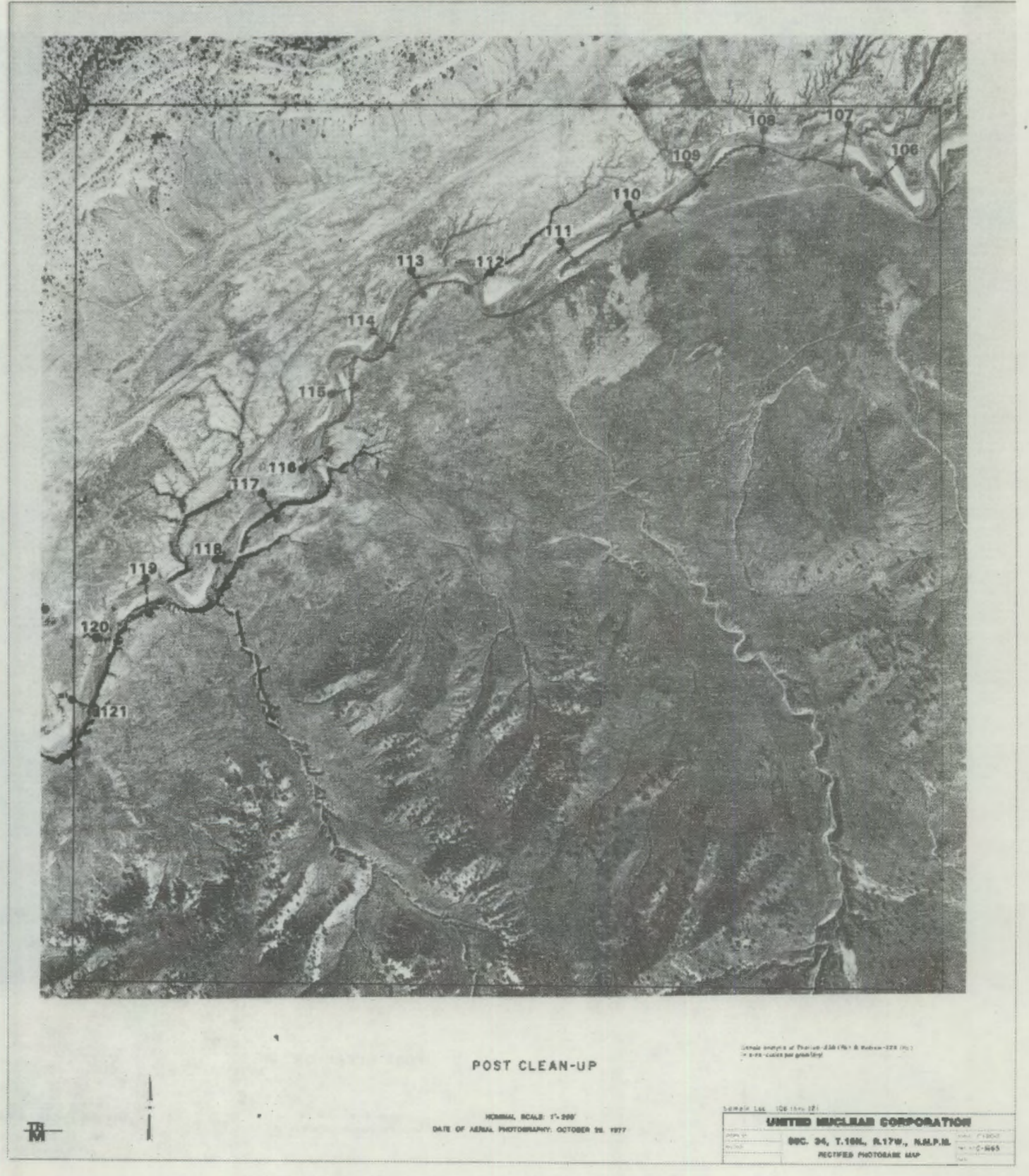




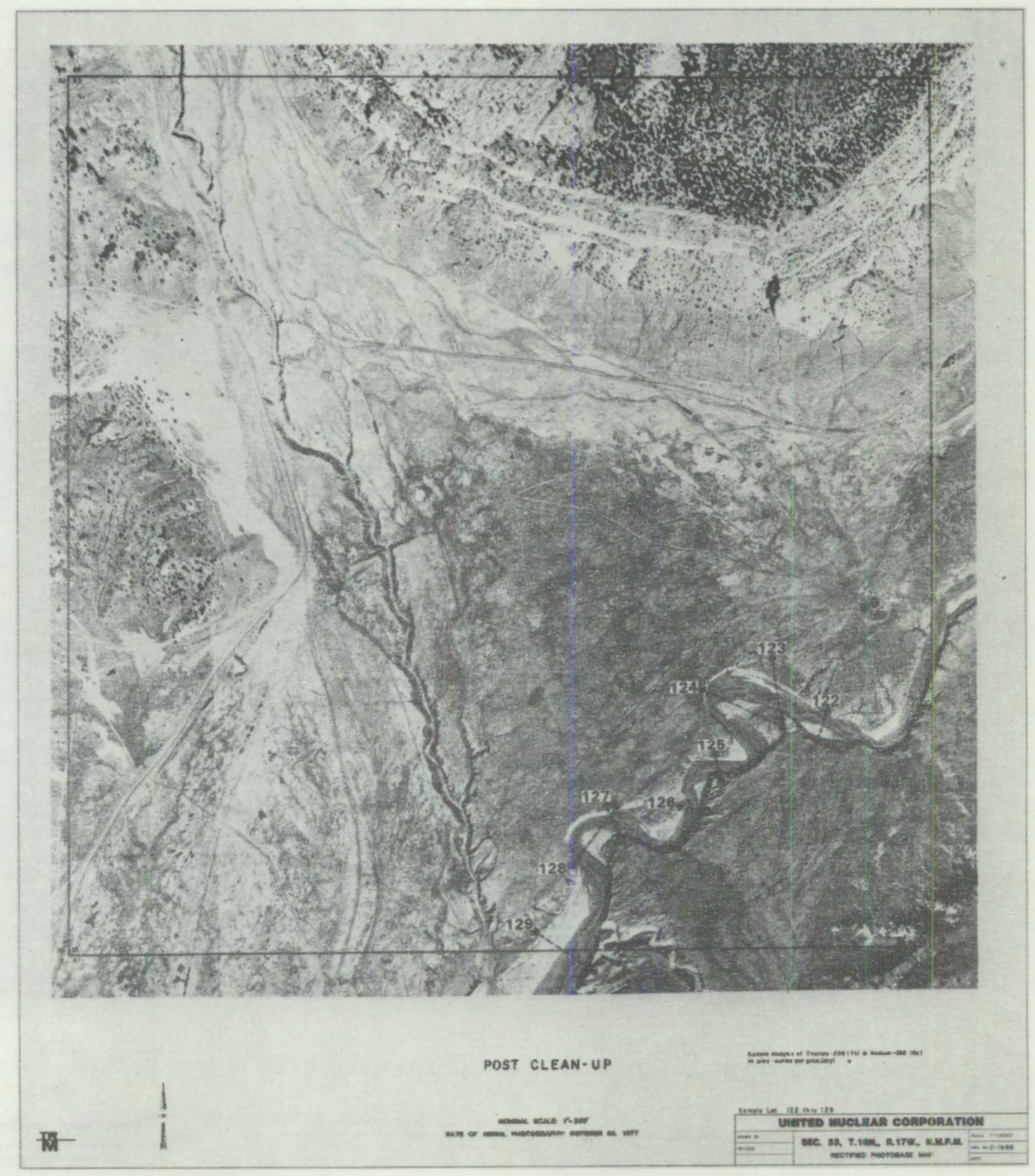

D. 14 


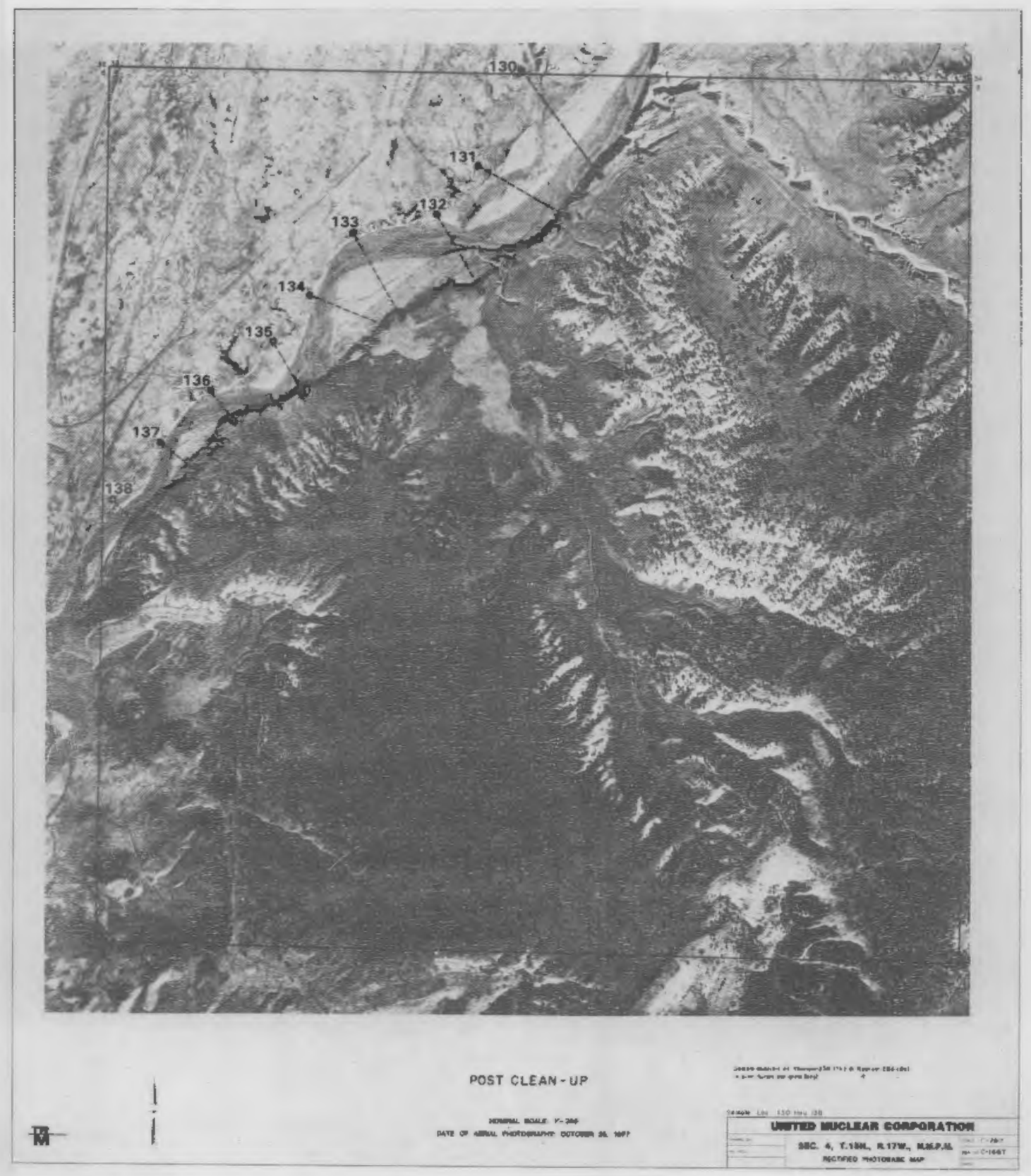




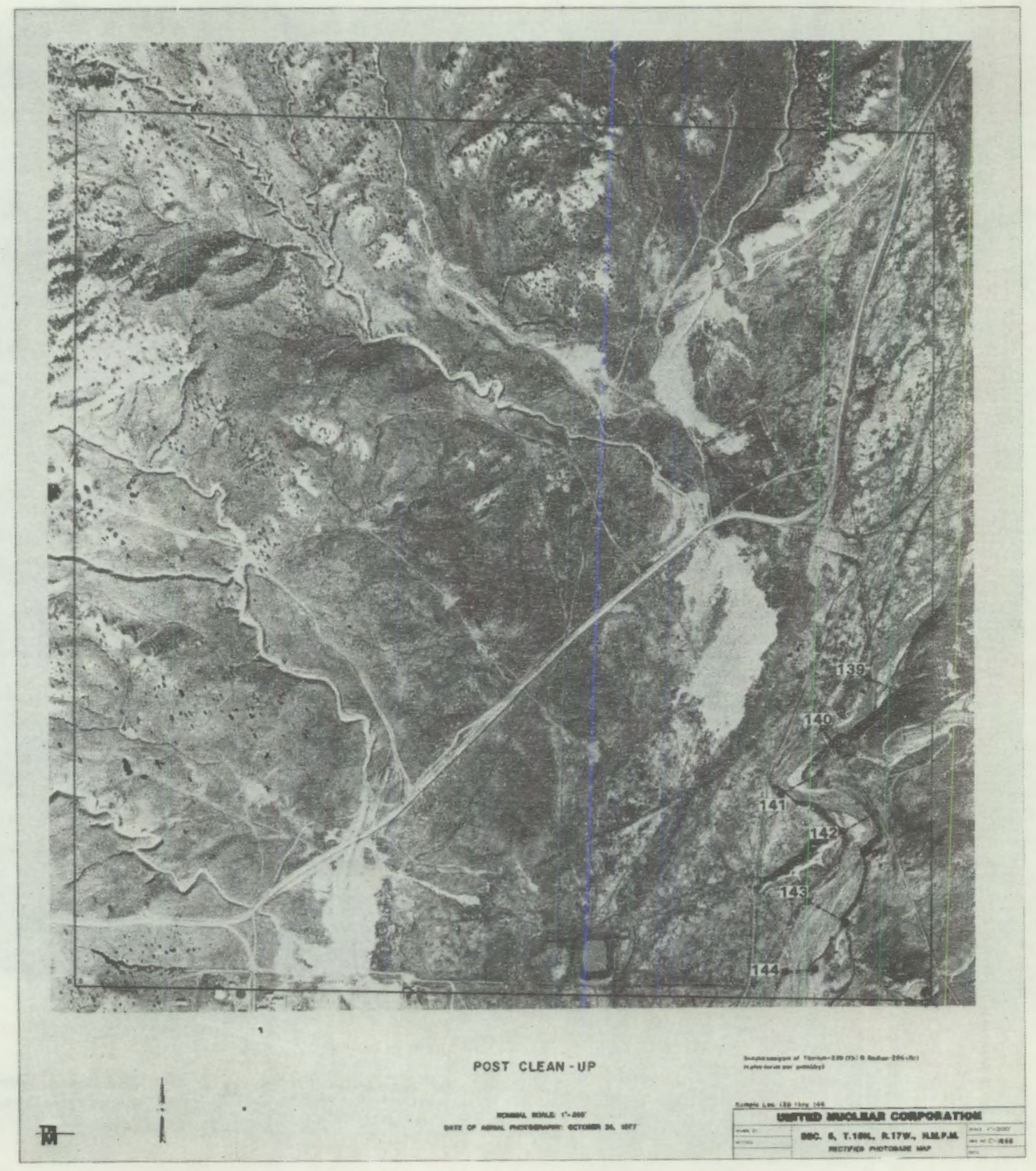




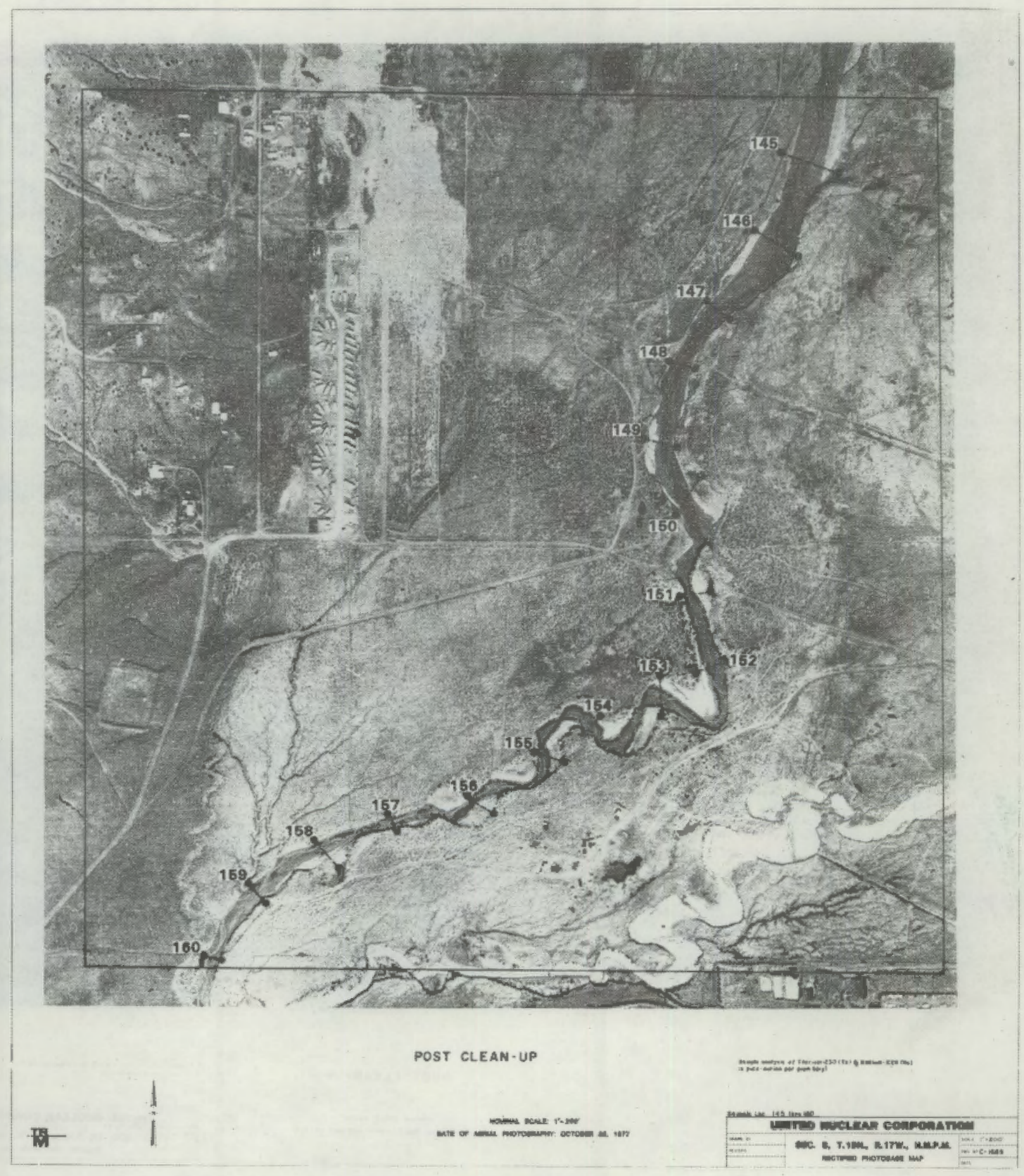




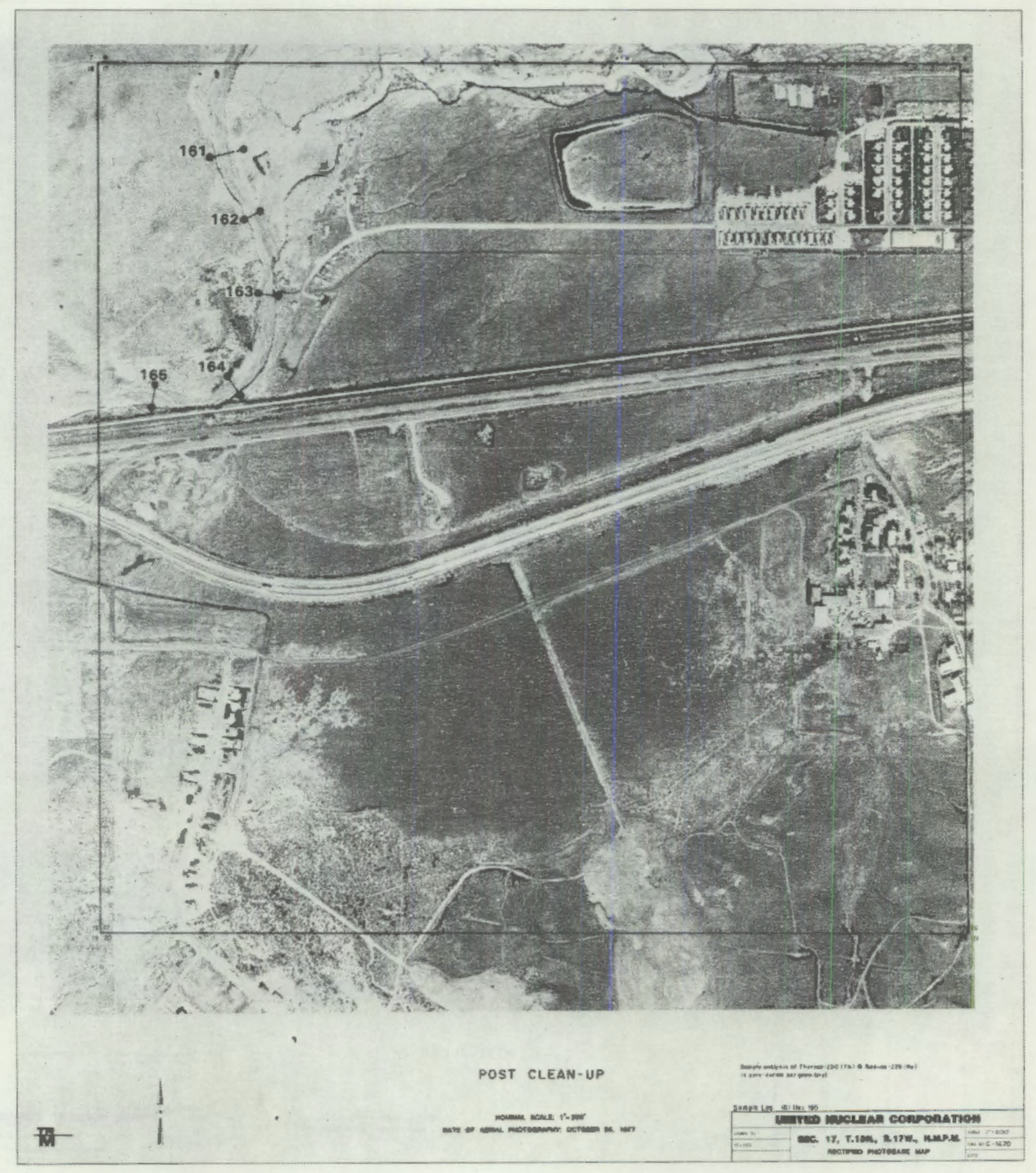

D. 18 


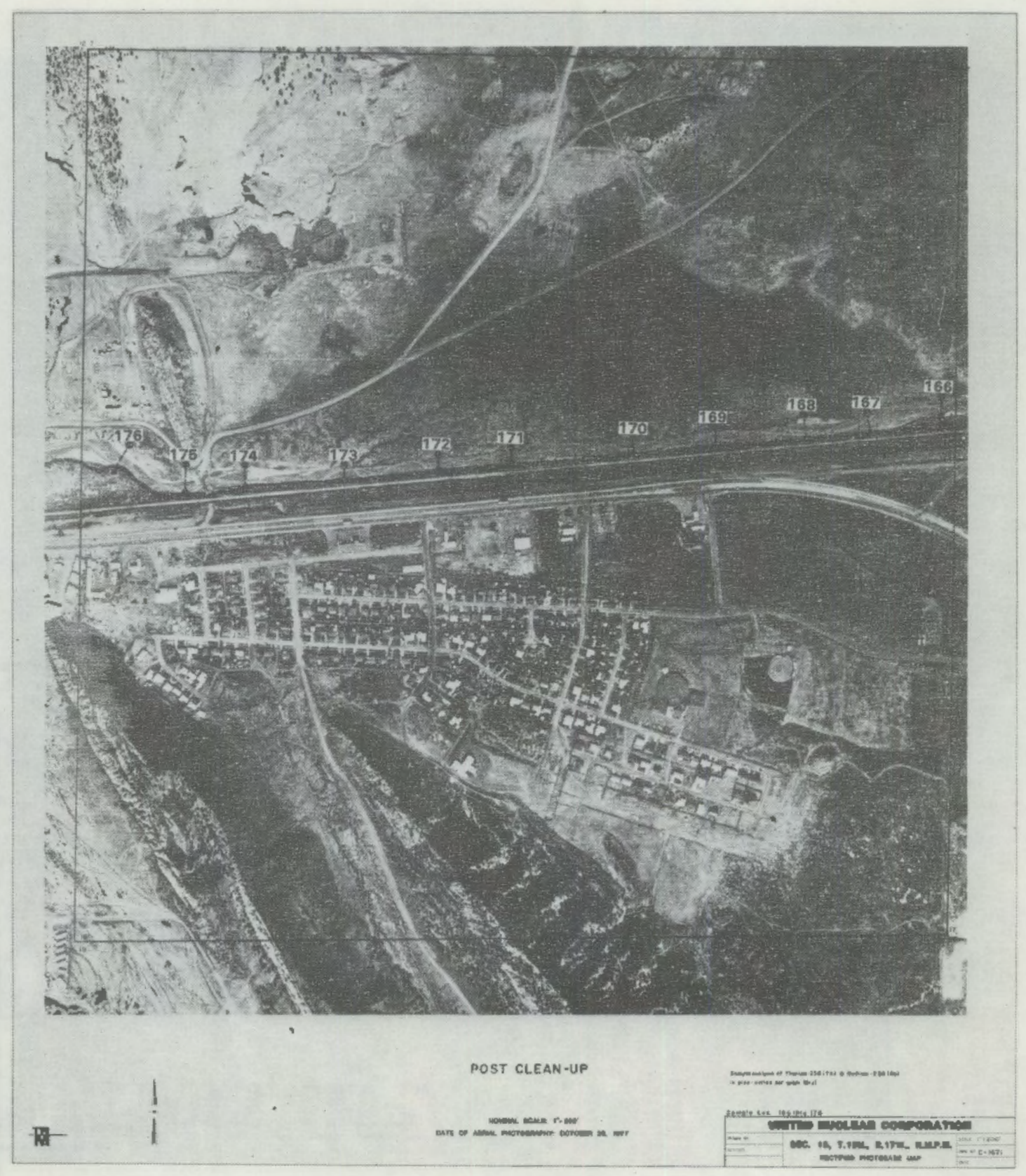

D. 19 


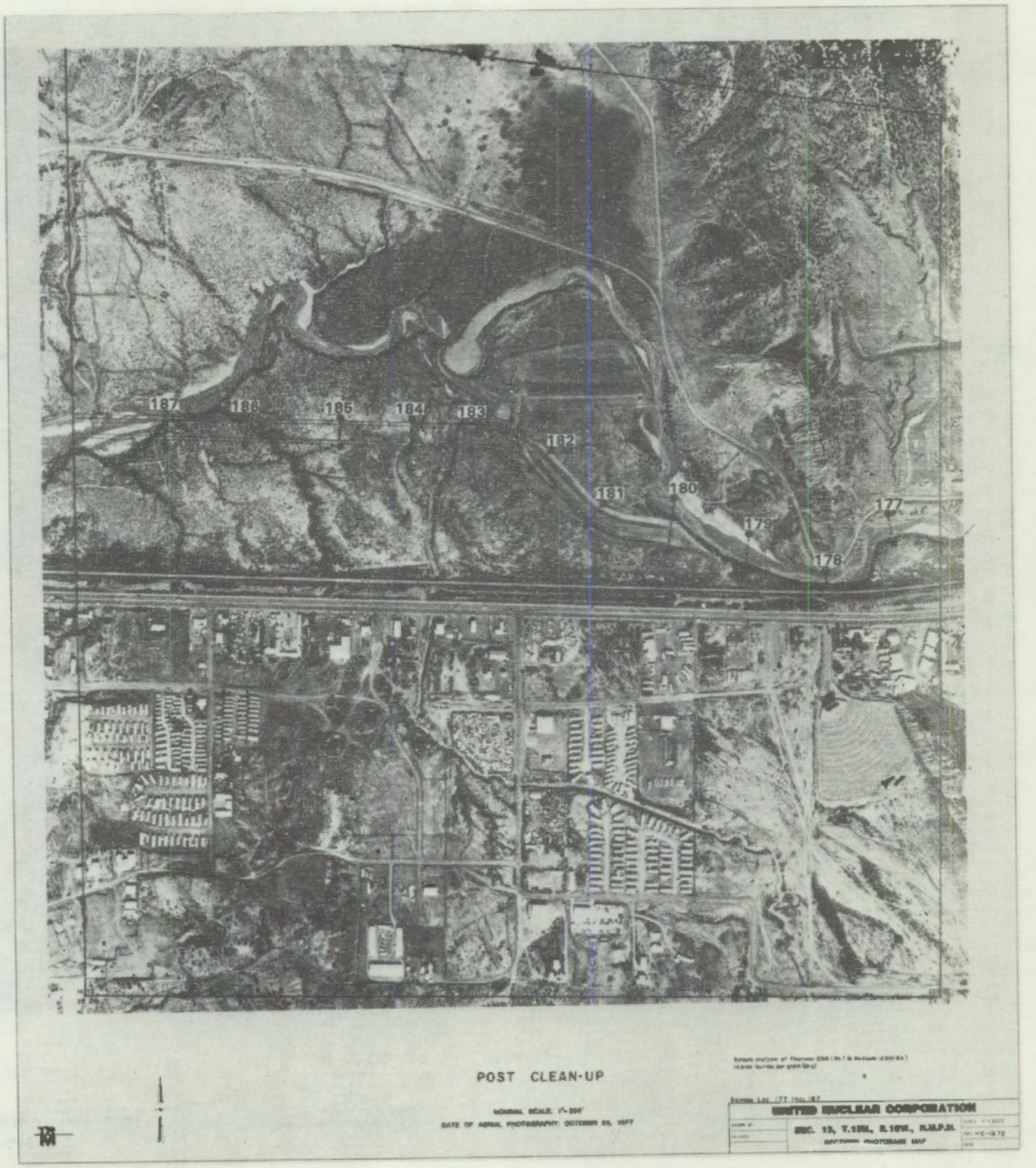




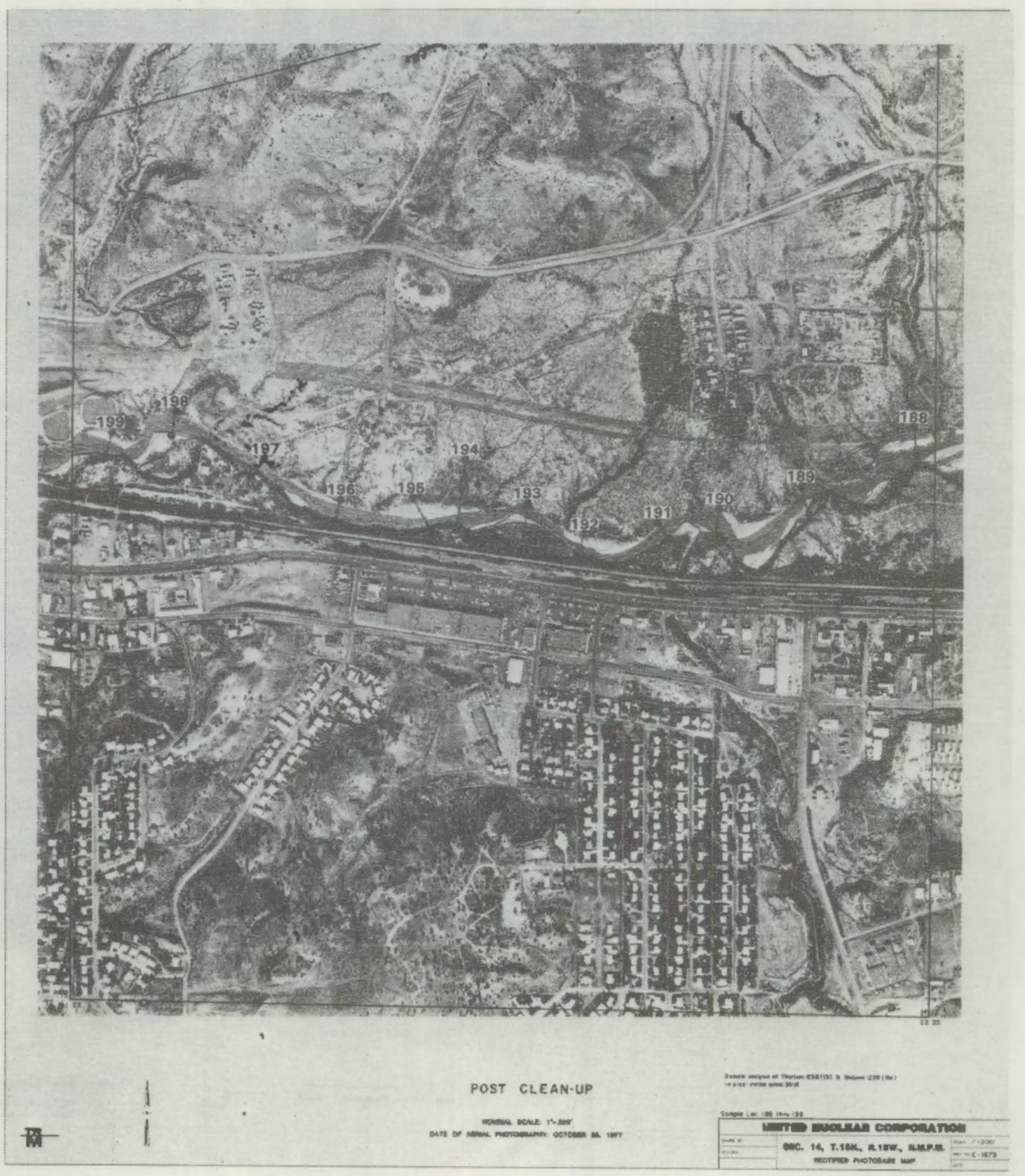




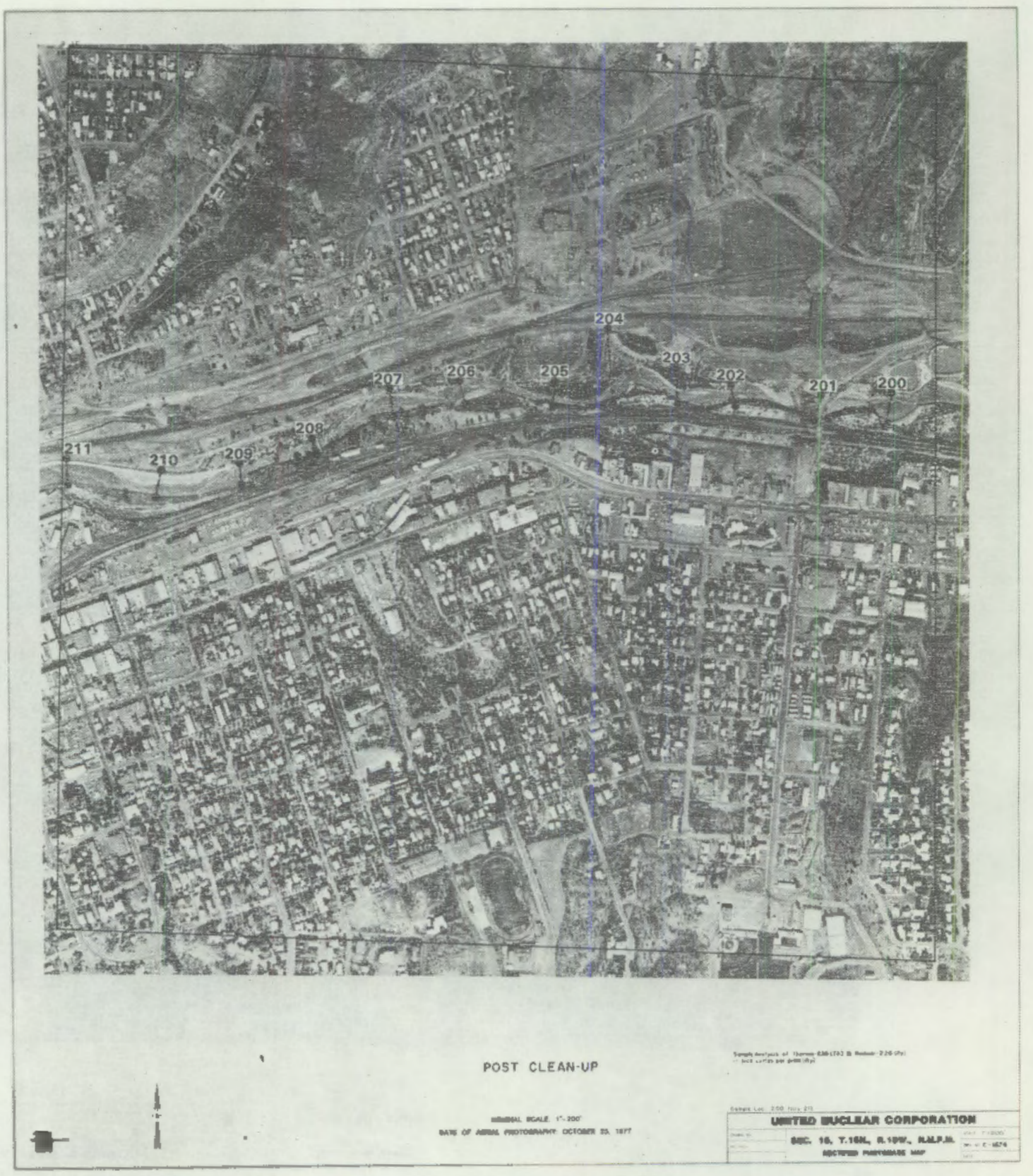




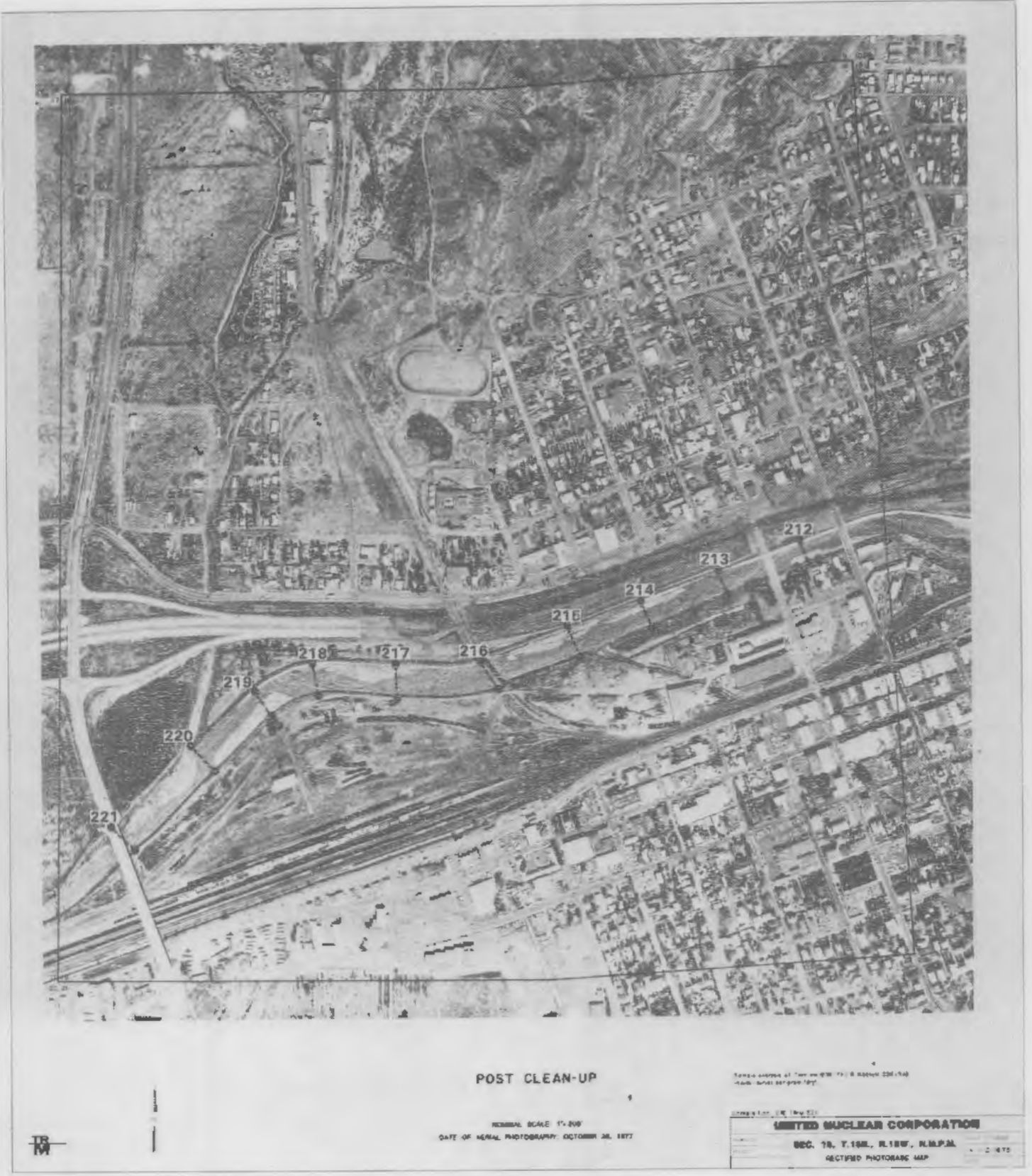




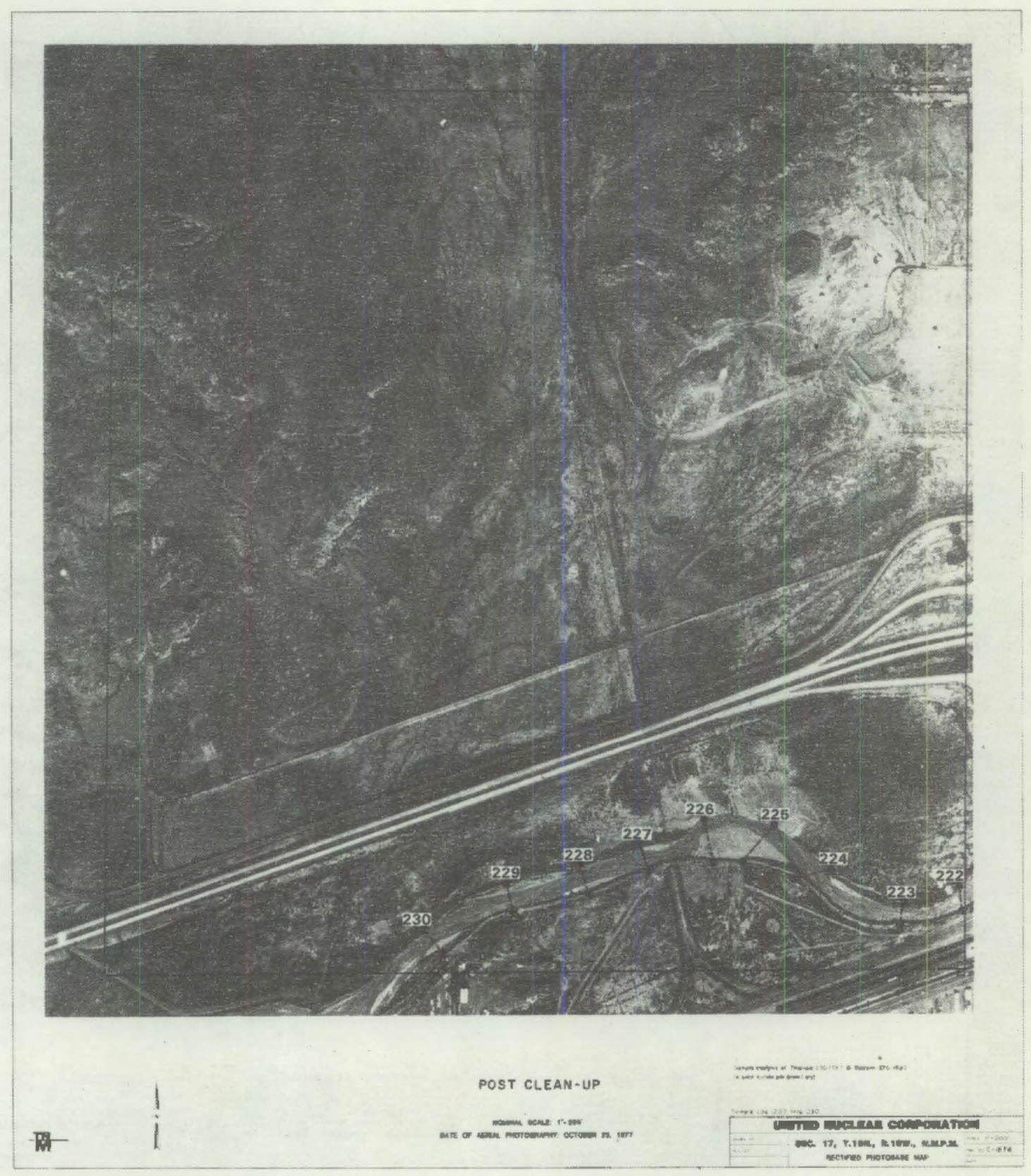




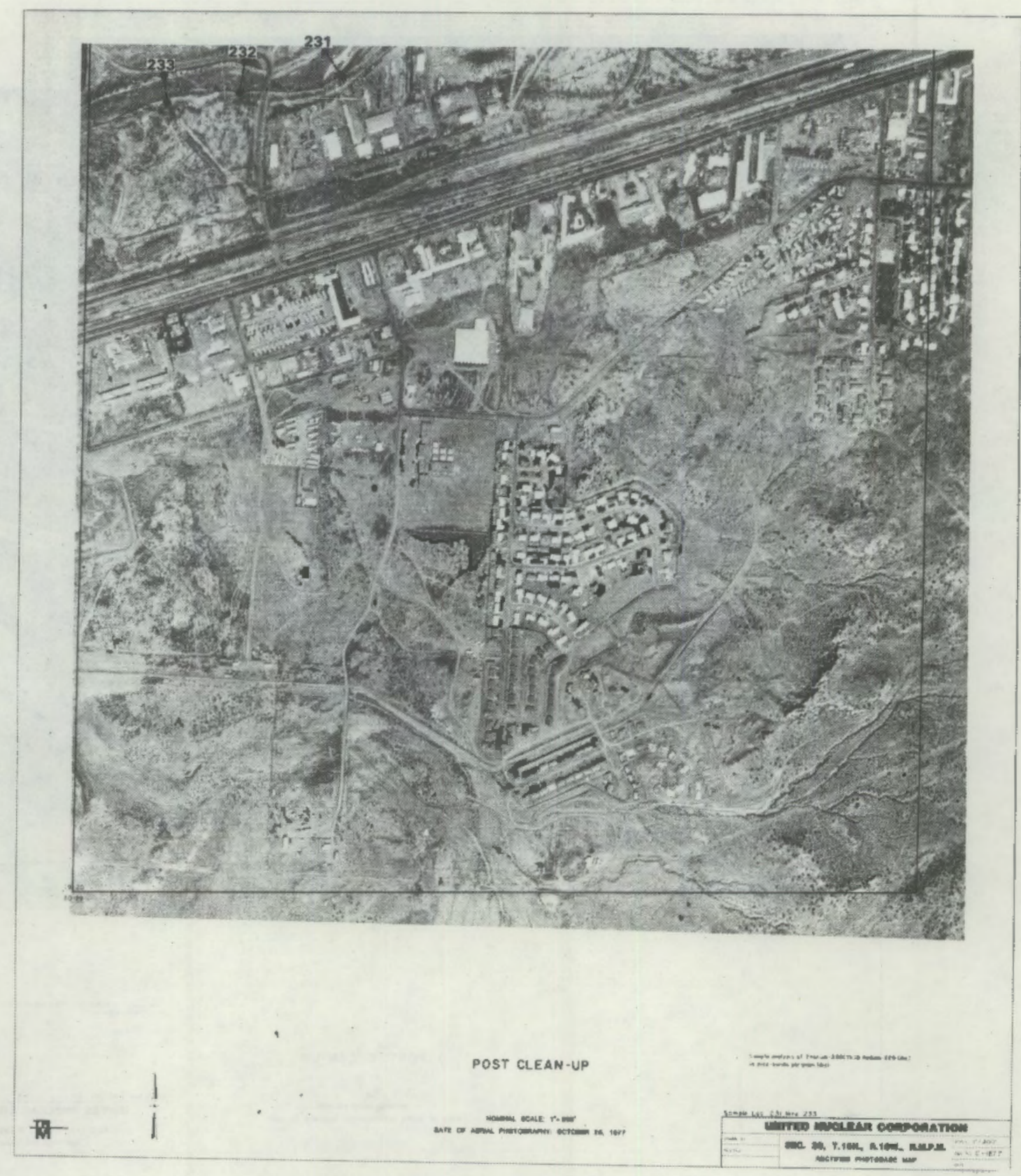




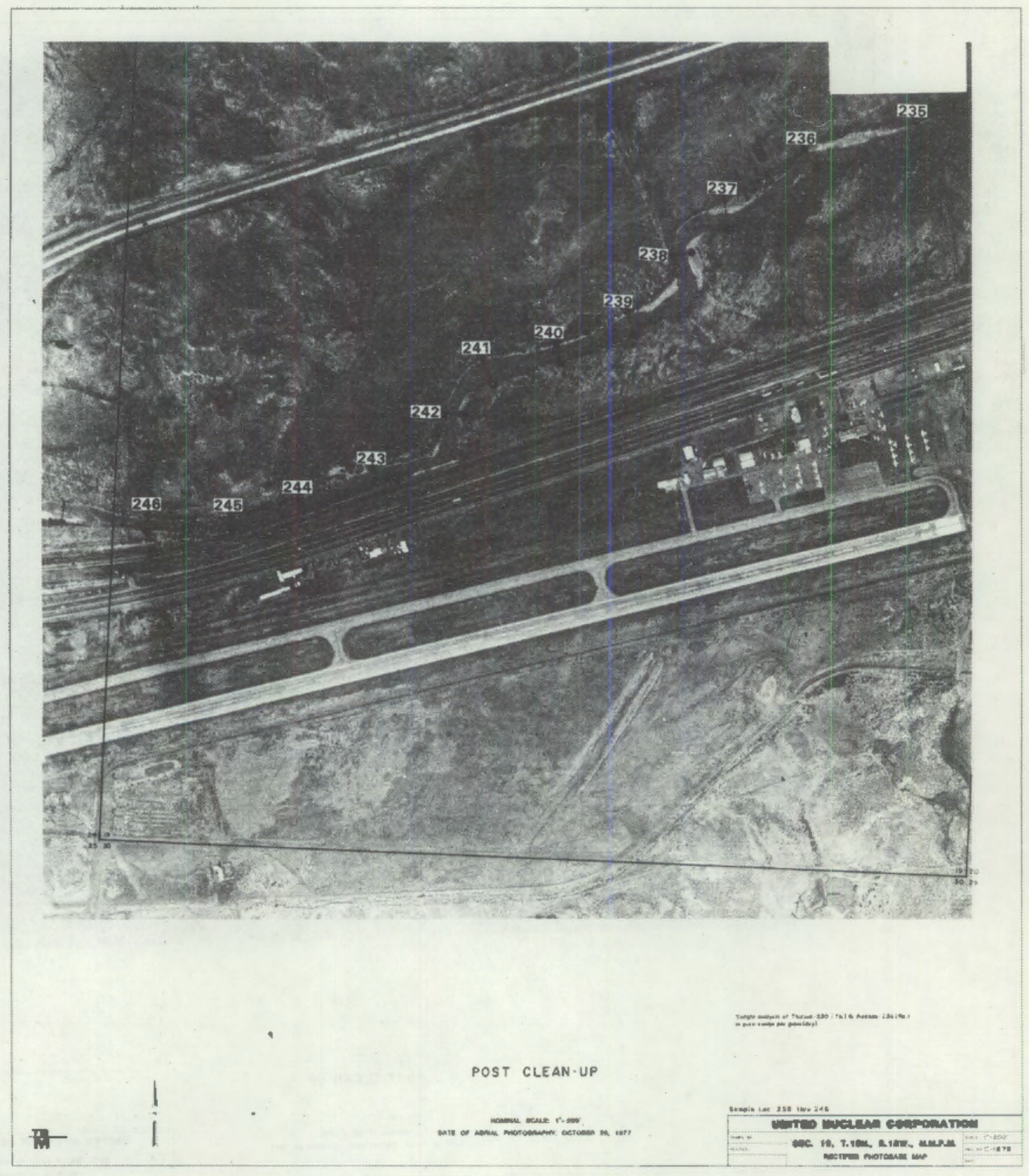




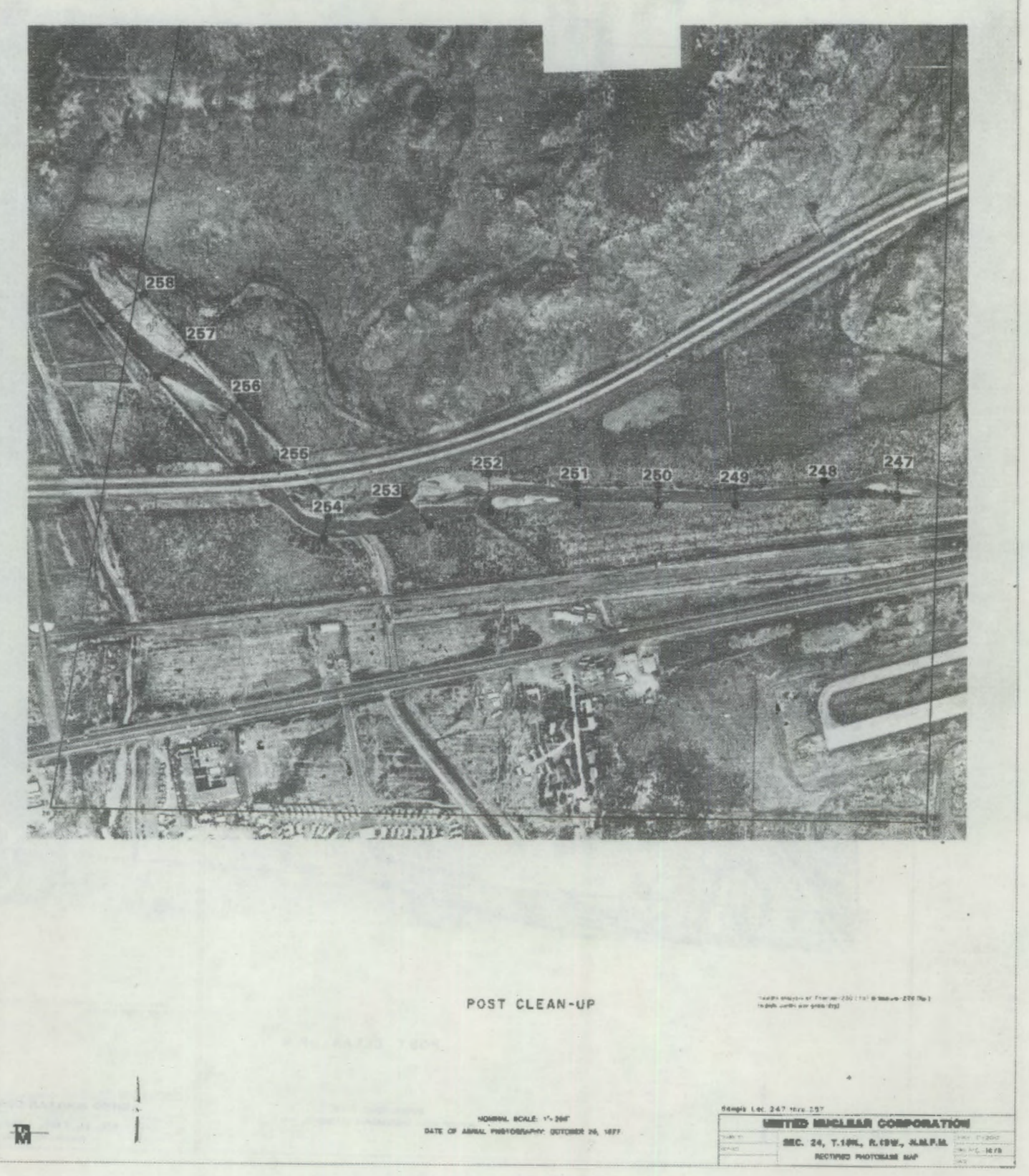




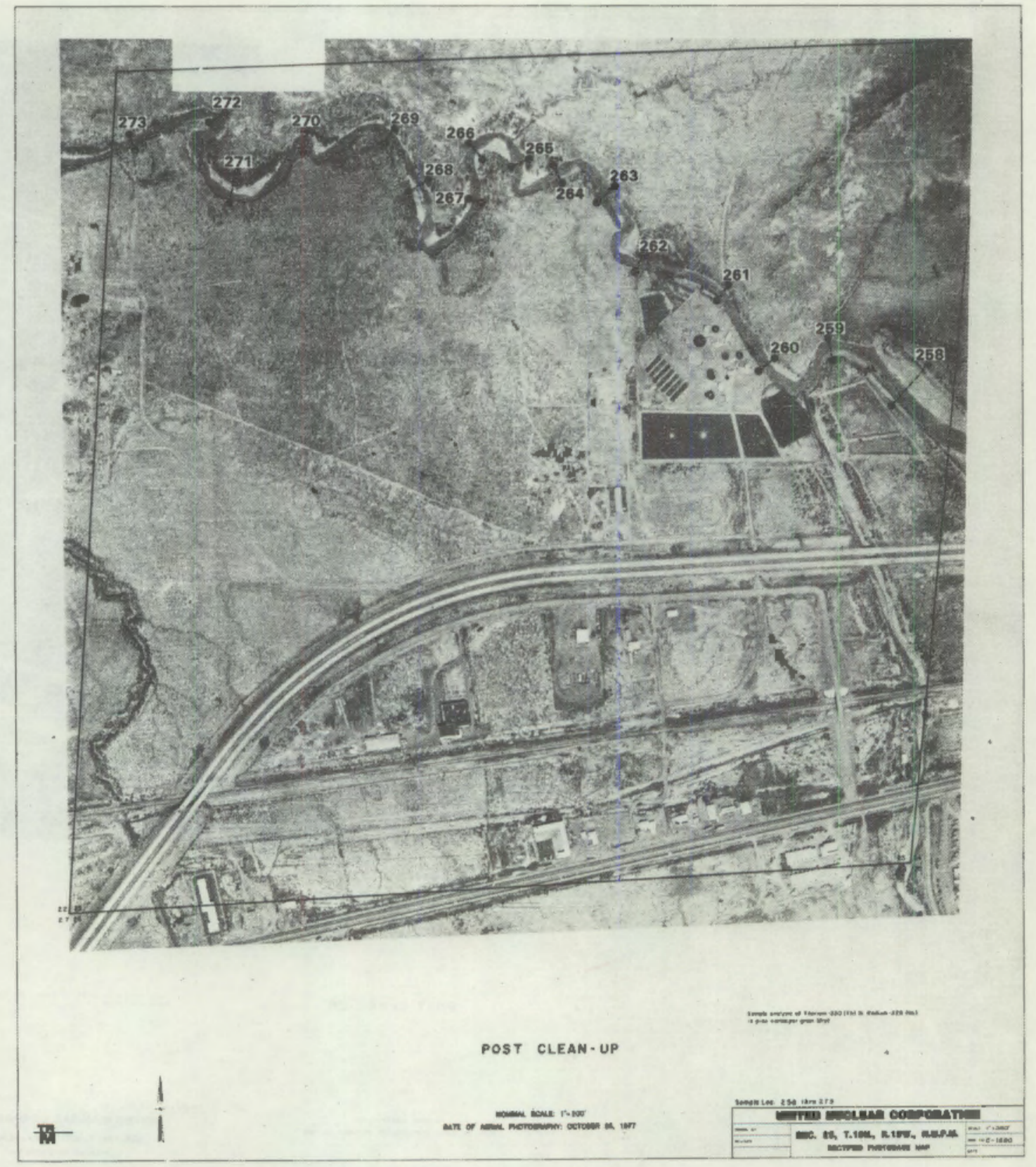




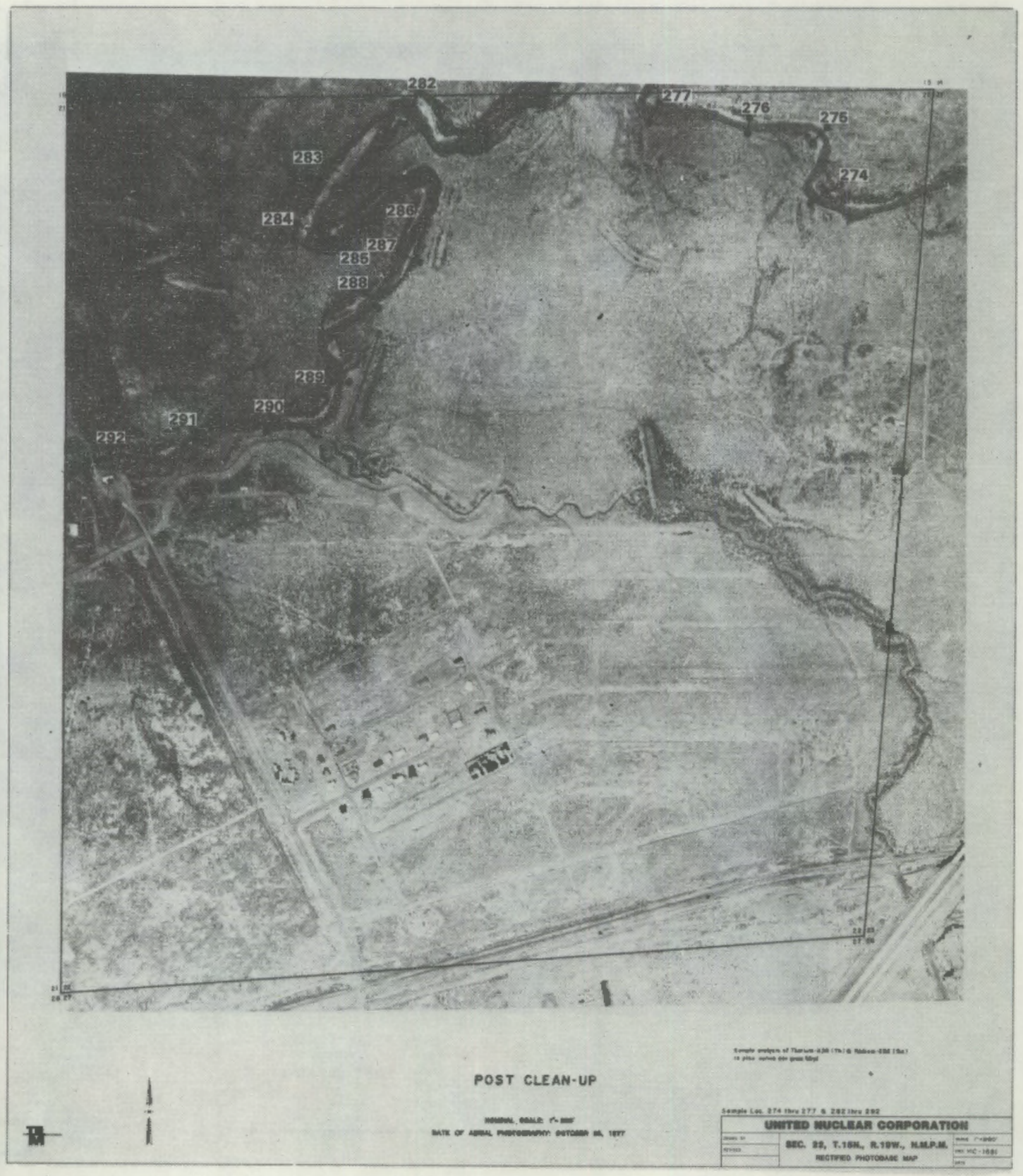




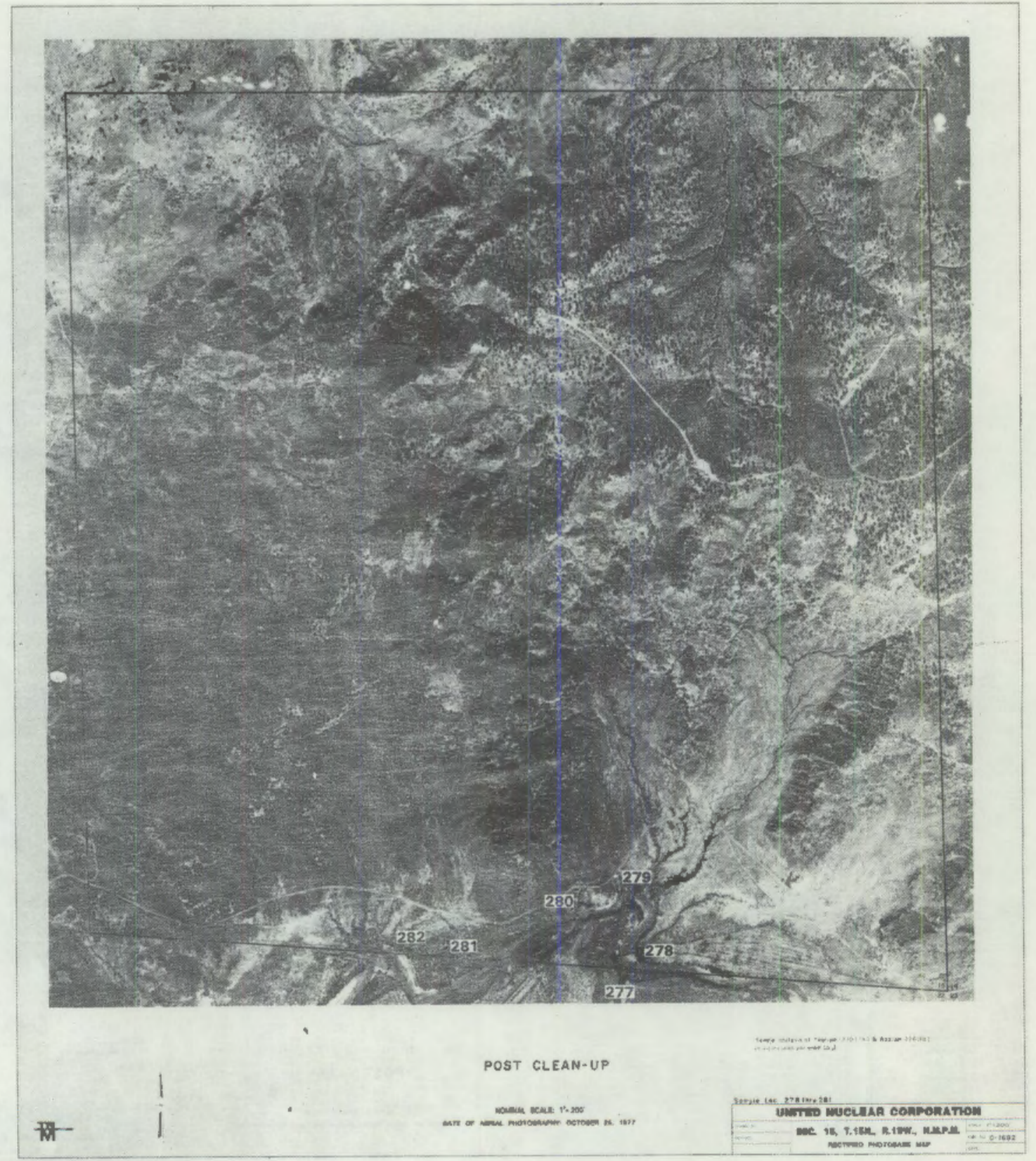




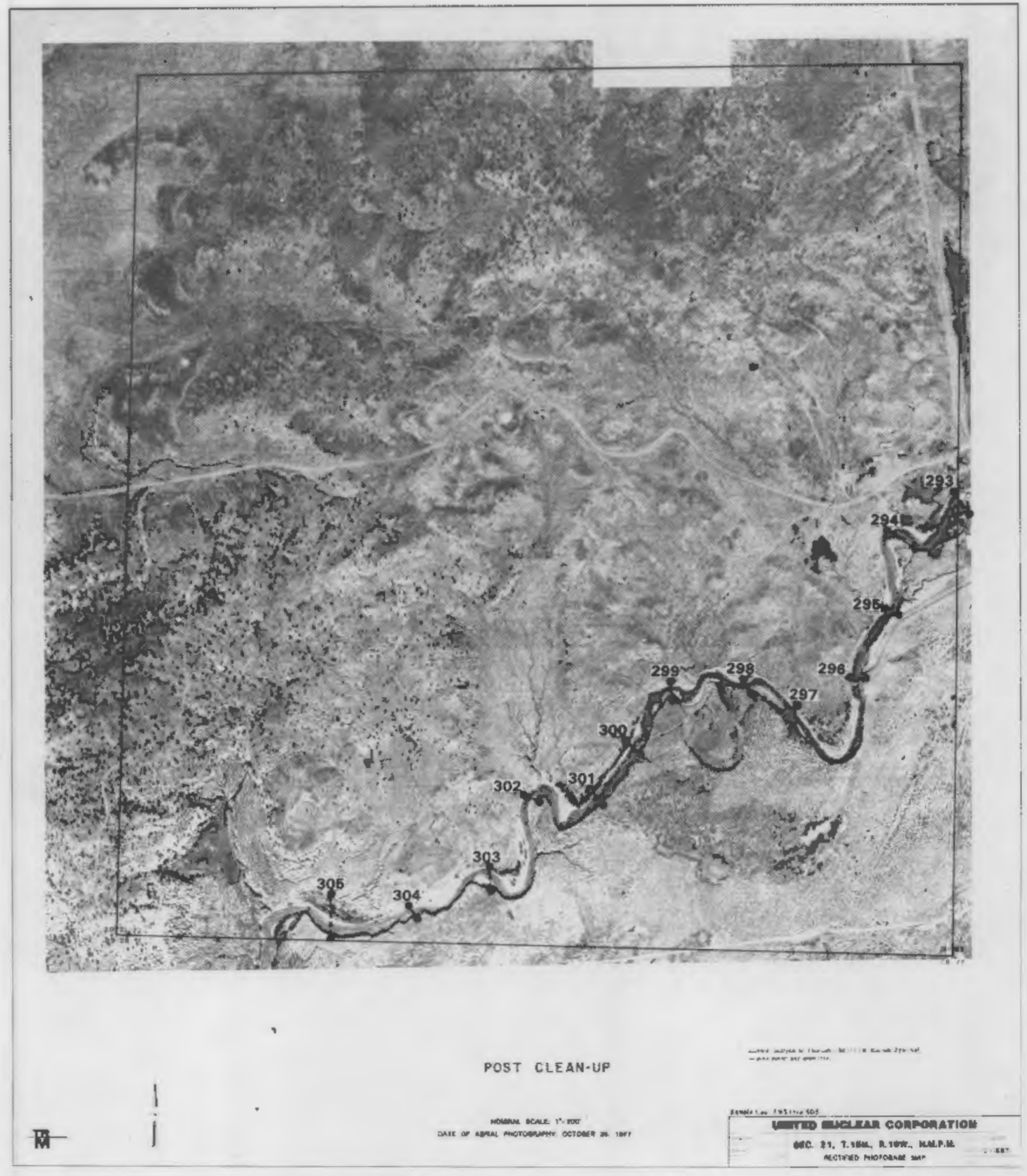



APPENDIX E

DATA FOR SITE VARIABILITY STUDIES 
APPENDIX E

\section{DATA FOR SITE VARIABILITY STUDIES}

\section{KEY TO APPENDIX E}

Level: Coricentration in $\rho \mathrm{C} i / \mathrm{g}$

Error: Counting Error.

TABLE E.1. Variability Study No. 1

\begin{tabular}{|c|c|c|c|c|c|c|c|c|}
\hline \multirow{2}{*}{$\begin{array}{l}\text { Sample } \\
\text { Number }\end{array}$} & \multicolumn{2}{|c|}{ Thor ium } & \multicolumn{2}{|c|}{ Radium } & \multicolumn{2}{|c|}{ Lead } & \multicolumn{2}{|c|}{ Uranium } \\
\hline & Level & Error & Leve] & Error & [eve] & Error & Leve] & Error \\
\hline 01 & -3.5 & 13 & - & - & 2.7 & 1.5 & 0.0 & 1.0 \\
\hline 02 & 17 & 8.6 & - & - & 1.1 & 0.93 & 0.0 & 0.88 \\
\hline 03 & 27 & 15 & - & - & 5.6 & 1.6 & 1.4 & 0.80 \\
\hline 04 & -10 & 9.1 & - & - & 1.0 & 0.95 & 0.90 & 1.0 \\
\hline 05 & 64 & 17 & 2.4 & 0.50 & 7.3 & 2.0 & 0.61 & 1.1 \\
\hline 06 & 72 & 15 & 1.7 & 0.46 & 8.3 & 1.5 & 0.0 & 0.98 \\
\hline 07 & 97 & 20 & - & - & 10 & 2.4 & -0.20 & 1.2 \\
\hline 08 & 51 & 13 & - & - & 6.5 & 1.4 & -1.3 & 1.1 \\
\hline 09 & 170 & 20 & 2.6 & 0.34 & 16 & 2.2 & 0.54 & 1.4 \\
\hline & 74 & 17 & - & - & 4.6 & 2.0 & 0.41 & 0.91 \\
\hline 010 & 71 & 17 & 1.3 & 0.45 & 2.7 & 1.8 & 3.1 & 1.2 \\
\hline 011 & 48 & 13 & - & - & 3.3 & 1.3 & -0.30 & 0.36 \\
\hline 012 & 30 & 15 & - & - & 5.8 & 1.6 & 1.0 & 0.84 \\
\hline 013 & 30 & 14 & - & - & 2.3 & 1.5 & -1.2 & 1.2 \\
\hline 014 & 11 & 12 & - & - & 4.1 & 1.2 & -0.54 & 0.94 \\
\hline 010 & 58 & 13 & - & - & 4.4 & 1.2 & 0.0 & 0.90 \\
\hline 016 & 71 & 12 & - & - & 8.7 & 1.3 & 0.36 & 1.1 \\
\hline 017 & 88 & 16 & - & - & 0.21 & 1.9 & 1.0 & 1.0 \\
\hline 018 & 81 & 16 & 6.1 & 1.8 & 7.1 & 1.8 & -1.6 & 1.0 \\
\hline 019 & 48 & Is & 7.4 & 2.0 & 5.1 & 1.3 & 0.54 & 1.0 \\
\hline 020 & $y 2$ & 16 & 2.1 & 4.8 & 7.7 & 1.5 & 0.72 & 1.0 \\
\hline 0302 & b. 3 & 13 & 2.0 & 1.6 & 4.4 & 1.5 & -1.4 & 0.98 \\
\hline & 25 & 13 & - & - & 4.0 & 1.6 & 0.61 & 0.98 \\
\hline & 19 & 2.8 & 2.7 & 0.37 & 2.2 & 0.35 & -0.15 & 0.22 \\
\hline & 44 & 14 & - & - & 1.3 & 1.4 & 0.20 & 0.80 \\
\hline
\end{tabular}

E.1 
TABLE E.1. (conta)

Variābility Scudy No. 1

\begin{tabular}{|c|c|c|c|c|c|c|c|c|}
\hline \multirow{2}{*}{$\begin{array}{l}\text { Sample } \\
\text { Number }\end{array}$} & \multicolumn{2}{|c|}{ Thorium } & \multicolumn{2}{|c|}{ Radium } & \multicolumn{2}{|c|}{ Lead } & \multicolumn{2}{|c|}{ Uranium } \\
\hline & Eevel & Error & Level & Error & Level & Error & Level & Error \\
\hline $\begin{array}{l}0106 \\
0206 \\
0301 \\
0303\end{array}$ & $\begin{array}{l}-1.2 \\
23 \\
22 \\
30 \\
19\end{array}$ & $\begin{array}{c}7.5 \\
13 \\
9.0 \\
14 \\
11\end{array}$ & $\begin{array}{c}- \\
1.6 \\
3.8 \\
- \\
-\end{array}$ & $\begin{array}{l}- \\
0.27 \\
1.5 \\
- \\
-\end{array}$ & $\begin{array}{l}3.0 \\
4.2 \\
2.5 \\
4.0 \\
1.3\end{array}$ & $\begin{array}{l}1.0 \\
1.7 \\
1.0 \\
1.6 \\
1.7\end{array}$ & $\begin{array}{c}0.0 \\
-1.0 \\
0.19 \\
0.41 \\
0.20\end{array}$ & $\begin{array}{l}0.88 \\
1.0 \\
0.90 \\
1.0 \\
1.1\end{array}$ \\
\hline $\begin{array}{l}0304 \\
0305 \\
0306 \\
0307\end{array}$ & $\begin{array}{l}25 \\
3.7 \\
-3.7 \\
21\end{array}$ & $\begin{array}{l}11 \\
8.7 \\
8.7 \\
9.2\end{array}$ & $\begin{array}{l}- \\
- \\
-\end{array}$ & $\begin{array}{l}- \\
- \\
-\end{array}$ & $\begin{array}{l}2.1 \\
2.8 \\
2.7 \\
2.6\end{array}$ & $\begin{array}{l}1.5 \\
1.1 \\
0.90 \\
1.0\end{array}$ & $\begin{array}{l}0.0 \\
1.4 \\
0.0 \\
0.18\end{array}$ & $\begin{array}{l}2.2 \\
0.92 \\
0.77 \\
0.87\end{array}$ \\
\hline $\begin{array}{l}0308 \\
0401 \\
04 D 2 \\
0403\end{array}$ & $\begin{array}{l}0.0 \\
16 \\
10 \\
8.9\end{array}$ & $\begin{array}{r}9.1 \\
9.8 \\
8.8 \\
11\end{array}$ & $\begin{array}{l}- \\
- \\
-\end{array}$ & $\begin{array}{l}- \\
- \\
-\end{array}$ & $\begin{array}{l}1.6 \\
4.3 \\
4.1 \\
2.3\end{array}$ & $\begin{array}{l}1.0 \\
1.2 \\
1.0 \\
1.5\end{array}$ & $\begin{array}{r}0.0 \\
-1.1 \\
1.6 \\
-1.0\end{array}$ & $\begin{array}{l}0.92 \\
0.99 \\
0.94 \\
0.94\end{array}$ \\
\hline $\begin{array}{l}0404 \\
0405 \\
0406\end{array}$ & $\begin{array}{l}55 \\
23 \\
23 \\
35\end{array}$ & $\begin{array}{l}15 \\
13 \\
13 \\
13\end{array}$ & $\begin{array}{l}- \\
- \\
-\end{array}$ & $\begin{array}{l}- \\
- \\
-\end{array}$ & $\begin{array}{l}4.8 \\
1.0 \\
4.4 \\
4.2\end{array}$ & $\begin{array}{l}1.9 \\
1.7 \\
1.7 \\
1.7\end{array}$ & $\begin{array}{c}-1.0 \\
0.61 \\
-1.6 \\
0.61\end{array}$ & $\begin{array}{l}1.3 \\
1.1 \\
0.91 \\
0.84\end{array}$ \\
\hline $\begin{array}{l}0407 \\
0408 \\
0409\end{array}$ & $\begin{array}{l}23 \\
16 \\
23 \\
32\end{array}$ & $\begin{array}{c}13 \\
9.3 \\
12 \\
10\end{array}$ & $\begin{array}{l}0.75 \\
- \\
- \\
-\end{array}$ & $\begin{array}{l}0.26 \\
- \\
- \\
-\end{array}$ & $\begin{array}{l}0.63 \\
3.2 \\
3.1 \\
1.8\end{array}$ & $\begin{array}{l}1.6 \\
1.1 \\
1.4 \\
1.1\end{array}$ & $\begin{array}{c}0.41 \\
-0.36 \\
1.0 \\
0.54\end{array}$ & $\begin{array}{l}0.87 \\
0.99 \\
0.84 \\
0.94\end{array}$ \\
\hline $\begin{array}{l}04010 \\
0501 \\
0503 \\
0509\end{array}$ & $\begin{array}{r}30 \\
18 \\
-14 \\
-15\end{array}$ & $\begin{array}{l}12 \\
10 \\
12 \\
15\end{array}$ & $\begin{array}{l}- \\
- \\
-\end{array}$ & $\begin{array}{l}- \\
- \\
-\end{array}$ & $\begin{array}{l}0.21 \\
1.5 \\
2.5 \\
1.5\end{array}$ & $\begin{array}{l}1.2 \\
1.5 \\
1.6 \\
1.6\end{array}$ & $\begin{array}{r}0.0 \\
-1.2 \\
-1.2 \\
0.41\end{array}$ & $\begin{array}{l}1.2 \\
0.82 \\
1.1 \\
1.3\end{array}$ \\
\hline $\begin{array}{l}02 U 6 \\
03 \cup 1 \\
03 \cup 2 \\
03 U 3\end{array}$ & $\begin{array}{l}-8.8 \\
41 \\
57 \\
58\end{array}$ & $\begin{array}{l}13 \\
12 \\
16 \\
13\end{array}$ & $\begin{array}{l}- \\
- \\
- \\
0.54\end{array}$ & $\begin{array}{l}- \\
\overline{-} \\
0.42\end{array}$ & $\begin{array}{l}4.2 \\
4.4 \\
1.5 \\
5.7\end{array}$ & $\begin{array}{l}1.5 \\
1.2 \\
1.4 \\
1.2\end{array}$ & $\begin{array}{c}0.41 \\
-0.18 \\
0.0 \\
-0.54\end{array}$ & $\begin{array}{l}0.87 \\
0.83 \\
1.0 \\
0.97\end{array}$ \\
\hline $\begin{array}{l}03 \cup 4 \\
03 U 5 \\
03 U 6 \\
04 U 1\end{array}$ & $\begin{array}{l}21 \\
28 \\
52 \\
50\end{array}$ & $\begin{array}{c}9.0 \\
13 \\
12 \\
9.1\end{array}$ & $\begin{array}{l}- \\
1.7 \\
-\end{array}$ & $\begin{array}{l}- \\
1.6 \\
- \\
-\end{array}$ & $\begin{array}{l}1.2 \\
2.1 \\
2.9 \\
3.2\end{array}$ & $\begin{array}{l}0.95 \\
1.6 \\
1.0 \\
1.1\end{array}$ & $\begin{array}{l}0.54 \\
0.81 \\
0.18 \\
0.90\end{array}$ & $\begin{array}{l}0.74 \\
0.86 \\
0.90 \\
0.83\end{array}$ \\
\hline $\begin{array}{l}04 U 2 \\
04 \cup 3 \\
04 U 4 \\
05 \cup 3\end{array}$ & $\begin{array}{l}26 \\
42 \\
48 \\
7.1\end{array}$ & $\begin{array}{c}10 \\
12 \\
16 \\
9.7\end{array}$ & $\begin{array}{l}- \\
- \\
1.1 \\
4.0\end{array}$ & $\begin{array}{l}- \\
- \\
0.24 \\
1.7\end{array}$ & $\begin{array}{l}7.0 \\
4.1 \\
7.1 \\
0.42\end{array}$ & $\begin{array}{l}1.2 \\
1.0 \\
2.0 \\
1.3\end{array}$ & $\begin{array}{c}-1.8 \\
1.6 \\
2.5 \\
0.20\end{array}$ & $\begin{array}{l}1.0 \\
1.2 \\
1.1 \\
0.98\end{array}$ \\
\hline
\end{tabular}


IABLE E.2. Variability Study No. 2

\begin{tabular}{|c|c|c|c|c|c|c|c|c|}
\hline \multirow{2}{*}{$\begin{array}{l}\text { Sample } \\
\text { Number }\end{array}$} & \multicolumn{2}{|c|}{ Thorium } & \multicolumn{2}{|c|}{ Radium } & \multicolumn{2}{|c|}{ Lead } & \multicolumn{2}{|c|}{ Uranium } \\
\hline & Eevel & Error & Level & Error & Level & Error & Leve! & Error \\
\hline 01 & 34 & 15 & - & - & 5.8 & 2.0 & 1.0 & 1.3 \\
\hline 02 & 27 & 14 & - & - & 3.1 & 1.6 & 0.20 & 0.68 \\
\hline 03 & 27 & 11 & - & - & 3.3 & 1.0 & 0.54 & 0.74 \\
\hline 04 & 27 & 7 & 1.1 & 0.9 & 3.3 & 0.7 & 1.1 & 0.7 \\
\hline $02 \cup 6$ & 37 & 15 & - & - & 5.4 & 1.8 & -1.6 & 1.2 \\
\hline 0301 & 49 & 12 & - & - & 3.0 & 1.2 & 1.4 & 0.99 \\
\hline 0302 & 14 & 13 & - & - & 4.4 & 1.6 & 0.41 & 1.2 \\
\hline $03 \cup 3$ & 28 & 9.7 & - & - & 4.0 & 1.1 & 2.0 & 1.0 \\
\hline $03 \cup 4$ & 32 & 10 & - & - & 2.3 & 1.2 & -1.6 & 1.0 \\
\hline 0305 & 11 & 8.5 & - & - & 2.2 & 1.0 & -2.2 & 0.81 \\
\hline 0346 & 15 & 8.8 & - & - & 1.6 & 0.95 & 1.1 & 1.0 \\
\hline 0307 & -3.5 & 14 & - & - & 3.3 & 1.2 & 1.4 & 0.99 \\
\hline $03 \cup 8$ & 35 & 11 & - & - & 4.1 & 1.1 & -1.6 & 1.0 \\
\hline & 35 & 9.4 & - & - & 3.2 & 1.0 & 1.3 & 0.79 \\
\hline 0309 & 11 & 13 & - & - & 4.2 & 1.3 & 0.82 & 0.87 \\
\hline $04 \cup 1$ & 18 & 13 & - & - & 1.0 & 1.3 & 0.0 & 0.91 \\
\hline 04U2 & 85 & 14 & - & - & 4.4 & 1.8 & -0.61 & 1.0 \\
\hline $04 U 3$ & 46 & 10 & 0.30 & 0.21 & 5.1 & 1.1 & 0.0 & 1.2 \\
\hline & 41 & 14 & - & 7 & 2.5 & 1.7 & -1.2 & 1.1 \\
\hline & 32 & 11 & - & - & 2.1 & 1.1 & 0.54 & 0.87 \\
\hline & 58 & 12 & - & - & 2.3 & 1.2 & -0.90 & 0.83 \\
\hline & 70 & 13 & - & - & 3.3 & 1.1 & -0.18 & 1.1 \\
\hline $04 U 4$ & 49 & 12 & - & - & 1.6 & 1.4 & -0.61 & 0.94 \\
\hline $04 U 5$ & 61 & 12 & - & - & 3.4 & 1.1 & 0.36 & 0.95 \\
\hline 0446 & 148 & 4.9 & 12 & 0.47 & 5.4 & 0.45 & 1.1 & 0.27 \\
\hline & 114 & 16 & - & 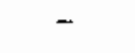 & 5.6 & 1.3 & 0.90 & 1.0 \\
\hline 0447 & 56 & 12 & - & - & 0.0 & 0.97 & 0.90 & 1.0 \\
\hline $04 \mathrm{U} 8$ & 31 & 10 & - & - & 0.41 & 1.1 & 0.36 & 0.90 \\
\hline 0409 & 18 & 11 & - & 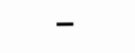 & 3.7 & 1.1 & -0.54 & 1.0 \\
\hline $05 \cup 3$ & -6.2 & 7.1 & - & - & -0.55 & 0.65 & -0.90 & 0.79 \\
\hline 0545 & 16 & 13 & - & - & 2.5 & 1. & 0.82 & 1.1 \\
\hline $05 \cup 6$ & 23 & 11 & - & 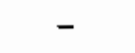 & 2.9 & 0.94 & 0.91 & 0.79 \\
\hline $05 U 7$ & 42 & 15 & 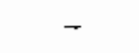 & . & 5.2 & 1.7 & 0.20 & 0.90 \\
\hline 0508 & 37 & 10 & - & _ & 0.41 & 1.0 & -0.54 & 0.74 \\
\hline
\end{tabular}


TABLE E.2.: (contd)

Variaoility Study no. 2

\begin{tabular}{|c|c|c|c|c|c|c|c|c|}
\hline \multirow{2}{*}{$\begin{array}{l}\text { Sample } \\
\text { Number }\end{array}$} & \multicolumn{2}{|c|}{ Thorium } & \multicolumn{2}{|c|}{ Radium } & \multicolumn{2}{|c|}{ Lead } & \multicolumn{2}{|c|}{ Uranium } \\
\hline & Level & Error & [eve? & Error & Eeve] & Error & Level & Error \\
\hline $\begin{array}{l}05 \text { U } \\
0649 \\
0301\end{array}$ & $\begin{array}{r}159 \\
28 \\
66\end{array}$ & $\begin{array}{c}22 \\
14 \\
9.5\end{array}$ & $\begin{array}{c}- \\
- \\
6.6\end{array}$ & $\begin{array}{l}- \\
- \\
1.3\end{array}$ & $\begin{array}{c}5.4 \\
-0.84 \\
3.4\end{array}$ & $\begin{array}{l}1.9 \\
1.6 \\
0.85\end{array}$ & $\begin{array}{l}1.8 \\
4.0 \\
1.1\end{array}$ & $\begin{array}{l}1.1 \\
1.8 \\
0.70\end{array}$ \\
\hline $\begin{array}{l}0302 \\
0401 \\
0402 \\
0502\end{array}$ & $\begin{array}{c}167 \\
51 \\
88 \\
3.5\end{array}$ & $\begin{array}{c}18 \\
12 \\
13 \\
7.0\end{array}$ & $\begin{array}{l}- \\
4.9 \\
7.0 \\
1.5\end{array}$ & $\begin{array}{l}- \\
1.4 \\
1.5 \\
1.1\end{array}$ & $\begin{array}{l}7.0 \\
3.2 \\
2.3 \\
1.1\end{array}$ & $\begin{array}{l}1.6 \\
1.0 \\
1.3 \\
1.0\end{array}$ & $\begin{array}{c}1.3 \\
0.6 \\
0.30 \\
-0.81\end{array}$ & $\begin{array}{l}1.1 \\
0.72 \\
0.75 \\
0.61\end{array}$ \\
\hline
\end{tabular}




\section{APPENDIX $F$}

PROPOSED REVISED RIO PUERCO CLEANUP CRITERIA 


\title{
APPENDIX F
}

\section{PROPOSED REVISED RIO PUERCO CLEANUP CRITERIA}

\begin{abstract}
"The New Mexico Environmental Improvement Division (NMEID) on August 13, 1979 issued an Order to United Nuclear Corporation (UNC) which required UNC to comply with recommendations for a cleanup program as described in a letter from Mr. Thomas E. Baca, NMEID, to Mr. D. D. Turberville, UNC, of the same date. This letter refers to condition seven of that August 13 letter.

"In accordance with Section 4-100B of the New Mexico Regulations for Governing the Health and Environmental Aspects of Radiation, every reasonable effort should be made to maintain radiation exposure as far below the limits specified in Part Four as practicable. Pursuant to this section, the staff of the Environmental Improvement Division has evaluated cleanup reports submitted by the United Nuclear Corporation, and performed an assessment of the radiation exposures resulting from the increased radionuclide soil concentrations in the areas affected by the spill. Based on the results of these evaluations, the interim cleanup criteria given in condition number seven of the August 13, 1979 letter will be replaced by the following:
\end{abstract}

7A. All contaminated areas shall be cleaned up to background levels of all radionuclides. This criteria shall be considered satisfied if the mean concentrations of thorium-230, radium-226 and lead-210, averaged over nine square feet, are less than or equal to

$$
\begin{array}{ll}
\text { thorium-230 } & 3 \mathrm{pCi} / \mathrm{gram} \\
\text { radium-226 } & 3 \mathrm{pCi} / \mathrm{gram} \\
\text { lead-210 } & 3 \mathrm{pCi} / \mathrm{gram}
\end{array}
$$

at the 50 confidence level.

7B. Those areas for which it has not been possible to meet the criteria given in Part 7A, upon approval by the Division, shall comply with the following criteria:

i. Mean concentrations of thorium-230, radium-226, and lead-210 shall not exceed the following limits:

$$
\begin{array}{ll}
\text { thor ium-230 } & 60 \mathrm{pCi} / \mathrm{gram} \\
\text { radium-226 } & 10 \mathrm{pCi} / \mathrm{gram} \\
\text { lead-210 } & 10 \mathrm{pCi} / \mathrm{gram}
\end{array}
$$

For the purpose of compliance with this criteria radionuclide concentrations may be averaged over areas with length of no more than $1000 \mathrm{ft}$ parallel to the arroyo, and width defined by the bases of the cut banks of the arroyo. This width shall not exceed $100 \mathrm{ft}$. Those arroyo areas whose 
wioths exceed $100 \mathrm{ft}$ must be separated into $1000-\mathrm{ft}$ by 100-ft segments, and the railonuclide concentrations averaged over each segment. All samples to be included in these averages must be taken from the area between the bases of the cut banks.

ii. Mean concentrations of thorium-230, radium-226, and lead-210 when averaged over nine square feet, shall not exceed the following limits:

$\begin{array}{lr}\text { thorium-230 } & 150 \mathrm{pCi} / \mathrm{gram} \\ \text { radium-226 } & 10 \mathrm{pCi} / \mathrm{gram} \\ \text { lead-210 } & 20 \mathrm{pCi} / \mathrm{gram}\end{array}$

iij. The limits given in Parts 7B.i. and 7B.ii above will apply at the $67 \%$ upper confidence level. So $i$ samples shal1 be taken in accordance with the standardized soil sampling technique described in the September 25, 1979, letter from Or. Ted Wolff, NMEID, to Mr. John AbDiss, UNC." 
NUREG-CR-2449

PNL. -4122

RW

\section{DISTRIBUTION}

No. of

Copies

OFFSITE

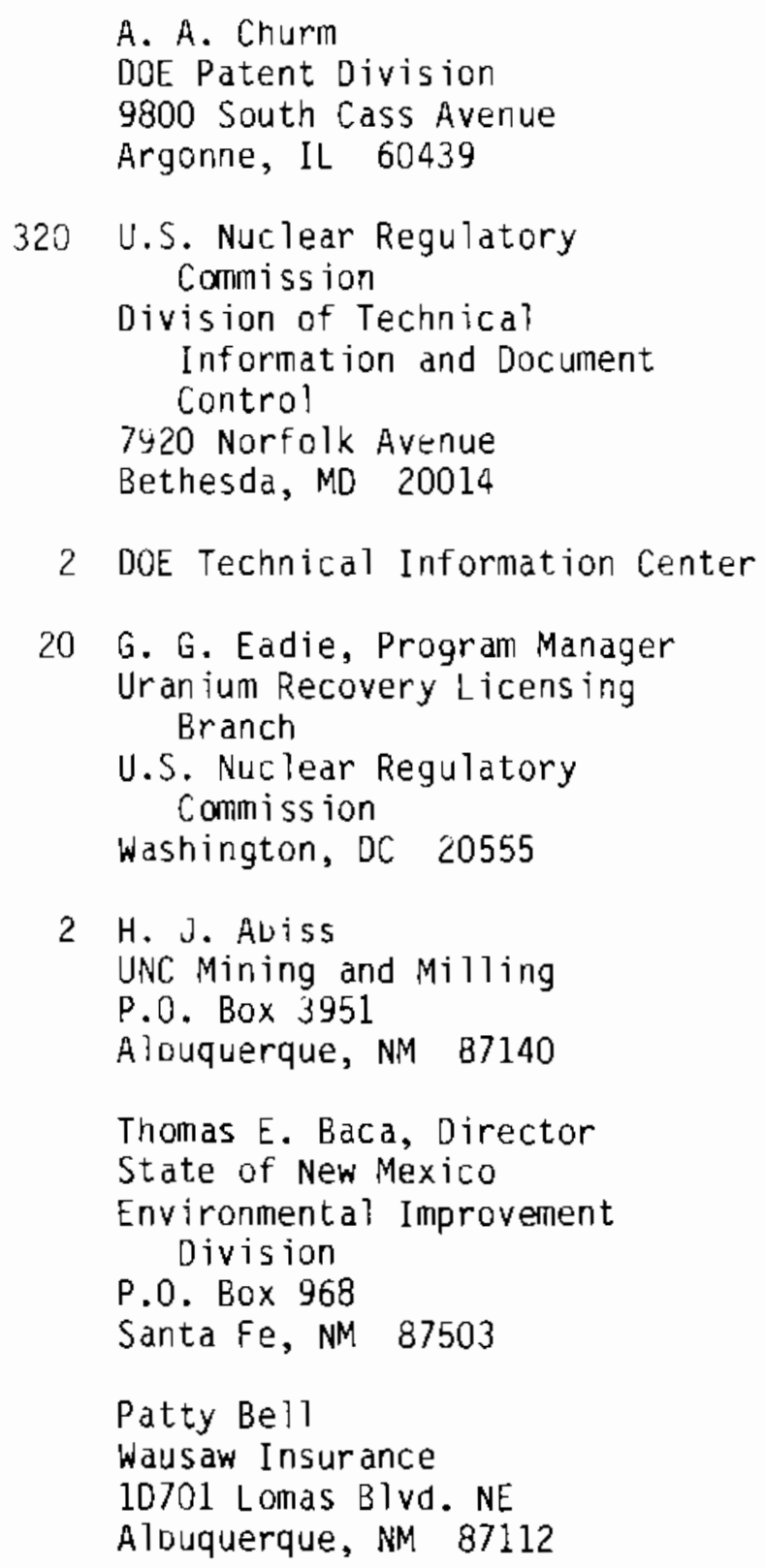

A. A. Churm

DOE Patent Division

9800 South Cass Avenue

Argonne, IL 60439

320 U.S. Nuclear Regulatory Commission

Division of Technical

Information and Document

Control

7920 Norfolk Avenue

Bethesda, MD 20014

2 DOE Technical Information Center

20 G. G. Eadie, Program Manager

Uran ium Recovery Licensing Branch

U.S. Nuclear Regulatory

Commission

Washington, DC 20555

2 H. J. Auiss

UNC Mining and Milling

P.0. Box 3951

A touquerque, NM 87140

Thomas E. Baca, Director

State of New Mexico

Environmenta? Improvenent Division

P.0. Box 968

Santa Fe, NM 87503

Patty Bel1

Wausaw Insurance

10701 Lomas Blvd. NE

Alduquerque, NM 87112

No. of

Copjes

D. Bernhardt

Office of Radiation Programs

Environmental Protection Agency

ORP/LVF

P.0. 80x 18416

Las Vegas, NV 89114

W. Bliss

U.S. EPA

ORP-LVF

P.0. Box 18416

Las Vegas, NV 89114

Glen Brown

U.S. NRC, Region IV

611 Ryan Plaza Drive

Arlington, $T X 76011$

R. Cooperste in

Environmental and Safety

Engineering Division

U.S. Department of Energy

Washington, DC 20545

Henry Folk, M.D.

Chronic Diseases Division

Bureau of Epidemiology

Center for Disease Control

At lanta, GA 30333

Helen George

Church Rock Action Committee

The Navajo Nation

Window Rock, AZ 86515

J. R. Giedt

Regional Radiation Representative Environmental Protection Agency

1860 Lincoln Street

Denver, Co 80295 
No. of

Copies

D. H. Groe 7 sema

U.S. Department of Energy

Washington, DC 20545

D. F. Harmon

U.S. Nuclear Regulatory

Commission

Washington, DC 20555

Fred Haywood

Health and Safety Research

Division

Oak Ridge National Laboratory

P.0. Box $x$

Oak Ridge, TN 37830

A. J. Hazle, Director

Radiation and Hazardous Waste Control Division

Colorado State Department of Health

1405 Curtis Street

Denver, CO 80202

G. Wayne Kerr, Director

Office of State Program

U.S. NRC

Washington, DC 20555

W. Kisieliski

Argonne National Laboratory

9700 South Cass Avenue

Argonne, IL 60439

Kathleen Kreiss, M.D.

Center for Disease Control

Atlanta, GA 30333

Glen Lane

Council of Energy Resource

Tribes

Technical Service Center

5660 South Syracuse Circle

Suite 206

Englewood, c0 80111
No. of

Copies

Peter MacDonald, Chairman

Navajo Tribal Council

Window Rock, AZ 86515

Henry May

U.S. EPA, Region VI

1201 Elm Street

Dallas, TX 75270

10 Jere B. Millard

State of New Mexico

Environmental Improvement

Division

P.0. Box 968

Santa Fe, NM 87503

2 Todd Miller

United Nuclear Corporation

Church Rock Uranium Mill

Gallup, NM 87301

M. Momeni

Argonne National Laboratory

9700 South Cass Avenue

Argonne, IL 60439

Office of Director

U.S. EPA

Office of Radiation Program

Washington, DC 20460

H. Rarrick

Sandia Area Office

U.S. Department of Energy

P.0. Box 5400

Aibuquerque, NM 87115

Or. Chet Sautter

P.0. Box 1858

Window Rock, AZ 86515

R. A. Scarano

U.S. Nuclear Regulatory Commission

Washington, DC 20555 
No. of

Copies

D. Sly

Office of Inspection \&

Enfor cement

U.S. NRC

Washington, DC 20555

Lor i Street

Edlow Internationa?

110017 th Street, Nw

Suite 404

Washington, DC 20036

T. L. Stumph

U.S. EPA, Region IX

215 Fremont Street

San Francisco, CA 94105

Glen Switchs

4152 Howe Street

0akland, CA g4611

Jim Szecsody

Desert Research Institute

Water Resources Center

P.0. Box 60220

Reno, NV 89506

2 Harold Tso

The Navajo Nation (NEPC)

Window Rock, AZ 86575

J. Thrall

Office of Radiation Programs

Environmental Protection Agency ORP/LVF

P.0. Box 18416

Las Vegas, NV 89114
No. of

Copies

J. Tipton

EG\&G, Incorporated

P.0. Box 1912

Mail Stop P-01

Las Vegas, NV 89101

J. Witherspoon

Oak Ridge National Laboratory

P.0. Box $X$

Oak Ridge, TN 37830

J. Womack

Indian Health Service

P.0. Box G

Window Rock, AZ 86515

R. Wynveen

Occupational Health and Safety Division

Argonne National Laboratory

9700 South Cass Avenue

Argonne, IL 60439

ONSITE

27 Pacific Northwest Laboratory

R. R. Kinnison

J. M. Nielsen

R. W. Perkins (5)

J. H. Reeves

L. C. Schwendiman

W. C. Weimer (10)

N. A. Wogman

Technical Information (5)

Publishing Coordination (2)(KC) 


\begin{tabular}{|c|c|c|}
\hline \multirow{3}{*}{ 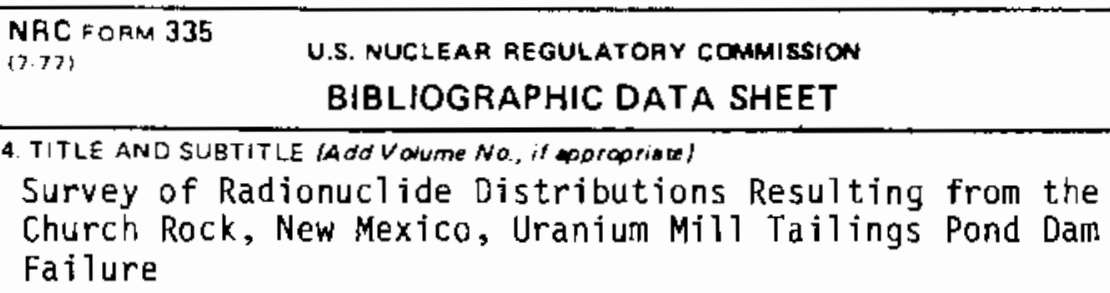 } & \multicolumn{2}{|c|}{$\begin{array}{l}\text { 1. REPOAT NUMBER Assigned by DOCI } \\
\text { NUREG/CR-2449 } \\
\text { PNL }-4122\end{array}$} \\
\hline & \multicolumn{2}{|l|}{ 2. (Leave bien $k$ ) } \\
\hline & \multicolumn{2}{|c|}{ 3. RECIPIENT'S ACCESSION NO. } \\
\hline \multirow{2}{*}{$\begin{array}{l}\text { 7. AUTHOR(S\} } \\
\text { W. C. Weimer, R. R. Kinnison, J. H. Reeves }\end{array}$} & \\
\hline & $\begin{array}{l}\text { MONTH } \\
\text { November }\end{array}$ & $\begin{array}{l}\text { TYEAR } \\
1981\end{array}$ \\
\hline 9. PERFORMING ORGANIZATION NAME AND MAILING ADORESS /InElude $Z_{i o}$ Code) & \multicolumn{2}{|c|}{ DATE REPORT ISSUED } \\
\hline \multirow{2}{*}{$\begin{array}{l}\text { Pacific Northwest Laboratory } \\
\text { Richland, Hashington } 99352\end{array}$} & \multicolumn{2}{|c|}{\begin{tabular}{l|r} 
MONTH & YEAR \\
December & $198]$ \\
\end{tabular}} \\
\hline & \multicolumn{2}{|c|}{ 6. (Leave bisnk) } \\
\hline & \multicolumn{2}{|l|}{ 8. (Leave biank) } \\
\hline \multirow{2}{*}{$\begin{array}{l}\text { 12. SPONSORING ORGANIZATION NAME AND MAILING ADDAESS anchude Zip Codef } \\
\text { U. S. Nuclear Regulatory Commission } \\
\text { Office of Nuclear Material Safety and Safeguards } \\
\text { Division of Waste Management } \\
\text { Washington, D.C. } 20555\end{array}$} & \multicolumn{2}{|c|}{ 10. PAOJE CT/TASKIWORK UNIT NO. } \\
\hline & \multicolumn{2}{|c|}{$\begin{array}{l}\text { 11. CONTRACT NO. } \\
\text { FIN No. B-2214 }\end{array}$} \\
\hline \multicolumn{3}{|c|}{\begin{tabular}{|l|l|} 
13. TYPE OF REPORT & PE RIOD COVERED (inciusive dares) \\
Final &
\end{tabular}} \\
\hline $\begin{array}{l}\text { 15. SUPPLEMENTARY NOTES } \\
\text { None }\end{array}$ & \multicolumn{2}{|l|}{ 14. (Leave biank) } \\
\hline \multicolumn{3}{|c|}{$\begin{array}{l}\text { In response to the uranium mill tailings pond dam failure on July } 16,1979 \text { at church } \\
\text { Rock, New Mexico, the U. S. Nuclear Reguiatory Commission through a contract with the } \\
\text { Pacific Northwest Laboratory provided an on-site mobile laboratory capable of } \\
\text { completing rapid radionuclide analyses of soil samples. This effort was necessary to } \\
\text { evaluate the extent of contamination caused by the accident, and to aid the mill } \\
\text { operator in its cleanup operations. The soi] sampling program and the review of the } \\
\text { operator's cleanup efforts in the affected areas was directed by the State of } \\
\text { New Mexico Environmental Improvement Division. This report discusses the results of } \\
\text { the radionuclide analyses of about } 1200 \text { soil samples taken from September } 22 \text {, } 1979 \text { to } \\
\text { December } 13 \text {, } 1979 \text {. These results indicate that the concentrations of uranium, } \\
\text { radium-226, and lead-210 were not distinguishable from natural background concentrations } \\
\text { However, certain areas had levels of thorium-230 greater than background concentrations, } \\
\text { and the operator concentrated cleanup efforts in these areas. As a result of this } \\
\text { program, it was estimated that the tota? inventory of thorium-230 released from this } \\
\text { accident was about } 31.4 \text { Curies above background. Sediment samples from the Grand Canyon } \\
\text { National Park showed no radionuclide levels in excess of normal backaround. }\end{array}$} \\
\hline \multicolumn{3}{|c|}{ 17. KEY WORDS AND DOCUMENT ANALYSIS 17a. DESCRIPTORS } \\
\hline \multicolumn{3}{|l|}{ 17b. IDENTIFIERS/OPEN.ENDED TERMS } \\
\hline \multirow{2}{*}{$\begin{array}{l}18 \text { AVAILABILITY STATEMENT } \\
\text { Unlimited }\end{array}$} & $\begin{array}{l}\text { Y CLASS (Thrs report) } \\
\text { fied }\end{array}$ & 21. NO OF PAGES \\
\hline & $\begin{array}{l}\text { YcLASS } \text { (This page } t \\
\text { fied }\end{array}$ & 22 PRICE \\
\hline
\end{tabular}


